\title{
Airborne Gravity Across New Zealand - For An Improved Vertical Datum
}

\author{
Jack McCubbine
}

supervised by

Dr. Euan Smith \& Dr. Fabio Caratori Tontini \& Dr. Matt Amos

\section{A thesis}

submitted to the Victoria University of Wellington in fulfilment of the requirements for the degree of Doctor of Philosophy 


\begin{abstract}
It is important to be able to accurately determine the height of a point on the Earth in terms of the Earth's gravitational potential field. These heights predict how water will flow and so they are vital for engineering and surveying purposes. They are determined using a vertical datum which consists of a specified height system and a defined reference surface.
\end{abstract}

At present, in New Zealand, the official vertical datum is NZVD2009 which uses a normal-orthometric height system and gravimetric quasigeoid, NZGeoid2009, as the reference surface. The aim of this thesis is to develop a more accurate gravimetric quasigeoid than NZGeoid2009, by incorporating new gravity data and utilising a refined data processing strategy, to establish a better vertical datum for New Zealand.

A new airborne gravimetry data set has been collected which covers the North, South and Stewart Islands of New Zealand with a flight line spacing of $10 \mathrm{~km}$. The data were susceptible to short error prone sections of track due to poor (turbulent) flight conditions and mean offsets which separate the recorded gravity data along flight lines by a constant value from neighbouring lines and existing gravity models. The error prone sections of track have been visually identified by assessing the cross track agreement with other flight lines and with the global gravity model EGM2008, and the mean offsets were estimated by a least squares method which takes into consideration the spatially correlated gravity signal.

The repeatability of the data was assessed from data collected from five flights along two separate calibration lines. The mean gravity anomaly profiles calculated along the calibration lines each had a standard deviation of around $2.5 \mathrm{mGal}$. The internal consistency of the data was assessed by evaluating the difference between flight line data at intersection points. This accuracy measure was shown to be influenced by the along track filter, anisotropic topography and the relative flight line elevations. After correcting for all these effects the set of all intersecting differences had a standard deviation of approximately $5.9 \mathrm{mGal}$.

From an existing terrestrial gravity database, around 40000 observations have been reprocessed to reduce them to Bouguer gravity anomalies, this was done to ensure consistency in the formulas that have been used. A new national $8 \mathrm{~m}$ digital elevation model (DEM) was used to calculate terrain corrections and these were carefully compared with terrain corrections estimated from field observations of the topography to reduce any discrepancies in calculating near zone terrain effects. The largest source of error in the terrestrial gravity anomaly data is due to inaccurate height estimates of the marks. The height discrepancies have been estimated by comparing the recorded heights in the database to those determined from the $8 \mathrm{~m}$ DEM and have been translated into $\mathrm{mGal}$ by calculating the propagated effect on the free air and Bouguer slab corrections. 
The airborne and terrestrial gravity data, along with a satellite altimetry marine gravity anomaly and existing shipborne gravity data, were assimilated by least squares collocation with a logarithmic covariance function to appropriately deal with the downward continuation of the airborne data, and gridded at 1 arc-minute resolution in the geographical region $25^{\circ}(\mathrm{S})$ to $60^{\circ}(\mathrm{S})$ and $160^{\circ}(\mathrm{E})$ to $190^{\circ}(\mathrm{E}) .1$ arc-minute block averaged heights were then used to calculate a reverse Bouguer slab correction, which when applied to the gravity data gave a gridded Faye anomaly. Different noise level variances were assigned to the separate data sets to optimally combine them.

Forty six of the most contemporary global gravity models (from 2008 onwards) have each been compared to 1422 leveling and GNSS derived quasigeoid height anomalies. Overall the Eigen-6C4 model fitted the leveling and GNSS derived quasigeoid height anomalies best with a root mean squared error of $5.29 \mathrm{~cm}$.

The Eigen-6C4 gravity model was subtracted from the gridded Faye anomaly (remove) and Stokes integral was evaluated on the residual gravity anomaly grid. A, theoretically optimum, modified Stokes kernel has been used and the modification degree $L$ and spherical cap for the integration $\psi_{0}$ were varied over the ranges $L=20,40,60, \ldots, 320$ and $\psi_{0}=1^{\circ}, 1.5^{\circ}, 2^{\circ}, 2.5^{\circ}, 3^{\circ}$. The Eigen-6C4 geoid undulations were then added back to the residual geoid undulation grids and the primary indirect topographic effect was restored to obtain 80 quasigeoids for each $L$ and $\psi_{0}$ parameter variation.

The optimal parameter choice was determined to be $L=280$ and $\psi_{0}=1.5$ which had the best agreement with the leveling and GNSS derived quasigeoid height anomalies with a standard deviation of $3.8 \mathrm{~cm}$ and root mean squared residual of $4.8 \mathrm{~cm}$ of the differences. This is a $1.25 \mathrm{~cm}$ improvement on NZGeoid2009. The quasigeoid was also assessed closely in three main urban areas, Auckland, Wellington and Christchurch, where the majority of large scale engineering projects and surveying takes place in New Zealand. Here there were 123, 169 and 125 data points and the standard deviations of the differences were $3.976,3.385$ and $2.071 \mathrm{~cm}$ and root mean squared differences of $3.58,4.388$ and $4.572 \mathrm{~cm}$ respectively. This gives an average accuracy of $3.1 \mathrm{~cm}$ standard deviation in urban areas which is $1.5 \mathrm{~cm}$ better than the average for NZGeoid2009. 


\section{Contents}

Introduction and Thesis Overview $\quad 5$

$\begin{array}{ll}\text { Chapter synopsis } & 11\end{array}$

$\begin{array}{ll}\text { Glossary } & 14\end{array}$

1 Heights and Reference Surfaces $\quad 16$

1.1 Introduction . . . . . . . . . . . . . . . . . . . . 16

1.2 Height reference surfaces . . . . . . . . . . . . . . . . . . 16

1.3 Height systems . . . . . . . . . . . . . . . . . . . . . . . 18

1.3.1 Geometric height systems . . . . . . . . . . . . . 18

1.3.2 Physical height systems _. . . . . . . . . . . . . . . . 19

1.4 Summary . . . . . . . . . . . . . . . . . . . . . . 24

2 The Disturbing Potential, Gravity Anomalies and Geoid Heights 25

2.1 Introduction . . . . . . . . . . . . . . . . 25

2.2 The disturbing potential and gravity anomalies . . . . . . . . 26

2.2.1 Normal gravity . . . . . . . . . . . . . . . . . . . 27

2.2 .2 Free air effect . . . . . . . . . . . . . . . . . 27

2.2.3 Bouguer slab and terrain corrections . . . . . . . . . . . 28

2.3 Upward and downward continuation of gravity anomalies . . . . . . . . . 35

2.4 Earth tides . . . . . . . . . . . . . . . . . . . . 42

2.5 Geoid undulations determined by Stokes' integral . . . . . . . . . . . . 43

2.5.1 Helmert's second method of condensation: . . . . . . . . . . . . 44

2.5.2 Remove - Compute - Restore (RCR) . . . . . . . . . . . . . 45

2.5.3 Stokes' kernel modification: . . . . . . . . . . . . . . 45

2.6 Summary . . . . . . . . . . . . . . . . . . . . . . . 48

3 Vertical Datums in New Zealand $\quad 49$

3.1 What is a vertical datum? . . . . . . . . . . . . . . . . . . 49

3.2 New Zealand's Local Vertical Datums . . . . . . . . . . . . . . . . . . . . 49

3.3 NZGeoid2005: local vertical datum unification . . . . . . . . . . . 52

3.4 NZVD2009 . . . . . . . . . . . . . . . . . . . 53

3.5 Summary . . . . . . . . . . . . . . . . . . 60 
4 Terrestrial Gravity in New Zealand $\quad 62$

4.1 Introduction . . . . . . . . . . . . . . . . . . . . 62

4.2 GNS Science terrestrial gravity database . . . . . . . . . . . . . . . . 62

4.3 Gravity reduction . . . . . . . . . . . . . . . . 65

4.4 Terrain Corrections . . . . . . . . . . . . . . . . . . 67

4.5 Accuracy assessment . . . . . . . . . . . . . . . . . . 71

4.6 Summary . . . . . . . . . . . . . . . . . . . 77

5 Global Gravity Models (GGM) $\quad 78$

5.1 Introduction . . . . . . . . . . . . . . . . . . . . . 78

5.2 Spherical harmonic coefficients . . . . . . . . . . . . . . 78

5.3 GGM comparison . . . . . . . . . . . . . . . . . . 80

5.3.1 Satellite only global gravity models: . . . . . . . . . . . . . . . 81

5.3.2 Combined global gravity models. . . . . . . . . . . . . . . 84

5.4 Summary . . . . . . . . . . . . . . . . . . . . . . 87

6 Airborne Gravity across New Zealand 89

6.1 Introduction . . . . . . . . . . . . . . . . . . 89

6.1.1 What is Airborne Gravimetry? . . . . . . . . . . . . . . . 89

6.2 The New Zealand national airborne gravity survey . . . . . . . . . . . . . 91

6.2.1 The Aircraft . . . . . . . . . . . . . . . . . . . . . 93

6.2.2 The GNSS and post processing methods . . . . . . . . . . . . 93

6.2 .3 The gravimeter . . . . . . . . . . . . . . . . . . 95

6.3 Gravity reductions . . . . . . . . . . . . . . . . . . 97

6.3.1 Vertical Accelerations and Synchronising the GPS with the Gravimeter measurements . . . . . . . . . . . . . . . . 99

6.3 .2 Tilt Correction . . . . . . . . . . . . . . . . . 101

6.3.3 The Eotvos effect . . . . . . . . . . . . . . . . . . . . . . . 102

6.3.4 The Free Air gravity anomaly . . . . . . . . . . . . . . . . . 103

6.3.5 Low Pass Filtering . . . . . . . . . . . . . . . . . 105

6.3.6 Terrain corrections for the airborne gravity data . . . . . . . . . 106

6.4 Detecting erroneous sections of track . . . . . . . . . . . . 108

6.5 Flight line biases . . . . . . . . . . . . . . . . . . . . . . . . . . . 112

6.6 Accuracy assessment . . . . . . . . . . . . . . . . 127

6.6.1 Cross-over error . . . . . . . . . . . . . . . . . . . 127

6.6.2 Comparison to the terrestrial gravity data. . . . . . . . . . . . . 140

6.6.3 Repeatability of the airborne gravity data - the calibration lines . 145

6.6.4 Summary of accuracy assessment . . . . . . . . . . . . . . . . 148

6.7 Summary . . . . . . . . . . . . . . . . . . . 150

7 Combining Data Sets using Least Squares Collocation (LSC) 151

7.1 Introduction . . . . . . . . . . . . . . . . . . . . . . . 151

7.2 Basic mathematical setting . . . . . . . . . . . . . . . . 152

7.3 Covariance functions . . . . . . . . . . . . . . . . . . . . 153

7.4 General steps taken to grid gravity anomalies using least squares collocation. 154

7.5 Combining all of the available datasets into a single gravity anomaly grid 155 
7.6 Summary . . . . . . . . . . . . . . . . . . . 178

8 Computation of a New Gravimetric Quasigeoid $\quad 180$

8.1 Introduction . . . . . . . . . . . . . . . . . . . . . 180

8.2 The gridded gravity anomaly used to determine a new quasigeoid . . . . 181

8.3 Remove - Compute - Restore . . . . . . . . . . . . . . . . . . . 184

8.3.1 Recap on the methodology of regional quasigeoid computation . . 184

8.3.2 Computational steps taken to compute the new gravimetric quasigeoid . . . . . . . . . . . . . . . . . . 185

8.4 Results . . . . . . . . . . . . . . . . . . . . . 188

8.4.1 Additional leveling/GPS points . . . . . . . . . . . . 193

8.4 .2 Summary of results . . . . . . . . . . . . . . . . . 197

8.5 Summary . . . . . . . . . . . . . . . . . . . . 198

9 Summary, Research Outcomes and Recommendations for Future Work200

9.1 Summary .......................... 200

9.2 Research Outcomes . . . . . . . . . . . . . . . . . . . . . 203

9.3 Future Work . . . . . . . . . . . . . . . . . . . . . . . . 204

$\begin{array}{ll}\text { References } & 206\end{array}$

$\begin{array}{ll}\text { Appendices } & 213\end{array}$

A Calculating the Lacoste and Romberg model 'S80' gravimeter accelerometer MV to $\mathrm{mGal}$ scale factor $\quad \mathbf{2 1 3}$

A.1 Introduction . . . . . . . . . . . . . . . . . . . . 213

A.2 Methodology . . . . . . . . . . . . . . . . . 213

A.3 Results . . . . . . . . . . . . . . . . . . . . . 215

B Fitting Gaussian distributions to leptokurtotic data sets 217

B.1 Standard methodology . . . . . . . . . . . . . . . . . 217

B.2 Derivation of an alternative method to derive the standard deviation of an empirical distribution in the presence of outliers. . . . . . . . . . . . . . 219

B.2.1 Advantages of this method . . . . . . . . . . . . . . 220

B.3 A worked example using data from section 4 . . . . . . . . . . . . . 222

$\begin{array}{ll}\text { C Electronic Appendices } & 224\end{array}$

$\begin{array}{ll}\text { List of figures } & 226\end{array}$

$\begin{array}{ll}\text { List of tables } & 233\end{array}$ 


\section{Acknowledgments}

I would like to express my special appreciation and thanks to my three supervisors, Dr. Euan Smith, Dr. Fabio Caratori Tontini and Dr. Matt Amos. You have each been tremendous mentors and I would like to thank you for inspiring and encouraging me. Your advice and guidance throughout the project have been extremely valuable. I would also like to thank Land Information New Zealand for funding the research and GNS Science for providing me with a space to work and all the facilities I have needed. I also want to thank Rachelle Winefield for her unwavering help and stimulating conversation throughout the project.

I would also like to acknowledge the guidance offered by both Dr. René Forsberg and Dr. Theresa Damiani with the airborne gravity data processing, and Dr. Vaughan Stagpoole for sharing his knowledge of the New Zealand terrestrial gravity data. Your help has been instrumental in the successful completion of this project.

A special thanks to my family. Words cannot express how grateful I am to my mum and dad, Gill and Duncan, for all of the sacrifices that they have made on my behalf and support throughout my studies. I would also like to thank my brother, Tom, and sister, Charlotte, for providing comic relief and helping me keep things in perspective. Finally I would like to thank my partner Phillipa for her endless patience and calming influence. 


\section{Introduction and thesis overview}

Background: It is vital to accurately know the height of a point on the surface of the Earth relative to mean sea level for large scale engineering projects and surveying. Mean sea level coincides with an equipotential surface in the Earth's gravity field and so heights defined above this surface are inherently related to the force of gravity. Water will flow from points higher above the mean sea level to those closer to it. This is important for engineering projects. For example, if a pipe is laid such that fluid is required to flow through the pipe under the influence of gravity alone, the end of the pipe water is flowing from must be higher above mean sea level than the end it is flowing to.

Heights above mean sea level on land have traditionally been obtained by leveling. This involves transferring mean sea level estimates at the coast (made using a tide gauge) inland using a leveling instrument (e.g. a spirit level) and a rod with a numbered scale. However obtaining heights in this manner is time consuming and expensive.

An approximation of the separation between mean sea level and an Earth approximating ellipsoid (hereafter referred to as the quasigeoid) can be obtained by a mathematical transformation of measured values of the force of gravity at a set of grid points covering inland areas. A quicker and more cost effective approach than leveling to obtain mean sea level heights inland is to obtain a height above an Earth approximating ellipsoid measured using a global navigation satellite system (GNSS) device and subtract the value interpolated from the gridded quasigeoid surface. Ellipsoidal heights determined using a GNSS device can be obtained to sub centimetre accuracy. The accuracy of heights above the quasigeoid calculated in this manner are heavily dependent on the quality of the gridded data.

The current official national quasigeoid for New Zealand is New Zealand Quasigeoid 2009 (NZGeoid2009). It was calculated by Stokes' integration, with the remove-computerestore technique, on a 1 arc-minute grid of gravity data (Claessens et al (2009)). The interpolated gravity data consisted of more than 40000 terrestrial gravity observations (from a GNS Science database, Stagpoole 2012) and the 2008 Danish National Space Centre satellite altimetry marine gravity anomaly (DNSC08). Earth gravity model 2008 (EGM2008) was used to provide the reference signal for the remove and restore stages of the calculation (Claessens et al, 2009). NZGeoid2009 is the reference surface for the current official vertical datum, New Zealand Vertical Datum 2009 (NZVD2009), which uses a normal-orthometric height system.

Until 2009, New Zealand had 13 official local vertical datums. There are discontinuities between the datums since they are each referenced to separate mean sea level estimations (from tide gauge measurements) which can differ by a constant. Each local vertical datum contains numerous leveling based height measurements and, as of 2009, 1422 of the leveling sites had had their ellipsoid height measured with a GNSS device. The ellipsoidal height minus the leveling derived heights give quasigeoid height anomalies. By grouping the data by one local vertical datum at a time a mean offset from the quasigeoid can be evaluated. This process unifies the otherwise separate datums and was first put to 
practice by Amos (2007).

The accuracy of NZGeoid2009 has been evaluated by comparing it to the GNSS and leveling height derived quasigeoid height anomalies after applying the mean local vertical datum offsets. The difference between the two data sets had a root mean squared residual of $6.144 \mathrm{~cm}$ and standard deviation (estimated from the inner $68 \%$ of the differences, see appendix B) of $4.8 \mathrm{~cm}$.

The terrestrial gravity data, used to calculate NZGeoid2009, largely consists of historic measurements, of varying accuracy, made by several different institutions using a variety of gravimeters. They are unevenly distributed, having an average spatial density of around $7 \mathrm{~km}$, and are particularly sparse in rough topography due to inaccessibility. Moreover, satellite altimetry data provide accurate mean sea level observations and gravity field observations over the open oceans. But it is unreliable in shallow coastal areas due to inaccurate tidal estimations and large sea surface variability (Hwang and Hsu, 2008). These qualities of the terrestrial and satellite altimetry gravity data are undesirable for geoid modelling but fortunately they can be overcome using airborne gravimetry.

Airborne gravimetry is a method of measuring the total accelerations experienced by an aircraft, which include both those due to gravity and those due to aircraft motion, and then removing the accelerations due to aircraft motion to estimate the acceleration due to gravity. The measurements are made using a highly accurate accelerometer. Several different types of these instruments exist, for example spring-based relative gravity meters which are mounted on a stabilized platform (Model 'S' Air and Sea Dynamic Gravity Meter Systems (2006), used by, Forsberg et al (2015,1999,2001), Olesen (2002)) which includes models such as the ZLS upgraded Lacoste and Romberg model 'S' gravimeters, Micro-g LaCoste (gravimeter models: TAGS, TAGS7) and Canadian Microgravity Ltd (model: GT-2A) etc., and strap down inertial measurement unit gravimeters which do not have a stabilized platform (Glennie et al (2000), Forsberg (2001b), Kwon (2001) etc.). Areas which would be inaccessible by land or sea (for example, through mountain ranges or shallow coastal areas) can be surveyed more easily when flying. This method can be used to overcome the shortcomings in the terrestrial and satellite altimetry gravity observations since the data can be collected on a uniform grid (i.e. with a regular spatial density), seamlessly covering on and offshore areas, using consistent instrumentation.

Airborne gravimetry has become more prevalent and reliable with the advent of accurate kinematic global positioning systems developed in the late 1980's. The airborne gravimeters measure the total vertical acceleration and so the positioning measurements make it possible to accurately model and remove specific forces associated with aircraft motion to obtain the residual gravity signal. Some of the first successful large scale airborne gravimetry surveys were conducted in Switzerland in 1992 (Klingele et al, 1996) and Greenland in 1991-1992 (Brozena, 1991). In Switzerland the whole country was surveyed at an altitude of around $5100 \mathrm{~m}$. The survey confirmed that accurate gravity data can be obtained over rugged topography which is plainly advantageous over traditional terrestrial measurements since these areas would be otherwise inaccessible. 
After downward continuing the measurements from the flight elevation, airborne gravimetry data can be used to model the geoid. Recent projects include a nationwide survey in Mongolia in 2004-2005 (Forsberg et al. 2007) which had the intention of providing data for EGM2008 as well as improving the local vertical datum, the survey in Taiwan in 2007 (Hwang et al, 2007) and the South Korean survey in 2008-2009 (Bae et al, 2012 and Yang, 2013).

In Mongolia the flight lines were spaced at 10 nautical miles flown at varying altitudes of $2100 \mathrm{~m}$ to $4800 \mathrm{~m}$. The data had an estimated accuracy of around $2.2 \mathrm{mGal}$ assessed by evaluating the agreement at flight line intersection points. The airborne data were downward continued and merged with existing terrestrial gravity measurements to develop a new geoid. The geoid had a reported accuracy of $20 \mathrm{~cm}$ on comparison to geoid height anomalies determined from GNSS/leveling data although it was argued that this value is not particularly representative of the true geoid accuracy due to datum offsets present in the data.

Taiwan's gravity field was reported to have extremely large variations between -400 to +400 mGal, due to extremely rugged topography. The existing terrestrial gravity measurement were extremely sparse due to the topography, so airborne gravity was used to obtain accurate short wavelength gravity data for geoid modelling. Here the flight line spacing was chosen to be $4.5 \mathrm{~km}$ and the average flight line altitude was around 5150 $\mathrm{m}$. After the removal of large outliers the estimated accuracy of the survey was around $2 \mathrm{mGal}$ from the flight line intersection discrepancies. The determined geoid was again assessed by comparison to GNSS/leveling data and determined to be centimetre accurate in coastal regions but only decimetre accurate in rougher topography.

In South Korea an airborne gravity survey was conducted with around 51 flight lines flown at around $3000 \mathrm{~m}$. The accuracy of the data was deduced by comparing the airborne measurements to terrestrial gravity data and had an agreement with a standard deviation of around $12 \mathrm{mGal}$. A gravimetric geoid determined from the data had an agreement with existing GNSS/leveling data of around $5 \mathrm{~cm}$.

The topography in Mongolia, Taiwan and South Korea can be particularly rugged and so similar to areas of New Zealand which have particularly sparse terrestrial gravity observations. The success of the airborne gravity surveys in assisting with accurate geoid determination in these cases indicates that it could be similarly effective in New Zealand.

Objective: An implicit assumption throughout this work is that the leveling and GNSS observations provide the best possible estimate of the quasigeoid height anomalies. Here an improved vertical datum is sought which is based on a gravimetric quasigeoid that is in better agreement with the leveling and GNSS derived quasigeoid height anomalies than NZGeoid2009. The fundamental step which must be taken to calculate a refined quasigeoid is to improve the underlying gravity data.

Steps taken to compute a more accurate quasigeoid: A new airborne gravimetry data set has been collected and carefully reduced to Bouguer anomaly measurements. 
The data cover the North, South and Stewart Islands of New Zealand with a flight line spacing of $10 \mathrm{~km}$. These data were susceptible to short erroneous sections of track due to poor flight conditions and mean offsets which separate the recorded gravity data along flight lines by a constant value from neighbouring lines and existing gravity models. The poor quality data have been removed by carefully assessing the cross track agreement with other flight lines and with the global gravity model EGM2008. Offsets in the flight lines have been estimated by a least squares method which takes into consideration the spatially correlated gravity signal, following Reilly (1979) and Forsberg (1987).

The repeatability of the airborne data was assessed by collecting repeat measurements along two separate calibration lines with 5 flights for each. The differences from a mean gravity anomaly calculated along the lines had a standard deviation of approximately $2.5 \mathrm{mGal}$. The internal consistency of the data was assessed by evaluating the difference between flight line data at intersection points. This accuracy measure is shown in chapter 6 to be susceptible to the along track filter, anisotropic topography and the relative flight line elevations. After correcting for all these effects the set of all intersecting differences had a standard deviation of approximately $5.9 \mathrm{mGal}$.

A new nation wide $8 \mathrm{~m}$ digital elevation mode (DEM) has become available since the computation of NZGeoid2009. These data were used to calculate new terrain corrections for the terrestrial gravity data. The DEM terrain corrections were carefully combined with terrain corrections that had been separately estimated from field observation topographic heights when the gravity observations were made. This was performed to reduce any discrepancies in calculating near zone terrain effects, using the DEM, for potentially mis-located observation sites. The $8 \mathrm{~m}$ DEM offers a significant improvement over the 56 $\mathrm{m}$ model used for terrain corrections for the NZGeoid2009 gravity anomaly data. The $8 \mathrm{~m}$ DEM was block averaged to a $64 \mathrm{~m}$ grid to ease the computational time when calculating terrain corrections for the airborne data since there were close to 1000000 measurements made during the survey. This results in a small loss in accuracy but it is negligible since the high frequency effect of the topography is greatly reduced at the altitude of the aircraft.

The largest source of error in the terrestrial gravity anomaly data arises from inaccurate height estimates which are needed for the free air and Bouguer slab corrections (Stagpoole, 2012). Heights have been estimated by either leveling and GNSS observations, read from map sheets or read from a barometer. These height measurement methods have estimated accuracies of $15 \mathrm{~cm}, 7.5 \mathrm{~m}$ and $9 \mathrm{~m}$ standard deviation respectively when comparing the recorded heights in the database to heights extracted from the $8 \mathrm{~m}$ DEM at the same points. These accuracy estimates have been translated into mGal by calculating the propagated effect on the free air and Bouguer slab corrections. The accuracy measures were assigned to each observation in the database and outliers were given appropriately large values.

The airborne and terrestrial gravity data, along with a satellite altimetry marine gravity anomaly and existing shipborne gravity data, were augmented by least squares collocation and gridded at 1 arc-minute resolution in the region $25(\mathrm{~S})$ to $60(\mathrm{~S})$ and $160(\mathrm{E})$ 
to 190(E). 1 arc-minute block averaged heights from the $8 \mathrm{~m}$ DEM were then used to calculate a reverse Bouguer slab correction, which when applied to the gravity data gives a gridded Faye anomaly (Claessen et all, 2009, Amos, 2007 and Featherstone and Kirby, 2000).

The Forsberg (1987) logarithmic covariance function was used for the least squares collocation, since it appropriately incorporates the gravity anomaly observations at varying elevations. Noise level variances were assigned to the terrestrial gravity data in accordance with the propagated gravitational effect of the height discrepancies to the gravity corrections, the airborne data were assigned a $9 \mathrm{mGal}^{2}$ variance in correspondence to the accuracy assessment, the shipborne gravity data were assigned a $4 \mathrm{mGal}^{2}$ variance and the satellite altimetry data variance estimates were taken from an estimated error supplied with the data.

On comparison to the gridded Faye anomaly data used to compute NZGeoid2009 it is shown by taking a difference between the two grids that there is a significant contribution from the airborne data in shallow coastal regions and through rough topography. This is because previously the data coverage had been sparse and unreliable. The airborne data have the effect of smoothing the transition from land to sea in the gridded data which results in a gravity anomaly grid which is seamless in coastal areas.

The most recent global gravity models (since 2008 onwards) available from http:/ /icgem.gfzpotsdam.de/ICGEM/modelstab.html (last accessed September 2015) have each been compared to the terrestrial gravity data and the 1422 leveling and GNSS derived quasigeoid height anomalies. Overall the Eigen-6C4 model fit the leveling and GNSS derived quasigeoid height anomalies best whilst the EGM2008 model fit the terrestrial gravity data best.

The terrestrial gravity data are not independent from the global gravity models and EGM2008 used the terrestrial data to determine a wider spectral range of spherical harmonic coefficients than Eigen-6C4 did; this is the reason it fits the gravity data better. Since the Eigen-6C4 gave the best fit to the leveling and GNSS quasigeoid height anomalies, which is an independent data set, it was determined to be the best choice as the reference signal for the remove and restore stages of a new quasigeoid computation.

The Eigen-6C4 gravity model was subtracted from the gridded Faye anomaly (remove) and Stokes integral was evaluated on the residual gravity anomaly grid. A Featherstone et al. (1998) modified Stokes kernel has been used and the modification degree $L$ and spherical cap for the integration $\psi_{0}$ were varied over the ranges $L=20,40,60, \ldots, 320$ and $\psi_{0}=1^{\circ}, 1.5^{\circ}, 2^{\circ}, 2.5^{\circ}, 3^{\circ}$. This resulted in 80 residual quasigeoid undulation grids. The computation took approximately 30 hours for each parameter variation and four instances of the program were run simultaneously.

The Eigen-6C4 geoid undulations were then added back to the residual geoid undulation grids and the primary indirect topographic effect was restored to obtain 80 quasigeoids for each $L$ and $\psi_{0}$ parameter variation. 
Results, the quasigeoid accuracy assessment: Each quasigeoid has been compared to the leveling and GNSS derived quasigeoid height anomalies. The optimal parameter choice was determined to be $L=280$ and $\psi_{0}=1.5$ which had the best agreement with the leveling and GNSS derived quasigeoid height anomalies with a standard deviation of $3.8 \mathrm{~cm}$ and root mean squared value of the differences of $4.8 \mathrm{~cm}$. This is a 1.25 cm improvement on NZGeoid2009.

The $L=280$ and $\psi_{0}=1.5$ quasigeoid and leveling and GNSS derived quasigeoid height anomalies agreement was also assessed closely in 3 main urban areas, Auckland, Wellington and Christchurch. Here there were 123, 169 and 125 data points respectively and the standard deviations were $3.976,3.385$ and $2.071 \mathrm{~cm}$ and root mean squared of $3.58,4.388$ and $4.572 \mathrm{~cm}$ respectively. This gives an average accuracy of $3.1 \mathrm{~cm}$ standard deviation in urban areas which is $1.5 \mathrm{~cm}$ better than the average for NZGeoid2009.

In summary, a quasigeoid has been computed in the region $25(\mathrm{~S})$ to $60(\mathrm{~S})$ and $160(\mathrm{E})$ to 190(E) at a 1 arc-minute resolution. The quasigeoid is based on new airborne gravity data, refined terrestrial and satellite altimetry gravity anomalies and, unlike NZGeoid2009, includes the existing shipborne data. Moreover a new and more accurate global gravity model, Eigein-6C4, has been used as the reference signal for the remove and restore stages of the quasigeoid computation. The improved gravity data and more accurate reference global gravity model has produced a quasigeoid with a better agreement with leveling and GNSS derived quasigeoid height anomalies than the current official national quasigeoid, NZGeoid2009. For this reason it would make a suitable replacement to establish a new national vertical datum.

Personal involvement in data acquisition I was personally involved in all stages of the airborne data acquisition and establishing ground based observation sites for the calibration line terrestrial gravity measurements.

I was trained how to operate the gravimeter used for the airborne survey which is an instrument owned by GNS Science. During the survey period I was on board at least $50 \%$ of the flights during which time I was solely responsible for operating the gravimeter and instrumentation (GNSS, navigation software for the flight path etc). The gravimeter requires regular observation even when it is not being used for airborne data collection to ensure it is functioning properly, I was additionally responsible for this during the survey period.

The terrestrial gravity observations along the calibration flight lines were made at regular intervals of around $500 \mathrm{~m}$ resulting in around 120 measurement sites. Each of these sites needed to be located using a GNSS and physically marked to ensure gravity measurements were made at precisely the same location. I was personally involved in establishing each of these sites, measuring their location using a GNSS device and physically marking the sites by driving posts/rods into the ground. 


\section{Chapter synopsis}

Chapter 1 : defines the concept of heights. The distinction between geometrical and physical height systems is given. The main height systems, ellipsoidal, orthometric (and approximations), normal and normal-orthometric heights, and the corresponding reference surfaces, the ellipsoid, geoid, telluroid and quasigeoid, are described in detail. The draw-backs and advantages of each height system are discussed.

Chapter 2 : defines the disturbing potential and gravity anomalies and how they are related mathematically. Formulas which approximate the gravitational effect of an Earthapproximating ellipsoid are given. It is discussed how the gravitational effect of the topography can be estimated from field observations and by using a digital elevation model. The effect of making a gravity observation at an elevation above a gravitational source is demonstrated and the methods to downward continue a gravity signal from one elevation to another are identified. The concept of the permanent tidal effects of the sun and moon is given and three key models are specified, the preferred choice for geoid modelling made by the International Association of Geodesy in 1993 is identified. Finally, the mathematical relationship between gravity anomalies and geoid undulations is shown and the main computational steps which need to be taken to compute a regional quasigeoid are specified.

Chapter 3 : describes the concept of a vertical datum. New Zealand's 13 separate vertical datums are described in detail and the first attempt to unify them using a gravimetric quasigoied, computed by Amos (2007), is discussed. The current official vertical datum NZVD2009 is identified and the computational steps taken to calculate the reference quasigeoid, NZGeoid2009, and the gravity data used to determine it are given. A brief accuracy assessment of NZGeoid2009 is performed by a comparison to leveling and GNSS derived quasigeoid height anomalies and the effect of the mean offsets of the local vertical datums is demonstrated.

Chapter 4 : describes the GNS Science terrestrial gravity database. An overview of the instrumentation used to calculate the recorded gravity values, and a discussion on the potential sources of error are identified. The calculations made to obtain Bouguer gravity anomalies for each observation in the database are given and the data are gridded and demonstrated visually. The varying methodology of recording the heights of the observations in the database is identified and accuracy estimates are assigned to the observation points.

Chapter 5 : describes what is meant by a global gravity model the different types and their use to determine quasigeoid height anomalies and gravity anomalies. Thirty seven satellite only, and nine combined global gravity models are compared to leveling and GNSS derived quasigeoid height anomalies and the terrestrial gravity anomalies. Of the thirty seven satellite only models, the model GO_CONS_GCF_2_DIR_R5 fits both the leveling and GNSS derived quasigeoid height anomalies and the terrestrial gravity data best. Of the nine combined global gravity anomaly models, EGM2008 fits the terrestrial gravity the best and Eigen-6C4 fit the leveling and GNSS derived quasigeoid height 
anomalies the best.

Chapter 6 : describes what airborne gravimetry is, gives a description of the national airborne gravimetry survey and then provides details of the apparatus used to collect the gravity measurements. It goes on to describe the procedure taken to reduce the raw data to Bouguer gravity anomalies along each flight line. Unfortunately the flight conditions for the data collection were not always ideal and a description is given of the steps taken to identify and remove erroneous data. The flight lines were also susceptible to mean offsets, details are given regarding the steps to remove these offsets. An accuracy assessment of the data is provided looking at the difference of data at flight line intersection points, and the data along two flight lines which were flown multiple times each are compared.

Chapter 7 : describes the method of least squares collocation and how it can be appropriately used to combine gravity anomaly observations of different quality. The airborne and terrestrial gravity data are first combined using this method and several calculations are made to properly account for the errors in the data. Two new data sets are introduced, the shipborne gravity data and a satellite altimetry dataset. All four data sets are combined into a single grid by least squares collocation and the results are demonstrated.

Chapter 8 : describes the steps taken to obtain a gridded Faye anomaly which will be used to determine a new gravimetric quasigeoid. A recap on the methodology of regional quasigeoid computation is given and the steps are applied to the gridded Faye anomaly with Eigen-6C4 acting as the reference signal for the remove and restore stages of the computation. Eighty different quasigeoids are computed, each of which has a different set of parameters used during the computation. Each of the 80 quasigeoids are compared to the leveling and GNSS derived quasigeoid height anomalies, used to evaluate NZGeoid2009, and the best fitting quasigeoid is determined. The difference between the best fitting quasigeoids and the leveling and GNSS derived quasigeoid height anomalies is evaluated in New Zealand's main urban areas and these results are compared to the current official national quasigeoid and are demonstrated to be better. The best fitting quasigeoid is finally compared to a newly available dataset of leveling and GNSS derived quasigeoid height anomalies which contains significantly more measurements. The new data are shown to demonstrate a similar agreement as the previous smaller data set of leveling/GNSS data; however it contains outliers which are shown to degrade the accuracy evaluation.

Chapter 9 : gives a summary of each stage of this thesis, the theoretical background, historical setting and the steps taken to compute a new gravimetric quasigeoid. The key research outcomes of the thesis are highlighted and attention is given to what could be done in the future to improve the computational steps taken to compute the gravimetric quasigeoid, its underlying data and its accuracy assessment.

Appendix A : gives a short report on an experiment which was undertaken to estimate two constants used to convert the millivolt accelerometer readings from the airborne gravimeter to $\mathrm{mGal}$. The determined values are crucial to evaluate the tilt correction when processing the airborne data in chapter 6 . 
Appendix B : describes a process to estimate the standard deviation of some empirical data which contains large outliers. The standard approach is heavily influenced by values on the tails of an empirical distribution and the method described here appropriately removes their influence. This method has been used throughout this work and is shown to be crucial to accurately estimate empirical standard deviations with the type of data (leptokurtotic) examined in this thesis.

Appendix C : describes the contents of the electronic appendices. Each of the raw gravity datasets which have been used are made available. The least squares collocation grids are provided for the examples shown in chapter 7 . The Faye and residual gravity anomaly grid and all of the calculated residual quasigeoids of chapter 8 are given with the scripts to determine the optimal parameter combination. Finally, the optimal quasigeoid is given in xyz and a Matlab format along with the scripts to compare the data to the GNSS/leveling derived quasigeoid heights. 


\section{Glossary}

\begin{tabular}{|c|c|}
\hline Abbreviations & \\
\hline $\mathrm{CDF}$ & Cumulative distribution function \\
\hline $\mathrm{cm}$ & Centimetres \\
\hline covar & Covariance \\
\hline DEM & Digital elevation model \\
\hline DGNSS & Differential Global Positioning System \\
\hline EGM2008 & Earth gravity model 2008 \\
\hline GA & Geoscience Australia \\
\hline GBVP & Geodetic Boundary Value problem \\
\hline GGM & Global gravity model \\
\hline GNSS & Global navigation satellite systems \\
\hline GNSS & Global positioning system \\
\hline GRS80 & Global reference system 1980 \\
\hline IAG & International association of geodesy \\
\hline $\mathrm{km}$ & kilometres \\
\hline LSC & Least squares collocation \\
\hline LVD & Local vertical datum \\
\hline $\mathrm{m}$ & metres \\
\hline mGal & milliGal \\
\hline NOAA & National Oceanic and Atmospheric Administration \\
\hline NZGeoid05 & New Zealand Quasigeoid 2005 \\
\hline NZGeoid2009 & New Zealand Quasigeoid 2009 \\
\hline NZVD2009 & New Zealand vertical datum 2009 \\
\hline $\mathrm{RCR}$ & Remove Compute Restore \\
\hline RMS & Root mean squared \\
\hline $\mathrm{s}$ & seconds \\
\hline STD & Standard deviation \\
\hline UNCLOS & United Nations Conventions on the Laws of the Sea \\
\hline var & Variance \\
\hline Symbols & \\
\hline$(r, \theta, \lambda)$ & Geocentric polar coordinates \\
\hline$a$ & Ellipsoidal semi major axis \\
\hline$b$ & Ellipsoidal semi minor axis \\
\hline$C$ & Geopotential number \\
\hline$\Delta g$ & Gravity anomaly \\
\hline$\Delta g_{B}$ & Bouguer gravity anomaly \\
\hline$\delta g_{B S}$ & Bouguer Slab effect \\
\hline$\Delta g_{\text {Eot }}$ & Eotvos correction \\
\hline$\Delta g_{F A}$ & Free Air gravity anomaly \\
\hline$\delta g_{F A}$ & Free Air Correction \\
\hline$\Delta g_{L}$ & Long wavelength gravity anomalies \\
\hline
\end{tabular}




$\mid \begin{aligned} & \Delta g_{m} \\ & \Delta g_{n} \\ & \Delta g_{R E S} \\ & \delta g_{T C} \\ & \Delta g_{\text {tilt }} \\ & \Delta g_{z} \\ & \Delta H_{m} \\ & \Delta H_{n} \\ & \Delta H_{z} \\ & e^{2} \\ & f \\ & g \\ & \bar{g} \\ & \gamma \\ & G M \\ & h \\ & H \\ & H^{N} \\ & H^{N-O} \\ & H^{o r t h} \\ & J_{2} \\ & k \\ & K(\psi) \\ & L \\ & N^{2} \\ & N_{\text {ind }} \\ & N_{L} \\ & N_{r e s} \\ & P_{n, m} \\ & \phi \\ & \psi \\ & \psi_{0} \\ & \rho \\ & S_{F}(\psi) \\ & S_{M}(\psi) \\ & S_{W G}(\psi) \\ & S_{V K}(\psi) \\ & S_{H G}(\psi) \\ & S_{F}(\psi) \\ & T \\ & \\ & \\ & \\ & \end{aligned}$

Gravity anomaly difference in mean tide system

Gravity anomaly difference in tide-free system

Residual gravity anomalies

Terrain Correction

Tilt correction

Gravity anomaly difference in zero tide system

Height difference in mean tide system

Height difference in tide-free system

Height difference in zero tide system

First eccentricity squared

Ellipsoidal flattening

Absolute gravity

Mean gravity along the plumbline

Normal gravity

gravitational constant time the mass of the Earth

ellipsoidal heights

Physical heights

Normal height

Normal-orthometric heights

Orthometric Heights

Second dynamic form factor

normal gravity constant

Truncation error kernel

Stokes kernel modification degree

Geoid separation from the ellipsoid

Primary indirect topographic effect

Long wavelength geoid undulations

Residual geoid undulations

Fully normalised associated Legendre functions

Latitude, in the context of gravity corrections

Spherical distance

Spherical integrations cap Stokes kernel

density

Stokes kernel

Meissel modified Stokes kernel

Wong and Gore modified Stokes kernel

Vanicek and Kleusberg modified Stokes kernel

Heck and Gruninger modified Stokes kernel

Featherstone modified Stokes kernel

Disturbing potential field

Gravitational potential field of the Earth approximating Ellipsoid

Gravitational potential field of the Earth

Angular velocity

Quasigeoid separation from the ellipsoid 


\section{Chapter 1}

\section{Heights and Reference Surfaces}

\subsection{Introduction}

In this chapter the concept of height is defined conceptually and mathematically. Heights must be referenced to a known surface. Commonly used reference surfaces are the ellipsoid, telluroid, quasigeoid and geoid. Each of these is defined explicitly in section 1.2. Section 1.3 specifies the distinction between geometrical height systems (which are not related to the Earth's gravity field) and physical height systems (which are based on geopotential numbers). The advantages/disadvantages of each are identified and the types of measurements needed to establish the heights are discussed.

\section{$1.2 \quad$ Height reference surfaces}

The ellipsoid: An ellipsoid is a three dimensional quadratic surface. When approximating the shape of the Earth an oblate ellipsoid of revolution is used. Oblate ellipsoids of revolution can be parameterized by two lengths, the semi-major (a) and semi-minor (b) axes. The semi-minor axis corresponds to the distance between the centre of the ellipsoid and the pole and the semi-major axis corresponds to the distance between the centre of the ellipsoid and the equator, this can be seen in figure 1.1.

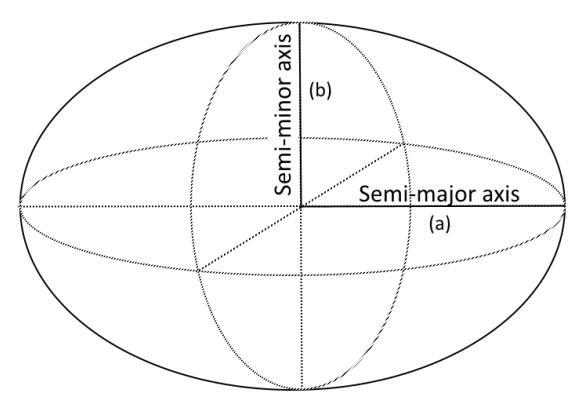

Figure 1.1: An ellipsoid with its two principal parameters - the semi-major (a) and semiminor (b) axes. 
The GRS80 (Moritz, 1980) reference ellipsoid has the following geometric parameters,

- Semi-major axis $(a)$ : 6378137 metres

- Semi-minor axis $(b)$ : 6356752.31 metres.

Since the GRS80 ellipsoid is used to approximate the Earth, it also has the following associated physical constants,

- Gravitational constant $(G M): 3986005 \times 10^{8} \mathrm{~m}^{3} \mathrm{~s}^{-2}$

- Angular velocity $(\omega): 7292115 \times 10^{-11} s^{-1}$.

- Second dynamic form factor $\left(J_{2}\right):=\frac{2(a-b)}{3 a}-\frac{\omega^{2} a^{2}}{3 G M}=108263 \times 10^{-8}$

The telluroid: The telluroid is defined in terms of the gravitational potential field of a reference ellipsoid, $U$, and the gravitational potential of the Earth, $W$ as follows.

For each point $P$ on the surface of the Earth, the gravitational potential of the Earth has the value $W_{P}$. At the point $P$ there is a curved line which passes through $P$ and is normal to equipotential surfaces in the potential field of the ellipsoid. Along this line, there is another point $Q$ where the gravitational potential, $U$, of the ellipsoid is equal to $W_{P}$. i.e. $U_{Q}=W_{P}$. For all points $P$ on the surface of the Earth there are corresponding points $Q$ which form a surface called the telluroid (Featherstone and Kuhn, 2006). The telluroid surface can be seen in figure 1.4.

The quasigeoid: The quasigeoid is also defined in terms of the gravitational potential field of a reference ellipsoid, $U$, and the gravitational potential of the Earth, $W$.

Here, the distance between $Q$ and $P$ along the curved line which passes through them is denoted $\xi$. For each point $P$ there is a corresponding value of $\xi$ which defines the quasigeoid separation from the ellipsoid at the point the curved line passes through the ellipsoid surface. The quasigeoid surface can also be seen in figure 1.4 (Featherstone and Kuhn, 2006).

The geoid: The geoid is defined in terms of the gravitational potential field of the Earth. Within the Earth's potential gravity field there are equipotential surfaces (i.e. where the gravitational potential is constant). A body of water on one of these equipotential surfaces would not flow anywhere since fluids flow from low potential numbers to high ones.

If the oceans on the Earth were under the influence of the Earth's gravity and rotation alone (i.e. no tides due to the sun and moon and no surface topography) the surface of the water body would lie on an equipotential surface which is termed the geoid (Heiskanen and Moritz 1967). For the gravitational potential field of the Earth $W$ the geoid is denoted $W_{0}$. The geoid surface can be extended over land hypothetically by considering thin canals cut through the landforms which the oceans would fill. The vertical component of gravity points normal to the surface of the geoid. 
Comparing the geoid to the idealized Earth given by the reference ellipsoid, wherever the vertical component of gravity of the Earth is larger than that of the reference ellipsoid the geoid is higher than the reference ellipsoid, and conversely, wherever the vertical component of gravity of the Earth is less than that of the reference ellipsoid the geoid is lower than the reference ellipsoid. These two scenarios correspond to mass excesses and mass deficits respectively i.e. the shape of the geoid is governed by the irregularity of mass distributions within the Earth.

\subsection{Height systems}

There are two forms of height systems. The first is purely geometrical and is typically based on a reference ellipsoid. The second are physical height surfaces which are based on geopotential numbers, these are useful from an engineering perspective since they accurately predict the flow of water.

\subsubsection{Geometric height systems}

The ellipsoidal height (h) of an observation point is given by the distance of the point to the surface of some defined ellipsoid, along a path which is normal to the ellipsoid surface as shown in figure 1.2. They are strictly a geometric value that is usually obtained from global navigation satellite systems (GNSS).

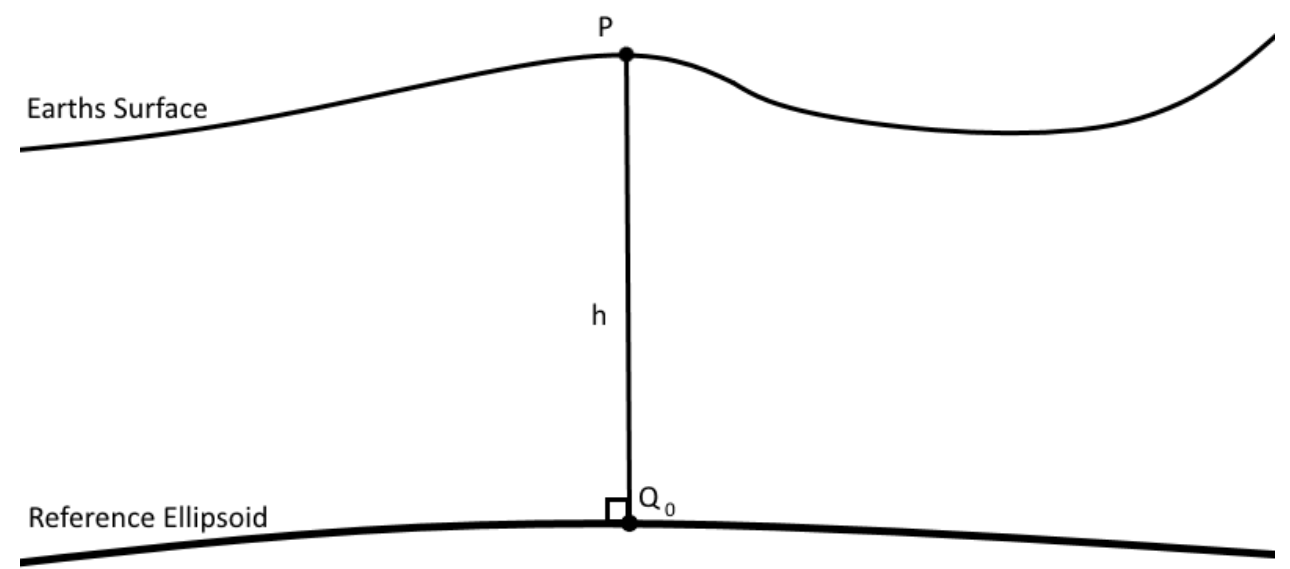

Figure 1.2: The separation between points $Q_{0}$ (which is on the reference ellipsoid) and $P$ (on the surface of the Earth).

The ellipsoidal heights are not directly related to the Earth's gravity field and so, although they are easy to compute, they are not practical from an engineering and surveying perspective as they do not accurately predict the flow of water. 


\subsubsection{Physical height systems}

All physical height reference systems are inherently based on geopotential numbers (Amos, 2007). These height systems can be used to predict the flow of water since it will always flow from a low potential value to a high one.

For $W$ the gravitational potential field of the Earth, let $W_{A}$ be an equipotential surface within the field and consider a point, $\mathrm{B}$ (above $W_{A}$ ) which lies on another equipotential surface $W_{B}$. The height of the point $B$ above the reference surface $W_{A}$ is calculated from the geopotential number $C$ given by

$$
C=W_{A}-W_{B}=\int_{A}^{B} g d z .
$$

$d z$ is an infinitesimally small step along the path, called the plumbline, between the surface $W_{A}$ and point $\mathrm{B}$. The plumbline points in the direction normal to every equipotential surface in the potential field $W$ as can be seen in figure 1.3.

Since $g$ is a continuous function along the path from $A$ to the point $B$ the first mean value theorem for integration implies that there is a point $x$ along the path such that $g(x)=\bar{g}$, which is the integral average value of $g$ over the path from $A$ to $B$ i.e.

$$
\bar{g}=\frac{\int_{A}^{B} g d z}{\int_{A}^{B} d z} .
$$

The height $H$ of the point $B$ above the reference surface $W_{A}$ is then given by,

$$
H=\frac{C}{\bar{g}} .
$$

$W$ and hence $C$ are typically given in terms of $\mathrm{m}^{2} / \mathrm{s}^{2}$ and $\bar{g}$ is given in terms of $\mathrm{m} / \mathrm{s}^{2}$. The units of the height $H$ are then purely in terms of length. In general, the form of equation 1.3 is used as a basis to define all height systems based on geopotential numbers.

Orthometric heights - based on the geoid: The equipotential surface $W_{0}$ which best agrees with mean sea level is called the geoid. It is the shape that the Earth's oceans would take when under the influence of gravity alone. Where $W_{0}$ is the reference surface and $P$ is some point on or above the Earth, the geopotential number $C$ is given by,

$$
C=W_{0}-W_{P}
$$

The orthometric height, $H^{\text {orth }}$, is defined as the distance between a point $P$ in the field (above $W_{0}$ ) and a corresponding point $P_{0}$ on the surface of the geoid, along the plumbline. 


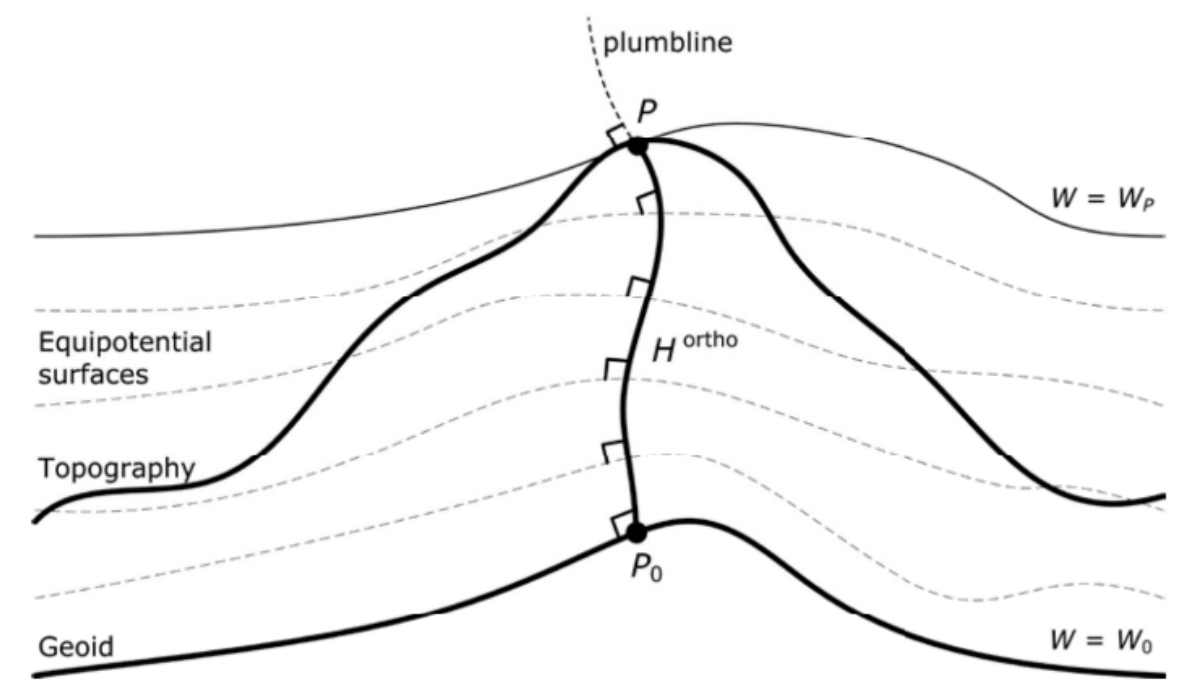

Figure 1.3: The separation between points $P_{0}$ (which is on the geoid) and $P$ defined as the distance along the plumbline. - from Featherstone and Kuhn 2006

Equation 1.3 gives

$$
H^{\text {orth }}=\frac{C}{\bar{g}},
$$

with $\bar{g}$ is the mean value of the gravitational acceleration, $g$, along the plumbline.

The geoid surface is typically under the terrain. This means orthometric heights are impossible to directly compute since, to accurately compute $\bar{g}$ would require knowledge of the precise variations in gravitational acceleration and the exact mass-density distribution along the plumbline through the topography.

However, several approximations do exist such as Helmert (1890) orthometric heights, Mader (1954) orthometric heights and Neithammer (1932) orthometric heights which are as follows:

Helmert (1890) orthometric heights approximate $\bar{g}$ as,

$$
\bar{g}=g_{s}+\frac{1}{2} \frac{\partial \gamma}{\partial h} H^{\text {orth }}-2 \pi G M \rho H^{\text {orth }}
$$

where $g_{s}$ is the observed absolute gravity at the surface of the Earth, $\frac{\partial \gamma}{\partial h}$ is the vertical gradient of the normal gravity and $\rho$ is the density of the topography (Amos, 2007).

Here, the mean gravity is easy to compute. However the topography is considered to be satisfactorily approximated by a slab (see section 2.2.3) and $\rho$ is taken to be constant. These assumptions mean the resulting orthometric height can significantly differ from the true orthometric height. 
Neithammer (1932) orthometric heights approximate $\bar{g}$ similarly to Helmert but with the addition of two extra terms $\partial g^{T}$ and $\bar{\partial} g^{T}$.

$$
\bar{g}=g_{s}+\frac{1}{2} \frac{\partial \gamma}{\partial h} H^{\text {orth }}-2 \pi G M \rho H^{\text {orth }}+\partial g^{T}-\bar{\partial} g^{T}
$$

$\partial g^{T}$ is the terrain effect (as discussed in section 2.2.3) and $\bar{\partial} g^{T}$ is the integral mean terrain effect along the plumbline.

This approximation takes into account topographic variations from the slab. However this can be computationally expensive (as seen in section 2.2.3) and again $\rho$ is still taken to be constant which leads to inaccuracies.

Mader (1954) orthometric heights approximate $\bar{g}$, again similarly to Helmert but with two extra terms $\partial g^{T}$ and $\partial g_{0}^{T}$.

$$
\bar{g}=g_{s}+\frac{1}{2} \frac{\partial \gamma}{\partial h} H^{\text {orth }}-2 \pi G M \rho H^{\text {orth }}+\frac{\partial g^{T}-\partial g_{0}^{T}}{2}
$$

$\partial g^{T}$ is again the terrain effect at the topographic surface as discussed in section 2.2.3 and $\partial g_{0}^{T}$ is the terrain effect at the surface of the geoid (Amos, 2007).

As with the Neithammer method, computing the terrain corrections can be computationally expensive and again $\rho$ is still taken to be constant which leads to inaccuracies. 
Normal heights - based on the telluroid: Consider an ellipsoid which best fits the gravitational field of the Earth in terms of the total mass and rotation. It has potential gravity field $U$ and there is an equipotential surface $U_{0}$ in the potential field such that $U_{0}=W_{0}$. The normal height, as shown in figure 1.4, is given by changing the divisor in equation 1.3 to a value, $\bar{\gamma}$, determined from the normal gravity field, $\gamma$, generated by the ellipsoid. i.e. in terms of equation 1.3,

$$
H^{N}=\frac{C}{\bar{\gamma}}
$$

and

$$
\bar{\gamma}=\frac{1}{H^{N}} \int_{0}^{H^{N}} \gamma d h
$$

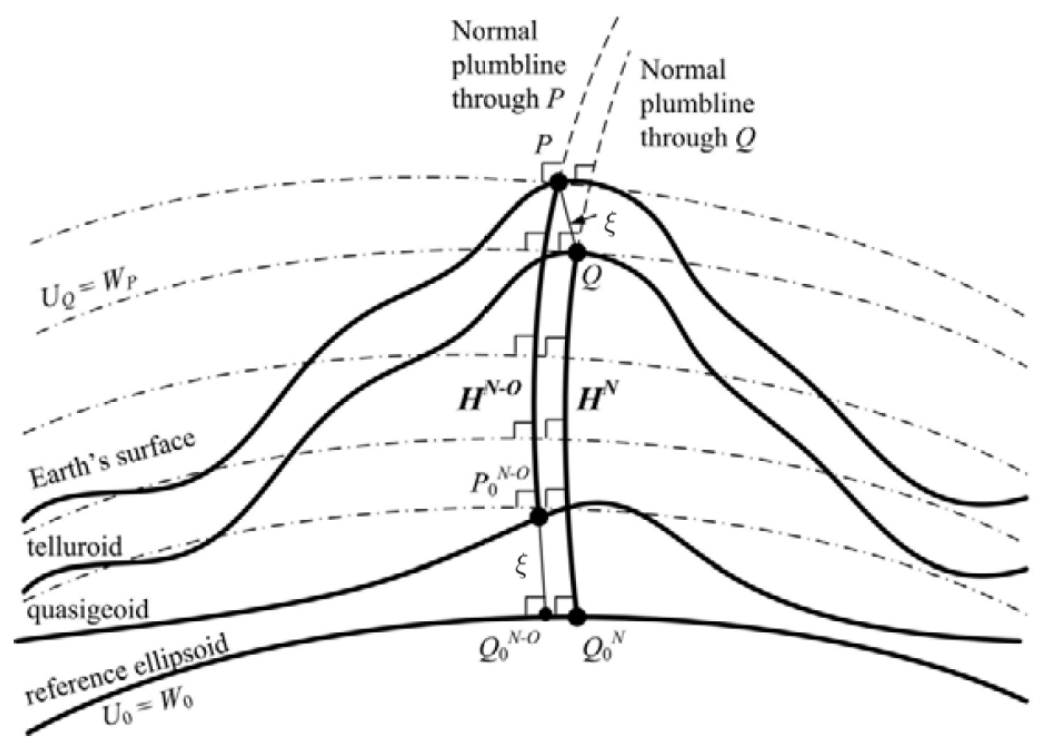

Figure 1.4: The concept of normal heights $H^{N}$ and normal-orthometric heights $H^{N-O}$ from Featherstone and Kuhn (2006). Plumblines shown here are separated horizontally for illustrative purposes only.

For a point $P$ on the surface of the Earth, $\bar{\gamma}$ is the average gravity on the path along the ellipsoidal surface normal (which passes through the point $P$ ) between a point $Q_{0}^{N}$ (on the surface of the ellipsoid) and a point $Q$. The point $Q$ is such that $U_{Q}=W_{P}$.

As discussed previously, for all points $P$ taken on the surface of the Earth the corresponding points $Q$ form a surface which is known as the telluroid. The distance from $Q$ to $P$ is called the height anomaly $\xi$. It is determined from ellipsoidal height $h$ by,

$$
\xi=h-H^{N} .
$$


Normal-Orthometric heights - based on the quasigeoid: Normal-orthometric heights are defined as the distance between a point $P_{0}$ on the quasigeoid and a point $P$ on the surface of the Earth which both lie along a path normal to the ellipsoidal potential gravity field.

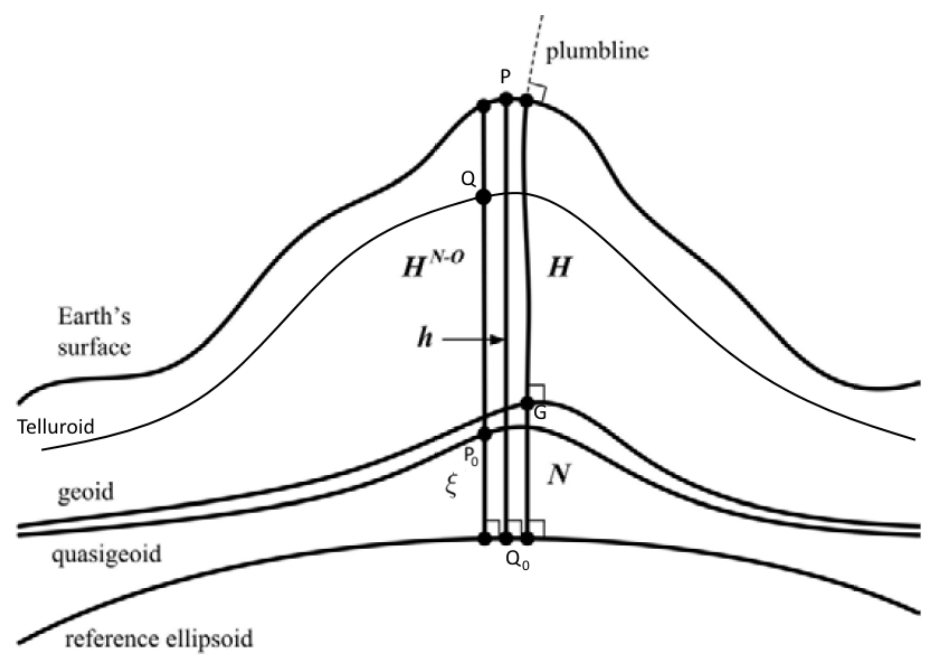

Figure 1.5: Ellipsoidal, orthometric and normal-orthometric heights and the geoid undulation $N$ and height anomaly $\xi$-adapted from Amos (2007). Again plumblines are separated horizontally for clarity.

This time in equation $1.1 C$ becomes $C^{\prime}$ where

$$
C^{\prime}=U_{0}-U_{P}=\int_{P_{0}}^{P} \gamma d h
$$

so that,

$$
H^{N-O}=\frac{C^{\prime}}{\bar{\gamma}}
$$

Where $\bar{\gamma}$ is the average gravity on the path between point $P_{0}$ and $P$. The path follows the ellipsoidal surface normal between a point $Q_{0}$ (on the surface of the ellipsoid) and the point $P$ and passes through $P_{0}$. Again the height anomaly $\xi$ is determined from ellipsoidal height $h$ by,

$$
\xi=h-H^{N-O} \text {. }
$$

The distance from $Q_{0}$ to $P_{0}$ is the same as the distance between $Q$ and $P$ over the ellipsoidal surface normal path, which is the same as saying that the distance between the surface of the ellipsoid and the quasigeoid is the same as the distance between the Telluroid and the surface of the Earth.

The main advantage of using a normal-orthometric height system is that once a quasigeoid surface has been established the height at any location on the Earth's surface can be simply calculated from the ellipsoidal height $h$ and the normal (ellipsoidal) gravity field $\gamma$. However the quasigeoid is not truly an equipotential surface of the Earth's gravity field and so will not predict the flow of fluids with complete reliability. The geoid (true 
equipotential surface) and quasigeoid differ most in high topography where the contribution to $\bar{g}$ will be largest. Rapp (1997) and Sjberg (1995) show that the differences reach up to $3.4 \mathrm{~m}$ in the Himalayas and Amos and Featherstone (2003) investigated this in the $\mathrm{NZ}$ region and showed that the difference can be up to $0.5 \mathrm{~m}$ at Aoraki/Mt Cook.

\subsection{Summary}

This chapter gives a definition of the ellipsoid, telluroid, quasigeoid and geoid reference surfaces, which will frequently be referred to throughout the remainder of this text.

The distinction between physical heights and geometrical heights is given. Geometrical heights are easy to obtain from GNSS, but unlike physical heights, they cannot be used to predict the flow of water and so are not meaningful from an engineering or surveying perspective.

Physical heights have been defined mathematically in terms of geopotential numbers. Three physical height systems have been identified; Orthometric heights (which are based on the geoid but impossible to accurately compute without knowledge of the precise variations in gravitational acceleration and the exact mass-density distribution along the plumbline through the topography), Normal heights and Normal-Orthometric heights (which are based on the telluroid and quasigeoid respectively and only require a GNSS and the normal gravity field to compute). 


\section{Chapter 2}

\section{The Disturbing Potential, Gravity Anomalies and Geoid Heights}

\subsection{Introduction}

This chapter introduces the concept of the disturbing gravitational potential, which is the difference between the gravitational potential of an Earth approximating ellipsoid and the true gravitational potential of the Earth. Geoid heights determined from the disturbing potential, by Bruns (1878) equation, and their relationship to gravity anomalies is specified.

The necessary calculations to obtain the gravity anomalies, needed to determine the geoid heights, from absolute gravity observations are discussed. This involves calculating the absolute gravity of the Earth approximating ellipsoid at the location of the observed gravity value, and also determining the gravitational effect of topography.

The effect of making gravity observations at varying elevations is discussed and methods to upward/downward continue gravity observations are given.

The permanent (non periodic) tidal effects of the sun and moon on the gravitational potential field of the Earth is covered. The internationally preferred tidal model is specified and transformations between different tidal models are given.

Finally, the process to transform gridded gravity values into geoid heights by Stokes' integral and Helmert's second method of condensation are discussed in detail. 


\subsection{The disturbing potential and gravity anomalies}

Following Heiskanen and Moritz (1967), for $W$ the potential field of the Earth and $U$ the potential field of the reference ellipsoid, define the disturbing potential $T$ as follows,

$$
T=W-U \text {. }
$$

For a point $G=\left(\theta_{G}, \lambda_{G}\right)$ on the geoid,

$$
T_{G}=W_{G}-U_{G}
$$

Bruns' (1878) equation gives the geoid undulation (which is the separation between the geoid and the ellipsoid) at the point $Q_{0}=\left(\theta_{0}, \lambda_{0}\right)$ on the ellipsoid surface as,

$$
N=\frac{T_{G}}{\gamma_{Q_{0}}}
$$

where $\gamma_{Q_{0}}$ is the normal gravity (see section 2.2.1) at the point $Q_{0}$ which is on the reference ellipsoid. For a point $P=(r, \theta, \lambda)$ on the surface of the Earth,

$$
T_{P}=W_{P}-U_{P}
$$

Analogously, the gravity disturbance at the point $P$ is defined by,

$$
\delta g_{P}=g_{P}-\gamma_{P}
$$

(Alberts, 2009). Under a spherical approximation to the gravity field, the disturbing potential is related to the gravity disturbance by the following,

$$
\frac{d T}{d r}=-\delta g
$$

Classically, gravity anomalies $\Delta g$ at a point $G$ on the geoid are given by,

$$
\Delta g_{G}=g_{G}-\gamma_{Q_{0}}
$$

where $Q_{0}$ is on the surface of the reference ellipsoid (i.e. no free air correction is used as discussed in section 2.2.2). The modern definition is instead given by,

$$
\Delta g_{P}=g_{P}-\gamma_{Q}
$$

where $P$ is on the surface of the Earth and $Q$ is on the Telluroid. This can be interpreted as a measured gravity value at $P$ minus the normal gravity calculated at a height above the reference ellipsoid given by the normal-orthometric height of the point $P$ (i.e. $\gamma_{Q}$ consists of the normal gravity and the free air effect as discussed in sections 2.2.2 and 2.2.1). When the orthometric height of the point $P$ is zero, the classical definition of the gravity anomaly and the modern definition are in agreement since $W_{0}=U_{0}$. In particular, over the oceans the point $P$ is on the geoid, so the quasigeoid and the geoid are then the same, i.e. $\xi=N$.

Again, under a spherical approximation, the gravity anomaly is related to the disturbing potential by the following equation,

$$
\frac{d T}{d r}+\frac{2}{r} T=-\Delta g
$$

This equation is known as the geodetic boundary-value problem (GBVP), Heiskanen and Moritz (1967). 


\subsubsection{Normal gravity}

Normal gravity is the absolute gravity of the Earth approximating ellipsoid (as discussed in section 1.2). It is dependent only on the latitude, $\phi$, at which it is evaluated and can be written in the following closed form (Moritz 1980);

$$
\gamma(\phi)=\gamma_{a} \frac{1+k \sin ^{2}(\phi)}{\sqrt{1-e^{2} \sin ^{2}(\phi)}}
$$

where (for the GRS80 reference ellipsoid as discussed in section 1.2), $\gamma_{a}=9.7803267715 \mathrm{~m} \mathrm{~s}^{-2}$ is the absolute gravity at the equator, $k=0.001931851353$ is the normal gravity constant and $e^{2}=1-b^{2} / a^{2}=0.00669438002290$ is the first eccentricity squared (where $a$ and $b$ are the semi-major and semi-minor axes of the reference ellipsoid).

\subsubsection{Free air effect}

The free air effect describes the reduction in the normal gravity when making an observation at some elevation $H$ above the geoid. The second order accurate effect given by Heiskanen and Moritz (1967) is,

$$
\delta g_{F A}=\frac{d \gamma}{d H}=\frac{2 \gamma}{a}\left(1+f+m(-3 f+5 / 2 m) \sin ^{2}(\phi)\right) H-\frac{3 \gamma_{a} H^{2}}{a^{2}} .
$$

Here, $f=(a-b) / a$ (for $a$ and $b$ the semi-major and semi-minor axes of the ellipsoid) and $m=0.00344978600308$ is the ratio between the gravitational and centrifugal forces at the equator. This formula corresponds to a spherical approximation to the shape of the Earth. Historically this correction has been reduced to a linear form given by,

$$
\tilde{\delta g_{F A}}=\frac{d \gamma}{d H}=-\frac{2 \gamma}{R} H \approx-0.3086 H\left(10^{-5} \mathrm{~m} / \mathrm{s}^{2}\right)
$$

Here, $R$ is the distance from the centre of mass of the spherical Earth and $H$ is given in metres and is the normal-orthometric height. Throughout this thesis, the second order free air correction will be used which is of the form of equation 2.10. The importance of the second order free air correction is discussed in Featherstone and Dentith (1997).

N.B. In general, when observed absolute gravity values have had the Normal gravity and free air effect removed the signal is very small, for this reason the units typically used are $\mathrm{mGal}$ (milli Gal) where $1 \mathrm{mGal}=10^{-5} \mathrm{~m} / \mathrm{s}^{2}$.

The free air anomaly is given by the following,

$$
\Delta g_{F A}=g-\gamma+\delta g_{F A}
$$




\subsubsection{Bouguer slab and terrain corrections}

The Bouguer slab and terrain corrections account for the gravitational effect of topography around an observation point. Correcting for this allows gravity observations to be used to study subsurface density variations.

Hammer (1939) gives the formula for the gravitational effect of an annulus of topography with an inner radius $r$, outer radius $R$ and height $h$ as,

$$
\delta g(r, R, h, \rho)=0.0419 \rho\left(R-r+\sqrt{r^{2}+h^{2}}-\sqrt{R^{2}+h^{2}}\right)
$$

(in the $x, y$ plane) where $\rho$ is the density. At the limit for $R \rightarrow \infty$ when $r=0$,

$$
\lim _{R \rightarrow \infty} \delta g=0.0419 \rho h
$$

When $h$ is taken to be the normal-orthometric height of the observation location, this is the expression for the infinite Bouguer slab of thickness $h$, denoted $\delta g_{B S}$. The total effect of terrain is computed as follows, $\delta g_{B S}$ is first subtracted and then the effect of the missing mass below the slab and additional mass above the slab is be corrected for; this is called the terrain correction $\delta g_{T C}$.

Over Corrected Terrain

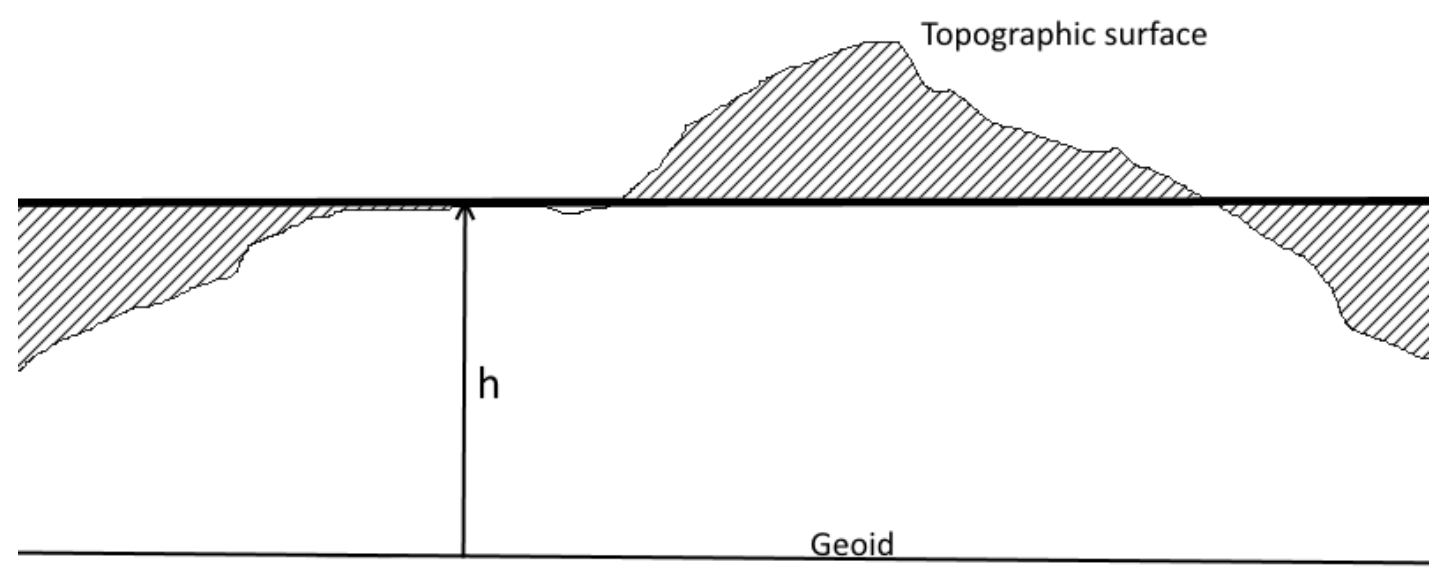

Figure 2.1: Bouguer slab and residual terrain.

When the total effect of the topography is subtracted from a free air gravity anomaly by the subtraction of the Bouguer slab and restoration of the residual terrain effect, the resulting gravity anomaly is termed the Bouguer anomaly, here denoted $\Delta g_{B}$.

$$
\Delta g_{B}=g-\gamma-\delta g_{F A}-\delta g_{B S}+\delta g_{T C}
$$


Historically, when a gravity observation is made in the field, the topographic variations around the measurement locations are estimated visually and the mean height (relative to where the observation was made) in sectors of specified annuli are recorded. The separate annuli are known as Hammer zones and each is divided into a number of sectors. In the far field it is difficult to estimate the topography by eye and so the zones are laid out on top of a topographic map and the mean height difference in each sector is estimated.

\begin{tabular}{cccc}
\hline Zone & $\begin{array}{c}\text { Inner } \\
\text { Radius }\end{array}$ & $\begin{array}{c}\text { Outer } \\
\text { Radius }\end{array}$ & $\begin{array}{c}\text { Number of } \\
\text { Compartments }\end{array}$ \\
\hline A & 0.0 & 2.0 & 1 \\
B & 2.0 & 16.6 & 4 \\
C & 16.6 & 53.3 & 6 \\
D & 53.3 & 170.1 & 6 \\
E & 170.1 & 390.1 & 8 \\
F & 390.1 & 894.9 & 8 \\
G & 894.9 & 1530 & 12 \\
H & 1530 & 2615 & 12 \\
I & 2615 & 4469 & 12 \\
J & 4469 & 6653 & 16 \\
K & 6653 & 9903 & 16 \\
L & 9903 & 14,742 & 16 \\
M & 14,742 & 21,944 & 16 \\
\hline
\end{tabular}

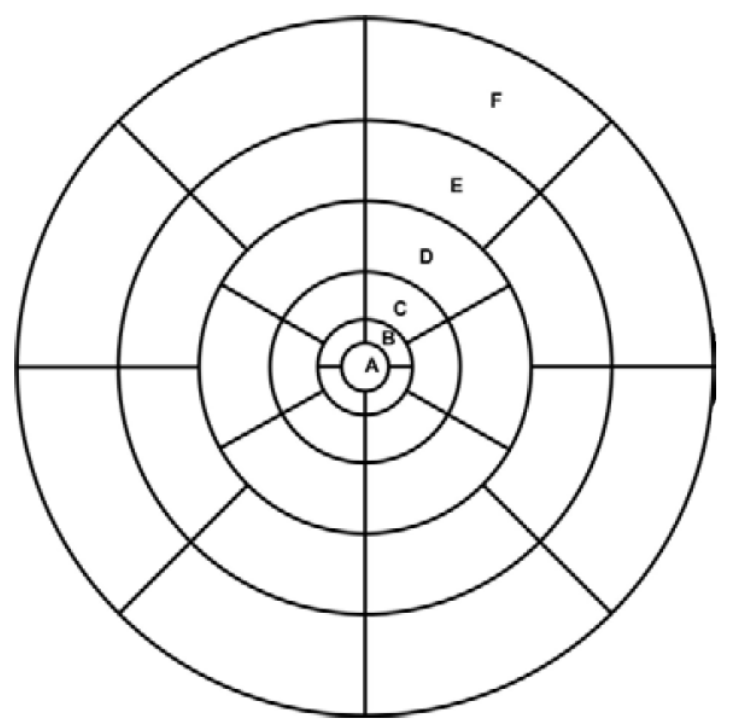

Figure 2.2: Left shows the hammer zones radii in $\mathrm{m}$ and number of compartments and Right shows the geometry of the zones. Figure has been adapted from Amos (2007).

The accuracy of this method largely relies on how well the mean topographic height can be estimated (either by eye or from maps) and also the number of sectors in each annulus (Nowell, 1999).

Using a digital elevation model (DEM) and a prism approximation of the topography, the estimated mean topographic height is greatly improved and the "number of sectors" can be increased up to the resolution of the DEM. The limitations in the accuracy of terrain corrections computed from a DEM are generally from the very near zone. For example, a digital elevation model with a resolution of $64 \mathrm{~m}$ will have no topographic variability between an observation site out to around $32 \mathrm{~m}$ and so the terrain correction for this range will be zero since the DEM is band limited (Kirby and Featherstone, 2002). In rough topography this can be problematic since the terrain corrections in the near zone can be up to several mGal. 
Terrain corrections computed using Nagy Prisms The Nagy (1966) prism method for making terrain corrections is performed by approximating the topography, given by a DEM, with rectangular prisms.

\section{Terrain Corrections Using Digital Computers}

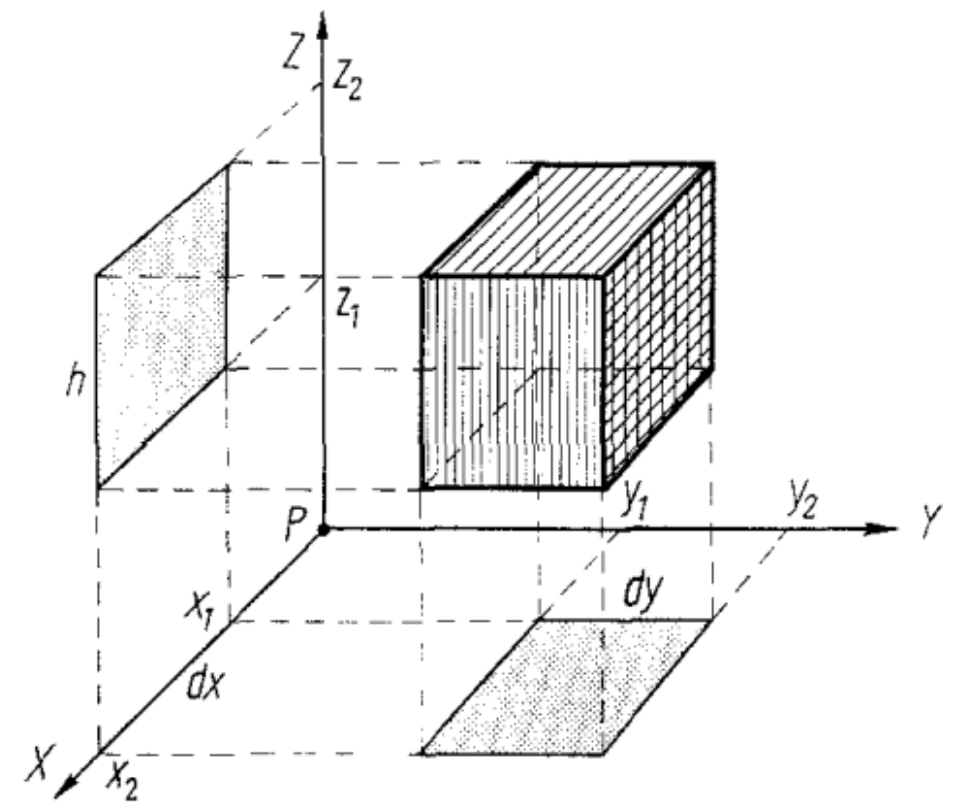

Figure 2.3: Terrain correction prism - adapted from Nagy (1966).

A piece of topography centred at the point $x_{p}, y_{p}$ can be approximated with a rectangular prism with an area of $\partial x \times \partial y$ and 4 corners in the $x, y$ plane, $\left(x_{p 1}, y_{p 1}\right),\left(x_{p 2}, y_{p 1}\right)$, $\left(x_{p 1}, y_{p 2}\right),\left(x_{p 2}, y_{p 2}\right)$ where,

$$
\begin{aligned}
& x_{p 1}=x_{p}-\frac{\partial x}{2} \\
& x_{p 2}=x_{p}+\frac{\partial x}{2} \\
& y_{p 1}=y_{p}-\frac{\partial y}{2} \\
& y_{p 2}=y_{p}+\frac{\partial y}{2} .
\end{aligned}
$$

The correction, $\delta g_{T C}$ for the vertical gravity component of each piece of terrain is given in Nagy, (1966). The coordinates $\left(x_{p}, y_{p}, z_{p}\right)$ are with respect to an observation made at the origin and Nagy's prism based terrain correction is calculated by the following closed formula, 


$$
\begin{gathered}
\delta g_{T C}=G \rho\left[x_{p 2}\left(\ln \left(\frac{y_{p 2}+\sqrt{x_{p 2}^{2}+y_{p 2}^{2}}}{y_{p 2}+\sqrt{x_{p 2}^{2}+y_{p 2}^{2}+z_{p}^{2}}}\right)-\ln \left(\frac{y_{p 1}+\sqrt{x_{p 2}^{2}+y_{p 1}^{2}}}{y_{p 1}+\sqrt{x_{p 2}^{2}+y_{p 1}^{2}+z_{p}^{2}}}\right)\right)-\right. \\
x_{p 1}\left(\ln \left(\frac{y_{p 2}+\sqrt{x_{p 1}^{2}+y_{p 2}^{2}}}{y_{p 2}+\sqrt{x_{p 1}^{2}+y_{p 2}^{2}+z_{p}^{2}}}\right)-\ln \left(\frac{y_{p 1}+\sqrt{x_{p 1}^{2}+y_{p 1}^{2}}}{y_{p 1}+\sqrt{x_{p 1}^{2}+y_{p 1}^{2}+z_{p}^{2}}}\right)\right)+ \\
y_{p 2}\left(\ln \left(\frac{x_{p 2}+\sqrt{x_{p 2}^{2}+y_{p 2}^{2}}}{x_{p 2}+\sqrt{x_{p 2}^{2}+y_{p 2}^{2}+z_{p}^{2}}}\right)-\ln \left(\frac{x_{p 1}+\sqrt{x_{p 1}^{2}+y_{p 2}^{2}}}{x_{p 1}+\sqrt{x_{p 1}^{2}+y_{p 2}^{2}+z_{p}^{2}}}\right)\right)- \\
y_{p 1}\left(\ln \left(\frac{x_{p 2}+\sqrt{x_{p 2}^{2}+y_{p 1}^{2}}}{x_{p 2}+\sqrt{x_{p 2}^{2}+y_{p 1}^{2}+z_{p}^{2}}}\right)-\ln \left(\frac{x_{p 1}+\sqrt{x_{p 1}^{2}+y_{p 1}^{2}}}{x_{p 1}+\sqrt{x_{p 1}^{2}+y_{p 1}^{2}+z_{p}^{2}}}\right)\right)+ \\
\arcsin \left(\frac{y_{p 2}^{2}+h^{2}+y_{p 2} \sqrt{x_{p 2}^{2}+y_{p 2}^{2}+h^{2}}}{\left(y_{p 2}+\sqrt{x_{p q}^{2}+y_{p 2}^{2}+h^{2}}\right) \sqrt{y_{p 2}^{2}+h^{2}}}\right)-\arcsin \left(\frac{y_{p 1}^{2}+h^{2}+y_{p 1} \sqrt{x_{p 2}^{2}+y_{p 1}^{2}+h^{2}}}{\left(y_{p 1}+\sqrt{x_{p 2}^{2}+y_{p 1}^{2}+h^{2}}\right) \sqrt{y_{p 1}^{2}+h^{2}}}\right)+ \\
\left.\left.\arcsin \left(\frac{y_{p 1}^{2}+h^{2}+y_{p 1} \sqrt{x_{p 1}^{2}+y_{p 1}^{2}+h^{2}}}{\left(y_{p 1}+\sqrt{x_{p 1}^{2}+y_{p 1}^{2}+h^{2}}\right) \sqrt{y_{p 1}^{2}+h^{2}}}\right)\right)\right]
\end{gathered}
$$

where $G$ is the gravitational constant and $\rho$ is the density and $h=\left|z_{p}-z_{0}\right|$ with $z_{0}$ the height at the origin. To obtain the terrain correction over some area $A$, the $\Delta g_{p}$ 's for all $x_{p}, y_{p}$ in the area are summed up.

Hammer prism approximation Hammer's (1939) formula can be adapted to calculate the effect of a piece of topography in the $(\mathrm{x}, \mathrm{y})$ plane as follows.

To correct for the effect of a piece of terrain at $x_{p}, y_{p}, z_{p}$ on an observation made at the origin choose the following,

$$
\begin{aligned}
& r=\sqrt{x_{p}^{2}+y_{p}^{2}}-\sqrt{\frac{\delta^{2}}{2}} \\
& R=\sqrt{x_{p}^{2}+y_{p}^{2}}+\sqrt{\frac{\delta^{2}}{2}}
\end{aligned}
$$

where $\delta$ is the resolution of the DEM. Then calculate a factor $f$ which corresponds to the ratio between the area of the piece of terrain and the area of the annulus,

$$
f=\frac{\delta^{2}}{\pi\left(R^{2}-r^{2}\right)}
$$


The terrain correction $\delta g_{T C}$ for the piece of terrain $x_{p}, y_{p}, h_{p}$ is then given by,

$$
\delta g_{T C}=f \delta g(r, R, h, \rho) .
$$

The geometry of this correction can be seen in figure 2.4.

\section{Hammer approximation to a prism}

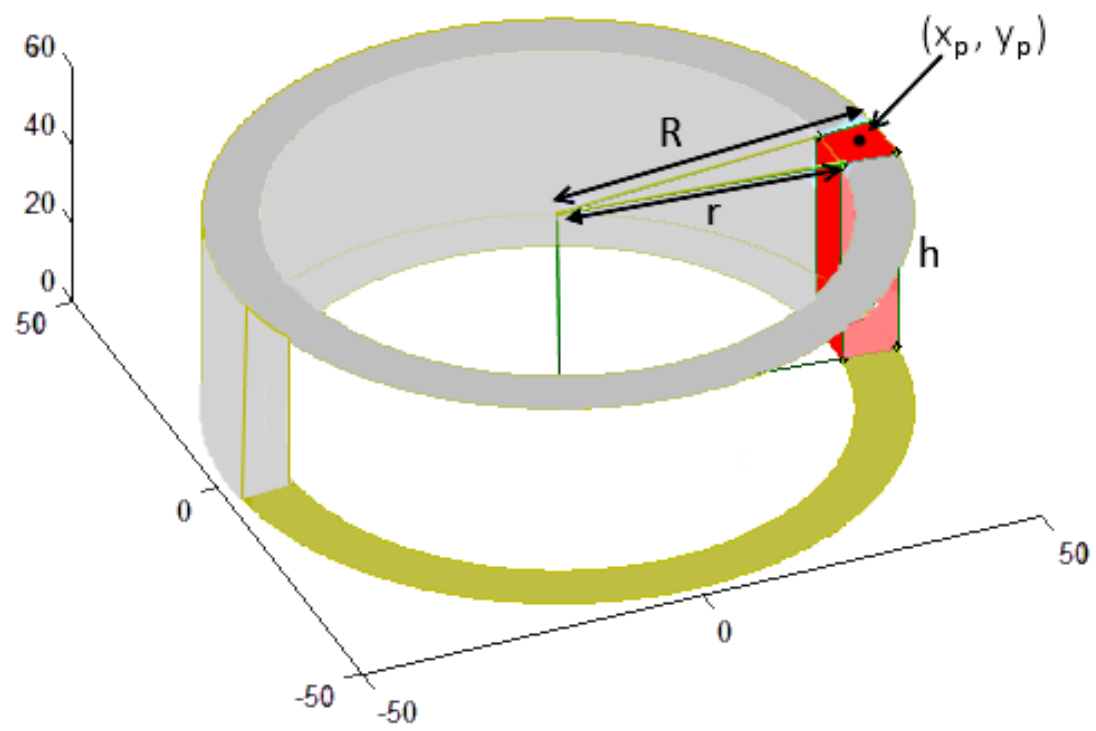

Figure 2.4: An illustration of the geometry for a segment of Hammer's terrain correction for an annulus of topography.

Conservatively, the Hammer calculation returns terrain corrections around 10 times faster. This is because the formula is simpler, i.e. less computational steps are needed. Moreover, the Nagy prism calculation appears to be sensitive to rounding errors caused by taking numerous square roots and logarithms; the Hammer method is less problematic in this respect.

A comparison of the Nagy prism and Hammer prism approximation methods for synthesised topography (namely a $100 \times 100 \mathrm{~m}$ piece of topography at distances from zero out to $2000 \mathrm{~m}$ from an observation point with heights taken at 10, 100 and $1000 \mathrm{~m}$ ) is shown in figure 2.5 and demonstrates that the difference between the two methods is negligible even in the very near zone of the observation point.

For example, in the very extreme case of a $1000 \mathrm{~m}$ tall piece of topography immediately next to an observation the difference between the two methods is $0.2 \mathrm{mGal}$. 


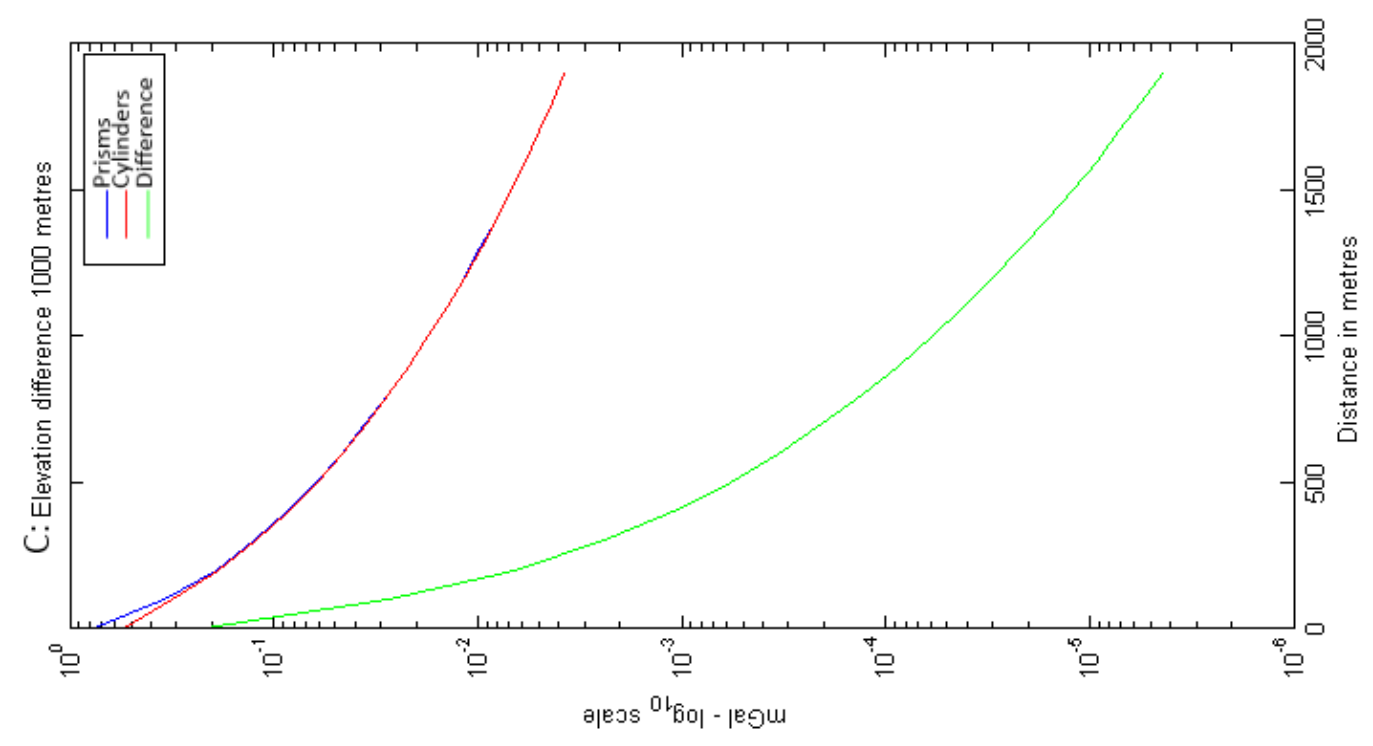

in

ธิ)

घี

疋

명

(9)응

를

है

है

20

है

它莡

잉

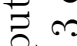

द्वे

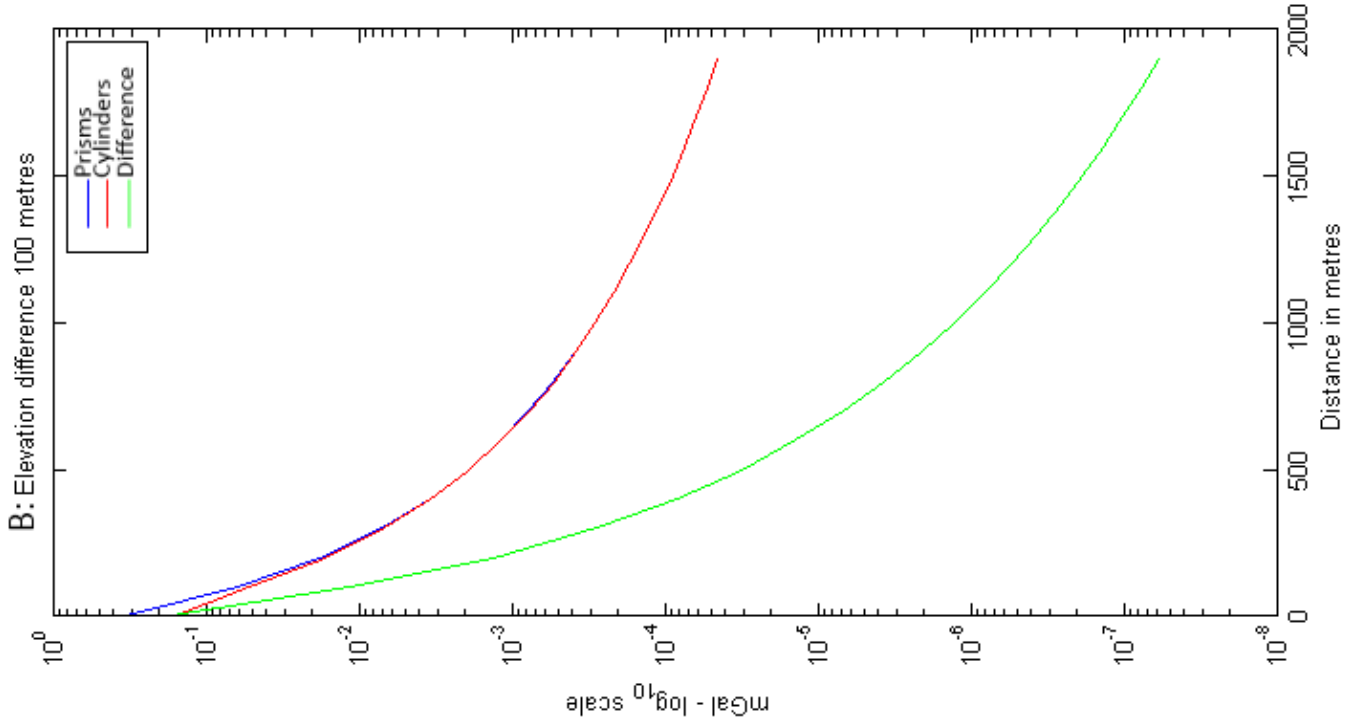

ลิํํㅇ

त्र

ธิธ

$\stackrel{2}{\circ}$

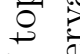

पै

80

足,

ఠี

N

है

\%

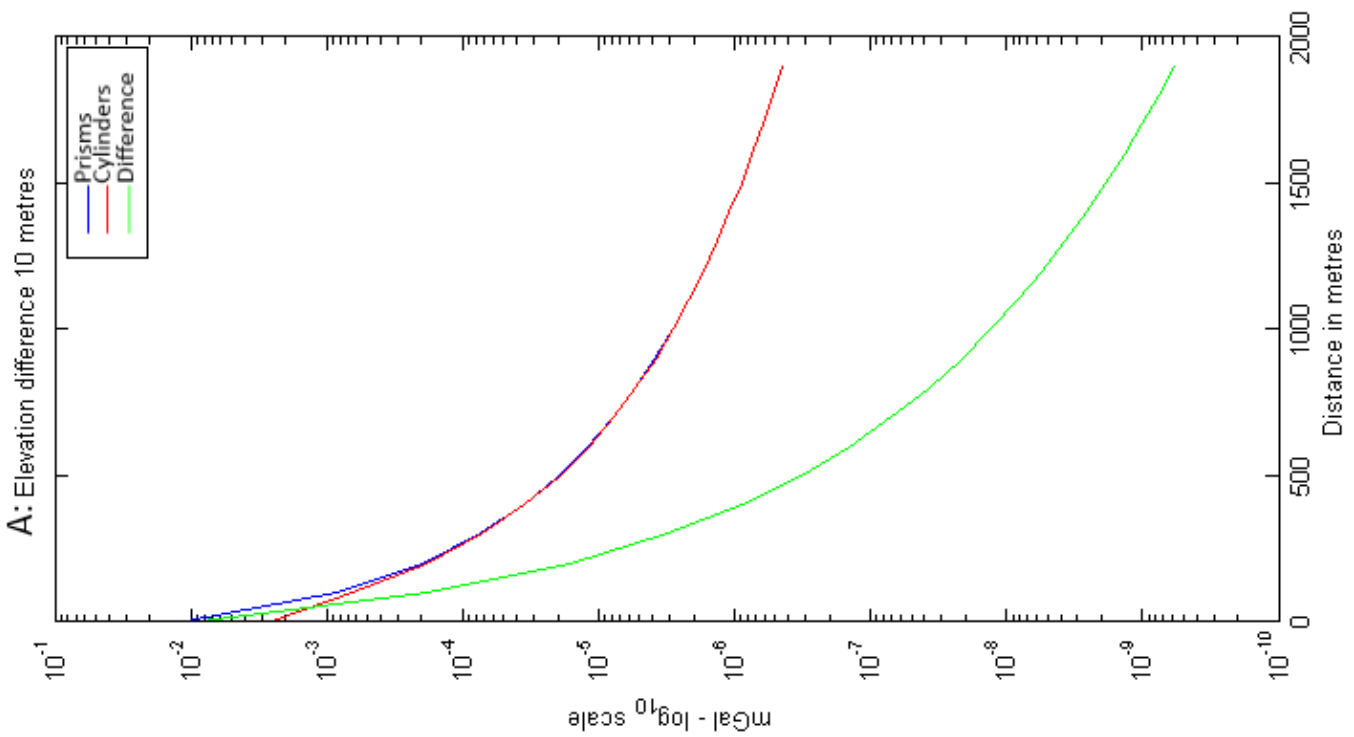

记

屯ै .ี

$\exists$

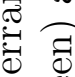

$\pitchfork$

$\stackrel{5}{50} \frac{0}{\sigma^{\circ}}$

$\leftarrow$ ¿

₹

,

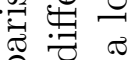

茟

宅

4 ठ

$\ddot{0} \underset{\Theta}{0}$

ง $\tau$

$\stackrel{0}{3}$ ⿹

500

江 毛 
The two methods were also tested at the location of 1000 randomly selected existing gravity sites across New Zealand (described in detail in chapter 4.1). Terrain corrections were calculated for an inner zone (out to $170 \mathrm{~m}$ ) and an outer zone out to $120 \mathrm{~km}$ from an $8 \mathrm{~m}$ digital elevation model. The 1000 randomly selected data points had a good distribution across the country covering areas with both smooth and rough topography as can be seen in figure 2.6.

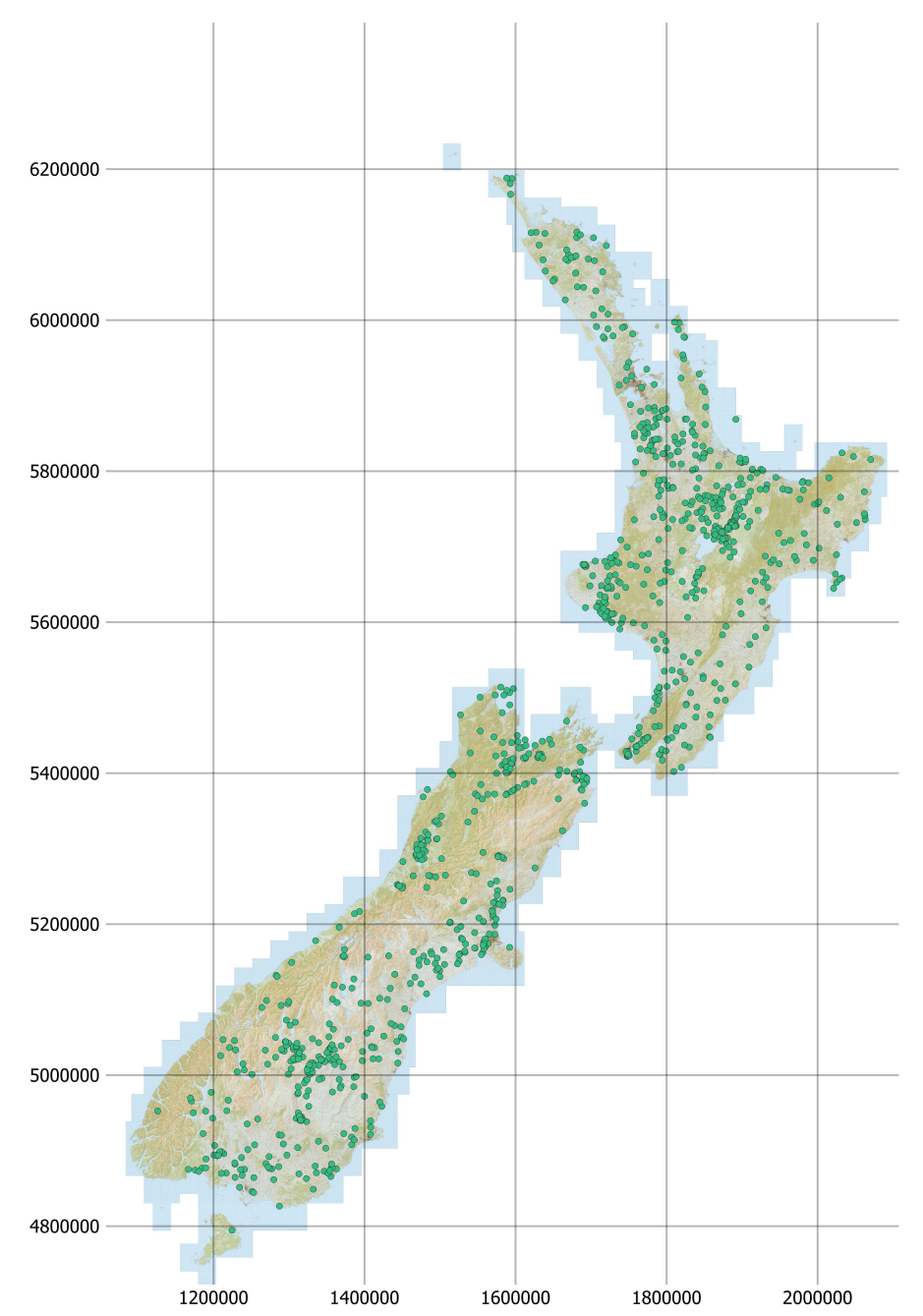

Figure 2.6: A map illustrating the specified locations of the 1000 marks chosen at random from the GNS terrestrial gravity database.

The inner terrain corrections were in good agreement having a difference (Hammer Nagy) with a mean of $0.001 \mathrm{mGal}$ and standard deviation of $0.002 \mathrm{mGal}$ and maximum difference of $0.014 \mathrm{mGal}$. The difference between the two methods for the outer zone terrain correction was similar, with a mean of $-0.003 \mathrm{mGal}$, standard deviation of 0.006 $\mathrm{mGal}$ and maximum difference of $0.023 \mathrm{mGal}$. 


\subsection{Upward and downward continuation of gravity anomalies}

Observed gravity values change with elevation and high frequency gravity signals are attenuated as the observation location moves away from the gravity source. When processing gravity observations made at different elevations (e.g. when combining terrestrial, chapter 5, and airborne gravity data sets, chapter 6) an important step is to ensure they are on a common reference level. This is achieved by upward and downward continuation.

The gravity anomaly $\Delta g$ caused by a sphere of radius $b$ with a density anomaly $\Delta \rho$ (i.e. a density difference between the buried sphere and the surrounding rock) buried at a depth $h$ below the surface is given by the following formula (Telford et al, 1990).

$$
\Delta g=\frac{4 \pi}{3} G \Delta \rho \frac{b^{3} h}{\left(x^{2}+h^{2}\right)^{3 / 2}}
$$

where $\mathrm{x}$ is the Euclidean distance from the centre of the sphere in the horizontal direction and $\mathrm{G}$ is the gravitational constant.

As the depth of the body increases (or equivalently as the gravity field observation point moves away from the observed body in the vertical direction) the gravity anomaly profile across the buried sphere flattens out. This can be seen in the following figure with the illustrative parameter choices $\Delta \rho=0.4\left(\mathrm{Mg} / \mathrm{m}^{3}\right) b=200(\mathrm{~m})$.

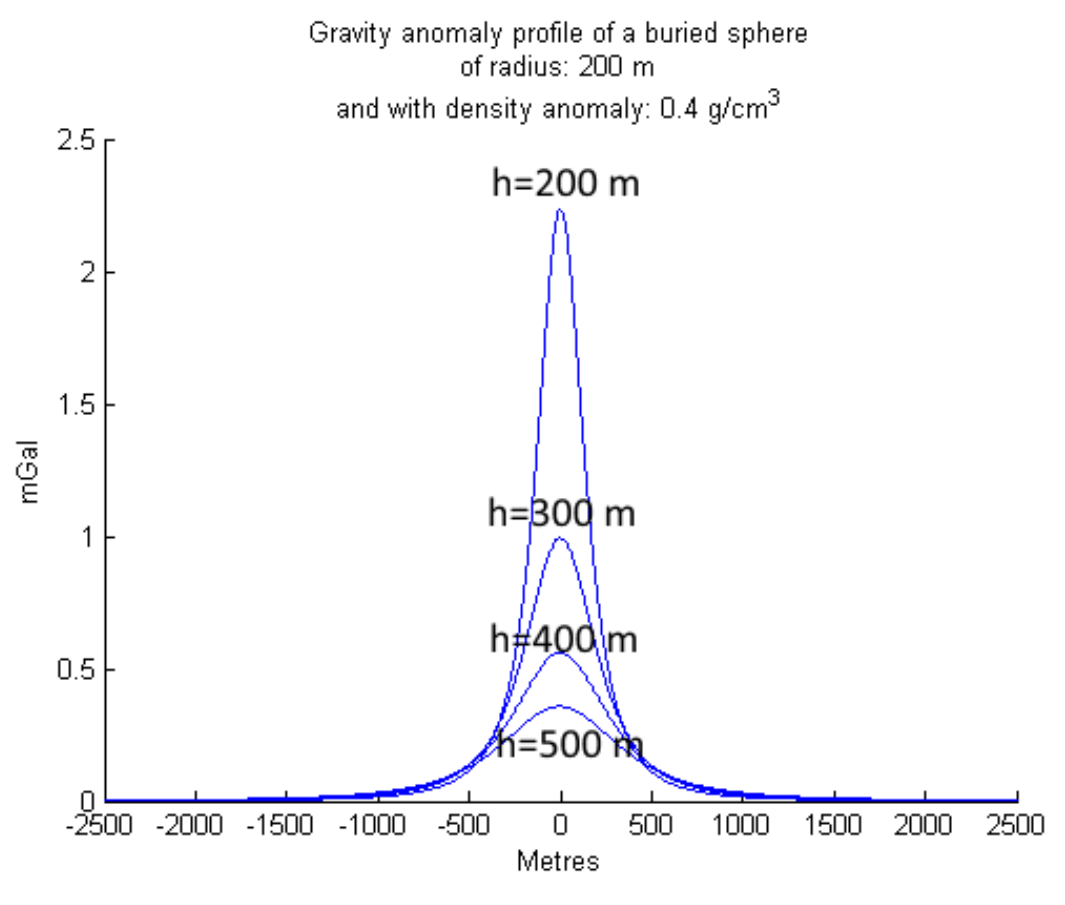

Figure 2.7: The gravity anomaly caused by a buried sphere with a density anomaly of $0.4 \mathrm{Mg} / \mathrm{m}^{3}$, radius of $200 \mathrm{~m}$ and depths varying from 200 to $500 \mathrm{~m}$. 
For a planar approximation, the gravity anomaly $\Delta g(x, y, h)$ at an elevation $h$ is related to the gravity anomaly on a level surface (where $h=0) \Delta g(x, y, 0)$ by the Abel-Poisson integral (Heiskanen and Moritz, 1967),

$$
\Delta g(x, y, h)=\frac{h}{2 \pi} \int_{-\infty}^{\infty} \int_{-\infty}^{\infty} \frac{\Delta g(\alpha, \beta, 0)}{\left[(x-\alpha)^{2}+(y-\beta)^{2}+h^{2}\right]^{3 / 2}} \partial \alpha \partial \beta
$$

This equation can be thought of as representing the gravity field with buried spheres due to the similarity of the integration kernel and equation 2.21 .

\section{The Fourier Domain approach}

Equation 2.22 is a convolution (Schwarz, 1990) and can be expressed as

$$
\Delta g(x, y, h)=\left.w(x, y)\right|_{z=0} ^{h} * \Delta g(\alpha, \beta, 0)
$$

with,

$$
\left.w(x, y)\right|_{z=0} ^{h}=\left.\frac{h}{2 \pi} \frac{1}{\left[(x-\alpha)^{2}+(y-\beta)^{2}+h^{2}\right]^{3 / 2}}\right|_{z=0} ^{h}
$$

In the Fourier domain the convolution corresponds to multiplication. For $\left.W(u, v)\right|_{z=0} ^{h}$ the Fourier transform of $\left.w(x, y)\right|_{z=0} ^{h}$,

$$
\left.W(u, v)\right|_{z=0} ^{h}=e^{-2 \pi h f}
$$

where $f=\sqrt{u^{2}+v^{2}}$. The Fourier transform of $\Delta g(x, y, h)$ given by $\left.G(u, v)\right|_{z=h}$ is then computed from the Fourier transform of $g(x, y, 0)$ given by $\left.G(u, v)\right|_{z=0}$ multiplied by $\left.W(u, v)\right|_{z=0} ^{h}=e^{-2 \pi h f}$ i.e.

$$
\left.G(u, v)\right|_{z=h}=\left.\left.G(u, v)\right|_{z=0} W(u, v)\right|_{z=0} ^{h} .
$$

This operation to find the gravity anomalies at a higher elevation than where they are already known (upward continuation) is essentially a low pass filter i.e. short wavelengths in the gravity anomaly signal are attenuated more than longer wavelengths. If the gravity anomalies are known at an elevation $h_{1}$ and it is required to know them at a lower elevation $h_{2}$ (downward continuation) then,

$$
\left.G(u, v)\right|_{z=h_{2}}=\left.\left.G(u, v)\right|_{z=h_{1}} W(u, v)\right|_{z=h_{1}} ^{z=h_{2}}
$$

or,

$$
\left.G(u, v)\right|_{z=h_{2}}=\left.G(u, v)\right|_{z=h_{1}} e^{2 \pi\left(h_{1}-h_{2}\right) f}
$$

Advantages $\left.G(u, v)\right|_{z=h_{2}}$ is simple to obtain numerically using the fast Fourier transform. The method is quick and easy to implement.

Disadvantages This process amplifies short wavelengths in the gravity signal which can be problematic when the data contain any high frequency noise.

Moreover this approach is problematic since gravity observations are rarely made on a consistently level surface which is a prerequisite to be able to grid them on a plane for the 2D Fourier transform. 


\section{Regularized Least Squares}

Wang et al (2004) construct a system of equations such that the integral equation (2.22) can be solved by a least squares approach i.e. by writing the integral equation (2.22) in matrix form such that

$$
\Delta g(x, y, h)=A \Delta g(\alpha, \beta, 0)
$$

where $A$ is a matrix, with elements determined by the integral kernel, that maps gravity anomalies at one observation level, $h$, to the surface. Observations $\Delta g(x, y, h)$ can then be used to determine gravity anomalies at the surface of the Earth by least squares. The aim of least squares is to minimize the Euclidean norm of the vector $\epsilon$ where,

$$
\epsilon=\Delta g(x, y, h)-A \Delta g(\alpha, \beta, 0)
$$

As with the Fourier domain approach, the downward continuation will amplify high frequency noise. Wang et. al (2004), demonstrate that the amplification of the noise in the signal can be controlled by using Tikhonov regularization (Tikhonov, 1977). This process introduces a matrix $L$ which acts as a differential operator and the aim is to determine $\Delta g(\alpha, \beta, 0)$ subject to minimizing,

$$
\|\epsilon\|^{2}=\|\Delta g(x, y, h)-A \Delta g(\alpha, \beta, 0)\|^{2}+\|\alpha L g(\alpha, \beta, 0)\|^{2}
$$

The use of the matrix $\mathrm{L}$ has a smoothing effect and $\alpha$ is a chosen value which controls the amount of smoothing, acting to partition the signal to noise ratio.

Advantages This method offers an alternative to the Fourier domain approach with the key advantage that the amplification of the noise level in the data can be controlled. Moreover, it allows for gravity observations to be scattered i.e. they are not required to be on a uniform grid, which is more appropriate in practice.

Disadvantages The degree of smoothing determined by $\alpha$ is sensitive, and it is difficult to make a hard and fast choice for this value, and no consideration has been made here for varying noise levels of individual data points. Also, the technique requires large matrices to be constructed and so the method is computationally expensive for large data sets.

\section{Virtual Point Mass Method}

The Rougue theory says that the disturbing potential of the Earth can be approximated by a harmonic function of a sphere located in the Earth called the Bjerhammar spheres (Wang et al, 2004). The virtual point mass method represents sources of the Earth gravity field as various Bjerhammar sphere where the potential and derivatives match gravity observations to some precision. Gravity anomalies, $\Delta g_{i}, i=1,2,3, \ldots n$, can be written by the method as,

$$
\Delta g_{i}=G\left(\sum_{j=1}^{m} \frac{r_{i}-R_{j}^{B} \cos \left(\psi_{i, j}\right)}{\rho_{i, j}^{3}}-\frac{2}{\rho_{i, j} r_{i}}\right) M_{j}
$$

here, $r_{i}$ is the geocentric range of the $i_{t} h$ gravity anomaly, $R_{j}^{B}=R-D_{j}$ is the radius of the Bjerhammar sphere, $D_{j}$ is the depth of the $j_{t} h$ point mass and $\rho_{i, j}$ and $\psi_{i, j}$ are the 
distance and spherical angle between the gravity anomaly observations $i$ and $j$. R is the mean Earth radius in the region,

$$
R=\frac{1}{n} \sum_{i=1}^{n}\left(R_{i}+H_{i}\right)
$$

where $H_{i}$ is the elevation of each gravity observation.

Equation 2.32 can be written in matrix form such that,

$$
\Delta g=A M
$$

Here, $\mathrm{M}$ is a vector of the $m$ point masses and $A$ is an $n \times m$ vector of coefficients. M can be solved for by least squares as,

$$
M=\left(A^{T} P A\right)^{-1} A^{T} P \Delta g
$$

where $P$ is a weight matrix of the observations $\Delta g$.

Using equation 2.32 a new matrix can then be constructed $A^{\prime}$, which corresponds to the relationship between the point masses $M$ and the gravity anomalies, $\Delta g_{h=0}$ at the surface of the Earth. $\Delta g_{h=} 0$ can then by computed by the following,

$$
\Delta g_{h=0}=A^{\prime} M
$$

Advantages As with the regularized least squares approach, the observation noise is not heavily amplified by the downward continuation procedure. This is because the highest frequency in the resulting gravity anomalies at the surface of the earth is related to the number of point masses $n$ which are determined by the procedure.

Using a weight matrix $P$ the accuracy of each individual gravity anomaly can be taken into account.

Disadvantages Equation 2.32 makes an assumption that the height of the gravity anomaly observations is consistent. This is not practical in the general case with actual field observations.

\section{Spherical Interior Dirchlet's Harmonic Solution Method}

Following Wang et al (2004), let $V\left(R^{\prime}, \gamma^{\prime}, \lambda^{\prime}\right)$ be a harmonic function that is known on a spherical surface $\Sigma^{\prime}$. Let $\Sigma$ be a spherical surface inside of $\Sigma^{\prime}$. The harmonic function on the surface of $\Sigma, V(R, \gamma, \lambda)$ is given by the following integral of $V$ on $\Sigma^{\prime}$ (Shi, 1999).

$$
V(R, \gamma, \lambda)=\frac{R^{\prime}\left(R^{\prime 2}-R_{Q}^{2}\right)}{4 \pi} \iint_{\Sigma^{\prime}} \frac{V\left(R^{\prime}, \gamma^{\prime}, \lambda^{\prime}\right)}{\rho^{3}} d \sigma
$$

Here, $R_{Q}$ is the radius of the inner sphere $\Sigma$ and $\rho$ is the distance between the moving point of the integral and integral evaluation point. For gravity anomaly observations $\Delta g$ 
known on the surface of $\Sigma$, the function given by $R_{Q} \Delta g$ is harmonic. Then in terms of equation 2.22 , if gravity anomaly observations are known on the surface of $\Sigma^{\prime}$, they can be calculated on the surface of $\Sigma$ by evaluating the following integral,

$$
\Delta g(R, \gamma, \lambda)=\frac{R^{\prime}\left(R^{\prime 2}-R_{Q}^{2}\right)}{4 \pi R_{Q}} \iint_{\Sigma^{\prime}} \frac{\Delta g^{\prime}\left(R^{\prime}, \gamma^{\prime}, \lambda^{\prime}\right)}{\rho^{3}} d \sigma
$$

Advantages This method avoids solving equation 2.22, and the gravity anomalies are directly determined by evaluating the integral.

Disadvantages The approach assumes again that the gravity anomaly observations are available at a consistent height and continuously at discrete intervals so that the integral can be evaluated. This is not possible with scattered observation data points which is the typical case. Additonally, the method does not account for observation error, which will propagate through the integral.

\section{Least squares collocation method}

In general least squares collocation is a sophisticated interpolation method. An $n \times 1$ vector of interpolated values $s$ is interpolated from an $m \times 1$ vector observed quantities $g$ using the fundamental equation of least squares collocation (as given by Moritz (1972)) by,

$$
\hat{s}=C_{s g}\left(C_{g g}+N\right)^{-1} x
$$

Here,

- $C_{s g}$ is the $n \times m$ covariance matrix of the desired signal $s$ and observations $g$

- $C_{g g}$ is the $m \times m$ covariance matrix of the observations and

- $N$ is the covariance matrix of the measurement noise.

The element in row $i=1,2,3, \ldots, n$ and $j=1,2,3, \ldots, m$ of the matrix $C_{s g}$ is determined by a function $f$ which is dependent on the the position of the interpolation point $s_{i}\left(x_{i}, y_{i}\right)$ and the position of observation point of $g_{j}\left(x_{j}, y_{j}\right)$. i.e.

$$
\left(C_{s g}\right)_{i, j}=f\left(x_{i}, y_{i}, x_{j}, y_{j}\right)
$$

and elements $i=12,3, \ldots, m$ and $j=, 12,3, \ldots, m$ of $C_{g g}$ are determined by the same function, but the from position of pairs of the observations $g\left(x_{i}, y_{i}\right)$ and $g\left(x_{j}, y_{j}\right)$

$$
\left(C_{g g}\right)_{i, j}=f\left(x_{i}, y_{i}, x_{j}, y_{j}\right)
$$

The function $f$ must be a positive definite function to ensure the matrices can be inverted, for example the Gaussian function given by,

$$
F\left(x_{i}, y_{i}, x_{j}, y_{j}\right)=C_{0} e^{-\alpha^{2} r^{2}}
$$




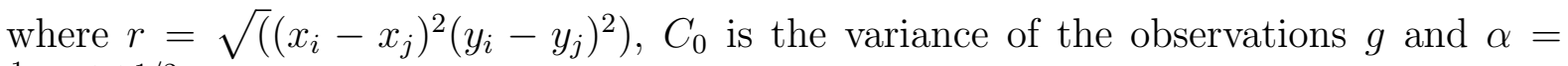
$\frac{1}{d} \log (2)^{1 / 2}$ were $d$ is the value at which the spatial correlation of the data reduces to half, (a value typically referred to as the correlation length). The noise matrix $N$ acts to regularize the interpolated value $s$ by attributing part of the observation signal to noise.

This method can be used to interpolate gravity anomaly quantities with the choice of an appropriate (harmonic) covariance function F. Forsberg (1987) gives an appropriate covariance function to interpolate gravity data that additionally acts three dimensionally i.e. $F=F\left(x_{i}, y_{i}, z_{i}, x_{j}, y_{j}, z_{j}\right)$.

The covariance function $F$ given by Forsberg (1987) is,

$$
F\left(x_{i}, y_{i}, z_{i}, x_{j}, y_{j}, z_{j}\right)=-f \sum_{k} \alpha_{k} \log \left(D_{k}+\sqrt{\left(x_{i}-x_{j}\right)^{2}+\left(y_{i}-y_{j}\right)^{2}+\left(D_{k}+z_{i}+z_{j}\right)^{2}}\right) .
$$

with $\alpha_{k}=1,-3,3,-1, D_{k}=D+k T$ and,

$$
f=C_{0} \log \left(\frac{(D+T)^{3}(D+3 T)}{D(D+2 T)^{3}}\right) .
$$

The values $C_{0}, D$ and $T$ are fit to the observation data empirical spatial covariance values, where $C_{0}$ is the observation variance, $D$ is the depth to the Bjerhammar sphere (as discussed in the point mass downward continuation approach) and $T$ acts to suppress long wavelengths which are not predictable from observation data over a small region.

Forsberg (2003) suggests the following parameters for gridding gravity anomaly values; $C_{0}=100 \mathrm{mGal}^{2}, D=10 \mathrm{~km}$ and $T=50 \mathrm{~km}$, Toth (2009) uses parameter values of $C_{0}=57.7 \mathrm{mGal}^{2} D=5 \mathrm{~km}$ and $T=9 \mathrm{~km}$ when dealing with a very high frequency gravity signal and optimal fit for some airborne data in this thesis is obtained with $C_{0}=75.8 \mathrm{mGal}^{2}, D=9.5 \mathrm{~km}$ and $T=48.5 \mathrm{~km}$ in chapter 6 which is remarkably close to the Forsberg (2003) suggested parameters.

Advantages This method allows for the gravity signal to be interpolated anywhere in three dimensional space, and avoids having to solve equation 2.22.

It does not require the observation data to be available at consistent grid intervals (i.e. scatter data points can be used) and it does not require the data to be available at a consistent elevation.

Due to the inclusion of the matrix $N$ which allows an approximate noise level to be assigned to each observation, the method is particularly powerful when combining various data sets of different quality onto a single grid at any desired elevation (e.g. on the surface of the Earth).

Disadvantages The method requires that a large matrix is inverted which for $m$ observation points, has $m^{2}$ elements. This is problematic for large data sets. However it is possible to consider the data in small regions to break the problem down into manageable chunks. 
Summary Least squares collocation has been chosen as the method to downward continue and combine datasets in this thesis. It is preferable over other methods discussed here since it allows for varying elevation of observation heights (which is important for the airborne data discussed in chapter 6) and allows for an optimal combination of data sets with different qualities (which is important for combining airborne and terrestrial measurements in the New Zealand setting as discussed in chapter 7).

The method of least squares collocation is discussed in more detail in chapter 7 and is used to combine several different types of gravity observations which were made at varying elevations on to a single grid at the topographic surface. The methodology is also used extensively in section 6.1 to downward continue some airborne gravity observations for a comparison with ground based gravity data. 


\subsection{Earth tides}

The tidal effect of the sun and moon is periodic over time but is not zero on average, so it can be thought of as having periodic (which is zero on average) and permanent components. The permanent tide is low at the poles and high in the equatorial areas (Ekman, 1989).

There are three commonly used models to represent tides which are as follows;

- Tide-free: this model eliminates the permanent tidal deformation of the shape of the Earth and the tidal potential gravity field. i.e. The tidal effects of the sun and moon are removed from the potential field and shape of the Earth, which corresponds to physically moving the sun and moon out to infinity.

- Mean-tide: this model retains the permanent deformation of the shape of the Earth and the potential field due to tidal forcing.

- Zero-tide: this model retains the permanent deformation of the shape of the Earth under tidal forcing but removes the effect on the potential field.

The International Gravity Standardization Net 1971 (ISGN71 see: http://www.dtic.mil/cgibin/GetTRDoc?AD=ADA006203) gravity system is in terms of the mean-tide (ISGN71 is an absolute datum for worldwide gravity networks) and gravity observations made in New Zealand have been put in terms of this standard (Stagpoole, 2012).

Resolution 16 of the International Association of Geodesy (IAG) in 1983 approved the zero-tide model as the preferred model for Geoid modeling (Amos, 2007). This is because it is compliant with Stokes' formula since it assumed all external masses are removed (unlike the mean-tide model) and unlike the tide-free model does not require the inclusion of Love numbers to represent the rigidity of the Earth in response to tidal forces.

Ekman (1989) gives the following conversions for height differences (between one orthometric height observation and another), $\Delta H$ (in $\mathrm{cm}$ ), between each of the tidal models (with subscripts $n, m, z$ denoting the tide-free, mean-tide and zero-tide models) as,

$$
\begin{gathered}
\Delta H_{m}=\Delta H_{z}+29.6\left(\sin ^{2}\left(\phi_{N}\right)-\sin ^{2}\left(\phi_{S}\right)\right) \\
\Delta H_{z}=\Delta H_{n}+(\gamma-1) 29.6\left(\sin ^{2}\left(\phi_{N}\right)-\sin ^{2}\left(\phi_{S}\right)\right) \\
\Delta H_{m}=\Delta H_{n}+\gamma 29.6\left(\sin ^{2}\left(\phi_{N}\right)-\sin ^{2}\left(\phi_{S}\right)\right)
\end{gathered}
$$

Where $\phi_{N}$ and $\phi_{S}$ are the latitude of the northern height observation and southern height observation respectively and $\gamma$ is the normal gravity. Similarly, for gravity anomalies $\Delta g$ ( $\mathrm{mGal})$, the conversions between tidal systems are given as.

$$
\begin{gathered}
\Delta g_{m}=\Delta g_{z}-3.04+9.12 \sin ^{2}(\phi) \\
\Delta g_{z}=\Delta g_{n}+(\delta-1)\left(-3.04+9.12 \sin ^{2}(\phi)\right) \\
\Delta g_{m}=\Delta g_{n}+(\delta)\left(-3.04+9.12 \sin ^{2}(\phi)\right)
\end{gathered}
$$

where $\delta$ is a theoretical constant value $\approx 1.53$ and $\phi$ is latitude. 


\subsection{Geoid undulations determined by Stokes' inte- gral}

Following Heiskanen and Moritz (1967), Stokes' integral gives the geoid undulation $N$ at a point $\left(\theta_{p}, \lambda_{p}\right)$ as,

$$
N\left(\theta_{p}, \lambda_{p}\right)=\frac{R}{4 \pi \gamma} \int_{\sigma} \Delta g(\theta, \lambda) S(\psi) \partial \sigma
$$

where,

- $R$ : average Earth radius,

- $N$ : geoid undulation,

- $\gamma$ : normal (ellipsoidal) gravity (at $\left.\theta_{p}\right)$,

- $\Delta g$ gravity anomaly,

- $\partial \sigma$ : infinitesimal surface element

- $S(\psi)$ : Stokes' kernel function.

Stokes' kernel function has the form,

$$
S(\psi)=\frac{1}{s}-4-6 s+10 s^{2}-\left(3-6 s^{2}\right) \ln \left(s+s^{2}\right)
$$

where,

$$
s=\sin ^{2} \frac{\psi}{2}=\sin ^{2}\left(\frac{\theta_{p}-\theta}{2}\right)+\sin ^{2}\left(\frac{\lambda_{p}-\lambda}{2}\right) \cos \left(\theta_{p}\right) \cos (\theta)
$$

Moreover, $S(\psi)$ can also be expressed as an infinite Fourier series of Legendre polynomials given by,

$$
S(\psi)=\sum_{n=2}^{n=\infty} \frac{2 n+1}{n-1} P_{n}(\psi) .
$$

The solution, $\mathrm{N}$, to equation 2.45 has zero coefficients for the spherical harmonic terms of degree one and zero and in practice they are provided by a global gravity model.

The solution to this equation relies on,

1. $\Delta g$ being reduced to the surface of the geoid,

2. there being no topographical masses outside of the geoid and

3. $\Delta g$ being available continuously over the surface of the Earth.

Points 1 and 2 can be resolved using Helmert's second method of condensation and point 3 can be dealt with by using the remove-compute-restore technique. These methods are described in the subsequent sections. 


\subsubsection{Helmert's second method of condensation:}

Helmert's second method of condensation resolves points 1 and 2 by implementing the following 5 steps (Amos, 2007).

1. Direct topographical effect: Condense the gravitational effect of topography to the surface of the Geoid.

2. Downward continuation: Downward continue the topography reduced gravity anomalies to the surface of the geoid by solving equation 2.22 .

3. Compute the Co-geoid: Use Stokes' integral (equation 2.45) with the downward continued reduced gravity anomalies to evaluate a surface known as the Co-geoid.

4. Primary indirect topographical effect: Perform a reverse correction to compensate for the topographic reduction of step 1.

5. Secondary indirect topographical effect: Perform a correction to account for the difference between the height of the true geoid and the co-geoid. This step is typically ignored as it is of the order of $1 \mathrm{~mm}$ (Vanicek, 1987).

Direct topographical effect and Downward continuation: In practice the first two steps can be performed as follows,

1. From observed scattered absolute gravity values, calculate scattered Bouguer anomalies by subtracting the normal gravity (following section 2.2.1), the free air effect (following section 2.2.2) and the Bouguer slab and then add back the terrain correction (following section 2.2.3).

2. The Bouguer anomaly data are then downward continued to the surface of the geoid by solving equation 2.22. However this step is often disregarded when the Bouguer anomalies are available at the topographic surface. This is because the distance to downward continue is reasonably small and the Bouguer gravity anomaly signal is fairly long wavelength so the effect of the downward continuation is negligible.

3. Calculate Bouguer anomalies at regular grid points (using some interpolation algorithm) from the scattered data points.

4. Apply a reverse Bouguer slab correction by adding $0.0419 \rho H^{\text {orth }}$ to the gridded Bouguer gravity anomalies.

This process overall returns gridded Faye anomalies; these are in effect terrain corrected free air anomalies. The reverse Bouguer slab correction can be performed by extracting heights from a digital elevation model (DEM). By gridding the Bouguer anomalies prior to the reverse Bouguer slab correction (i.e. performing step 3 before step 4 rather than vice versa), the potential effect of topographic aliasing in the gridded signal is reduced (Featherstone and Kirby, 2000), since the topographic effect is high frequency and sparse spatial sampling will result in aliasing. 
Primary indirect topographical effect The primary indirect topographical effect is given by Wichiencharoen (1982) as,

$$
N_{\text {ind }}(P)=\frac{-\pi G \rho H_{P}^{2}}{\gamma}-\frac{G \rho R^{2}}{6 \gamma} \int_{\sigma} \frac{H^{3}-H_{p}^{3}}{l^{3}} d \sigma .
$$

Here $H_{P}$ is the orthometric height of the point $P$ and $l$ is the distance between $P$ and the moving point of the integration. When equation 2.48 is written as a Taylor series and evaluated with a dense DEM it becomes quickly divergent after the quadratic term. For this reason in practical use it is only evaluated using the first term of the equation above (Amos, 2007).

\subsubsection{Remove - Compute - Restore (RCR):}

To compute $N$ using equation 2.31 requires that the gravity data are available continuously over the surface of the Earth; however this is not practical. This issue is resolved by removing a long wavelength gravity anomaly from the gridded Faye anomaly observations (Remove), computing a residual geoid undulation using Stokes' integral formula with a spherical integration cap and a modified kernel (Compute) then adding back a long wavelength geoid undulation (Restore) (Hirt, 2012). i.e.

1. Calculate the long wavelength anomaly $\Delta g_{L}$ using a global gravity model (see section 5.1) up to some maximum degree and order $L$ at each of the gridded measurement locations.

2. Calculate a residual gravity anomaly signal by removing long wavelength gravity anomalies from gridded Faye anomalies, $\Delta g_{\text {res }}=\Delta g-\Delta g_{L}$.

3. Use the Stokes' integral formula with a spherical integration cap $\psi_{0}$ and a modified kernel $S^{*}(\psi)$ to compute the residual geoid undulation $N_{\text {res }}$. i.e.

$$
N_{\text {res }}\left(\theta_{p}, \lambda_{p}\right)=\frac{R}{4 \pi \gamma} \int_{\psi_{0}} \Delta g_{r e s}(\theta, \lambda) S^{*}(\psi) \partial \sigma
$$

4. Calculate the full geoid undulation $N$ by adding back the long wavelength geoid undulation, $N=N_{L}+N_{\text {res }}$. $N_{L}$ is evaluated from the same global gravity model (see section 5.1) as in step 1. i.e.

$$
N\left(\theta_{p}, \lambda_{p}\right)=N_{L}\left(\theta_{p}, \lambda_{p}\right)+\frac{R}{4 \pi \gamma} \int_{\psi_{0}} \Delta g_{r e s}(\theta, \lambda) S^{*}(\psi) \partial \sigma
$$

\subsubsection{Stokes' kernel modification:}

During the RCR process, described in section 2.5.2, long wavelengths have been removed from the gridded gravity anomalies, and so Stokes' integration need only be performed on a limited integration radius denoted $\psi_{0}$. However, this requires the use of a modified kernel to avoid the truncation error incurred due to omission of data outside of the integration radius. Heiskanen and Moritz (1967) gives the truncation error, $\delta N$ as follows,

$$
N=\frac{R}{4 \pi \gamma} \int_{0}^{\psi_{0}} \int_{0}^{2 \pi} \Delta g(\theta, \lambda) S(\psi) \sin (\psi) d \psi d \alpha+\delta N
$$


where

$$
\delta N=\frac{R}{4 \pi \gamma} \int_{\psi_{0}}^{\pi} \int_{0}^{2 \pi} \Delta g(\theta, \lambda) K(\psi) \sin (\psi) d \psi d \alpha
$$

with the kernel $K$ given by,

$$
\begin{array}{ll}
K(\psi)=0 & \text { for } 0 \leq \psi<\psi_{0} \\
K(\psi)=S(\psi) & \text { for } \psi_{0} \leq \psi \leq \pi
\end{array}
$$

By writing $K$ as a series of Legendre polynomials equation 2.52 can be expressed as the following series expansion,

$$
\delta N=\frac{R}{2 \gamma} \sum_{n}^{\infty} \Delta g(\theta, \lambda) Q_{n}\left(\psi_{0}\right)
$$

with

$$
Q_{n}(\psi)=\int_{\psi_{0}}^{\pi} S(\psi) P_{n}(\psi) \sin (\psi) d \psi
$$

where $P_{n}$ are Legendre polynomials. The coefficients $Q_{n}\left(\psi_{0}\right)$ govern the rate at which the truncation error associated with $S(\psi)$ converges and the rate is determined by the smoothness of the error kernel $K(\psi)$.

Meissl modified Stokes' Kernel, $S_{M}(\psi)$ : In equation 2.53, $K(\psi)$ is not continuous at $\psi=\psi_{0}$. Meissl (1971) makes $K(\psi)$ continuous by subtracting $S\left(\psi_{0}\right)$ from the kernel. i.e.

$$
\begin{array}{ll}
S_{M}(\psi)=S(\psi)-S\left(\psi_{0}\right) & \text { for } 0 \leq \psi<\psi_{0} \\
S_{M}(\psi)=0 & \text { for } \psi_{0} \leq \psi \leq \pi
\end{array}
$$

and the error kernel is then given by,

$$
\begin{array}{ll}
K_{M}(\psi)=S\left(\psi_{0}\right) & \text { for } 0 \leq \psi<\psi_{0} \\
K_{M}(\psi)=S(\psi) & \text { for } \psi_{0} \leq \psi \leq \pi
\end{array}
$$

which is continuous for all $\psi$, and since $K_{M}(\psi)$ is smoother than $K(\psi)$ the convergence of the of the truncation error improves.

Wong and Gore modified Stokes' Kernel, $S_{W G}(\psi)$ : The Wong and Gore (1969) modified Stokes' kernel takes a different approach to Meissl, by subtracting low degree Legendre polynomial terms up to some maximum $p$ from equation 2.47. i.e.

$$
S_{W G}(\psi)=S(\psi)-\sum_{n=2}^{n=p} \frac{2 n+1}{n-1} P_{n}(\psi) .
$$

Here $p$ is considered to be strictly less than the maximum degree and order of the global gravity model removed in step 2 of section 2.5.2. This time the error kernel $K(\psi)$ is given by,

$$
\begin{array}{ll}
K_{W G}(\psi)=0 & \text { for } 0 \leq \psi<\psi_{0} \\
K_{W G}(\psi)=S_{W G}(\psi) & \text { for } \psi_{0} \leq \psi \leq \pi
\end{array}
$$


which is discontinuous at $\psi_{0}$ but converges quicker than $K(\psi)$ outside the integration cap due to the removal of lower order terms in the series expansion of $S(\psi)$. i.e. this modification reduces low frequency errors in $N$ due to the integration cap since the low order terms of the integration kernel $S(\psi)$ are not used. This modification is essentially a high pass filter.

Vanicek and Kleusberg modified Stokes' Kernel, $S_{V K}(\psi)$ : The Vanicek and Kleusberg (1987) modified Stokes' kernel is similar to the Wong and Gore (1969) modified Stokes' kernel with an additional term. It is given by the following,

$$
S_{V K}(\psi)=S(\psi)-\sum_{n=2}^{n=p} \frac{2 n+1}{n-1} P_{n}(\psi)-\sum_{k=2}^{k=L} \frac{2 k+1}{2} t_{k}\left(\psi_{0}\right) P_{k}(\psi) .
$$

Here, $t_{k}\left(\psi_{0}\right)$ are coefficients which are determined by finding the least squares solution to the following set of the linear equations

$$
\sum_{k=2}^{k=L} \frac{2 k+1}{2} t_{k}\left(\psi_{0}\right) e_{n k}(\psi)=Q_{n}\left(\psi_{0}\right)-\sum_{k=2}^{k=L} \frac{2 k+1}{2} e_{n k}(\psi)
$$

for $n=2,3,4,5, \ldots, L . Q_{n}\left(\psi_{0}\right)$ are given by equation 2.55 and $e_{n k}(\psi)$ are given by,

$$
e_{n k}(\psi)=\int_{\psi_{0}}^{\pi} P_{n}(\psi) P_{k}(\psi) \sin (\psi) d \psi
$$

This solution has the effect of minimizing the $L_{2}$ norm of the error kernel $K_{V K}(\psi)$ with respect to the integration cap $\psi_{0}$, so this modified Stokes' integration kernel is advantageous over the Wong and Gore modified kernel since the error kernel is smaller. The error kernel here is given by,

$$
\begin{array}{lr}
K_{V K}(\psi)=0 & \text { for } 0 \leq \psi<\psi_{0} \\
K_{V K}(\psi)=S_{V K}(\psi) & \text { for } \psi_{0} \leq \psi \leq \pi .
\end{array}
$$

Heck and Gruninger modified Stokes' Kernel, $S_{H G}(\psi)$ : The Heck and Gruninger(1987) modified Stokes' kernel is given by the following,

$$
\begin{array}{ll}
S_{H G}(\psi)=S_{W G}(\psi)-S_{W G}\left(\psi_{0}\right) & \text { for } 0 \leq \psi<\psi_{0} \\
S_{H G}(\psi)=0 & \text { for } \psi_{0} \leq \psi \leq \pi
\end{array}
$$

It essentially applies a similar modification to the Wong and Gore modified Stokes' kernel as Meissl applied to the unmodified kernel. This makes the Wong and Gore kernel continuous at the boundary of the spherical cap and so improves the smoothness of the error kernel which improves the truncation convergence rate. The error kernel is given by the following,

$$
\begin{array}{ll}
K_{H G}(\psi)=S_{W G}\left(\psi_{0}\right) & \text { for } 0 \leq \psi<\psi_{0} \\
K_{H G}(\psi)=S_{W G}(\psi) & \text { for } \psi_{0} \leq \psi \leq \pi
\end{array}
$$


Featherstone et al. modified Stokes' Kernel (1998), $S_{F}(\psi)$ : The Featherstone et al. (1998) modified Stokes' kernel is given by the following,

$$
\begin{array}{lr}
S_{F}(\psi)=S_{V K}(\psi)-S_{V K}\left(\psi_{0}\right) & \text { for } 0 \leq \psi<\psi_{0} \\
S_{F}(\psi)=0 & \text { for } \psi_{0} \leq \psi \leq \pi .
\end{array}
$$

and the error kernel is given by

$$
\begin{array}{ll}
K_{H G}(\psi)=S_{F}\left(\psi_{0}\right) & \text { for } 0 \leq \psi<\psi_{0} \\
K_{H G}(\psi)=S_{F}(\psi) & \text { for } \psi_{0} \leq \psi \leq \pi
\end{array}
$$

This kernel modification incorporates the advantages of all others. It is continuous on the boundary of the spherical cap and also incorporates the smaller error kernel values resulting of the least squares fit of the linear equations given by equation 2.61.

In theory this kernel is then optimum for use in regional geoid height determination, however some instabilities have been noted by Amos (2007) when $L$ is taken to be large and $\psi_{0}$ small (for example $L=100$ and $\psi_{0}=0.5$ ). However, as noted by Featherstone et al (2001), these parameter combinations are unlikely when reliable long wavelength gravity anomalies are provided (from a global gravity model) for the remove stage of the processing.

\subsection{Summary}

In this section the notation for the disturbing potential $T$, geoid undulation $N$ and gravity anomalies $\Delta g$ were given. Bruns' equation and the geodetic boundary value problem were defined which are the crucial equations in Geoid modeling.

Upward and downward continuation of gravity anomalies/disturbances was defined and the main methods have been given. Least squares collocation is the preferred method for downward continuation since it appropriately deals with data at various initial heights and the high frequency noise amplification can be controlled by the regularization effects of the noise matrix assigned to the observations.

The concept of permanent Earth tides has been defined and transformations between different tidal models are given. Resolution 16 of the International Association of Geodesy (IAG) in 1983 approved the zero-tide model as the preferred model for Geoid modeling.

The solution to the geodetic boundary value problem by Stokes' integral was given. Several practical considerations must be made for computing regional quasi-geoid, namely $\Delta g$ being on the surface of the geoid, there being no topographical masses outside of the geoid and $\Delta g$ being available continuously over the surface of the Earth. The processes to deal with these considerations has been discussed. 


\section{Chapter 3}

\section{Vertical Datums in New Zealand}

\subsection{What is a vertical datum?}

A vertical datum has two components, a specified height system and a defined reference surface. Most vertical datums which are used are gravimetric, i.e. physical heights that are based on the geoid/quasigeoid, or geodetic, i.e. ellipsoidal heights above on some reference ellipsoid.

The type of height system which is chosen is dependent on the type of observations which are made (Amos, 2007). For example, if height observations are made without gravity measurements then only ellipsoidal heights and normal-orthometric heights can be used. As demonstrated in Chapter 1, normal-orthometric heights can be calculated using just the normal gravity field and the quasigeoid separation from the ellipsoid, whereas orthometric heights are dependent on determining the gravity potential at the observation location to compute the geopotential number $C$.

\subsection{New Zealand's Local Vertical Datums}

In New Zealand normal-orthometric heights are normally used. Traditionally, height transfers have been performed using precise leveling, which has an estimated accuracy of $\pm 2 \mathrm{~mm} \sqrt{k}$ where $k$ is the distance the height has been transferfed in $\mathrm{km}$ (Amos, 2007). Precise leveling gives a series of relative measurements in terms of some reference point where the normal-orthometric height is known. When the leveling observations are coupled with GPS measurements, height anomalies (i.e. quasigeoid separation from the ellipsoid) can be calculated directly.

The primary precise leveling network in New Zealand contains in excess of 16,000 km of measurements (Amos, 2007). The leveling loops are tied off to one of 13 mean sea level (MSL) estimations from tide gauge data. The mean sea level estimated for each of the 13 locations is complicated by long term effects due to rise in mean sea level and short term effects such as tidal effects of the sun and moon (which requires 18.6 years of hourly measurements to accurately predict) and sea surface topography (e.g. due to salinity or prevailing winds). 
The rise in mean sea level in New Zealand is well documented, originally reported by Hannah (1990) and its implications in Hannah (1989) with updated results in Hannah (2004), Hannah et al (2010) and Hannah and Bell (2012). Initially tide gauge data from 4 ports was considered (Auckland, Wellington, Lyttelton and Dunedin) where the measurements span 70-100 years. A long term linear trend of $1.7 \pm 0.1 \mathrm{~mm}$ can be seen in the tide gauge data which is attributable to a rise in sea level. This is shown in figure 3.2 of the annual mean sea level estimate from tide gauge data at these four ports over a time period approximately spanning the last 100 years.

The mean sea level the leveling data are reference to will be fixed at some epoch, this will result in a difference between the leveling heights and true mean sea level since it is time dependent. Comparing leveling heights referenced to historic mean sea level measurements will always be negatively offset from the true mean sea level due to sea level rise. Moreover, MSL for other local vertical datums (LVD), (i.e. those other than Auckland, Wellington, Lyttelton and Dunedin) has typically been measured over substantially shorter time periods, some only over a period of 3 years. This means the contribution of the short term tidal effects cannot be estimated accurately and sea surface variability is difficult to predict, moreover these data are unreliable for long term mean sea level rise estimations.

For these reasons the 13 MSL estimations are offset from true mean sea level by some constant value due to fixing the mean sea level at some epoch and moreover the short term effects mean that individual mean sea level estimations are not on the same equipotential surface. This results in 13 separate LVD which are not directly comparable. However, Amos (2007) specifies that a simple transformation from one LVD to any other and true mean sea level can be defined in terms of a constant offset value (Amos, 2007). This means a set of normal orthometric heights defined in terms of one LVD can be transformed to normal-orthometric heights in terms any other by adding a constant value. 


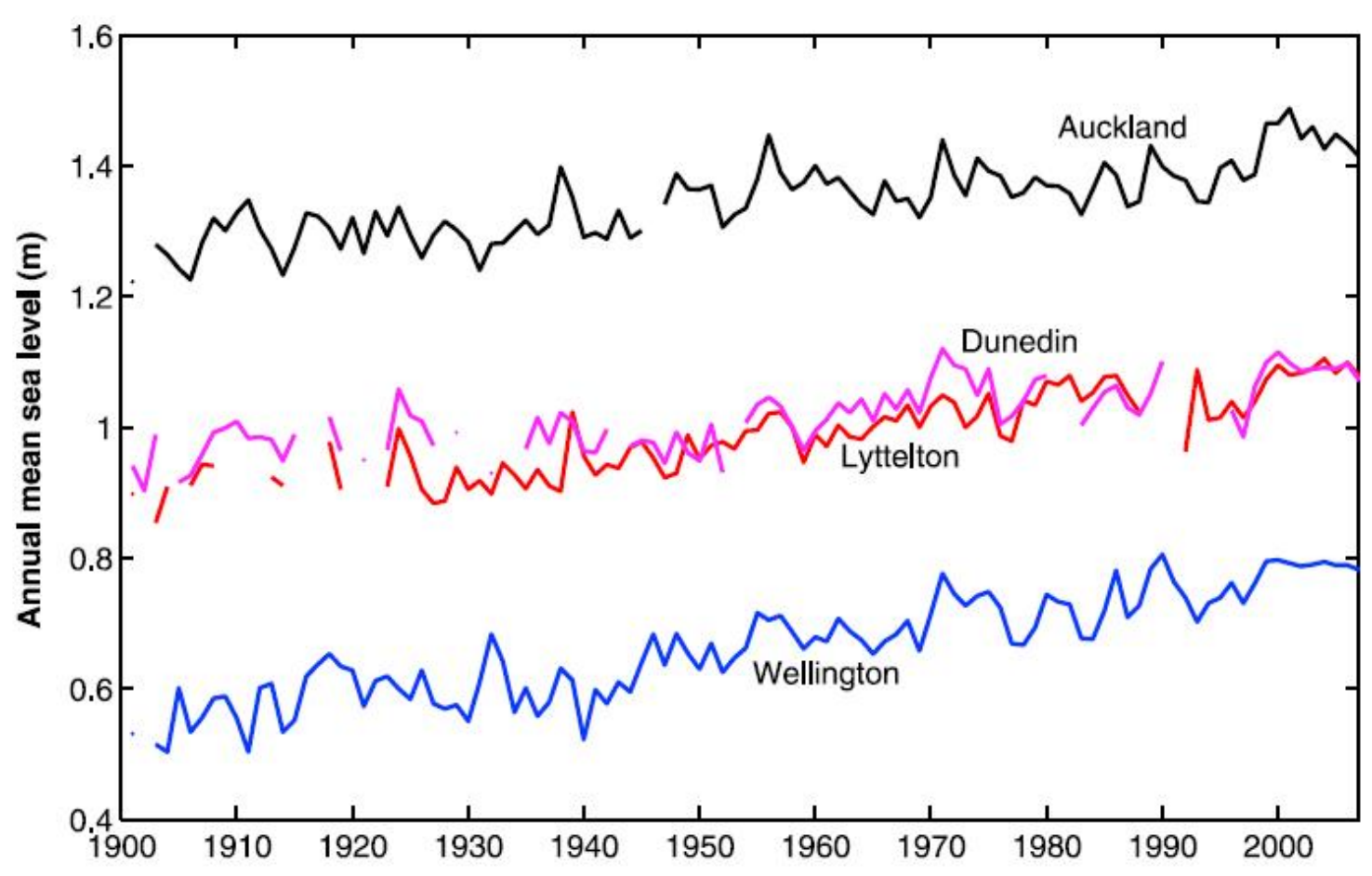

Figure 3.2: Annual mean sea level estimates from tide gauge data at Auckland, Wellington, Lyttelton and Dunedin. From Hannah and Bell (2012). 


\subsection{NZGeoid2005: local vertical datum unification}

To unify the local vertical datums and to establish a single vertical datum across New Zealand a quasigeoid was computed in 2005 (NZGeoid2005) from Earth Gravity Model 1996 (EGM96), the New Zealand terrestrial gravity network and shipborne gravity data (Amos, 2007). A simple offset for each of the LVD was estimated from this quasigeoid model to be used to translate normal-orthometric heights from one of the local vertical datums to another. To determine the offset for each of the LVD the following iterative approach was taken.

1. The terrestrial gravity measurements were all initially reduced using normal orthometric heights in terms of their local vertical datum, as seen in figure 3.1. A quasigeoid was then determined from the reduced terrestrial gravity values.

2. The estimated normal-orthometric height from the quasigeoid was then computed at the locations of precise leveling observations, the mean and standard deviation of the height difference between the quasigeoid normal-orthometric height and LVD normal-orthometric height was then estimated.

3. The mean height difference was then taken as the offset $\tilde{o}$ and was used to re-evaluate the gravity anomalies. The re-evaluated gravity anomaly, $\Delta \tilde{g}$ is given by,

$$
\Delta \tilde{g}=\Delta g+0.3086 \tilde{o}
$$

where $\Delta g$ is the initial estimated gravity anomaly in terms of the LVD and $\tilde{o}$ is given in metres.

These steps were then repeated until convergence of the offsets was reached which took one repetition. The quasigeoid NZGeoid2005 was recomputed in 2009 from more contemporary and accurate data and is used as the reference surface for New Zealand Vertical Datum 2009 (NZVD2009). 


\subsection{NZVD2009}

NZVD2009 is defined as a normal-orthometric height system, with the reference surface being the New Zealand quasigeoid 2009 (NZGeoid2009) using GRS80 as the reference ellipsoid/normal gravity field and is in terms of the zero-tide system.

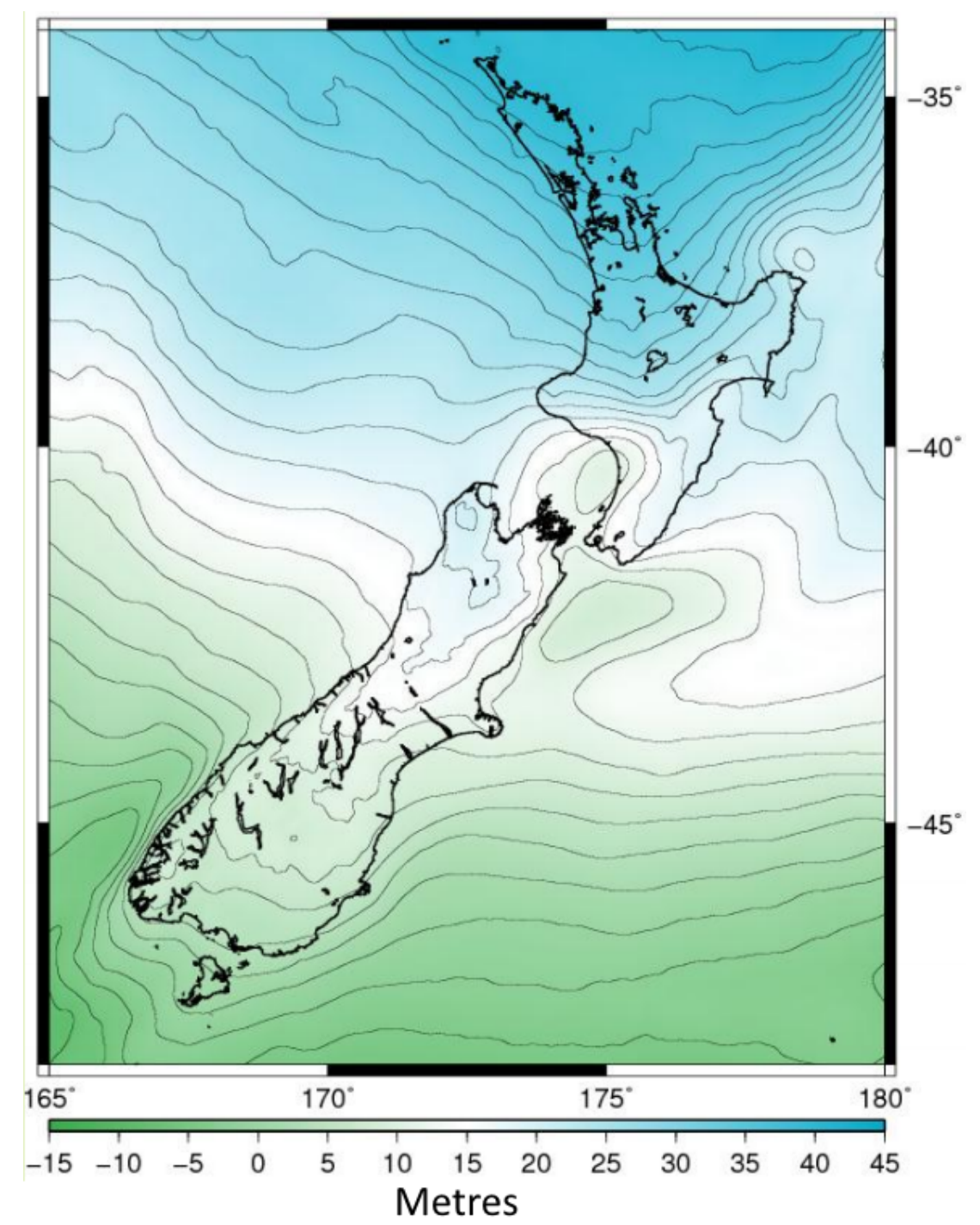

Figure 3.3: NZGeoid2009, the quasigeoid used as the reference surface for New Zealand Vertical Datum 2009.

It is the current, quasigeoid-based, height reference system (i.e. relative to the Earth's gravity field) that is used in New Zealand. This vertical datum was the first quasigeoidbased height system to be officially implemented as a national datum world-wide and the first consistent vertical datum to be used in New Zealand. It most notably offers an improvement on the previous convention of using local mean sea level tide-gauge based height systems. 
NZGeoid2009 ranges from 45 metres in the north $(35 \mathrm{~S})$ to -15 metres in the south $(50 \mathrm{~S})$ and is defined between $160^{\circ} \mathrm{E}$ to $190^{\circ} \mathrm{E}$ and from $25^{\circ} \mathrm{S}$ to $60^{\circ} \mathrm{S}$ at a spatial resolution of 1' $\times 1$ '. The technical report by Claessens et al. (2009), gives the following a list of the data sets which were used in the computation of NZGeoid2009.

1. Land gravity anomalies: Land gravity anomalies were computed from the 40,737 terrestrial gravity network data which covers the North, South, Stewart, Chatham and Pitt Islands. These data are described in detail in section 4.1.

2. Terrain correction data: 62 individual tiles $\left(1^{\circ} \times 1^{\circ}\right)$ with terrain corrections given at a 56 metre horizontal resolution. The terrain corrections were used to calculate the refined Bouguer anomalies necessary to grid the gravity data. The corrections were determined using prism integration described in Amos (2007).

3. DNSC08: A satellite altimetry based free air anomaly grid, produced by the Danish National Space Centre, used at a spatial resolution of $1^{\prime} \times 1$ '. It was used for two purposes. Firstly, it was merged with the terrestrial free air anomalies using a high resolution mask around the coast line so that any extrapolation when griding the terrestrial data could be avoided. Secondly, it was used to specify gravity anomalies over the ocean in place of the marine gravity observations, which were used to compute NZGeoid2005, due to its higher accuracy.

4. Digital elevation model (DEM): A high resolution digital elevation model gridded at a resolution of 56 metres. It was used to reconstruct the free air anomalies from the gridded refined Bouguer anomalies, as described by Featherstone and Kirby (2000).

5. EGM2008: The most contemporary and accurate global gravity model (at the time of computation). It is complete to degree and order 2160 , which corresponds to a wavelength of approximately $9 \mathrm{~km}$. It was used to provide the reference long and medium wavelength gravity and height anomalies in the remove-compute-restore stages in the processing. This global gravity model is described in detail in section 5.1 .

6. GPS/leveling data: These data were used to assess the accuracy of the determined quasigeoid and most importantly were used to determine the LVD offsets in the iterative step to determine local vertical datum offsets (as was done for NZGeoid2005). 
Processing steps taken to compute NZGeoid2009: The following flow diagram, adapted from Classens et al. (2009), demonstrates the processing steps taken in the computation of NZGeoid2009. The numbered cylinders correspond to the input data described in the previous section and the letters A-M describe the processing steps which were taken.

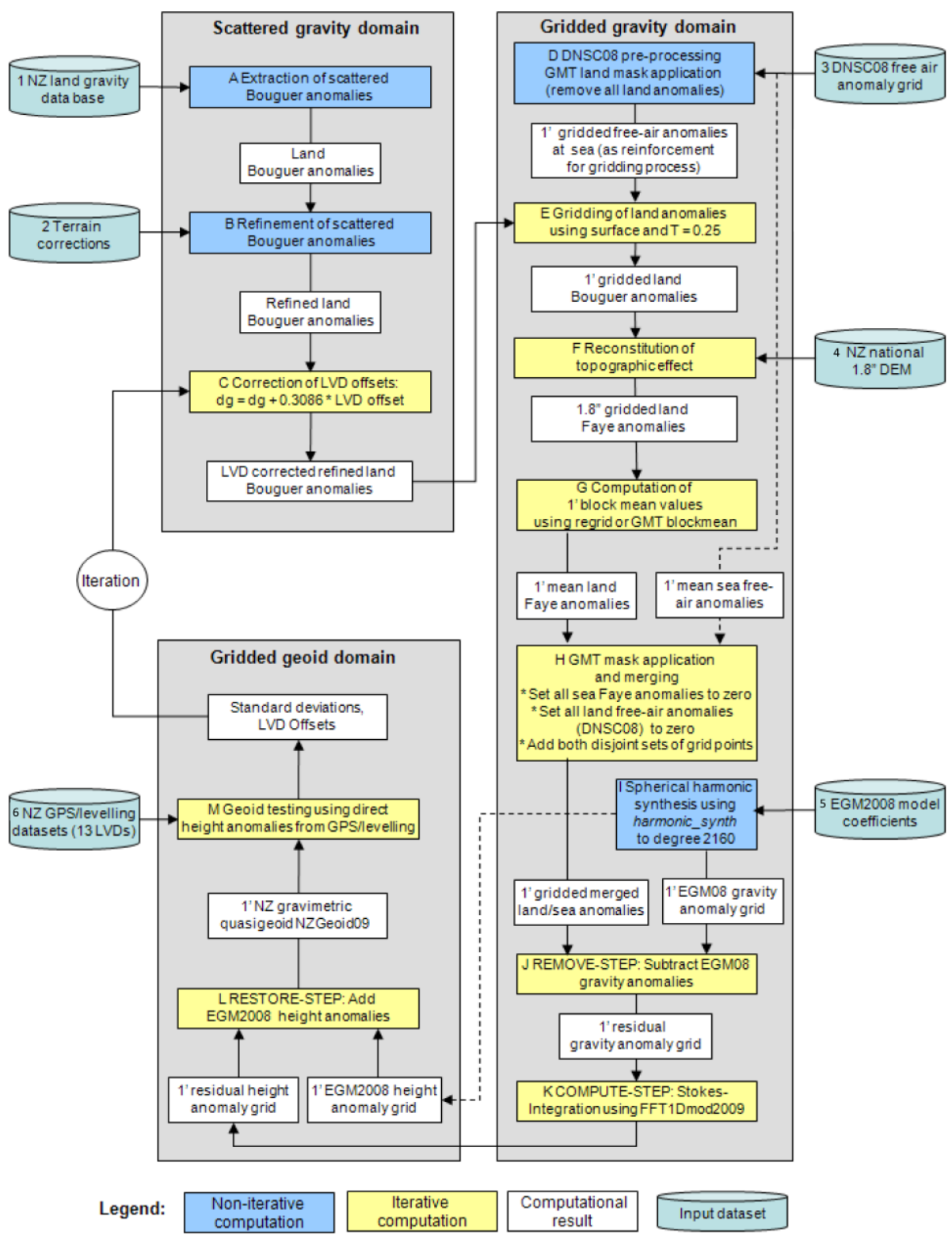

Figure 3.4: The processing steps taken in the computation of NZGeoid2009. - adapted from Classens et al. (2009) 
Following Classens et al. (2009), the following list describes the steps taken at each of the A-M steps in the flow chart above.

A. The land gravity measurements were reduced to scattered Bouguer anomalies, each in terms of their LVD normal-orthometric height.

B. The $56 \mathrm{~m}$ resolution terrain corrections from $1^{\circ} \times 1^{\circ}$ grids were then interpolated to the location of the scattered terrestrial data points. Then interpolated terrain corrections were added to the scattered Bouguer terrestrial gravity anomalies to obtain refined Bouguer anomalies.

C. The offset determined for the LVD was applied to the scattered refined Bouguer anomalies. Initially all offsets were set to zero.

D. Data from the $1^{\prime} \times 1^{\prime}$ grid of satellite altimetry derived free air anomalies from the DNSC08 gravity model were extracted inside the quasi computation area $\left(160^{\circ} \mathrm{E}\right.$ to $190^{\circ} \mathrm{E}$ and $25^{\circ} \mathrm{S}$ to $60^{\circ} \mathrm{S}$ ) and points inside the New Zealand coast line were removed using a high resolution mask.

E. The scattered refined Bouguer anomalies were temporarily augmented with the masked DNSC08 gravity model and then gridded on a $1^{\prime} \times 1$ ' grid. This was performed in GMT using the surface function, which employs continuous curvature splines with a user-define tension factor, chosen to be 0.25 . The temporary augmentation with the DNSC08 gravity model was performed to reduce the unwanted effects of extrapolation beyond the convex hull to the terrestrial data.

F. The high resolution digital elevation model was then used to reconstruct the effect of topography to obtain Faye anomalies. Gridding the refined Bouguer anomalies and then reconstructing the effect of topography using the DEM to obtain gridded Faye anomalies is more robust than just computing and then gridding the Faye anomalies directly from the terrestrial observations. This is because the high frequency content of the topography, which is not present in the refined Bouguer anomalies, can cause aliasing when griding Faye anomalies directly (Amos, 2007, Featherstone, 2000). This gives a grid with a resolution of $56 \mathrm{~m} \times 56 \mathrm{~m}$.

G. The high resolution grid of Faye anomalies was then interpolated back onto the coarser $1^{\prime} \times 1$ ' grid. This was performed by taking a mean of the data inside each cell. For data points on the high resolution grid which are partially inside one of the cells on the coarser grid, an appropriate weighting was assigned when computing the average.

H. The terrestrial Faye anomalies were then augmented with the DNSC08 gravity model using the GMT function gridlandmask to remove any data inside the coastline for the DNSC08 gravity anomaly model and outside the coastline for the terrestrial measurements. This gives a final $1^{\prime} \times 1$ ' grid of gravity anomalies inside the quasigeoid computation area $\left(160^{\circ} \mathrm{E}\right.$ to $190^{\circ} \mathrm{E}$ and $25^{\circ} \mathrm{S}$ to $\left.60^{\circ} \mathrm{S}\right)$.

I. A gravity anomaly grid $\left(1^{\prime} \times 1^{\prime}\right)$ from the EGM2008 coefficients (using coefficients of degree and order from 2 to 2160) was then computed over the quasigeoid computation area. The gravity values on the coarse $1^{\prime} \times 1$ ' grid were computed using area means 
with the areas (grid cell size) determined using an ellipsoidal approximation. All of the gravity anomalies were computed using the "zero-tide" system.

J. The grid of gravity anomalies generated from the EGM2008 coefficients was then algebraically subtracted from the grid of augmented DNSC08 and terrestrial anomalies. The residual signal had a mean of $0.1 \mathrm{mGal}$ and standard deviation of $4.69 \mathrm{mGal}$, with a range of about $330 \mathrm{mGal}$ for the large outliers (typically through areas of rough topography).

K. Using the gridded residual gravity anomaly signal the residual quasigeoid height anomalies were computed by Stokes' formula. Here the residual quasigeoid heights depended upon the maximum spherical harmonic degree removed from the Stokes' kernel, $L$, and the integration cap size $\psi_{0}$. The parameters were optimised by seeking a best fit solution to the GPS/leveling heights. $\left(L=40, \psi_{0}=2.5\right)$,

L. Height anomalies from the EGM2008 model were then added back to the derived residual height anomalies determined in the previous step to obtain the full quasigeoid height.

M. The quasigeoid height anomalies determined in step $\mathbf{L}$ were then compared to 1422 directly observed height anomalies from GPS/leveling observations. The mean and standard deviation of the differences were then determined for each of the LVD. The means were then taken as the offsets and then steps $\mathbf{C}$ to $\mathbf{M}$ were repeated until the offsets converged.

Convergence was reached in one iteration which indicates that it was not necessary to repeat the calculations.

Accuracy assessment: In this subsection an accuracy assessment of NZGeoid2009 has been performed by looking at the difference between NZGeoid2009 height anomalies to those determined from the New Zealand leveling/GPS network. The leveling normalorthometric measurements give successive relative height differences which are referenced to one of thirteen mean sea level estimates determined from tide gauge measurements. The mean sea level estimate determined from the tide gauge measurements will differ from NZGeoid2009 by some amount, this will propagate through the leveling measurements that are referenced to it as a constant offset. For this reason a mean is subtracted from the leveling/GPS height anomalies and NZGeoid2009 differences separately for data in each LVD (Amos, 2007).

The leveling/GPS height anomalies have been compared to NZGeoid2009 before and after the LVD offsets were added and the agreement between the data sets has been assessed visually and statistically in both cases. With the addition of the local vertical datum offsets, the agreement of the leveling/GPS derived height anomalies reduced the mean to $-2 \mathrm{~cm}$ from $-32 \mathrm{~cm}$ and reduced the standard deviation (estimated from the inner $68 \%$ of the distribution to avoid the effect of outliers, following appendix B) to $4.8 \mathrm{~cm}$ from $12.8 \mathrm{~cm}$. The improvement is also evident visually with the removal of systematic 
trends in the difference between the leveling/GPS heights and NZGeoid2009.

For each leveling observation, $H^{N-O}$ (normal orthometric height), in the primary precise leveling network there is an accompanying GPS ellipsoidal height $(h)$ observation. The height anomaly, (denoted from here on) $N$, which is the separation of the quasigeoid from the ellipsoid, is calculated at for each pair of GPS and leveling observations by,

$$
N=h-H^{N-O}
$$

The following figure shows the height anomalies determined from the primary precise leveling network and GPS observations without the adjustment for the LVD offsets. As for NZGeoid2009 there is a negative north south trend and a range of 40 metres to 5 metres.

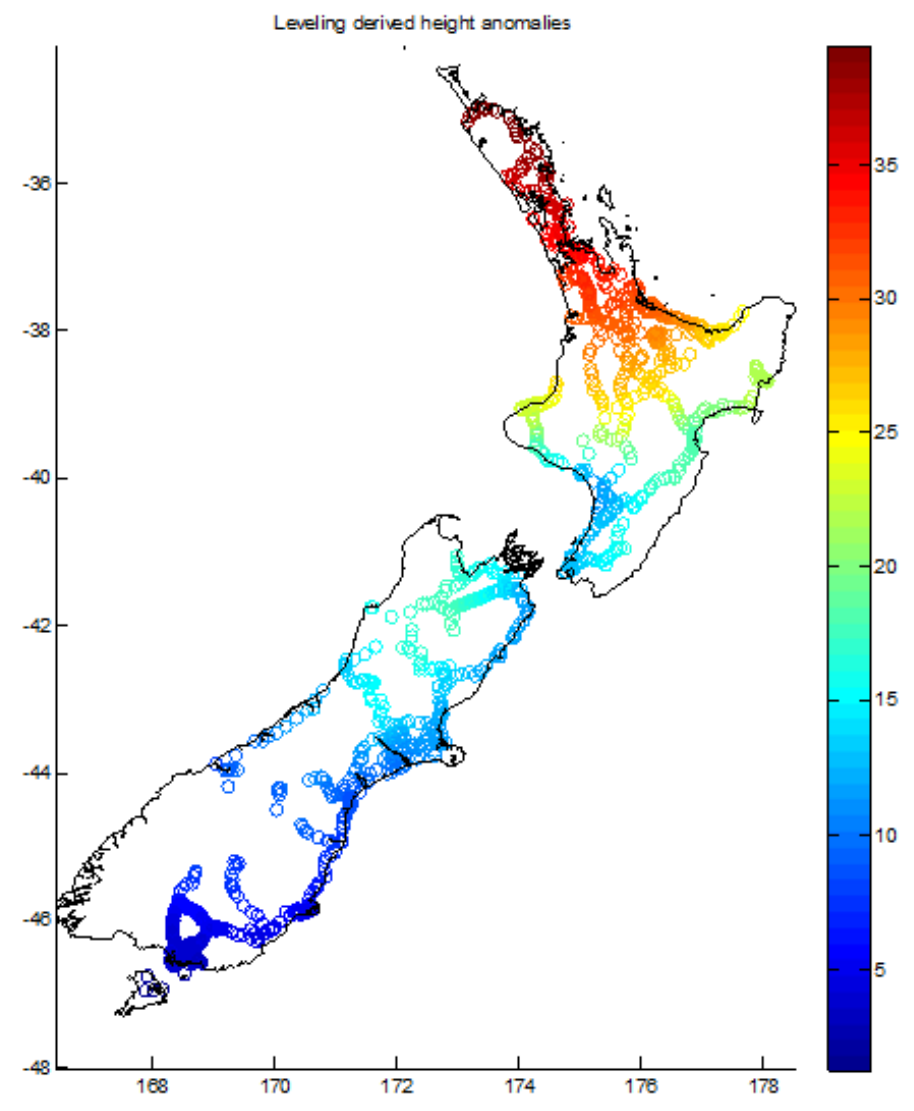

Figure 3.5: Height anomalies derived from the primary precise leveling network and GPS observations. The colour scale is in metres.

The following two figures show the difference between the leveling derived height anomalies and NZGeoid2009 before and after the published offsets are applied. 

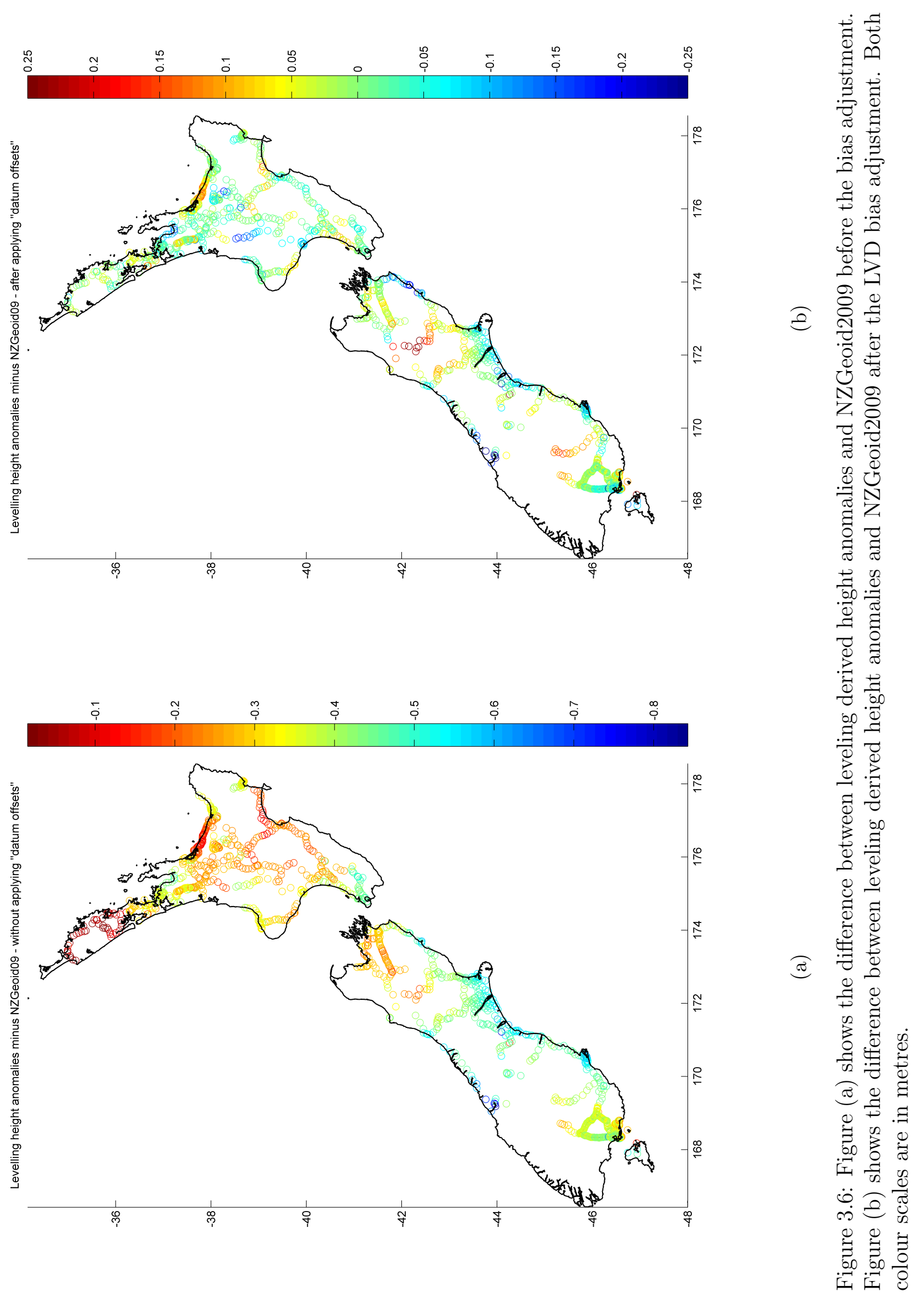
After the bias adjustment for the LVD leveling data, the agreement between the leveling derived height anomalies and NZGeoid2009 improves. This can be seen in the previous figures with differences between the leveling data and NZGeoid2009 no longer correlated for separate LVD's.

The following empirical cumulative distribution function (cdf) plot (Zwillinger, 2010) demonstrates the improvement in the distribution of the difference between the leveling derived height anomalies and NZGeoid2009 before and after the LVD bias adjustment. Before the bias adjustment (blue) the agreement between the leveling derived height anomalies and NZGeoid2009 has a mean of $-34.65 \mathrm{~cm}$ and a standard deviation of 12.8 $\mathrm{cm}$. After the bias adjustment (red) the agreement between the leveling derived height anomalies and NZGeoid2009 has a mean of $-2 \mathrm{~cm}$ and a standard deviation of $4.8 \mathrm{~cm}$. The standard deviations were estimated from the inner $68 \%$ of the distributions since they are leptokurtotic (long tailed), following appendix B.

The improvement in the agreement between the two data sets (leveling/GPS derived height anomalies and NZGeoid2009) indicates that the LVD bias adjustment is a necessary step and one which must be performed for any regional geoid to assess its accuracy.

\subsection{Summary}

In this chapter the concept of a vertical datum is defined. Descriptions of the leveling/GPS data available for the 13 local vertical datums, NZGeoid2005 and NZVD2009 is given.

Prior to the national geoid computation NZGeoid2005 New Zealand had 13 separate local vertical datums. The intention of the computed Geoid was to unify the local vertical datums by computing offsets for each of them from the consistent reference surface.

NZGeoid2009 superseded NZGeoid2005 and was based on the most accurate data available at the time. NZVD2009 is the vertical datum based on NZGeoid2009 and was the first of its kind world wide.

In this study's new assessment, NZGeoid2009 was shown to have an accuracy of 4.8 $\mathrm{cm}$ (standard deviation) on comparison to leveling data after local vertical datum offsets are applied. Applying the local vertical datum offsets was shown to be a crucial step in determining the accuracy of the geoid and should be applied for any newly created geoid. 


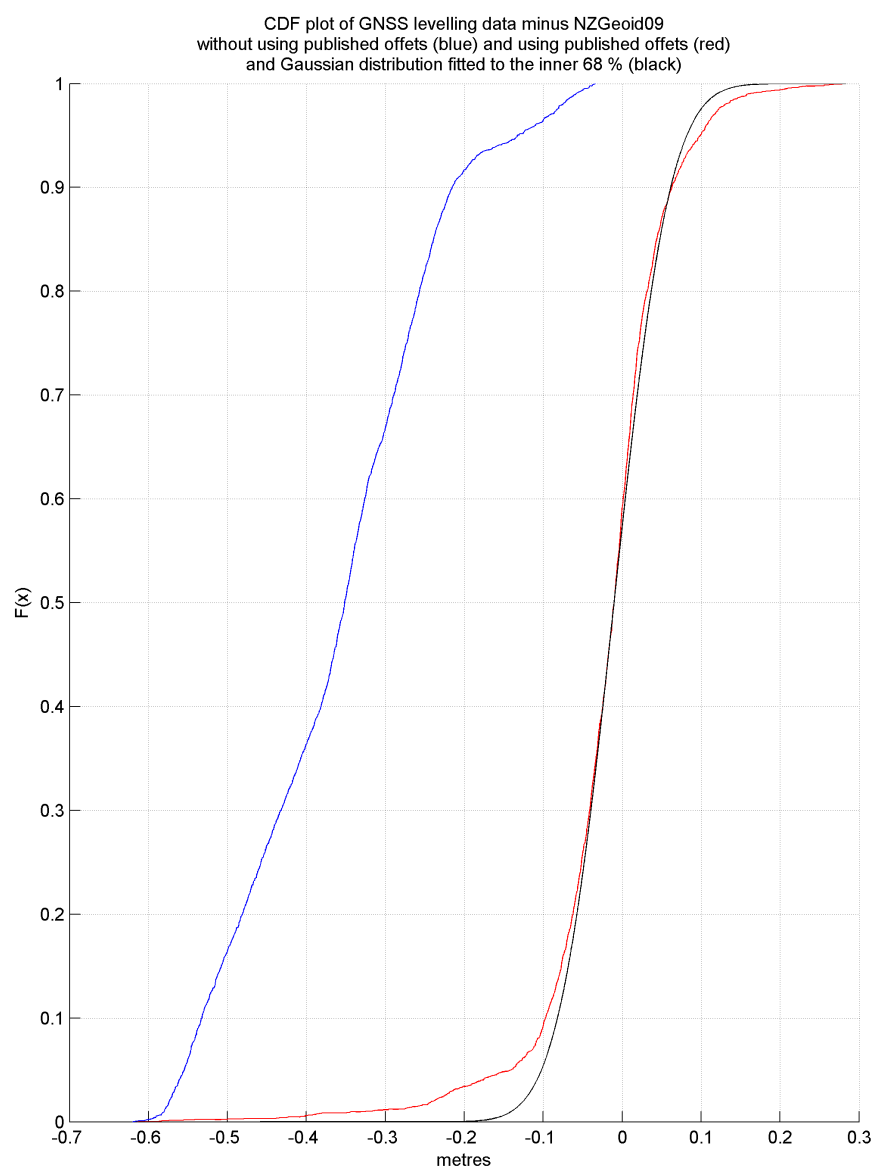

Figure 3.7: CDF plot of the difference between leveling derived height anomalies and NZGeoid2009 height anomalies before (blue) and after LVD bias adjustment (red), best fitting Gaussian cdf is in black. The LVD bias adjustment improves the standard deviation of the inner $68 \%$ of the distribution by around $8 \mathrm{~cm}$ and also reduces the overall bias of the distribution by $32 \mathrm{~cm}$ in absolute value. 


\section{Chapter 4}

\section{Terrestrial Gravity in New Zealand}

\subsection{Introduction}

This chapter gives an overview of the terrestrial gravity measurements in the GNS Science database. A description of the instrumentation used to collect the data is given and the varying spatial density of the measurements is discussed and displayed. Here, the gravity data are reprocessed and details of the formulas used to calculate refined Bouguer gravity anomalies are given. This is important to ensure consistency in the data reductions prior to computing the new national geoid (chapter 8). Finally, an accuracy assessment of the data has been performed.

\subsection{GNS Science terrestrial gravity database}

GNS Science maintains a database of terrestrial gravity measurements made across New Zealand. The gravity network was established from the 1940's. The network consists of gravity measurements at approximately 40,000 locations nationwide which have been made by several different institutions with various gravimeters. However, it mostly consists of relative gravity readings made using Lacoste and Romberg D and G meters which are tied to the New Zealand primary gravity network by the least squares procedure described by Woodward and Carman (1984). In general the derived absolute gravity values from repeat relative gravity observations have an estimated accuracy of around $0.1 \mathrm{mGal}$.

The New Zealand primary network is a set of bench marks maintained by Land Information New Zealand. It was established from the 1960s to be a series points with a "known" absolute gravity value (Stagpoole, 2012). The benchmarks were reoccupied during the 1980s to account for any changes in their locations relative to roads and railway stations nearby and to replace benchmarks which had been destroyed.

The terrestrial gravity observations were used to compute NZGeoid2009 (chapter 3). However, they were not originally intended for geoid modeling and are not ideally suited to it since the database contains historic measurements and are sparse in rough topography. The spacing of the observations is on average about $7.5 \mathrm{~km}$ (Amos, 2007) and can be seen in figures 4.1 and 4.2 . 


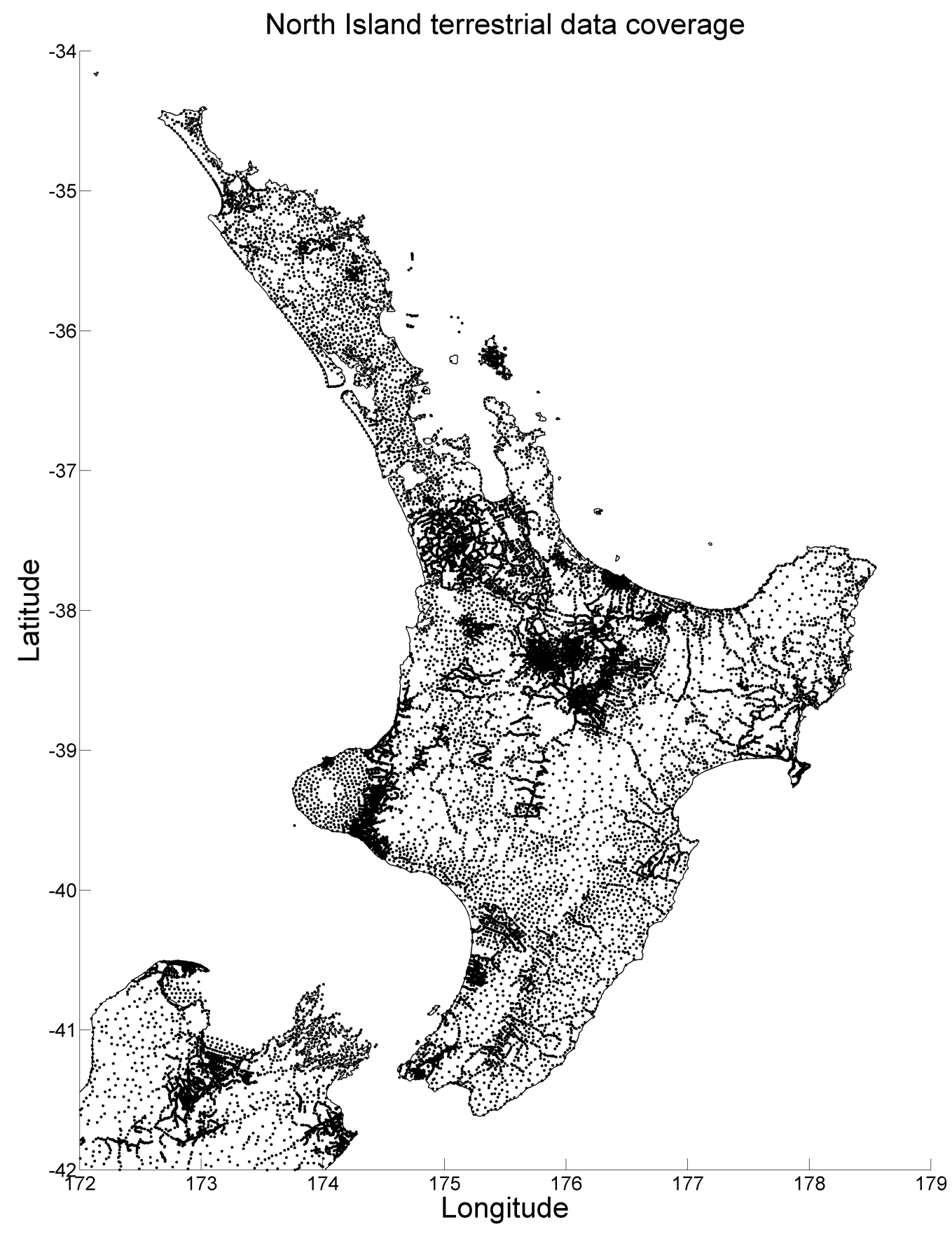

Figure 4.1: Terrestrial gravity data coverage over the North Island of New Zealand. The density of measurements can be seen to vary accross the island. In areas of scientific interest, such as the Taupo Volcanic Zone, the density increases whereas in rougher terrain, such as in the Opotiki region (in the East) the density reduces. 


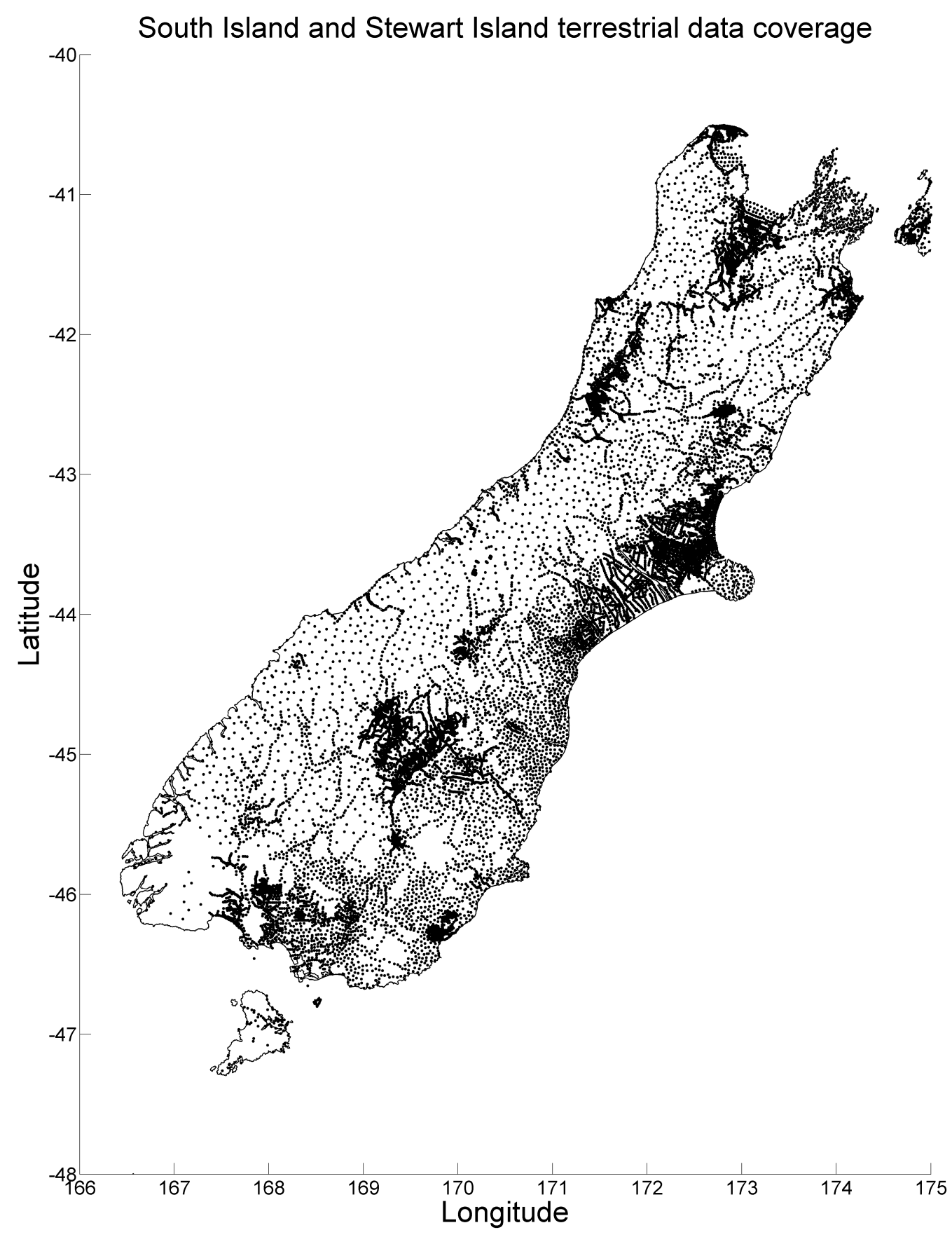

Figure 4.2: Terrestrial gravity data coverage over the South Island and Stewart Island of New Zealand. Where the topography is rough the data density reduces, for example through the ranges down the centre of the country or in Fiordland (in the South-West) where the data density drops to almost one measurement every $30 \mathrm{~km}$. In flatter regions, the data density is much higher, for example in Canterbury (centre-East). 


\subsection{Gravity reduction}

The database contains the absolute gravity, $g$, and position (latitude and longitude, and the normal orthometric height in terms of the local vertical datum) at each of the network locations, the gravitational effects of the normal gravity, $\gamma$, free air correction $\delta g_{F A}$, and the Bouguer slab and terrain corrections, $\delta g_{B S}$ and $\delta g_{T C}$. However over the years various conversion formulas, which are potentially inaccurate, have been used to transform historic normal gravity calculations to the modern standard at the time (Stagpoole, 2012). In the calculations that follow, this study recomputes a new set of corrected terrestrial gravity data to ensure consistency in the application of correction terms.

The normal gravity corrections have all been made with respect to the GRS80 reference ellipsoid which has the following necessary parameters;

- $a=6378137 m$ : The equatorial radius

- $b=6356752.3141 m$ : The polar radius

- $\gamma_{a}=9.7803267715 \mathrm{~m} \mathrm{~s}^{-2}$ : The absolute gravity at the equator

- $k=0.001931851$ 353: The normal gravity constant

- $e^{2}=1-b^{2} / a^{2}=0.00669438002290$ : The first eccentricity squared.

The recomputed corrections are;

1. Normal gravity $\gamma(\phi)$ : calculated by the Moritz (1980) formula, $\gamma(\phi)=\gamma_{a} \frac{1+k \sin ^{2}(\phi)}{\sqrt{1-e^{2} \sin ^{2}(\phi)}} \times$ $10^{5} \mathrm{mGal}$ (section 2.2.1).

2. The free air correction $\left(\delta g_{F A}\right): \delta g_{F A}=-\frac{2 \gamma}{a}\left(1+f+m(-3 f+5 / 2 m) \sin ^{2}(\phi)\right) H+$ $\frac{3 \gamma_{a} H^{2}}{a^{2}}(\mathrm{mGal})$, where $H$ is the normal-orthometric height given in metres from the database.

3. The infinite Bouguer slab correction $\left(\delta g_{B S}\right): 0.0419 \rho H$ (mGal) where $\rho$ is the standard density $\left(2.67 \mathrm{Mg} / \mathrm{m}^{3}\right)$ and again $H$ is the normal-orthometric height given in metres from the database.

4. The terrain correction $\left(\delta g_{T C}\right)$ : Calculated using field observations and the Hammer prism method with an $8 \mathrm{~m}$ (out to $2160 \mathrm{~m}$ ) and a $64 \mathrm{~m}$ DEM (from $2160 \mathrm{~m}$ to 120 $\mathrm{km})$. This is described in detail in the following section .

The corrections can be used to transform the absolute gravity values $g$ in the GNS Science gravity database to refined Bouguer anomalies $\Delta g_{B}$ by the following equation,

$$
\Delta g_{B}=g-\gamma+\delta g_{F A}-\delta g_{B S}+\delta g_{T C} .
$$

Since the absolute gravity observations were in the ISGN71 standard (which is meantide), the following transformation (as given in chapter 3 ) has been applied to obtain the data in the zero-tide system,

$$
\Delta g_{z}=\Delta g_{m}+3.04-9.12 \sin ^{2}(\phi) .
$$


Figure 4.3 shows the free air gravity anomalies reduced using formulas from points 1 ) and 2) (i.e. prior to the terrain Bouguer Slab and terrain corrections) across the North Island, South Island and Stewart Island on a 1 arc minute grid, gridded using a linear interpolation algorithm.

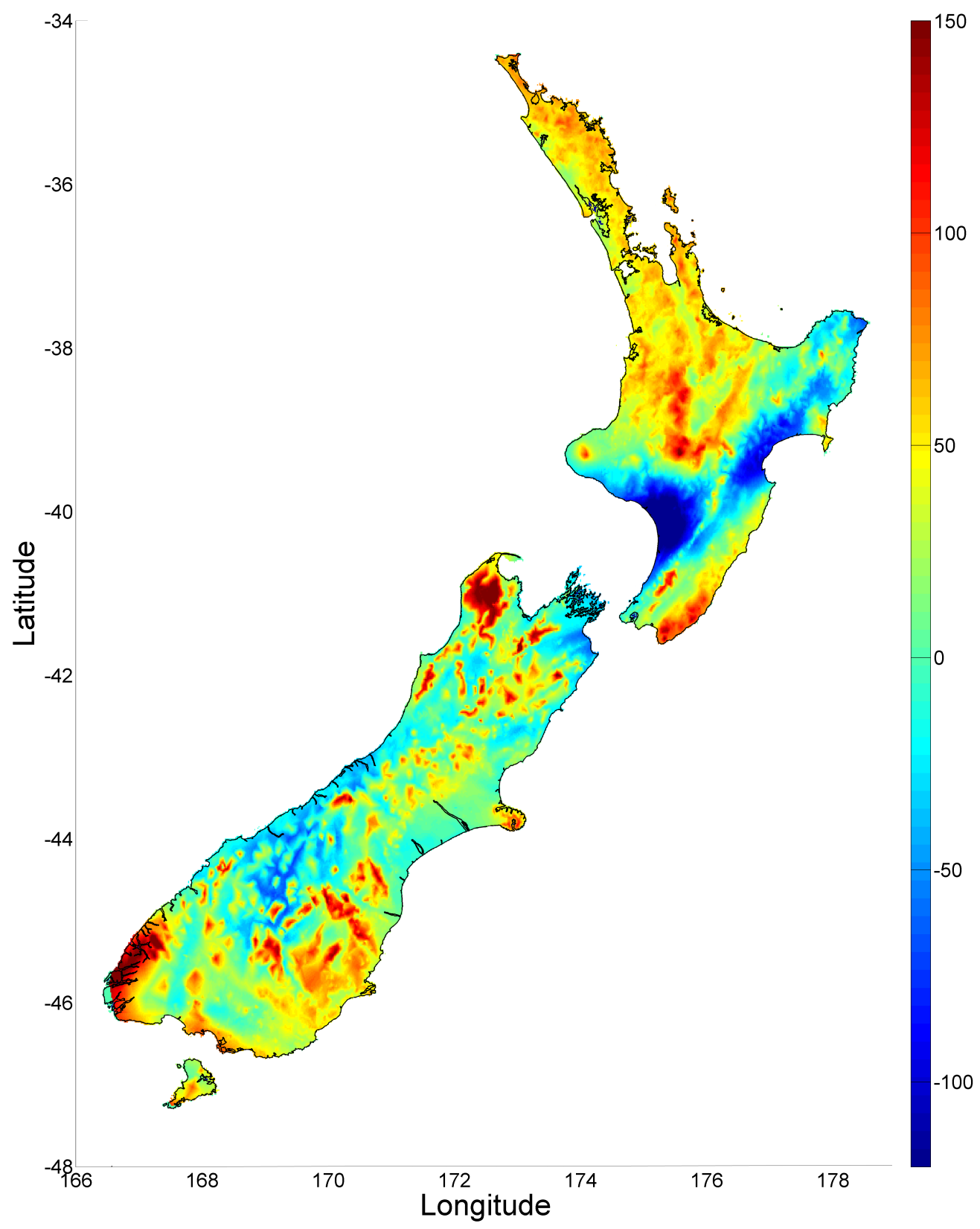

Figure 4.3: Recomputed terrestrial free air anomaly data over the North, South and Stewart Island, gridded at 1 arc minute for illustrative purposes using a linear interpolation algorithm. The scale is in $\mathrm{mGal}\left(10^{-5} \mathrm{~m} / \mathrm{s}^{2}\right)$. 
In addition to the data coverage on the North, South and Stewart Island, 285 gravity observations on Chatham Island and Pitt Island (to the east of mainland New Zealand) are also available in the GNS Science database. The data have also been reduced using the same formulas as above and the free air anomaly (i.e. prior to the Bouguer Slab and terrain corrections) can be seen in figure 4.4.

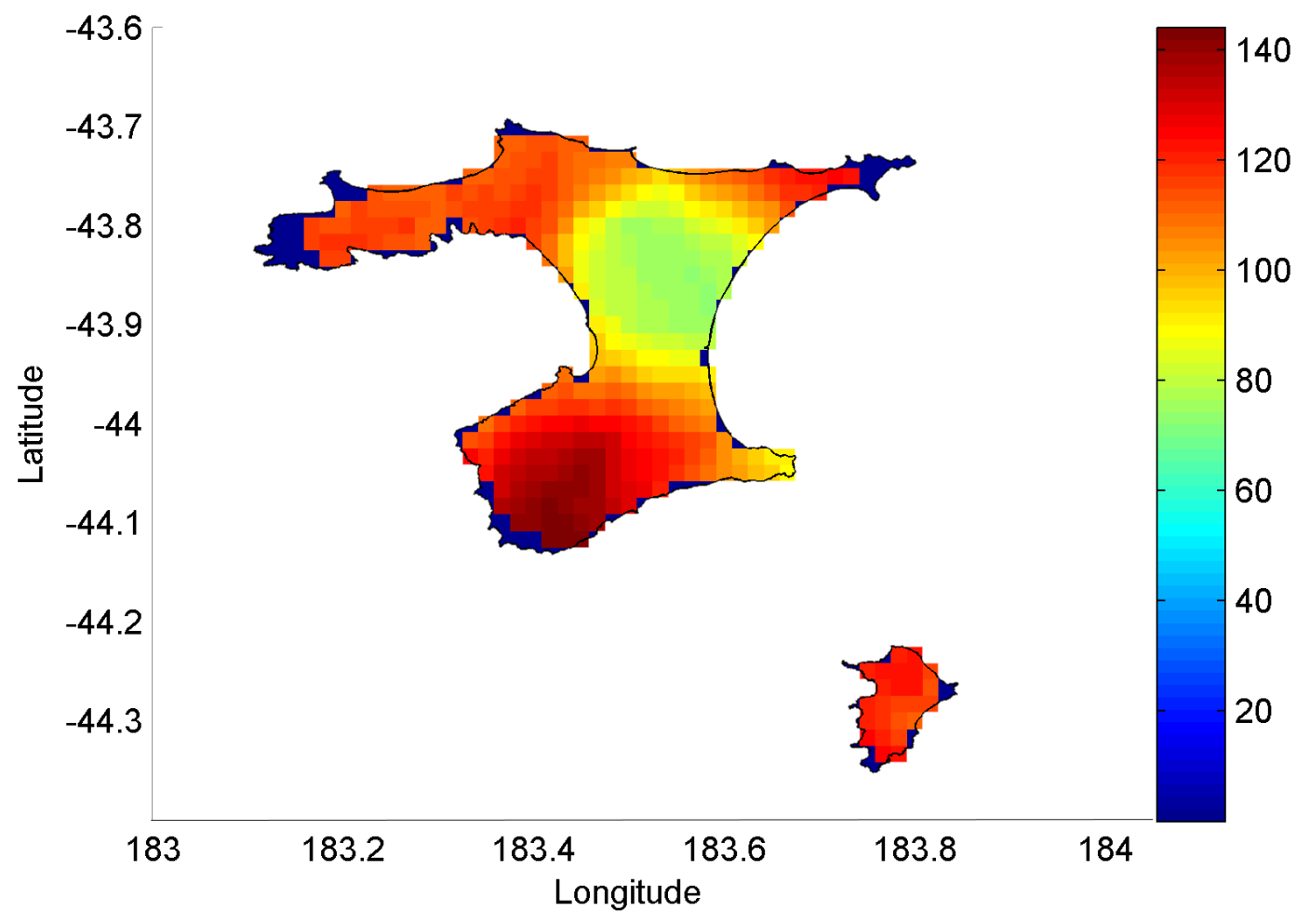

Figure 4.4: Terrestrial free air anomaly data over Chatham (top) and Pitt (bottom) Islands, gridded at 1 arc minute for illustrative purposes using a linear interpolation algorithm. The scale is in $\mathrm{mGal}\left(10^{-5} \mathrm{~m} / \mathrm{s}^{2}\right)$.

\subsection{Terrain Corrections}

The GNS Science terrestrial gravity database contains inner zone terrain corrections computed from field observations using the Hammer zone method (Hammer, 1939, see chapter 3 ) and outer zone terrain corrections (from the edge of the inner zone out to $-21900 \mathrm{~m}$ ). The outer zone corrections were computed with the Nagy prism method and a coarse digital elevation model.

Horizontal positions of the gravity observations in the database have frequently been read from 1:250000 map sheets; these readings are only accurate to around $100 \mathrm{~m}$. Inaccurate horizontal positioning causes large errors when computing inner terrain corrections from a DEM, particularly in steep terrain. 
To attempt to reduce errors propagating from mis-locating the gravity observations when computing the terrain corrections, a composite set of terrain corrections calculated from the DEM and field observations has been derived. Three terrain corrections, $\delta g_{1}, \delta g_{2}$ and $\delta g_{3}$, have first been calculated. $\delta g_{1}$ was computed from the $8 \mathrm{~m}$ digital elevation model from the site location out to Hammer zone $\mathrm{H}(2610 \mathrm{~m})$ and $\delta g_{2}$ was computed from the $64 \mathrm{~m}$ digital elevation model from $2610 \mathrm{~m}$ out to $120 \mathrm{~km}$.

$\delta g_{3}$ was computed from the $8 \mathrm{~m}$ digital elevation model from the observation location out to the far edge (zone D, i.e. $170 \mathrm{~m}$, for almost all of the marks) of the recorded inner zone terrain correction, $\delta g_{G N S}$, in the GNS Science data base. Figure 4.5 shows a plot of the terrain corrections given in the GNS Science database against the DEM derived inner terrain corrections.

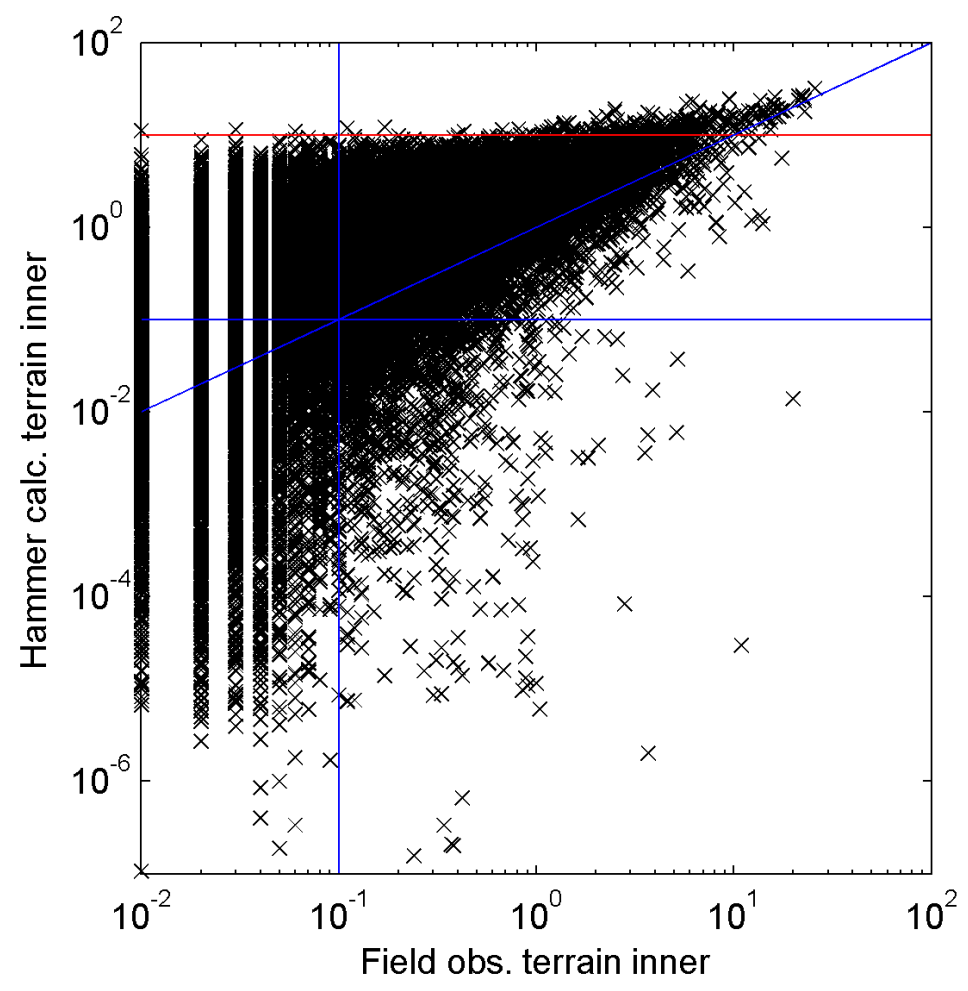

Figure 4.5: Field observation terrain corrections plotted against the inner zone terrain corrections computed by the Hammer prism method. Reference lines are shown at 0.1 $\mathrm{mGal}$ and Hammer $=$ Field obs. in blue and $10 \mathrm{mGal}$ in red. The scale is in $\mathrm{mGal}\left(10^{-5}\right.$ $\left.\mathrm{m} / \mathrm{s}^{2}\right)$.

The difference between the observed terrain corrections and those calculated from the DEM can be explained as follows.

- Where the field observation derived terrain correction is small (e.g. less than 0.1 mGal) then it is likely to be correct, since it would be easy to estimate the topogra- 
phy by eye where it is generally flat - in this circumstance it would be advantageous to use the field observations for the inner zone terrain correction.

- Where the field observations derived terrain correction is large (e.g. greater than $0.1 \mathrm{mGal}$ ) but the DEM derived terrain correction is small (e.g. less than 0.1 mGal), then the discrepancy is likely due to an over estimation of the topography in the field, in which case it would be better to use the DEM derived inner terrain correction.

- Where both the field observations and DEM derived terrain corrections are large the topography will be strongly varying. In this circumstance small errors in the observation's recorded position will result in large changes in the inner terrain corrections. Similarly the DEM terrain correction is band limited in the near zone as mentioned in section 2.2.3. For these reasons it will be better to use those derived from field observations.

To account for these points a temporary set of measurements $\delta \hat{g}_{1}$ is calculated where,

$$
\delta \hat{g}_{1}=\delta g_{1}-\delta g_{3}
$$

then the revised terrain correction out to hammer zone $\mathrm{H}, \delta g_{H}$, is calculated by the following,

$$
\begin{gathered}
\delta g_{H}=\delta \hat{g}_{1}+\delta g_{G N S}, \text { if } \delta g_{G N S}<0.1 \\
\delta g_{H}=\delta \hat{g}_{1}+\delta g_{3}, \text { if } \delta g_{G N S} \geq 0.1 \text { and } \delta g_{3}<0.1 \\
\delta g_{H}=\delta \hat{g}_{1}+\delta g_{G N S}, \text { if } \delta g_{G N S} \geq 0.1 \text { and } \delta g_{3} \geq 0.1
\end{gathered}
$$

The optimal (following the points above) composite terrain correction, $\delta g_{T C}$ out to 120 $\mathrm{km}$ is then given by

$$
\delta g_{T C}=\delta g_{H}+\delta g_{2}
$$

The Bouguer anomaly value, $\Delta g_{B}$ has then been obtained for each of the terrestrial free air anomaly gravity values $\Delta g_{F A}=\Delta g_{F A}(x, y, h)$ by subtracting the vertical component of the gravitational attraction from a Bouguer slab, $\delta g_{B S}$ of thickness $h$ (using the recorded normal-orthometric height from the database) with infinite radius and adding the calculated the terrain correction. i.e.

$$
\Delta g_{B}=\Delta g_{F A}-\delta g_{B S}+\delta g_{T C}
$$


Figure 4.6 shows the refined Bouguer anomaly gridded at a 1 arc minute resolution using a linear interpolation algorithm over the North, South and Stewart Islands.

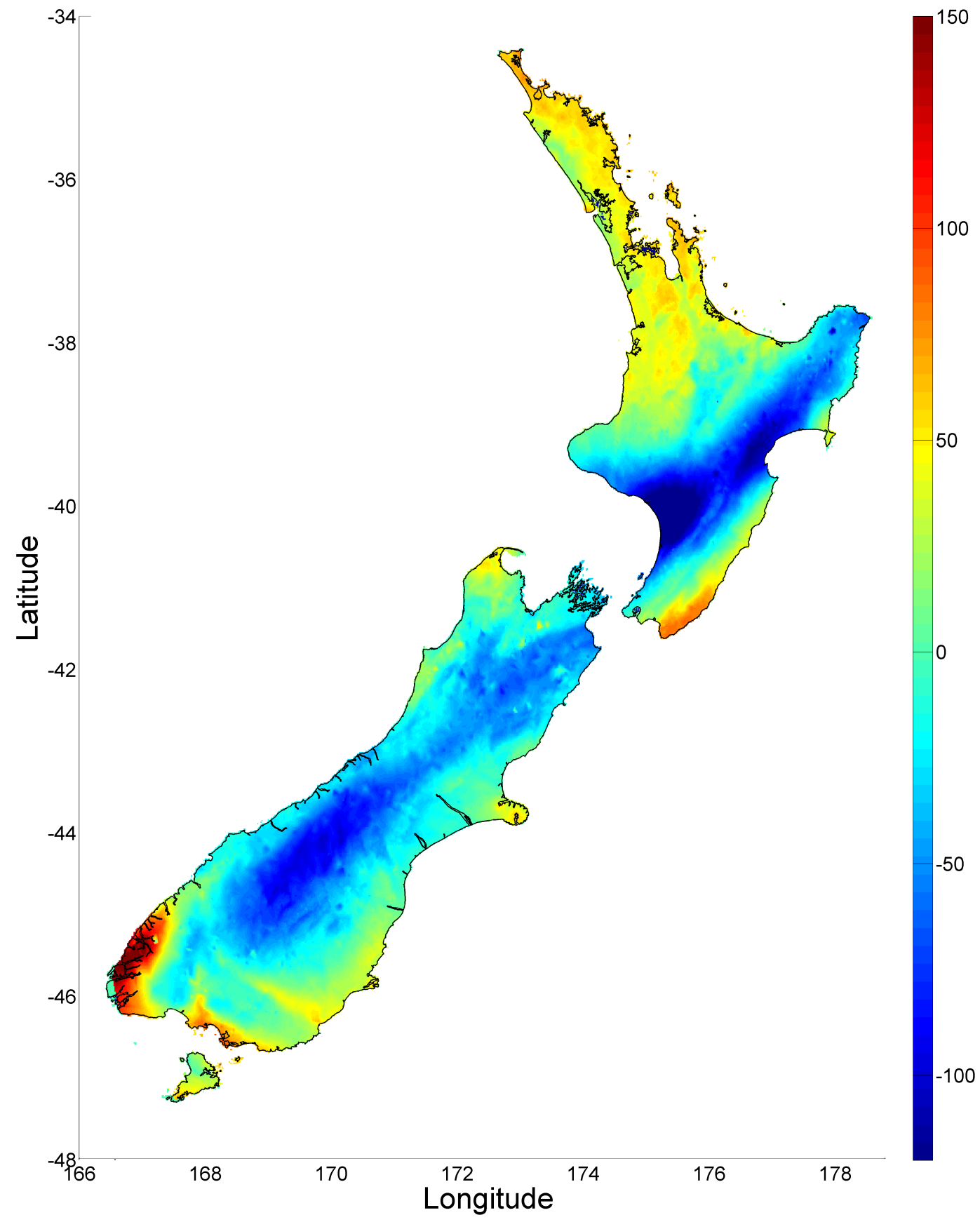

Figure 4.6: Terrestrial refined Bouguer anomaly data over the North, South and Stewart Island, gridded at 1 arc minute for illustrative purposes using a linear interpolation algorithm. The scale is in $\mathrm{mGal}\left(10^{-5} \mathrm{~m} / \mathrm{s}^{2}\right)$. 
For Chatham and Pitt Islands, the $8 \mathrm{~m}$ DEM was not available. For this reason the terrain corrections in the GNS Science database have been used explicitly. Figure 4.7 shows the refined Bouguer anomaly for the two islands.

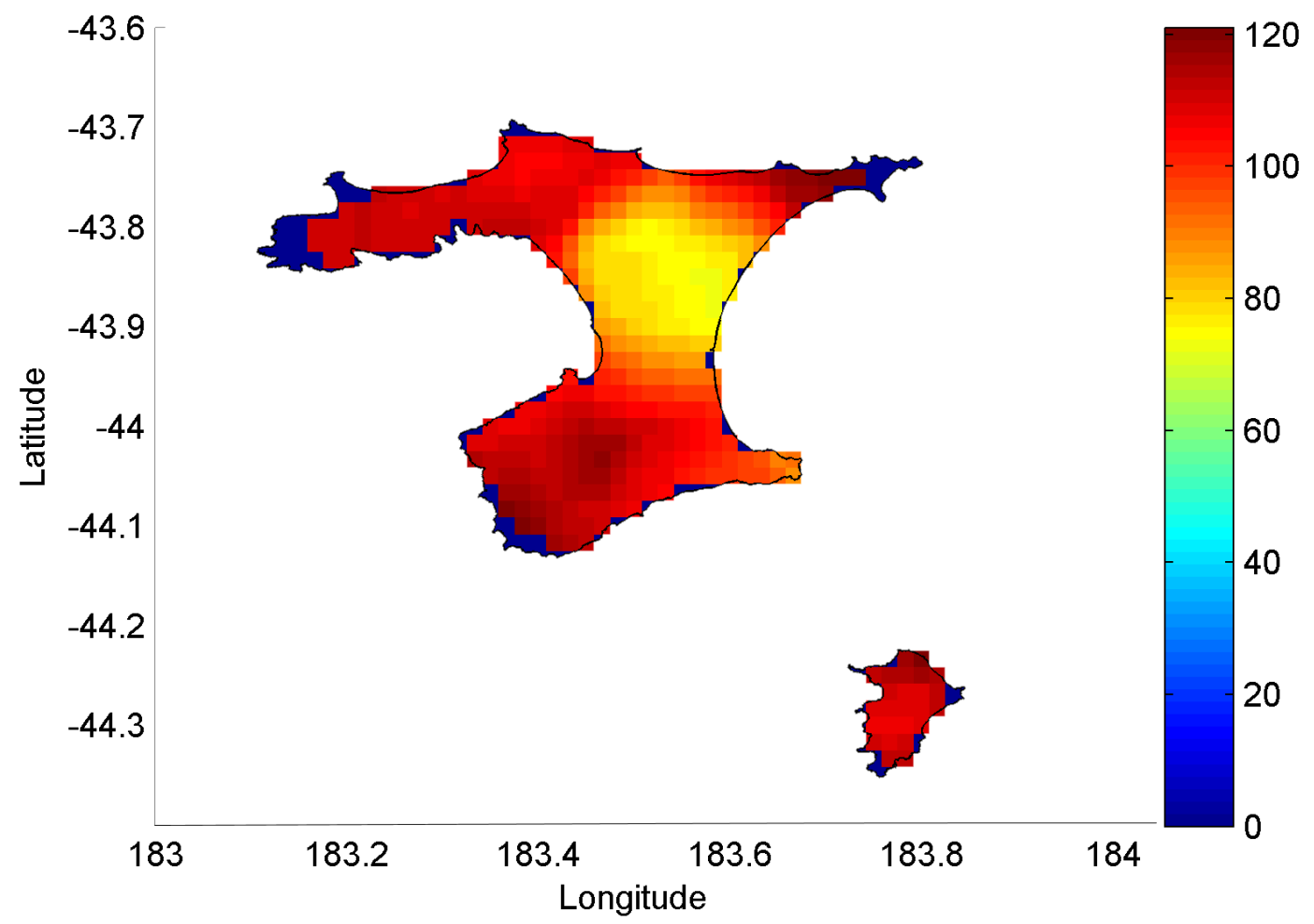

Figure 4.7: Terrestrial refined Bouguer anomaly data over Chatham (top) and Pitt (bottom) Islands, gridded at 1 arc minute for illustrative purposes using a linear interpolation algorithm. The scale is in $\mathrm{mGal}\left(10^{-5} \mathrm{~m} / \mathrm{s}^{2}\right)$.

\subsection{Accuracy assessment}

When calculating gravity anomalies from the absolute gravity values (at surveyed locations) the predominant cause of error is due to the recorded position of the observation sites (Stagpoole, 2012), and in particular the recorded vertical positions (Amos, 2007). In the GNS Science database the observation heights are given as normal-orthometric heights in metres but the heights are not derived by consistent means. Three height codes are used to specify the source of the recorded height and are as follows,

- 0 - Benchmark heights obtained by leveling and differential GPS (DGPS).

- 1 - Spot heights obtained from map sheets or by GPS.

- 2 - Barometric heights. 
Digital elevation model (DEM) heights can be useful for estimating the reliability of the prescribed heights in the database (Stagpoole, 2012). The $8 \mathrm{~m}$ DEM has been used to estimate the heights for each surveyed location in the GNS Science database. The difference between recorded heights and the DEM heights for locations with a height code of 0 (i.e. benchmarks) has a standard deviation ( fitted to the inner $68 \%$ of the distribution to reduce the effect of the long tails, see appendix B) of $7.5754 \mathrm{~m}$. This is shown in figure 4.8 of the empirical CDF plot of the differences (in blue) and the fitted distribution (in red).

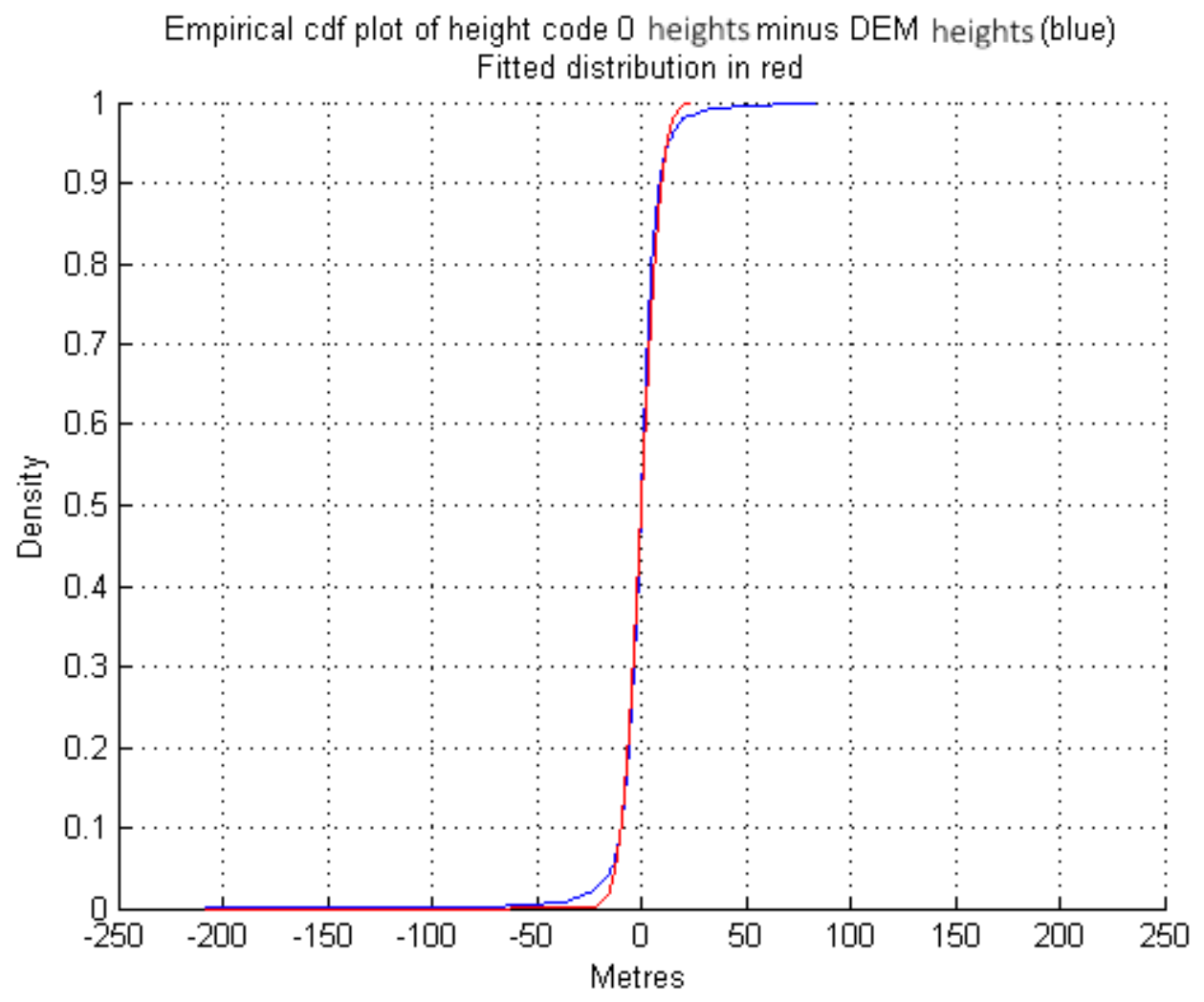

Figure 4.8: Empirical CDF of the heights with code 0 minus heights derived from the 8 $\mathrm{m}$ DEM (in blue) and distribution fitted to the inner $68 \%$ (in red).

The distribution shown above is the sum of two random distributions, the first is due to the distribution of errors in the DEM heights and the second is the error in the recorded heights in the GNS Science database. The recorded leveling/DGPS heights have a fixed estimated error with a standard deviation $\sigma_{0} \approx 10-15 \mathrm{~cm}$. This means the standard deviation of the DEM can be approximated as $\sigma_{D E M}=\sqrt{7.57^{2}-0.15^{2}} \approx 7.57$ to two decimal places.

The $8 \mathrm{~m}$ DEM heights have then been used to estimate heights for the gravity observations with height code 1 . The difference between recorded heights and the DEM heights for locations with a height code of 1 (i.e. spot heights) has a standard deviation ( fitted to the inner $68 \%$ of the distribution, see appendix B) of $7.82 \mathrm{~m}$. This is shown figure 4.9 of the empirical CDF plot of the differences (in blue) and the fitted distribution (in red). 
Previously the estimated error in the DEM was calculated to have standard deviation of $\sigma_{D E M}=7.57 \mathrm{~m}$. Since the distribution of the differences has a standard deviation of 7.82 , the standard deviation of the error in the height code 1 elevations can be calculated as $\sigma_{1}=\sqrt{7.82^{2}-7.57^{2}} \approx 1.96 \mathrm{~m}$ to two decimal places.

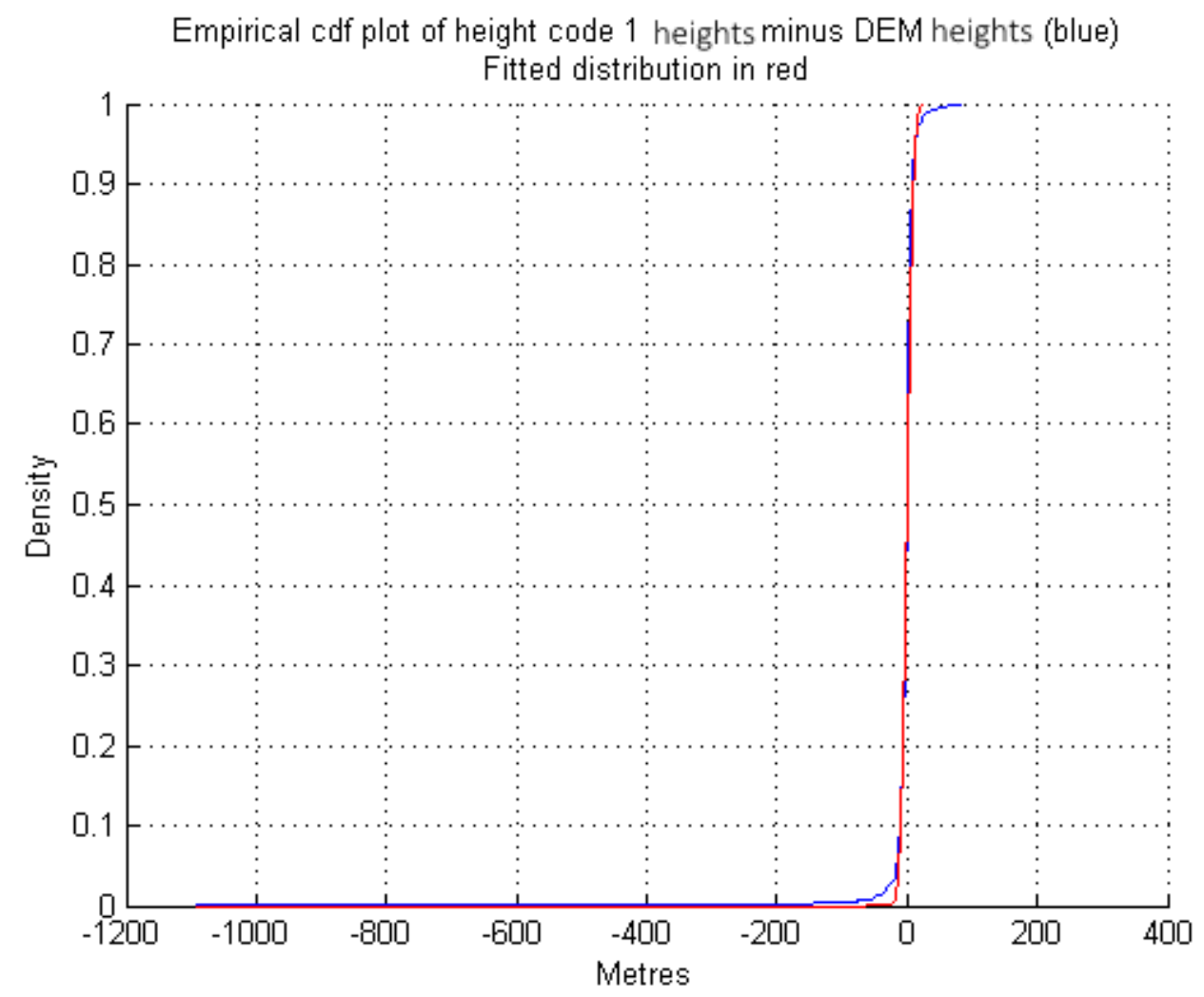

Figure 4.9: Empirical CDF of the heights with code 1 minus heights derived from the 8 $\mathrm{m}$ DEM (in blue) and distribution fitted to the inner 68\% (in red).

The distribution shown in figure 4.9 has long tails. Height differences on these tails most likely correspond to data points which have been incorrectly located horizontally or the spot height has been estimated poorly. For these data points the estimated $1.96 \mathrm{~m}$ error in height is too small and so more appropriate standard deviation have been calculated for these points as follows. The Gaussian CDF function can be written as,

$$
F(x)=\frac{1}{2}\left(1+\operatorname{erf}\left(\frac{x}{\sqrt{2} \sigma}\right)\right) .
$$

For all the height differences $x$ in the distribution greater than $2 \sigma_{1}$ in absolute value, a revised standard deviation $\tilde{\sigma_{1}}$ has been calculated by,

$$
\tilde{\sigma}_{1}=\sqrt{\left(\frac{x}{\sqrt{2} e r f^{-1}(2 \tilde{F}(x)-1)}\right)^{2}-7.57^{2}}
$$


where $\tilde{F}(x)$ is drawn from the empirical CDF. Finally, heights have also been estimated for the locations with a height code 2 from the DEM. The difference between recorded heights and the DEM heights for locations with a height code of 2 (i.e. barometric heights) has a standard deviation ( fitted to the inner $68 \%$ of the distribution) of 11.84 $\mathrm{m}$. This is shown in figure 4.10 of the empirical CDF plot of the differences (in blue) and the fitted distribution (in red). Since the distribution of the differences has a standard deviation of 7.8201, the standard deviation of the error in the height code 2 elevations can be calculated as $\sigma_{2}=\sqrt{11.84^{2}-7.57^{2}} \approx 9.10 \mathrm{~m}$ to two decimal places.

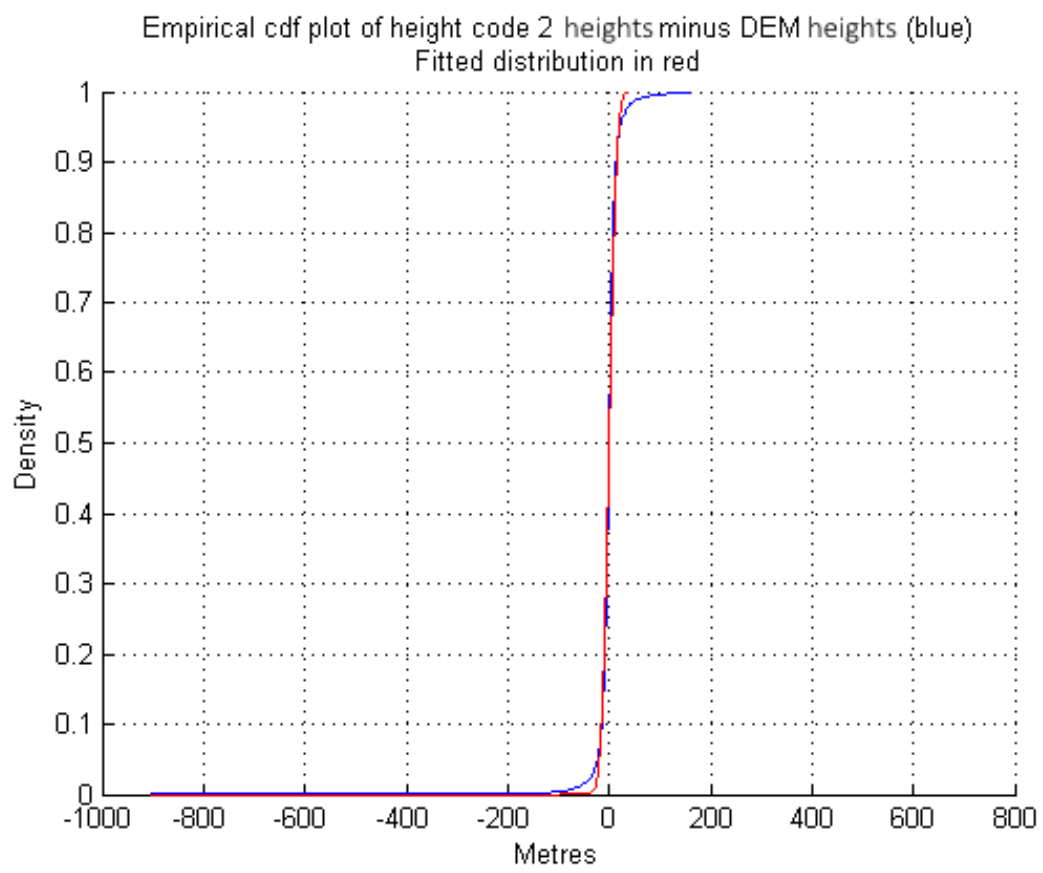

Figure 4.10: Empirical CDF of the heights with code 2 minus heights derived from the 8 $\mathrm{m}$ DEM (in blue) and distribution fitted to the inner 68\% (in red).

Again, the distribution shown in figure 4.10 has long tails (outside the $2 \sigma_{2}$ range). Height differences on these tails most likely correspond to data points where the barometric height has been estimated poorly. For these data points the estimated $9 \mathrm{~m}$ error in height is too small and so more appropriate height error standard deviations have been calculated, as before.

Figure 4.11 shows the estimated standard deviations of the heights of the gravity observations in the GNS Science terrestrial gravity database gridded at a 1 arc minute resolution. Predominantly the standard deviations are around $9 \mathrm{~m}$ with sparsely located larger values (which were the points on the tails of the height code 1 and 2 DEM height differences) which are typically isolated to individual points.

During the gravity reductions the heights, $H_{i}$, were used when performing the free air 


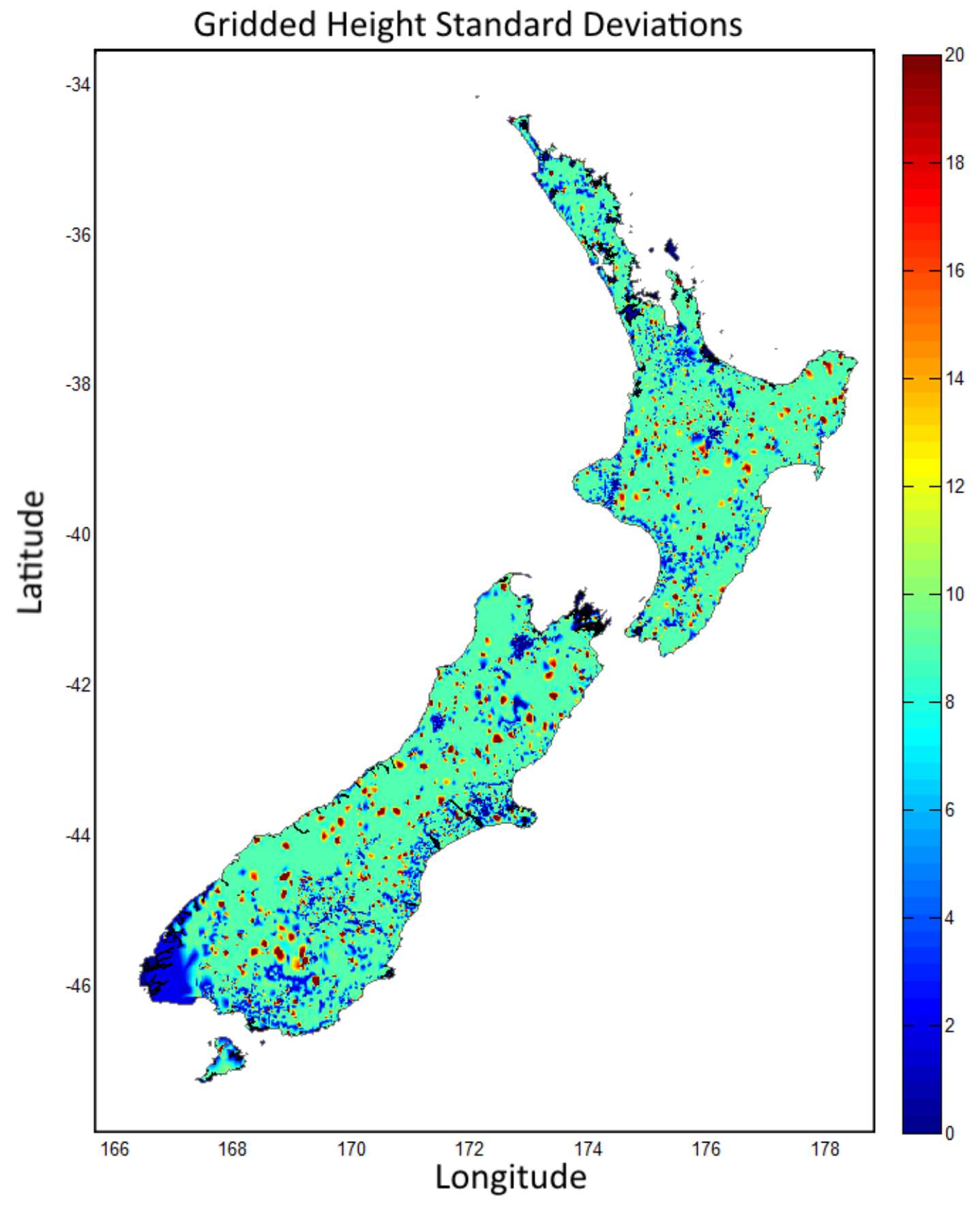

Figure 4.11: Derived height standard deviations (in metres) for each gravity observation in the GNS Science terrestrial gravity database.

correction $\left(\approx 0.3086 H_{i}(\mathrm{mGal})\right)$ and the Bouguer slab correction $\left(-0.0419 \rho H_{i} \mathrm{mGal}\right)$. For this reason the estimated variances of the noise level, $N_{i}$ for each gravity observation has been calculated as $N_{i}=\left((0.3086-0.0419 \rho) \sigma_{i}\right)^{2}$.

Figure 4.12 shows the estimated standard deviation (in $\mathrm{mGal}$ ) for each of the gravity 
anomalies gridded at 1 arc minute using a linear interpolation algorithm. In general the estimated error is approximately $3 \mathrm{mGal}$ with concentrated patches of $0.5 \mathrm{mGal}$ and 0.02 $\mathrm{mGal}$ where heights have code 0 and larger values where the heights have been estimated to be particularly poor.

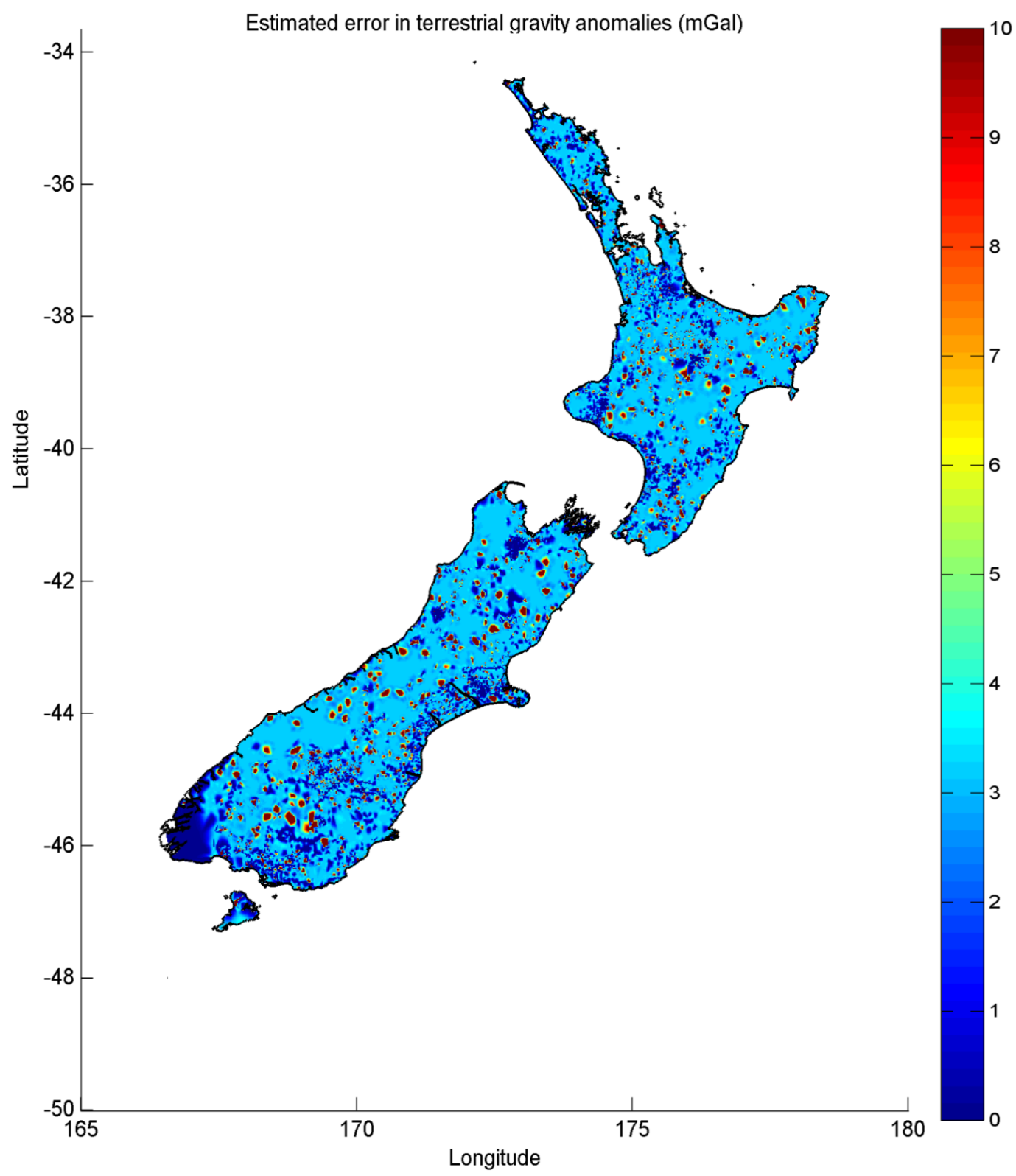

Figure 4.12: Linearly gridded estimated error of the terrestrial gravity observations in mGal.

These estimated errors will be used to down weight the effect of outliers when the data is gridded using least squares collocation (see chapter 7). 


\subsection{Summary}

This chapter gives an overview of the terrestrial gravity observations available in the GNS Science terrestrial gravity database. The density of the measurements has been discussed and displayed over the North Island, South Island and Stewart Island. Additionally the data covering Chatham and Pitt Islands have been shown.

The gravitational effect of normal gravity and the topography have been re-calculated, despite being provided in the database. This was done to ensure consistency in the data reductions due to the historic calculations and conversion formulas used to derive similar corrections in the database. The optimal terrain correction was shown to be a composite correction of both the terrain corrections calculated from the $8 \mathrm{~m}$ digital elevation model and field observations, since it best reduced any errors arising from mis-locating the terrestrial gravity sites.

An assessment of the discrepancies in the recorded vertical positions of the gravity observation sites was also discussed. Discrepancies were determined by comparing recorded heights to those derived from the $8 \mathrm{~m}$ DEM following Stagpoole (2012) and they were used to estimate the propagated error in the free air and Bouguer slab gravity corrections. 


\section{Chapter 5}

\section{Global Gravity Models (GGM)}

\subsection{Introduction}

This chapter gives a description of global gravity models (GGM) and how they are used to approximate the disturbing potential gravity field/ geoid height anomalies and gravity disturbances up to some maximum spherical harmonic degree and order.

Following Amos and Featherstone (2003), thirty seven satellite only global gravity models and nine combined global gravity models are compared against terrestrial gravity data and leveling data derived height anomalies. Of the thirty seven satellite only models, GO_CONS_GCF_2_DIR_R5 provides the best fit to the terrestrial gravity and leveling derived height anomalies data sets.

For the nine combined models, EGM2008 fits the terrestrial gravity data best and EIGEN6C4 best fits the leveling data derived height anomalies, this is similar to the result obtained by Huang, J. and Veronneau, M. (2015). The difference between the two models is discussed in detail.

\subsection{Spherical harmonic coefficients}

Global gravity models (GGM) consist of a set of spherical harmonic coefficients $\left(\delta \bar{C}_{n m}\right.$ and $\left.\bar{S}_{n m}\right)$ and standard deviations of the harmonic coefficients $\left(q_{\bar{C}_{n m}}\right.$ and $\left.q_{\bar{S}_{n m}}\right)$ up to some maximum degree and order $M$ (Amos, 2007).

The disturbing potential $T_{M}$ is approximated from coefficients by the spherical harmonic expansion,

$$
T_{M}=\frac{G M}{r} \sum_{n=2}^{M}\left(\frac{a}{r}\right)^{n} \sum_{m=0}^{n}\left(\delta \bar{C}_{n m} \cos m \lambda+\bar{S}_{n m} \sin m \lambda\right) \bar{P}_{n m}(\cos \theta),
$$

$\bar{P}_{n m}(\cos \theta)$ are fully normalized associated Legendre functions and $\delta \bar{C}_{n m}$ and $\bar{S}_{n m}$ are fully normalized spherical harmonic coefficients which have been reduced for the zonal harmonics of the reference ellipsoid. $a$ is the average radius of the earth and $(r, \theta, \lambda)$ is an arbitrary point in space in geocentric spherical polar coordinates. 
For a given value of $M$, the minimum wavelength of the expansion is approximated by the following,

$$
L_{\max }=\frac{2 \pi R}{M} \cos \phi
$$

and resolution is given by

$$
\rho_{\max }=\frac{\pi R}{M} \cos \phi
$$

at some latitude $\phi$ and Earth radius $R$. e.g if $M=2160$ and $R=6378.137 \mathrm{~km}$ then the minimum wavelength at $\phi=45^{\circ}$ is approximately $9 \mathrm{~km}$.

By Bruns' equation, the geoid height anomaly is given by,

$$
\xi_{M}=\frac{G M}{r \gamma} \sum_{n=2}^{M}\left(\frac{a}{r}\right)^{n} \sum_{m=0}^{n}\left(\delta \bar{C}_{n m} \cos m \lambda+\bar{S}_{n m} \sin m \lambda\right) \bar{P}_{n m}(\cos \theta),
$$

and the gravity anomaly is given by the radial derivative,

$$
\Delta g_{M}=\frac{G M}{r^{2}} \sum_{n=2}^{M}\left(\frac{a}{r}\right)^{n}(n-1) \sum_{m=0}^{n}\left(\delta \bar{C}_{n m} \cos m \lambda+\bar{S}_{n m} \sin m \lambda\right) \bar{P}_{n m}(\cos \theta) .
$$

The following equation is used to calculate the error degree variances $\epsilon^{2}$ for gravity anomalies. It is used to assess the accuracy of global gravity model coefficients for degree $n$ in predicting the $n$-th spherical harmonic of the gravity field.

$$
\epsilon^{2}(n)=\left(\frac{G M}{a^{2}}\right)^{2}(n-1)^{2} \sum_{m=0}^{n} q_{\bar{C}_{n m}}^{2}+q_{\bar{S}_{n m}}^{2} .
$$

To determine the most accurate global gravity model of a given set, it is useful to compare computed values of $\epsilon^{2}$ between models. It is also useful to compare derived values of $\Delta g$ and $\xi$ to ground based gravity free air anomaly data and leveling derived height anomalies.

Global gravity models come in two main forms, satellite only and combined. The satellite only models are determined solely from satellite gravity field measurements. Typically, satellites can only detect long wavelengths in the gravity field (in part due to their elevation above the earth causing severe attenuation of short wavelength gravity signal as discussed in section 2.3). For this reason the models only contain coefficients $\delta \bar{C}_{n m}$ and $\bar{S}_{n m}$ up to a maximum degree $M \approx 300$.

Combined global gravity models are determined from multiple data sources, namely satellite gravity observations supplemented with ground based gravity data, satellite altimetry measurements and occasionally shipborne and airborne data. The additional data sets contain higher frequency gravity signals which means higher degree coefficients in the global gravity model can be determined. These models often include coefficients up to maximum degree and order $M=1500$ and higher, as can be seen in table 5.2. 


\subsection{GGM comparison}

Gravity anomalies $\Delta g$ and geoid heights $\xi$ have been determined for 37 satellite only gravity models and 9 combined models. These data have been compared to 40667 New Zealand terrestrial gravity derived free air anomalies (as discussed in section 4.3) and 1442 leveling derived geoid heights. The two types of global gravity models are compared separately. The following two tables show the satellite only and combined global gravity models compared here.

Table 5.1: Satellite only global gravity models compared.

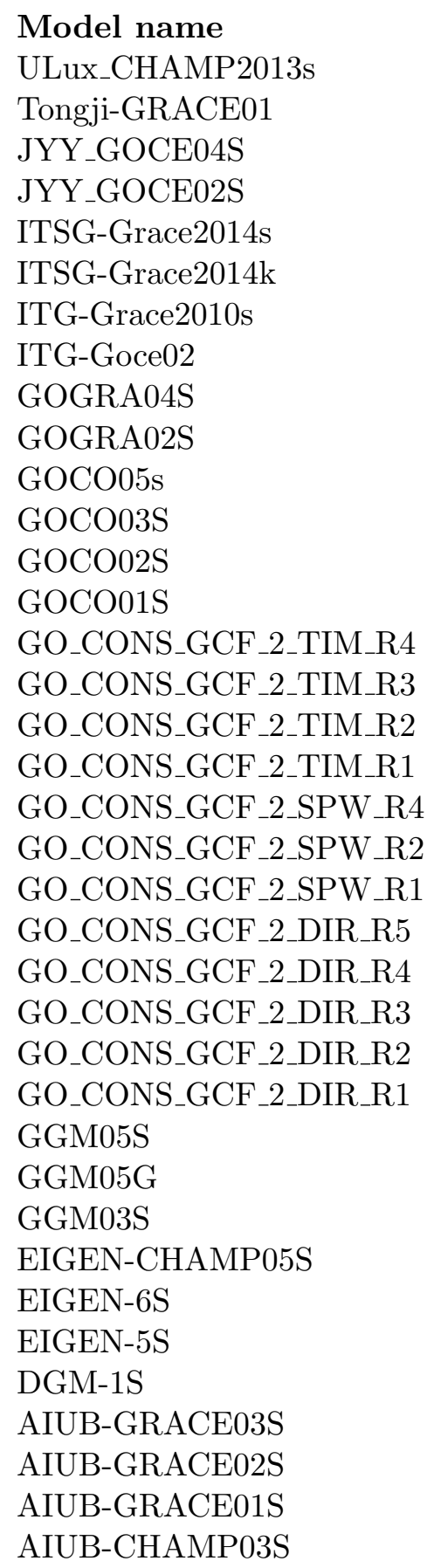

$\begin{array}{lll}\text { Year } & \text { Max Degree } & \text { Satellite data sources, S( ) } \\ 2013 & 120 & \text { S(Champ) } \\ 2013 & 160 & \text { S(Grace) } \\ 2014 & 230 & \text { S(Goce) } \\ 2013 & 230 & \text { S(Goce) } \\ 2014 & 200 & \text { S(Grace) } \\ 2014 & 200 & \text { S(Grace) } \\ 2010 & 180 & \text { S(Grace) } \\ 2013 & 240 & \text { S(Goce) } \\ 2014 & 230 & \text { S(Goce,Grace) } \\ 2013 & 230 & \text { S(Goce,Grace) } \\ 2015 & 280 & \text { S(GOCE,GRACE,CHAMP) } \\ 2012 & 250 & \text { S(Goce,Grace) } \\ 2011 & 250 & \text { S(Goce,Grace) } \\ 2010 & 224 & \text { S(Goce,Grace) } \\ 2013 & 250 & \text { S(Goce) } \\ 2011 & 250 & \text { S(Goce) } \\ 2011 & 250 & \text { S(Goce) } \\ 2010 & 224 & \text { S(Goce) } \\ 2014 & 280 & \text { S(Goce) } \\ 2011 & 240 & \text { S(Goce) } \\ 2010 & 210 & \text { S(Goce) } \\ 2014 & 300 & \text { S(Goce,Grace,Lageos) } \\ 2013 & 260 & \text { S(Goce,Grace,Lageos) } \\ 2011 & 240 & \text { S(Goce,Grace,Lageos) } \\ 2011 & 240 & \text { S(Goce) } \\ 2010 & 240 & \text { S(Goce) } \\ 2014 & 180 & \text { S(Grace) } \\ 2015 & 240 & \text { S(Grace,Goce) } \\ 2008 & 180 & \text { S(Grace) } \\ 2010 & 150 & \text { S(Champ) } \\ 2011 & 240 & \text { S(Goce,Grace,Lageos) } \\ 2008 & 150 & \text { S(Grace,Lageos) } \\ 2012 & 250 & \text { S(Goce,Grace) } \\ 2011 & 160 & \text { S(Grace) } \\ 2009 & 150 & \text { S(Grace) } \\ 2008 & 120 & \text { S(Grace) } \\ 2010 & 100 & \text { S(Champ) } \\ & & \end{array}$


Table 5.2: Combined global gravity models compared

\begin{tabular}{|c|c|c|c|}
\hline Model name & Year & Max Degree & $\begin{array}{l}\text { Data sources, } \mathrm{S}() \text { satellite } \\
\mathrm{G} \text { terrestrial gravity data, } \\
\text { A altimetry data }\end{array}$ \\
\hline EGM2008 & 2008 & 2190 & $\mathrm{~S}($ Grace $), \mathrm{G}, \mathrm{A}$ \\
\hline EIGEN-51C & 2010 & 359 & S(Grace,Champ), G,A \\
\hline EIGEN-5C & 2008 & 360 & S(Grace,Lageos),G,A \\
\hline EIGEN-6C & 2011 & 1420 & S(Goce,Grace,Lageos), G,A \\
\hline EIGEN-6C2 & 2012 & 1949 & S(Goce,Grace,Lageos),G,A \\
\hline EIGEN-6C3stat & 2014 & 1949 & $\mathrm{~S}$ (Goce - dir 4, Grace,Lageos),G,A \\
\hline EIGEN-6C4 & 2014 & 2190 & S(Goce dir 5,Grace,Lageos), G,A \\
\hline GGM03C & 2009 & 360 & $\mathrm{~S}($ Grace $), \mathrm{G}, \mathrm{A}$ \\
\hline GIF48 & 2011 & 360 & $\mathrm{~S}$ (Grace), G,A \\
\hline
\end{tabular}

All of the models here have been evaluated up to their maximum degree and order. The coefficients are freely available from http://icgem.gfz-potsdam.de/ICGEM/modelstab.html, (at the time of writing, 2015).

The calculated values of $\Delta g$ and $\xi$ for each model have been subtracted from the terrestrial free air anomalies and leveling derived height anomalies. The root mean squared residual, standard deviation (computed from the inner $68 \%$ of each distribution since the distributions are generally leptokurtotic i.e. kurtosis $>3$, see appendix B), min, max and kurtosis of the difference has been given in each case.

\subsubsection{Satellite only global gravity models:}

Of the satellite only models, GO_CONS_GCF _2_DIR_R5 (Bruinsma et al, 2013) provided the best fit to the terrestrial free air anomaly data and the leveling derived height anomalies. This is shown in tables 5.3 and 5.4. Moreover GO_CONS_GCF_2_DIR_R5 contains coefficients up to the highest maximum degree and order and overall it has the smallest error degree variances beyond degree and order 130 and has a negligible difference in accuracy for lower degrees in comparison to other satellite only coefficients. This can be seen in figure 5.1.

5th Generation GOCE model ( GO_CONS_GCF_2_DIR_R5): The fith generation GOCE gravity model (model name GO_CONS_GCF_2_DIR_R5) has a maximum degree and order of 300 and was computed from GOCE-SGG with GRACE and LAGEOS satellite data sets. The GOCE data used for the model were collected over a period of approximately 1259 days and 19380 orbital revolutions. The GRACE data have a measurement period of approximately 10 years (2003 - 2012) and its contribution in the model was taken only up to degree and order 130. The LAGEOS-1 and -2 data have been collected over approximately a 25 year period (1985 - 2011) and were used in the model for the very low degrees (2 and 3) since these cannot be estimated accurately by the GRACE and GOCE data. 
Table 5.3: Table of statistics of the terrestrial gravity free air anomalies at 40667 locations minus $\Delta g$ computed from the 37 satellite only global gravity models in mGals.

\begin{tabular}{|c|c|c|c|c|c|}
\hline Model name & RMS & STD & Min & Max & Kurtosis \\
\hline GO_CONS_GCF_2_DIR_R5 & 37.6073 & 22.1671 & -93.0868 & 112.9472 & 4.9775 \\
\hline GGM05S & 36.264 & 22.1813 & -74.4339 & 110.1558 & 3.5592 \\
\hline GOCO05s & 37.575 & 22.1867 & -93.2082 & 112.6967 & 4.8659 \\
\hline AIUB-CHAMP03S & 30.1996 & 22.5748 & -88.7753 & 62.012 & 4.1235 \\
\hline GO_CONS_GCF_2_DIR_R4 & 36.8304 & 22.7331 & -77.3117 & 113.1452 & 4.2265 \\
\hline GOCO01S & 38.0639 & 22.8123 & -80.1339 & 94.705 & 3.6377 \\
\hline GO_CONS_GCF_2_SPW_R4 & 37.4639 & 22.966 & -84.9444 & 122.9355 & 4.5249 \\
\hline GO_CONS_GCF_2_DIR_R1 & 38.1742 & 23.003 & -84.4961 & 114.2464 & 4.2723 \\
\hline GO_CONS_GCF_2_DIR_R3 & 37.6143 & 23.2482 & -75.2231 & 103.2209 & 3.757 \\
\hline GO_CONS_GCF_2_TIM_R4 & 37.7004 & 23.3532 & -81.8812 & 115.7305 & 4.1983 \\
\hline GO_CONS_GCF_2_TIM_R1 & 38.6305 & 23.3743 & -82.5647 & 94.1266 & 3.6444 \\
\hline GOCO03S & 37.4925 & 23.4205 & -75.2881 & 106.6389 & 3.9148 \\
\hline GOCO02S & 37.4944 & 23.4334 & -74.8143 & 102.7886 & 3.9584 \\
\hline GOGRA02S & 38.1268 & 23.6293 & -84.1123 & 113.6498 & 4.1554 \\
\hline JYY_GOCE02S & 38.1267 & 23.6316 & -84.1164 & 113.5754 & 4.1579 \\
\hline GO_CONS_GCF_2_TIM_R2 & 37.7021 & 23.673 & -76.011 & 103.2832 & 3.9698 \\
\hline GGM05G & 38.0033 & 23.6948 & -76.8299 & 119.0587 & 3.9467 \\
\hline GOGRA04S & 38.0151 & 23.9338 & -76.287 & 109.2225 & 3.9362 \\
\hline JYY_GOCE04S & 38.0287 & 23.9368 & -76.317 & 108.9305 & 3.9349 \\
\hline ITG-Goce02 & 38.0438 & 23.9536 & -73.0812 & 104.8443 & 3.7965 \\
\hline GO_CONS_GCF_2_TIM_R3 & 37.719 & 23.959 & -76.3918 & 108.2268 & 3.9504 \\
\hline DGM-1S & 37.4792 & 23.9628 & -73.8347 & 106.5596 & 3.7326 \\
\hline EIGEN-6S & 37.6672 & 24.0817 & -87.2624 & 110.3405 & 3.9528 \\
\hline GO_CONS_GCF_2_DIR_R2 & 38.1105 & 24.4199 & -86.811 & 110.3547 & 3.8868 \\
\hline AIUB-GRACE01S & 37.042 & 24.5684 & -96.7775 & 83.9338 & 3.7917 \\
\hline GO_CONS_GCF_2_SPW_R2 & 38.3276 & 25.0336 & -72.3152 & 94.9571 & 3.2665 \\
\hline ITG-Grace2010s & 38.8598 & 25.3639 & -61.8203 & 93.8103 & 2.9013 \\
\hline EIGEN-CHAMP05S & 29.4033 & 25.8592 & -79.801 & 57.1033 & 3.2923 \\
\hline ITSG-Grace2014k & 39.3789 & 25.9488 & -67.9165 & 91.9359 & 3.0217 \\
\hline GO_CONS_GCF_2_SPW_R1 & 38.8685 & 26.2716 & -82.5727 & 88.9577 & 3.3558 \\
\hline Tongji-GRACE01 & 38.933 & 26.703 & -75.0992 & 84.0589 & 2.9155 \\
\hline EIGEN-5S & 38.3322 & 26.8785 & -77.6046 & 84.5698 & 2.8317 \\
\hline AIUB-GRACE03S & 39.394 & 27.3156 & -75.4189 & 85.9106 & 2.9971 \\
\hline AIUB-GRACE02S & 40.7018 & 27.7031 & -97.4028 & 83.4522 & 3.1711 \\
\hline ULux_CHAMP2013s & 34.0017 & 28.7899 & -84.1968 & 69.7918 & 2.6834 \\
\hline GGM03S & 48.0025 & 38.6618 & -132.2975 & 122.9492 & 3.126 \\
\hline ITSG-Grace2014s & 50.7239 & 46.2059 & -118.2534 & 108.1328 & 2.4802 \\
\hline
\end{tabular}


Table 5.4: Table of statistics of the leveling derived height anomalies at 1442 locations minus $\xi$ computed from the 37 satellite only global gravity models. The residual for each of the 13 local vertical datums has been demeaned for each model as discussed in section 3.4 i.e. to remove any local vertical datum offsets. Values are in metres.

\begin{tabular}{|c|c|c|c|c|c|}
\hline Model & RMS & STD & Min & Max & Kurtosis \\
\hline GO_CONS_GCF_2_DIR_R5 & 0.2959 & 0.2960 & -1.1363 & 0.855 & 3.7597 \\
\hline GOCO05s & 0.301 & 0.3011 & -1.1587 & 0.9008 & 3.5757 \\
\hline GO_CONS_GCF_2_SPW_R4 & 0.3117 & 0.3118 & -1.1016 & 0.949 & 3.5946 \\
\hline GO_CONS_GCF_2_DIR_R4 & 0.3286 & 0.3287 & -1.1559 & 1.1581 & 4.0621 \\
\hline GO_CONS_GCF_2_TIM_R4 & 0.3295 & 0.3296 & -1.0786 & 1.1142 & 3.7335 \\
\hline GO_CONS_GCF_2_DIR_R1 & 0.3377 & 0.3378 & -1.2008 & 1.0258 & 3.5649 \\
\hline GO_CONS_GCF_2_TIM_R3 & 0.3418 & 0.3419 & -1.1428 & 1.2087 & 3.9984 \\
\hline GOCO03S & 0.3514 & 0.3515 & -1.1817 & 1.2319 & 4.2393 \\
\hline GO_CONS_GCF_2_TIM_R2 & 0.353 & 0.3531 & -1.1378 & 1.2104 & 3.9305 \\
\hline GOCO02S & 0.3565 & 0.3566 & -1.1405 & 1.2214 & 4.0095 \\
\hline JYY_GOCE02S & 0.3577 & 0.3579 & -1.2084 & 1.2988 & 3.6636 \\
\hline GOGRA02S & 0.3583 & 0.3584 & -1.1995 & 1.3021 & 3.6664 \\
\hline GGM05G & 0.3592 & 0.3593 & -1.2541 & 1.0764 & 3.7223 \\
\hline JYY_GOCE04S & 0.364 & 0.3641 & -1.2793 & 1.2682 & 4.1168 \\
\hline GOGRA04S & 0.3648 & 0.3649 & -1.2716 & 1.2702 & 4.1052 \\
\hline ITG-Goce02 & 0.3655 & 0.3656 & -1.2086 & 1.2747 & 4.0581 \\
\hline DGM-1S & 0.3678 & 0.3679 & -1.164 & 1.3102 & 4.1792 \\
\hline GO_CONS_GCF_2_DIR_R2 & 0.369 & 0.3692 & -1.2274 & 1.2604 & 3.6315 \\
\hline GO_CONS_GCF_2_DIR_R3 & 0.3714 & 0.3716 & -1.3773 & 1.3315 & 4.5353 \\
\hline EIGEN-6S & 0.3759 & 0.376 & -1.217 & 1.2407 & 3.4455 \\
\hline GOCO01S & 0.4608 & 0.461 & -1.4504 & 1.714 & 4.6961 \\
\hline GO_CONS_GCF_2_TIM_R1 & 0.4611 & 0.4613 & -1.436 & 1.7393 & 4.753 \\
\hline GO_CONS_GCF_2_SPW_R2 & 0.4645 & 0.4646 & -1.8544 & 2.0984 & 4.9671 \\
\hline GO_CONS_GCF_2_SPW_R1 & 0.5045 & 0.5047 & -1.9645 & 2.0774 & 4.8493 \\
\hline ITG-Grace2010s & 0.608 & 0.6082 & -2.3908 & 2.8321 & 5.9777 \\
\hline ITSG-Grace2014k & 0.6125 & 0.6127 & -2.3545 & 3.0439 & 5.8506 \\
\hline GGM05S & 0.6558 & 0.6561 & -2.1962 & 2.8913 & 4.2781 \\
\hline Tongji-GRACE01 & 0.7092 & 0.7095 & -2.5449 & 3.863 & 7.9658 \\
\hline AIUB-GRACE02S & 0.7518 & 0.7521 & -3.1222 & 4.1126 & 6.9664 \\
\hline AIUB-GRACE03S & 0.7549 & 0.7552 & -2.6117 & 4.0152 & 7.6883 \\
\hline EIGEN-5S & 0.7569 & 0.7572 & -2.903 & 4.0912 & 8.2753 \\
\hline AIUB-GRACE01S & 0.8644 & 0.8647 & -4.653 & 4.8502 & 9.3208 \\
\hline AIUB-CHAMP03S & 0.9309 & 0.9312 & -4.9137 & 5.0933 & 11.1112 \\
\hline EIGEN-CHAMP05S & 0.9397 & 0.9401 & -3.7561 & 4.896 & 9.4562 \\
\hline ITSG-Grace2014s & 0.9699 & 0.9703 & -3.6884 & 3.1677 & 3.6093 \\
\hline ULux_CHAMP2013s & 1.0032 & 1.0036 & -3.1938 & 4.4255 & 6.1044 \\
\hline GGM03S & 1.1046 & 1.105 & -4.6427 & 2.8927 & 3.8498 \\
\hline
\end{tabular}




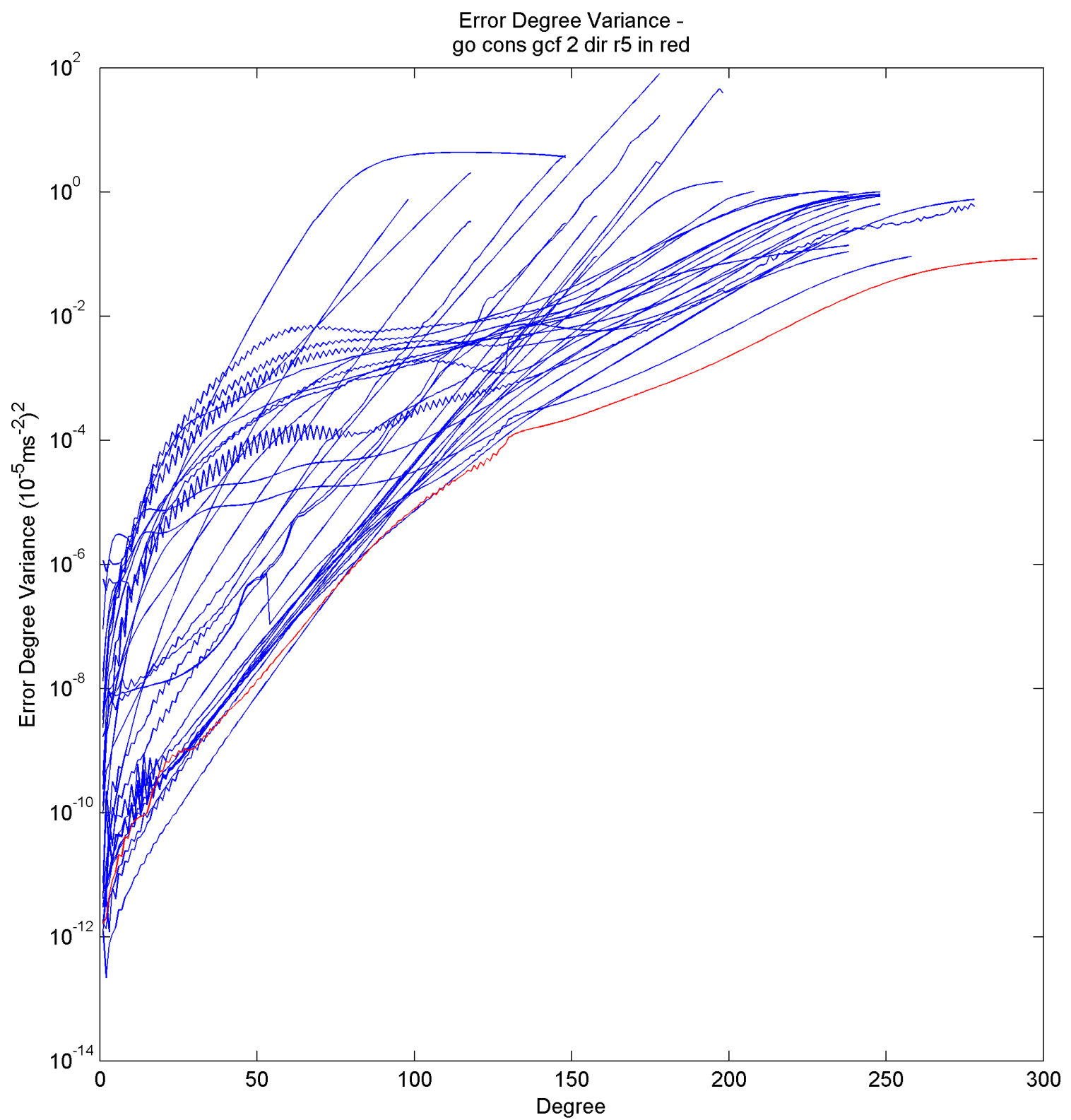

Figure 5.1: Error degree variance plots for all satellite only models with GO_CONS_GCF_2_DIR_R5 in red.

\subsubsection{Combined global gravity models.}

Of the nine combined global gravity models, two particular models give favourable fits. EGM2008 (Pavlis et al, 2008) fits the terrestrial gravity data the best whilst EIGEN-6C4 (Forste et al, 2015) gives the best fit to the leveling derived height anomalies. Both models contain coefficients up to degree 2190. These optimal fits are shown by the standard deviations in tables 5.5 and 5.6. 
Table 5.5: Table of statistics of the terrestrial gravity free air anomalies at 40667 locations minus $\Delta g$ computed from the 9 combined global gravity models in mGals.

$\begin{array}{llllll}\text { Name } & \text { RMS } & \text { STD } & \text { Min } & \text { Max } & \text { Kurtosis } \\ \text { EGM2008 } & \mathbf{1 7 . 8 9 5} & \mathbf{1 0 . 7 5 9 5} & -93.3966 & 153.8344 & 9.839 \\ \text { EIGEN-6C4 } & \mathbf{1 8 . 5 2 4 4} & \mathbf{1 1 . 2 7 5 7} & -91.1377 & 158.6221 & 9.7456 \\ \text { EIGEN-6C3stat } & 19.1893 & 11.8747 & -93.276 & 156.2887 & 9.2259 \\ \text { EIGEN-6C2 } & 19.7802 & 12.901 & -92.5833 & 158.429 & 8.7311 \\ \text { EIGEN-6C } & 23.0343 & 15.7583 & -95.6303 & 146.6954 & 7.3267 \\ \text { GIF48 } & 31.6523 & 25.1956 & -130.2008 & 128.2044 & 4.4832 \\ \text { EIGEN-51C } & 32.7893 & 27.7791 & -132.5116 & 135.7618 & 3.9617 \\ \text { GGM03C } & 33.6504 & 27.8076 & -148.9071 & 127.9324 & 4.1237 \\ \text { EIGEN-5C } & 34.2565 & 29.5735 & -152.7708 & 112.7076 & 3.6278\end{array}$

Table 5.6: Table of statistics of the leveling derived height anomalies at 1442 locations minus $\xi$ computed from the 9 combined global gravity models. The residual for each of the 13 local vertical datums has been demeaned for each model as discussed in section 3.4 i.e. to remove any local vertical datum offsets. Values are in metres.

$\begin{array}{llllll}\text { Name } & \text { RMS } & \text { STD } & \text { Min } & \text { Max } & \text { Kurtosis } \\ \text { EIGEN-6C4 } & \mathbf{0 . 0 5 2 9 1 3} & \mathbf{0 . 0 4 5 6 9 5} & -0.32938 & 0.29049 & 5.9625 \\ \text { EIGEN-6C2 } & 0.053179 & 0.044851 & -0.33072 & 0.27795 & 5.9508 \\ \text { EIGEN-6C3stat } & 0.053506 & 0.04537 & -0.34567 & 0.2608 & 5.8171 \\ \text { EGM2008 } & \mathbf{0 . 0 6 3 3 4 9} & \mathbf{0 . 0 5 1 9 1} & -0.33815 & 0.28139 & 4.9277 \\ \text { EIGEN-6C } & 0.062464 & 0.054001 & -0.38508 & 0.2568 & 4.5999 \\ \text { EIGEN-51C } & 0.20066 & 0.18726 & -0.64107 & 0.94671 & 4.5778 \\ \text { GIF48 } & 0.20864 & 0.19757 & -0.68574 & 0.82133 & 4.0082 \\ \text { GGM03C } & 0.3073 & 0.27562 & -1.3577 & 0.98843 & 4.2713 \\ \text { EIGEN-5C } & 0.32364 & 0.2944 & -1.6636 & 1.4704 & 4.5849\end{array}$

EGM2008: EGM2008 is a high resolution gravity model which was released by the National Geospatial-Intelligence Agency (NGA) in 2008. EGM2008 is complete to degree and order 2159 (i.e. it has coefficients $\bar{C}_{n m}$ and $\bar{S}_{n m}$ up to $M=2159$ ) and contains coefficients up to degree 2190 where as its predecessor, EGM96 only contained spherical harmonic coefficients up to degree and order 360. This is an improvement in resolution of around $24 \mathrm{~km}$ at latitudes of 45 degrees. The model coefficients are freely available from http://earth-info.nima.mil/GandG/.

The model was computed from a $5^{\prime} \times 5^{\prime}$ global gravity database (from land and satellite altimetry sources) and (at the time of computation) the latest GRACE - based satellite gravity data for low order spherical harmonic coefficients. Over New Zealand, gridded terrestrial gravity data were supplied for the model from the GNS Science terrestrial gravity database; this data set is described in detail in section 4.1.

The accuracy of EGM2008 has been evaluated by several authors. Gruber (2009) compared the EGM2008, EGM96 and other satellite gravity models to independent GPS- 
leveling heights from Australia (197 points), Germany (675 points ), Europe (1233 points), Canada (430 points), Japan (837 points) and USA (5168 points). The accuracy of EGM2008 varied, from $3.8 \mathrm{~cm}$ (for Germany) to $33.4 \mathrm{~cm}$ (USA), but was consistently better than the EGM96 model over all maximum degrees $M$.

The $3 \mathrm{~cm}$ accuracy in Germany is confirmed in Hirt (2012) and the high accuracy is attributed to the dense gravity data sets which were used for the model's development. Claessens et al (2009b) compared EGM2008 to 254 GPS-leveling data and gravity observations across Australia. They found the standard deviation of the difference between the GGM and the leveling data to be $17.3 \mathrm{~cm}$ and $5.5 \mathrm{mGal}$ for the free air anomaly data. This is better than twice the accuracy of EGM96 which had a disagreement between the GGM and leveling data set of $33.4 \mathrm{~cm}$ and $11.7 \mathrm{mGal}$ for the free air anomalies.

EIGEN-6C4 is also a high resolution global gravity model which was released in 2014 and similarly is complete to degree and order 2159 (i.e. it has coefficients $\bar{C}_{n m}$ and $\bar{S}_{n m}$ up to $M=2159$ ) and contains coefficient up to degree 2190. It was computed from LAGEOS-1/2, the latest GRACE and GO_CONS_GCF_2_DIR_R5 satellite gravity data and surface gravity data from a 2'x2' global gravity anomaly grid which consists of altimetry data over the oceans and EGM2008 over continents. The combination of the data sets with respect to varying degrees is shown in the following figure.

\section{Combination scheme of the normal equations for EIGEN-6C4}

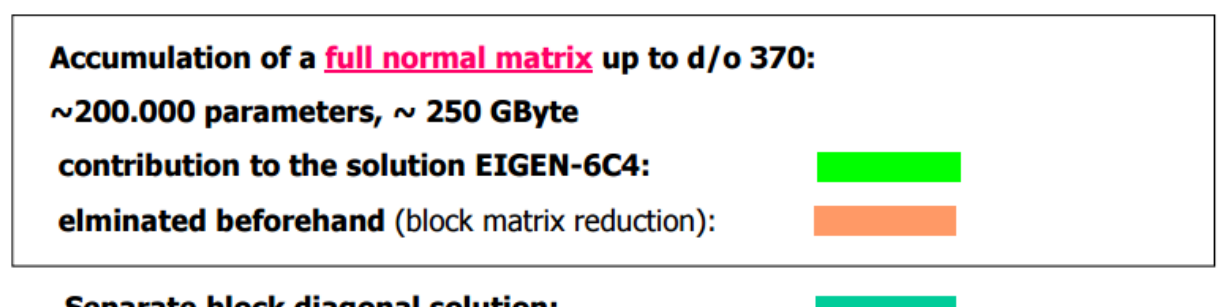

Separate block diagonal solution:

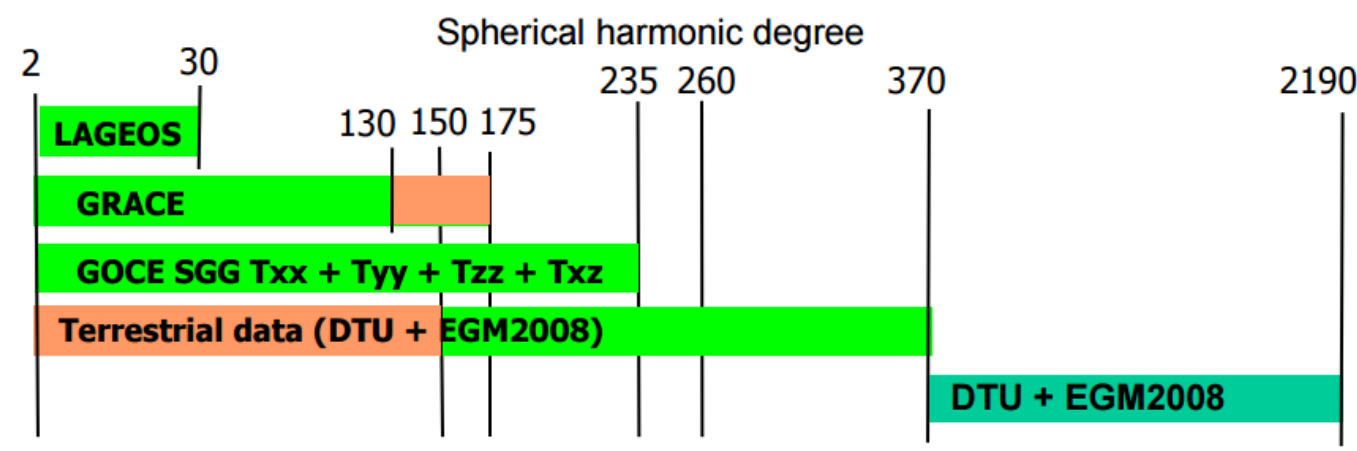

Figure 5.2: Combination scheme with respect to model degree of data sets used to compute EIGEN-6C4. Adapted from slide 4 of Forste et al (2014) 
Figure 5.2 demonstrates the key difference between EGM2008 and EIGEN-6C4 is that just satellite gravity data is used to derive the long wavelength coefficients up to degree 150 for EIGEN-6C4. These satellite sources include the GO_CONS_GCF_2_DIR_R5 model which was determined to be superior over other satellite only models in section 5.3.1, and new GRACE data, whereas, the longer wavelength coefficients derived for EGM2008 were based on older GRACE data and a 5'x5' grid of surface gravity data. Additionally EIGEN-6C4 incorporates more contemporary satellite altimetry data than EGM2008.

Huang, J. and Veronneau, M. (2015) evaluated the fit of EGM2008 and EIGEN-6C4 to (231079) land gravity data and (2668) leveling derived height anomalies in Canada. Here the root mean squared difference of EGM2008 compared to the gravity data was $0.82 \mathrm{mGal}$ smaller than EIGEN-6C4 which indicates a better fit, although the value does not exceed the $1.8 \mathrm{mGal}$ estimated accuracy of the gravity data. In contrast, the standard deviation of the difference between EIGEN-6C4 and the leveling derived height anomalies is shown to be around $1 \mathrm{~cm}$ smaller than that for EGM2008.

This improved result is attributed to the satellite gravity data having the only influence on the derived EIGEN-6C4 model coefficients for lower degrees. In contrast for EGM2008, more of the spectrum is influenced by the terrestrial gravity data, which causes it to fit terrestrial gravity data better than EIGEN-6C4. The better fit of EIGEN6C4 to the leveling derived height anomalies (which is an independent data set) indicates the satellite gravity data provides a better signal than terrestrial for geoid determination for the longer wavelengths (Huang, J. and Veronneau, M., 2015).

The same result is paralleled in New Zealand. EGM2008 fits the terrestrial gravity anomaly data with approximately $0.5 \mathrm{mGal}$ smaller standard deviation than EIGEN-6C4, and EIGEN-6C4 fits the leveling derived heights anomalies better than EGM2008 with an approximately $0.5 \mathrm{~cm}$ smaller standard deviation. More interestingly, the EIGEN-6C4 model appears to have a marginally better agreement with the leveling derived height anomalies than NZGeoid2009 which had a $4.8 \mathrm{~cm}$ standard deviation (0.2 cm larger).

\subsection{Summary}

In this Chapter a description of global gravity models (GGM) and how they are used to approximate the disturbing potential gravity field/ geoid height anomalies and gravity disturbances has been given. The global gravity models consist of spherical harmonic coefficients and coefficient standard deviation estimates. The coefficients are used in equations 5.4 and 5.5 to determine the geoid height and gravity anomalies.

Thirty seven satellite only global gravity models and 9 combined global gravity models have been evaluated up to their maximum degree and order and compared against terrestrial gravity data and leveling derived height anomalies. 
Of the thirty seven satellite only models GO_CONS_GCF_2_DIR_R5 provided the best fit to the terrestrial gravity (with a RMS of $37.6073 \mathrm{mGal}$ and standard deviation of 22.1671 taken of the inner $68 \%$ of the distribution since it is leptokurtotic) and leveling derived height anomalies data sets(with a RMS of $29.59 \mathrm{~cm}$ and standard deviation of $29.60 \mathrm{~cm}$ again taken of the inner $68 \%$ of the distribution).

For the nine combined models EGM2008 fit the terrestrial gravity data best and EIGEN6C4 best fit the leveling derived height anomalies. This is the same as the result obtained by Huang, J. and Veronneau, M. (2015) in Canada. Huang, J. and Veronneau, M. (2015) attribute this result to computation of EIGEN-6C4 having not used the terrestrial gravity data at all to determine low order spherical harmonic coefficients, instead using the new highly accurate GO_CONS_GCF_2_DIR_R5 and GRACE satellite data. So it doesn't fit the terrestrial data as well as EGM2008 which used the terrestrial gravity data to determine a wider spectrum of coefficients. However the better fit of EIGEN-6C4 to the leveling data, which is an independent data set, suggests that the satellite data is slightly more reliable at longer wavelengths than the terrestrial for geoid determination. 


\section{Chapter 6}

\section{Airborne Gravity across New Zealand}

\subsection{Introduction}

This chapter introduces the practice of airborne gravimetry, which is the method of measuring gravity from an aircraft. A national airborne gravity survey has been conducted over New Zealand and the details of the survey are given. The necessary reductions needed to transform the collected airborne gravity data in to gravity anomalies are discussed in detail. An accuracy assessment of the data is given by considering the difference in collected data at flight line intersection points, the agreement of the data with terrestrial gravity measurements and the discrepancies amongst data collected along flight lines which have been flown multiple times. Overall the data have been assessed to be accurate to approximately $3 \mathrm{mGal}$.

The airborne data set offers a spatially uniform set of measurements made with consistent apparatus, which will be used to enhance the existing terrestrial gravity data particularly where these data are sparse and near-offshore regions.

\subsubsection{What is Airborne Gravimetry?}

Airborne gravimetry is a method of making vertical acceleration measurements with apparatus that are mounted on an aircraft. The history of airborne gravity dates back as far as the 1950's (LaCoste, 1967 Huang, 2012) but, as discussed in the introduction, it has become more prevalent and reliable with the advent of accurate kinematic global positioning systems developed in the late 1980's.

Contemporary examples of the use of airborne gravimetry include the surveys conducted in Switzerland in 1992 (Klingele et al, 1996) and Greenland in 1991-1992 (Brozena, 1991), Mongolia in 2004-2005 (Forsberg et al. 2007), Taiwan in 2007 (Hwang et al, 2007) and the South Korean survey in 2008-2009 (Bae et al 2012, Yang 2013 and Jekeli 2013).

Airborne gravimeters measure the total vertical acceleration and so the positioning measurements make it possible to accurately model and remove specific forces associated 
with aircraft motion to obtain a gravity signal e.g. they must be corrected for the vertical accelerations experienced by the aircraft derived from GNSS data, the effect of the measurements not being made along a vertical gravity gradient (the tilt correction) and the Eotvos effect which is the perceived increase or decrease in the force of gravity when traveling west or east respectively (Bell et al, 1991).

The resolvable wavelength of the airborne data is dependent on a number of factors. For example, the measurement logging frequency and flight speed (i.e. if the gravimeter only logs once a second and the measurement platform (aircraft) is moving at $50 \mathrm{~m}$ per second then the shortest spatial wavelength that can be resolved is $100 \mathrm{~m}$ ) and similarly the flight line spacing has a similar effect on the cross track direction. Also, the airborne signal is often masked in high frequency high amplitude noise which needs to be removed. To do this an along track filter is applied however the filter also acts on the underlying gravity signal and so affects the resolvable wavelength and accuracy of the airborne data. This is discussed in details by Childers (1999), and in sections 6.3.5 and 6.6.

Various types of gravimeter are used for airborne gravity, for example the Chekan-AM gravimeter which is based on a dual quartz flexible pendulum element system with fluid dampening (Forsberg et al, 2015) or upgraded LaCoste and Romberg model S gravimeters which are spring-based relative gravity meters, (e.g. used by Hwang et al, 2007 and Brozena, 1991) that were originally designed to be used on board a ship at sea. They are mounted on a stabilized platform, which ensures the gravimeter is pointing along the vertical gravity gradient (Olesen, 2002). This is the type of gravimeter which has been used in a national airborne survey across New Zealand and is discussed in detail in the following sections.

The key advantage of airborne gravity is that areas which would be inaccessible by land or sea (i.e. over ranges or shallow coastal areas) can be surveyed more easily when flying, and so this method was chosen to improve the uniformity and density of the existing New Zealand gravity network by conducting a nationwide survey. 


\subsection{The New Zealand national airborne gravity sur- vey}

The airborne survey was conducted in two campaigns, the first between September and October 2013 and the second between February and June 2014 .

During the survey periods two survey flights were performed per day but averaging only 3 survey days per week due to intermittent poor weather conditions. Ideally the flight conditions should be smooth to avoid unnecessary aircraft motion which leads to spurious gravimeter measurements.

The surveyed region covers New Zealand's North and South Islands and Stewart Island with around 150 flight lines which are typically spaced $10 \mathrm{~km}$ apart, theoretically this is the resolvable wavelength of the data set (Hwang, 2007).

At the $10 \mathrm{~km}$ spatial resolution it is possible to determine a $2-5 \mathrm{~cm}$ accurate quasigeoid from airborne gravity

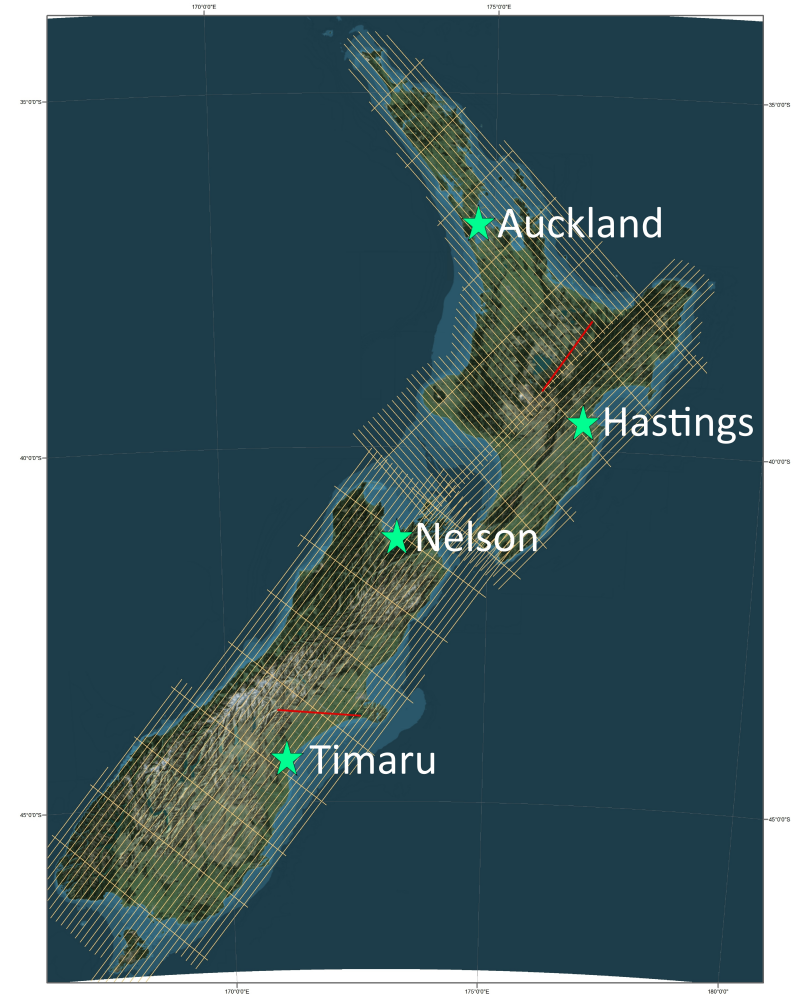

Figure 6.1: The surveyed flight lines (in white) and calibration lines (in red). The four hangers used during the survey are marked with stars. data (Bruton, 2000). The length of the lines ranged from around $100 \mathrm{~km}$ for the shorter lines on the North Island to around $600 \mathrm{~km}$ across the South Island. The longer lines in the South were typically flown in two $300 \mathrm{~km}$ length pieces to ensure the aircraft had enough fuel to return.

Twenty one of the flight lines are "cross lines" which intersect with a number of the other lines. These are spaced every $50 \mathrm{~km}$ and differences between data collected along these lines and those they intersect with were to check the internal consistency of the data (see section 6.6.1).

Each line has been flown at a near constant speed of 130 knots and measurements were made by the gravimeter at a rate of $1 \mathrm{~Hz}$ so that data points were acquired approximately every 65 metres.

Lines were flown as close as possible to the highest topography along them (at a near 
constant altitude), this was typically between 2000 (in the flatter regions of the North Island) and 4000 metres (in the rougher topography of the South Island).

There are also two "calibration" lines. The first is directly above High Level Road near Lake Taupo in the North Island, and the second runs along Thomson's Track in the South Island (marked in red on figure 6.1). These lines were flown over multiple times to assess the repeatability of the data which were collected.

Also terrestrial gravity observations were collected along the calibration lines at ground level. Measurements were made every $500 \mathrm{~m}$ and were used as ground truth comparison for the airborne data. This was done since the airborne data are dense due to the repeat flights so a robust comparison can be made.

Flights were based out of four separate locations, Hastings, Auckland, Nelson and Timaru, which were chosen so that a minimal amount of transiting was necessary between flight lines. The furthest distance to transit was around $100 \mathrm{~km}$ when flying from Timaru to fly the far west lines on the South Island.

At each base location an absolute gravity value has been obtained where the aircraft was parked using a Lacoste and Romberg model D relative gravimeter. These relative gravity values were tied into the existing terrestrial gravity network using a GNS Science owned software package (Gsolve) which corrects repeat relative terrestrial gravity observations for tidal effects and determines the gravity value at the observation points (compensated for gravimeter drift) by least squares. The data were tied into the existing gravity network (at BM2217 and ACQT for the North Island and B470 and AFQL on the South Island) with a standard deviation of approximately $0.1 \mathrm{mGal}$. 


\subsubsection{The Aircraft}

The aircraft which was used for the survey was a Piper Chieftain (ZK-RDT). It is a twin engined aircraft which was chosen for its long endurance (of around $2000 \mathrm{~km}$ ) due to the typical 6 hour flight length required to ensure optimal data capture. It can normally seat 8 passengers and has a maximum operating weight of over $3000 \mathrm{~kg}$ which meant it was suitable to house all of the equipment necessary for the gravity survey.

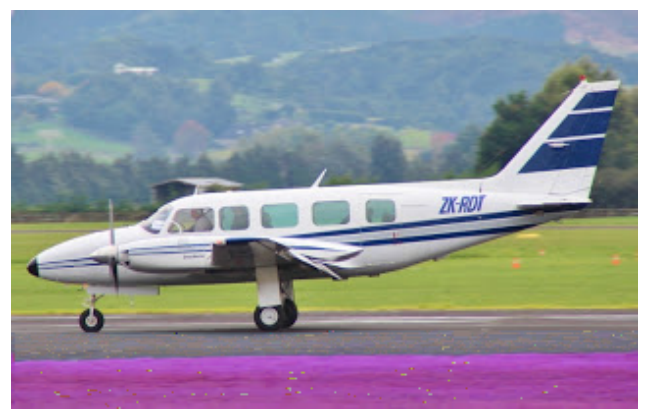

Figure 6.2: Piper Chieftain ZK-RDT

The aircraft was fitted with an autopilot which was used during the flight to maintain a steady air speed and consistent elevation. The autopilot estimated the aircrafts elevation using barometric measurements, in turbulent conditions the aircraft was susceptible to elevation changes. The pilot offset these elevation changes by adjusting the elevation of the aircraft's height relative to values determined by GNSS measurements.

\subsubsection{The GNSS and post processing methods}

The aircraft was fitted with a Novatel DL-4 L1 L2 GNSS receiver (serial number NYB06110012) and a permanent antenna (AT2774-41NTW-TNCF-000-RG-26-NM) mounted on the roof 1.32 metres (52") from the cabin floor where the gravimeter is placed (this can be seen in figure 6.3).

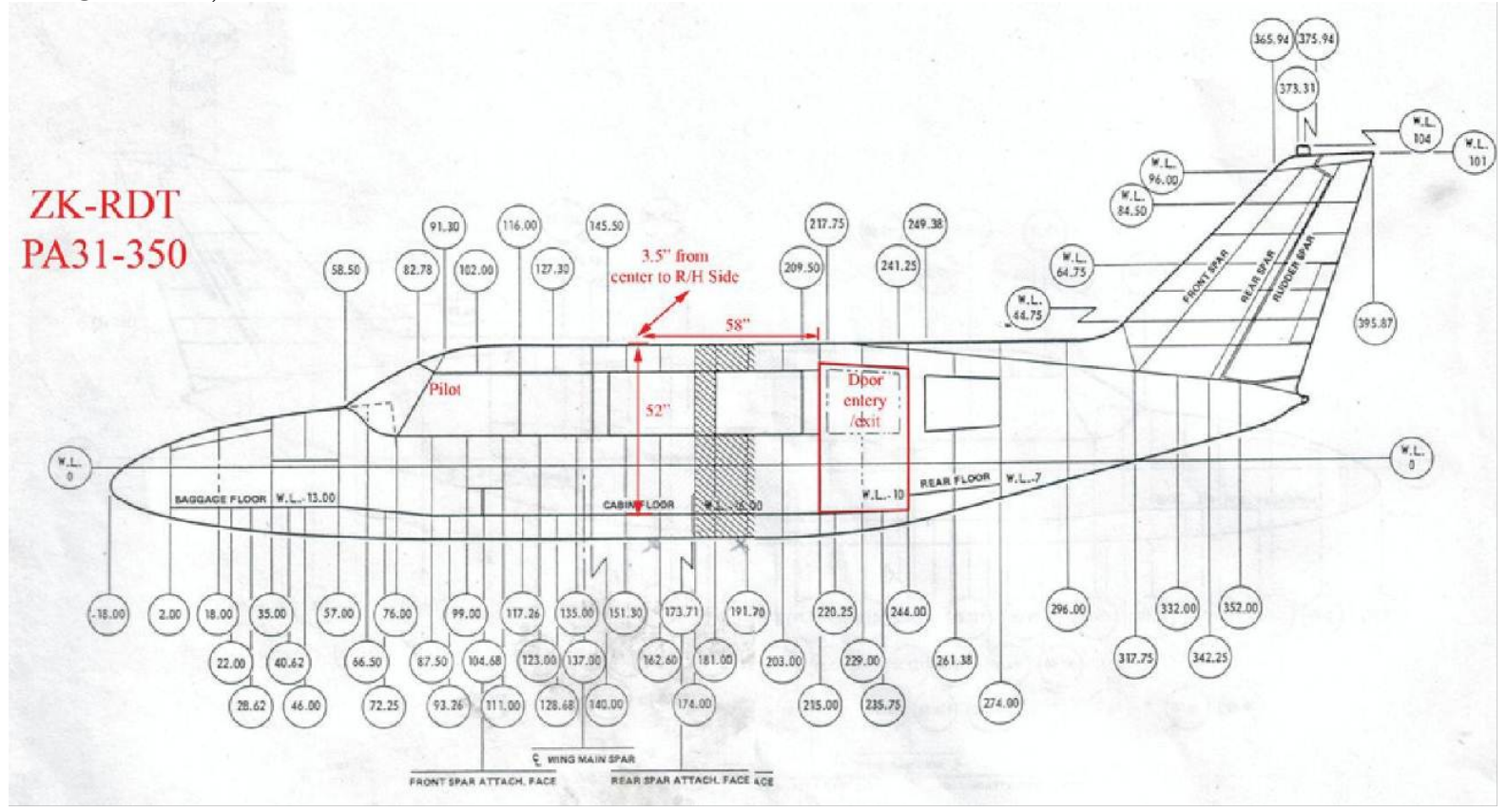

Figure 6.3: Piper Chieftain ZK-RDT schematic diagram 
The GNSS receiver logged at a rate of $2 \mathrm{~Hz}$ and output 2 data file types,

- a NMEA file, which provides the receiver's position and additional information about the determined position (e.g. how many satellites were used)

- and a PDC file which provided the information needed to calculate a position (e.g. satellite range, orbits and clock models of the satellites)

The GNSS data files were post-processed by Land Information New Zealand using the software package Waypoint GrafNav 8.40 (GrafNav/GrafNet User Guide, 2013). The software only required the PDC to calculate the position of the receiver.

The software package Waypoint GrafNav 8.40 was chosen since it performed optimally over other software during a pilot airborne survey the year previous to the national survey and additionally produces accuracy estimates of positioning calculations, which may prove useful for further study. It was specifically developed to handle airborne kinematic GNSS data, and so it was used to obtain a DGNSS (Differential Global Navigation Satellite System) solution for each flight.

\section{Processing Method}

From the GPS PDC data collected a position solution was determined by the baseline DGPS method. This method required supplementary GPS data from base stations, in order to determine the baseline processing. All base station data were provided by the Land Information New Zealand LINZ PositionNZ network (http://apps.linz.govt.nz/positionz/, last accessed 2016). The network consists of 33 continuously operating reference stations across mainland New Zealand and the Chatham Islands, and the data at the stations are recorded every 30 seconds.

Up to 6 base stations were used to process the solutions and were selected by assessing the extent of each flight line in QGIS and picking those which ensure approximately $100 \mathrm{~km}$ coverage of the flight line.

The "GNSS Airborne (High Altitude)" method was used and the option "Dual frequency carrier phase" was selected since it proved to give better accuracy on baselines longer than $10 \mathrm{~km}$.

The data were then assessed for gaps after processing, typically based on poor satellite visibility/ the geometry of the satellite positions. The gaps were resolved by lowering the elevation masks (which filter out signals from satellites below a specified angle of elevation above the horizon) from the default $12^{\circ}$ until the gap was resolved, typically around $9^{\circ}$. 


\subsubsection{The gravimeter}

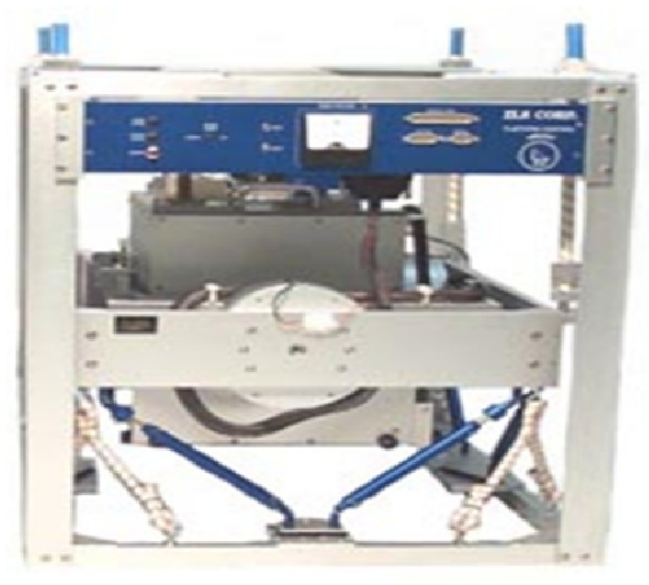

Figure 6.4: Upgraded ZLS LaCoste and Romberg S80 gravimeter used for the New Zealand national airborne gravity survey.

for by the platform.
An upgraded ZLS LaCoste and Romberg S80 meter was used for the airborne data collection (LaCoste, 1967). It is a zero-length spring based relative gravity meter which sits on a stabilized, damped platform.

The platform aligns the gravimeter with the vertical gravity gradient (over a period of around 240 seconds) using 2 accelerometers $\left(f_{x}\right.$ and $\left.f_{y}\right)$ and gyroscopes and the dampening reduces any high frequency vibrations. This means the meter is ideally suited to being mounted on an aircraft to determine gravity variations across a survey area, where fine scale roll and pitch manoeuvres of the aircraft along a flight line are adjusted

The gravimeter largely consists of a hinged beam supported by a zero-length spring. When the force of gravity changes the beam begins to move. The spring length (tension) can be adjusted to null the beam and the change in spring tension can be used to measure the change in gravity. Internally large scale accelerations of the beam are reduced using an air dampener on the model of gravimeter used for the data collection. The stabilized platform is damped by oil shock absorbers and bungee cords around the base. This is depicted in figures 6.5 and 6.4 .

When the gravimeter is in motion it is not practical to constantly null the beam so the gravimeter must be read whilst the beam is in motion. Mathematical analysis of the gravimeter dynamics (described in Dehlinger (1978) pg 110 to 113 and the Model S instruction manual) shows that a relative gravity measurement, made at time $t$, can be calculated from the gravimeter component values: spring tension $(S)$, beam position $(B)$ and cross coupling $(C)$, as follows,

$$
g_{t}=\alpha\left(S_{t}+k \dot{B}_{t}+C_{t}\right) .
$$

The constant values $\alpha=0.9899$ and $k=30$ have been provided by the manufacturer, where $k$ is called the beam scale factor and $\alpha$ converts the counter units of the spring tension, beam derivative and cross coupling to $\mathrm{mGal}$.

$\dot{B}_{t}$ is the differential of the beam position $B$ with respect to time and has been calculated from the raw data by a central difference,

$$
\dot{B}_{t}=\frac{B_{t+1}-B_{t-1}}{2} .
$$




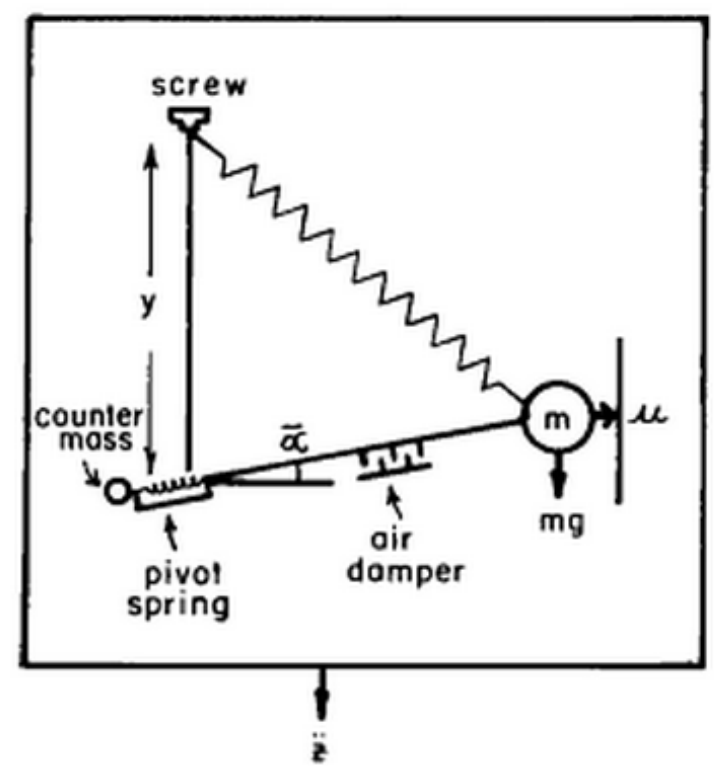

Figure 6.5: Schematic design of the Lacoste and Romber model S gravimeter adapted from Dehlinger (1978).

The cross coupling is computed by the gravimeter automatically and is given by,

$$
C=c_{1} \dot{B}^{2}+c_{2}\left(f_{y} B\right)+c_{3}\left(f_{x} \dot{B}\right)+c_{4}\left(f_{y} \dot{B}\right)+c_{5}\left(f_{x}^{2} \dot{B}\right)
$$

where the constants $c_{1}, c_{2} \cdot c_{3}, c_{4}$ and $c_{5}$ have been provided by the manufacturer.

The meter logs its data directly to a laptop and saves a new file once an hour and the individual measurements are logged once every second.

The gravimeter has certain limitations which require consideration when it is used in a practical environment. For example, the system consists of highly sensitive moving parts and so when it is subjected to extreme/jerky motions it can easily be broken.

To avoid any unnecessary breakages when conducting the national survey, during takeoff, landing and turning from one flight line to the next, the spring tension motor was turned off and the beam was clamped down, and also the platform torque motors (which are used to align it with the vertical) were switched off.

Once the whole system was re-engaged on a flight line it took approximately 5 minutes for the gravimeter to begin logging meaningful gravity data again. However, the transient time for the gravimeter was a known factor during the survey design and was accounted for by extending the flight lines by approximately $10 \mathrm{~km}$; also for lines that were flown in two pieces, a sufficient overlap was ensured. 


\subsection{Gravity reductions}

This section documents the initial processing step taken to reduce the raw airborne gravity measurements to free air gravity anomalies, namely

- the components of the gravimeter output file, spring tension $S$, beam position $B$, cross coupling $C$, cross accelerometer $f_{x}$ and long accelerometer $f_{y}$ and

- processed DGNSS data which contains ellipsoidal (GRS80) latitudes, longitudes and heights, $(\phi, \lambda, h)$.

The following 7 key steps were taken (Olesen, 2002).

1. Calculate relative measurements from the raw components of the gravimeter for all readings by equation 6.1 .

2. Obtain the following constants: A "still" relative gravity measurement, $g_{B}$ (using the Lacoste and Romberg S metre described above), and the absolute gravity value $G_{B}$, where the "still" data was recorded. These measurements were made where the aircraft was stored over night as described in section 6.2.

3. From the GPS data, calculate the vertical accelerations, $\ddot{h}_{t}$, and use these to synchronise the relative gravity measurements, $g_{t}$ with GPS data in the time domain.

4. Using the gravimeter accelerometer data, $f_{x}$ and $f_{y}$ and cross/along track GPS derived accelerations, $a_{x}$ and $a_{y}$, calculate the tilt correction, $\Delta g_{\text {tilt }}$.

5. From the GPS data, calculate the Eotvos effect, $\Delta g_{\text {eot }}$, ellipsoidal absolute gravity effect $\gamma$, and free air effect $\delta g_{F A}$ for each record in the GPS data file.

6. Calculate the airborne free air anomaly using the following equation,

$$
\Delta g_{F A}=g_{t}-g_{B}+G_{B}-\ddot{h}+\Delta g_{t i l t}-\Delta g_{e o t}-\gamma+\delta g_{F A} .
$$

7. Low pass filter the along track free air anomalies to remove high frequency noise.

By expanding on the points above, this section details the initial processing steps taken for the airborne gravity measurement to obtain free air anomalies along each flight line.

A worked example is followed throughout this section, using a particular flight line in the airborne data set, to demonstrate the typical amplitude of the initial relative gravity data and the required reductions necessary to obtain a meaningful signal for further processing.

\section{Relative gravity measurements from raw components of the gravimeter}

For each flight line the individual gravimeter output components were extracted and the relative gravity value has been computed for each measurement point along by equation 6.1 .

Figure 6.6 shows the relative gravity readings along a particular flight line. The amplitude of the signal is of the order $10^{4} \mathrm{mGal}$, which is significantly larger than the \pm 200 $\mathrm{mGal}$ that is expected of the gravity signal. 


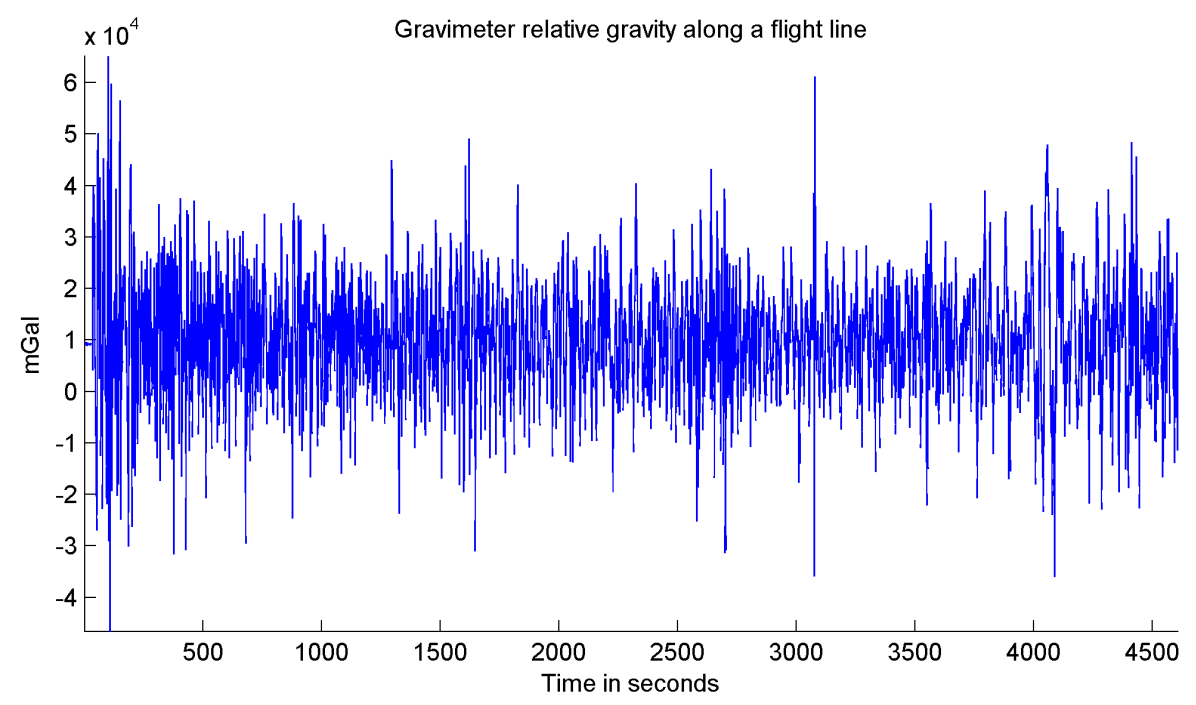

Figure 6.6: The relative gravity from the gravimeter computed from the raw gravimeter components. The amplitude of the signal is $10^{4} \mathrm{mGal}$, this is significantly larger than what is expected from the gravity field. Corrections must be made to obtain a meaningful gravity signal.

\section{Obtaining absolute gravity values from the relative measurements}

Relative gravity measurements $g_{B}$ and absolute gravity values $G_{B}$ have been obtained at each of the base locations. The relative measurements made by airborne gravimeter are prone to a small amount of drifting, i.e. the base value of the gravimeter given by $G_{B}-g_{B}$ steadily increases over time. The drift rate has been estimated at around 0.3 $\mathrm{mGal}$ per month from a long term series of measurements made at the same location where the gravimeter is stored when it is not in use.

To minimize the effect any drift in the relative measurements during the survey $g_{B}$ was recorded on a daily basis. The daily readings have then only been used to calculate absolute gravity (vertical accelerations) from the relative measurements made on that day of surveying.

The absolute gravity along each flight line has been obtained by the following equation,

$$
G_{t}=g_{t}-g_{B}+G_{B}
$$

$G_{t}$ is shown in figure 6.7 . 


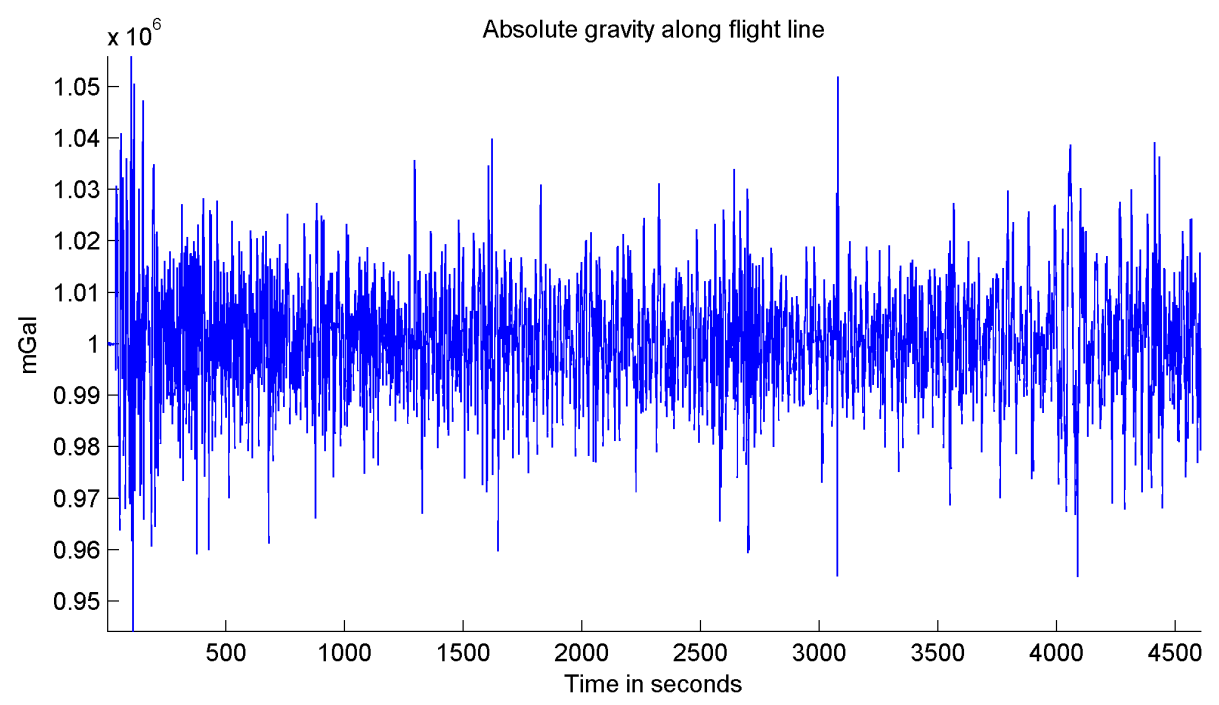

Figure 6.7: The derived absolute vertical accelerations along the flight line measured by the gravimeter.

\subsubsection{Vertical Accelerations and Synchronising the GPS with the Gravimeter measurements}

The gravimeter is measuring the total acceleration along which its axis is aligned. When the gravimeter is mounted on a moving body (such as a boat or an aircraft) $G_{t}$ additionally contains contributions from non gravitational accelerations. To recover the gravity signal the effect of the platform motion must be modeled and subtracted.

Both the gravimeter and the GPS contain a time channel. To accurately locate the gravimeter measurements and correct for the effects of the platform motion the two signals must be synchronised.

When the gravimeter is mounted on an aircraft, the vertical accelerations of the aircraft's motion are significantly greater and higher frequency than any other factor contributing to the measured acceleration $G_{t}$. For the most part, the gravimeter is just measuring the vertical accelerations of the aircraft's motion. This is useful since the large similarity between two signals can be used to synchronise the data sets.

The vertical accelerations, $a_{z}=\ddot{h}$, of the aircraft motion can be deduced by taking two successive central differences to the elevation above the reference ellipsoid $h$ given by the GPS. This is,

$$
\ddot{h}_{t}=\frac{h_{t+2}-2 h_{t}+h_{t-2}}{4},
$$

where $h$ is given in units of $0.001 \mathrm{~cm}$. Figure 6.8 shows the GPS vertical accelerations for the example flight line. 


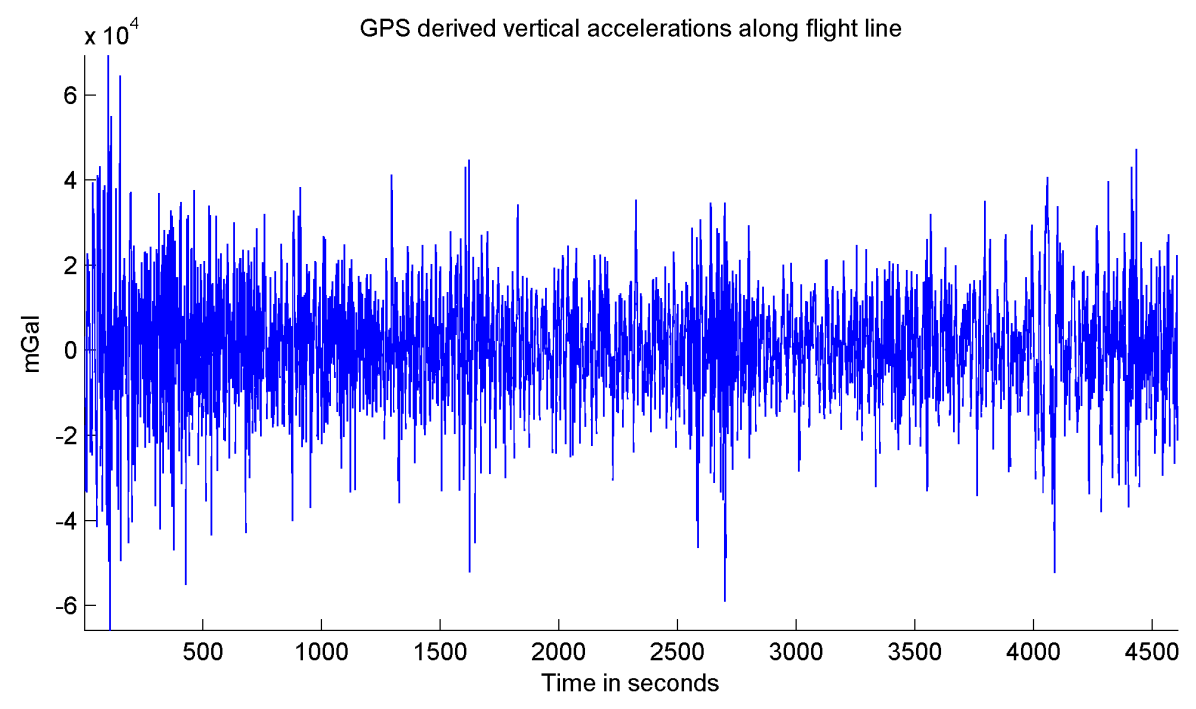

Figure 6.8: The GPS derived vertical accelerations along the example flight line. Similar to the relative gravimeter data the amplitude of the GPS derived vertical accelerations is around $10^{4}$ indicating that the aircraft's motion is the likely source of high amplitude signal in the gravimeter measurements. For this reason the GPS derived vertical accelerations must be subtracted from the gravimeter signal.

$G_{t}$ and $\ddot{h}$ can then be used to find any offset between the gravimeter and GPS time channels by finding the argument which maximizes a convolution of them. i.e. for $G(t+\delta t)=G_{t+\delta t}$ and $\ddot{h}_{t}=h \ddot{h}(t) \delta t$ is given by,

$$
\delta t=\underset{t}{\operatorname{argmax}} \int_{-\infty}^{\infty} G(t-\tau) \ddot{h}(t) d \tau .
$$

The convolution is then just a multiplication in the Fourier domain giving,

$$
\hat{Q}=\hat{G} * \hat{\ddot{h}}
$$

For $Q_{t}$ the inverse Fourier transform of $\hat{Q}, \delta t$ is given by $\delta t=\operatorname{argmax}_{t}(Q)$. At the start of the survey the gravimeter time was set using a GPS clock. The GPS sends a pulse exactly every second to the gravimeter which is used to set the time precisely on the second. For this reason, for the data collected during this survey, $\delta t$ is an integer number of seconds.

For the example flight line data the offset was determined to be 16 seconds. The following figure shows the GPS derived vertical accelerations (in red) and de-meaned (just for illustrative purposes) gravimeter data for a small section of track. The agreement between the two signals is striking in terms of the amplitude and general correlation. 


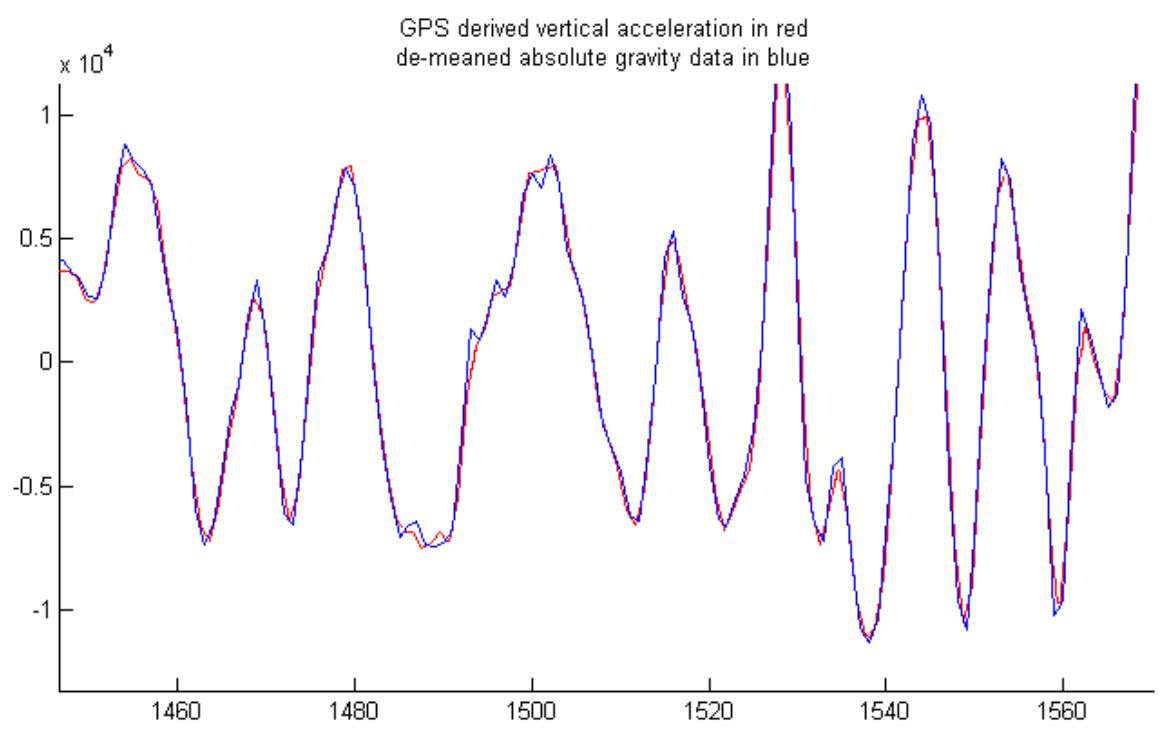

Figure 6.9: A zoomed in plot of the GPS derived vertical accelerations (red) and demeaned gravimeter absolute gravity along the example flight line (blue). The two signals are remarkably similar indicating most of what the gravimeter is measuring is the vertical accelerations of the plane.

\subsubsection{Tilt Correction}

The gravimeter has two accelerometers (one pointing across the meter, $f_{x}$, and one along the meter, $f_{y}$ ) which are used in conjunction with gyros and torque motors to level the platform. However the platform leveling is not immediate and so the gravimeter axis can be perturbed from the vertical gravity gradient. When this happens the gravimeter is less sensitive in the vertical direction and sensitive to horizontal accelerations which causes biases and correlated errors in the signal. Horizontal accelerations are commonplace during the survey where there has been a cross wind along the flight line and during turbulence, and so a tilt correction is important, particularly for these flight conditions.

The tilt correction is given by,

$$
\Delta g_{t i l t}=g-\sqrt{g^{2}+f_{x}^{2}+f_{y}^{2}-a_{x}^{2}-a_{y}^{2}}
$$

where $\mathrm{g}$ is the nominal value of gravity, and $a_{x}$ and $a_{y}$ are the across and along track accelerations (all given in mGal) ( Peters and Brozena, 1995).The general amplitude of this correction can be seen in figure 6.10 of the tilt correction along the example flight line.

The readings from the accelerometers are included in the gravimeter output file but are given in millivolts. The millivolt readings can be transformed into mGal using a scale factor which must be calculated. This calculation was performed for the gravimeter used for the airborne survey and the details of this process are given in appendix 1 . 


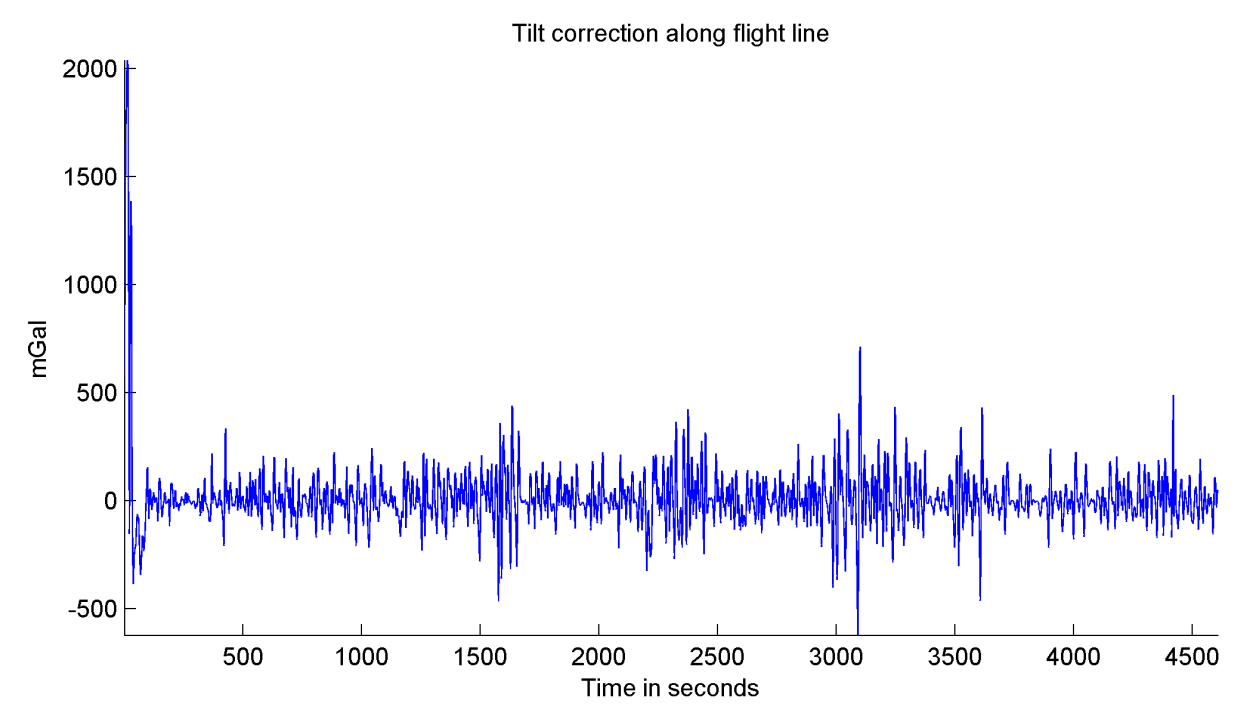

Figure 6.10: The calculated tilt correction along the example flight line.

\subsubsection{The Eotvos effect}

The Eotvos effect is a change in the strength of gravity due to the aircraft's velocity relative to the rotation of the earth (Bell, 1991). When moving in the same direction as the rotation of the earth (i.e. eastbound) the angular velocity is greater so there is a perceived decrease in the force of gravity, and conversely when moving west. The following steps outline how to calculate this effect following Harlan (1968).

First calculate the radii of curvature of the prime and equatorial meridians at each point $\left(\phi_{t}, \lambda_{t}\right)$ as follows,

- $N_{t}=\frac{a}{\sqrt{1-e^{2} \sin \left(\phi_{t}\right)^{2}}}$ Radius of Curvature for equatorial meridian

- $M_{t}=\frac{a\left(1-e^{2}\right)}{\left(1-e^{2} \sin \left(\phi_{t}\right)^{2}\right)^{3 / 2}}$ Radius of Curvature for Prime meridian

where $a=6378137$ metres is the equatorial radius of the GRS80 reference ellipsoid and $e^{2}$ is the eccentricity squared of the GRS80 reference ellipsoid as given in section 2.2.1. Next, calculate the Longitudinal and Latitudinal derivatives in radians per second. This can be done numerically by taking a central difference and multiplying by $\pi / 180$. This gives the following,

- $\dot{\phi}_{t}=\frac{\phi_{t+1}-\phi_{t-1}}{2} \pi / 180$

- $\dot{\lambda}_{t}=\frac{\lambda_{t+1}-\lambda_{t-1}}{2} \pi / 180$

Then the velocity in the East and North directions on the surface of the ellipsoid are given as,

- $V_{E}(t)=N_{t} \cos \left(\phi_{t}\right) \dot{\lambda_{t}}$

- $V_{N}(t)=M_{t} \dot{\phi}_{t}$ 
The Eotvos effect is then given by,

$$
\begin{gathered}
\Delta g_{E o t}=-\frac{V_{N}^{2}(t)}{a}\left[1+\frac{h_{t}}{a}+f\left(2-3 \sin \left(\phi_{t}\right)^{2}\right)\right]-\frac{V_{E}^{2}(t)}{a}\left[1+\frac{h_{t}}{a}-\right. \\
\left.\left.f \sin \left(\phi_{t}\right)^{2}\right)\right]-2 V_{E}(t) \omega \cos \left(\phi_{t}\right)\left(1+\frac{h_{t}}{a}\right) \times 10^{5} \text { for mGal }
\end{gathered}
$$

here $f$ is the ellipsoid flattening as given in section 2.2.1 and $h_{t}$ is the height above the reference ellipsoid in metres.

The following figure shows the Eotvos correction computed for the example flight line. The correction is positive since the flight line was flown from west to East.

Eotvos correction along flight line

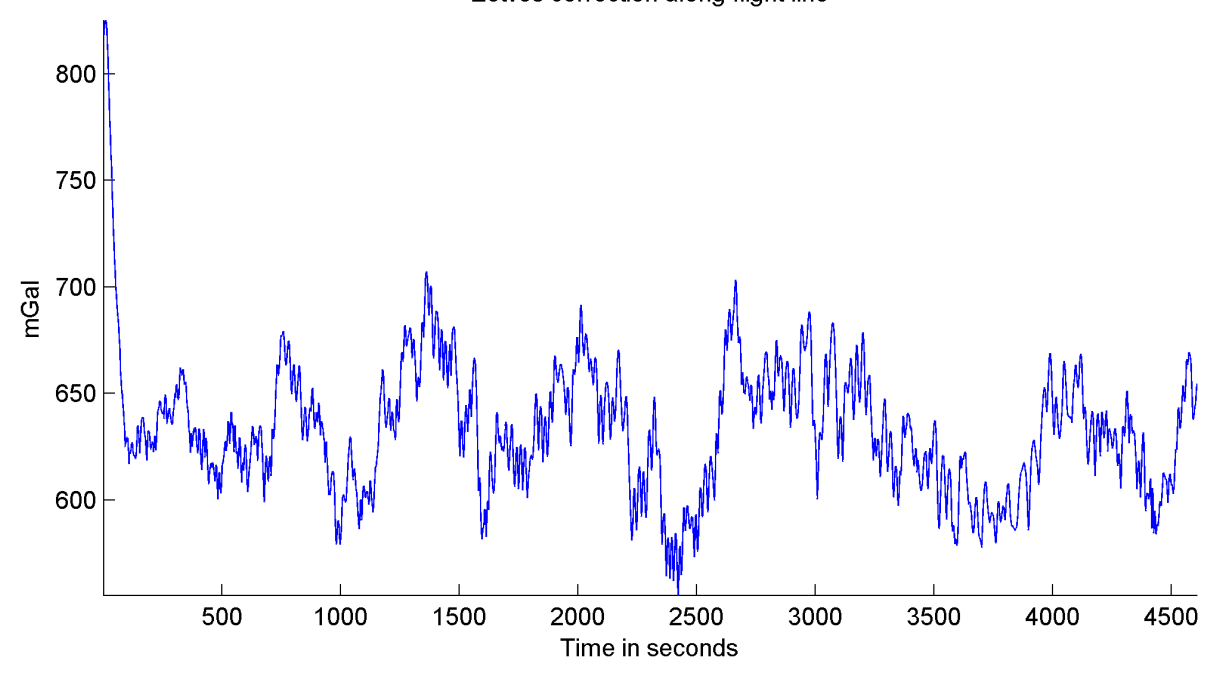

Figure 6.11: This figure shows the Eotvos correction along the example flight line. The correction is positive since the flight line was flown from South-West to North-East.

\subsubsection{The Free Air gravity anomaly}

As discussed in section 2.2.2 the free air anomaly is calculated by subtracting the gravity field of a reference ellipsoid $\gamma$, and the free air effect, $-\delta g_{F A}$ from the gravity observations. The GRS80 coordinates acquired by the airborne GPS have been used to obtain the normal gravity $\gamma$, and the GRS80 height minus the Eigen-6C4 geoid undulations (to estimate the normal-orthometric height) has been used to calculate $-\delta g_{F A}$.

The free air anomaly is then given a long each flight line by,

$$
\Delta g_{F A}(t)=G(t)-\ddot{H}(t)+\Delta g_{t i l t}(t)-\Delta g_{E o t}(t)-\gamma(t)+\delta g_{F A}(t)
$$

Figures 6.12 and 6.13 show the two corrections $-\gamma$ and $\delta g_{F A}$ along the example flight line and figure 6.14 shows the free air gravity signal $\Delta g_{F A}(t)$. 


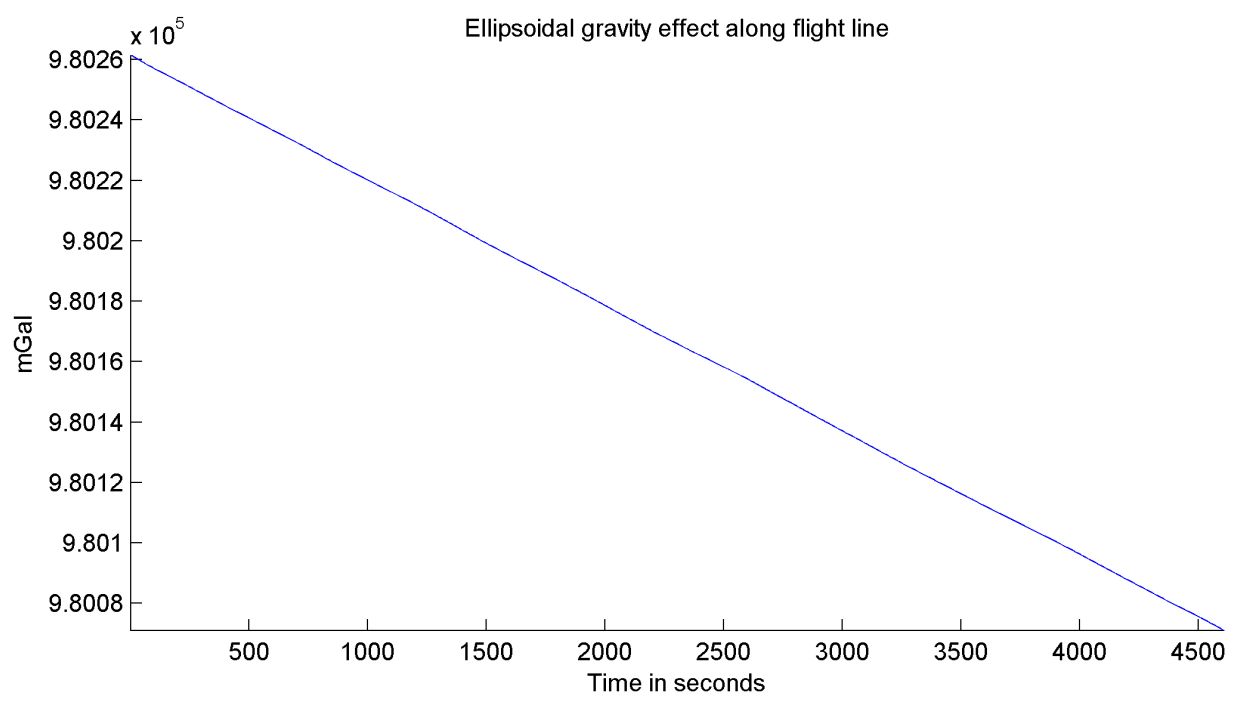

Figure 6.12: The ellipsoidal gravity along the example flight line. There is a negative gradient in the correction since ellipsoidal gravity is strongest at the poles and the flight line was flown from south-west to north-east.

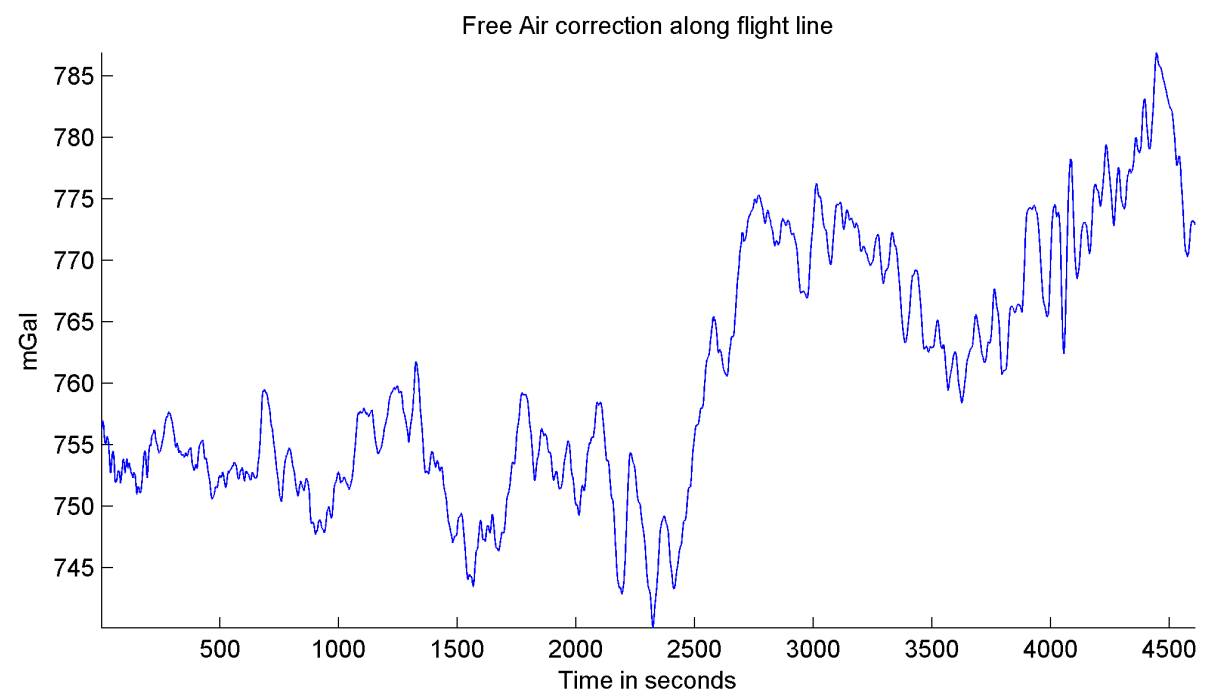

Figure 6.13: The free air correction along the example flight line. 


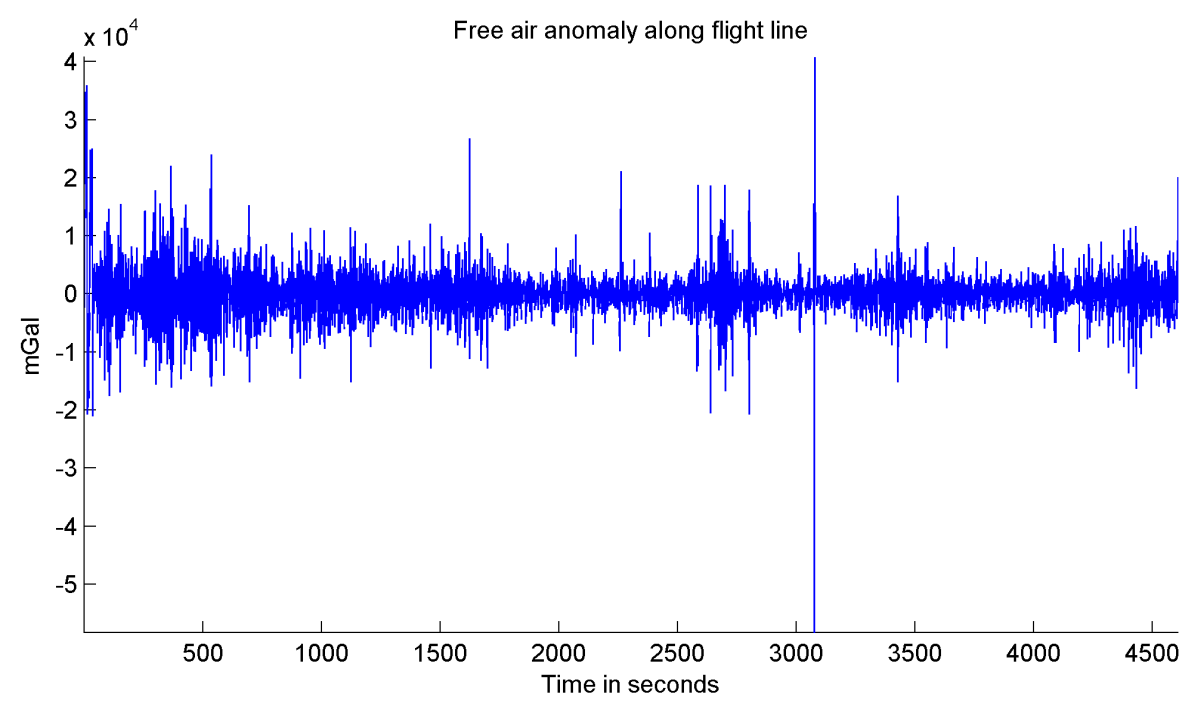

Figure 6.14: The noisy free air gravity signal along the example flight line. Note that the signal amplitude is significantly higher than the expected $\pm 200 \mathrm{mGal}$ range of the free air gravity anomaly. The signal is completely masked in high amplitude, high frequency noise.

\subsubsection{Low Pass Filtering}

The free air anomaly for each individual flight line is masked in high frequency, high amplitude noise. This can be seen in figure 6.14 of the final free air gravity profile (after applying the discussed corrections to the gravimeter data) along the example flight line. The amplitude of the signal is around $10^{4} \mathrm{mGal}$ however the anticipated gravity signal is more likely to be within the range of around $\pm 150 \mathrm{mGal}$ as can be seen in figure 4.3 of the terrestrial free air anomaly.

The standard approach to remove the noise is to implement a low pass filter along the flight line data in the time domain (Olesen, 2002). A Gaussian low pass filter has been used for all flight line data in the survey. The filter has the form,

$$
g(t)=\frac{1}{\sqrt{2 \pi} \sigma} e^{-\frac{t^{2}}{2 \sigma^{2}}}
$$

where the frequency response is given by the Fourier transform, $\hat{g}(f)=e^{-f^{2} 2 \sigma_{f}^{2}}$ and with $\sigma \sigma_{f}=\frac{1}{2 \pi}$. The filtered free air anomaly, $\Delta \tilde{g}_{F A}(t)$, is given by

$$
\Delta \tilde{g_{F A}}(t)=\int_{-\infty}^{\infty} \Delta g_{F A}(t-\tau) g(t) d \tau
$$

The filter attenuates the high frequencies in the signal so that the noise is removed. Figure 6.15 shows the noisy free air anomaly data after a Gaussian filter with a $\sigma$ parameter of 120 seconds is used (for illustrative purposes, this parameter is optimized in section 6.6), in red the EGM2008 global gravity anomaly along the line is also shown to demonstrate the effectiveness of the filter. 


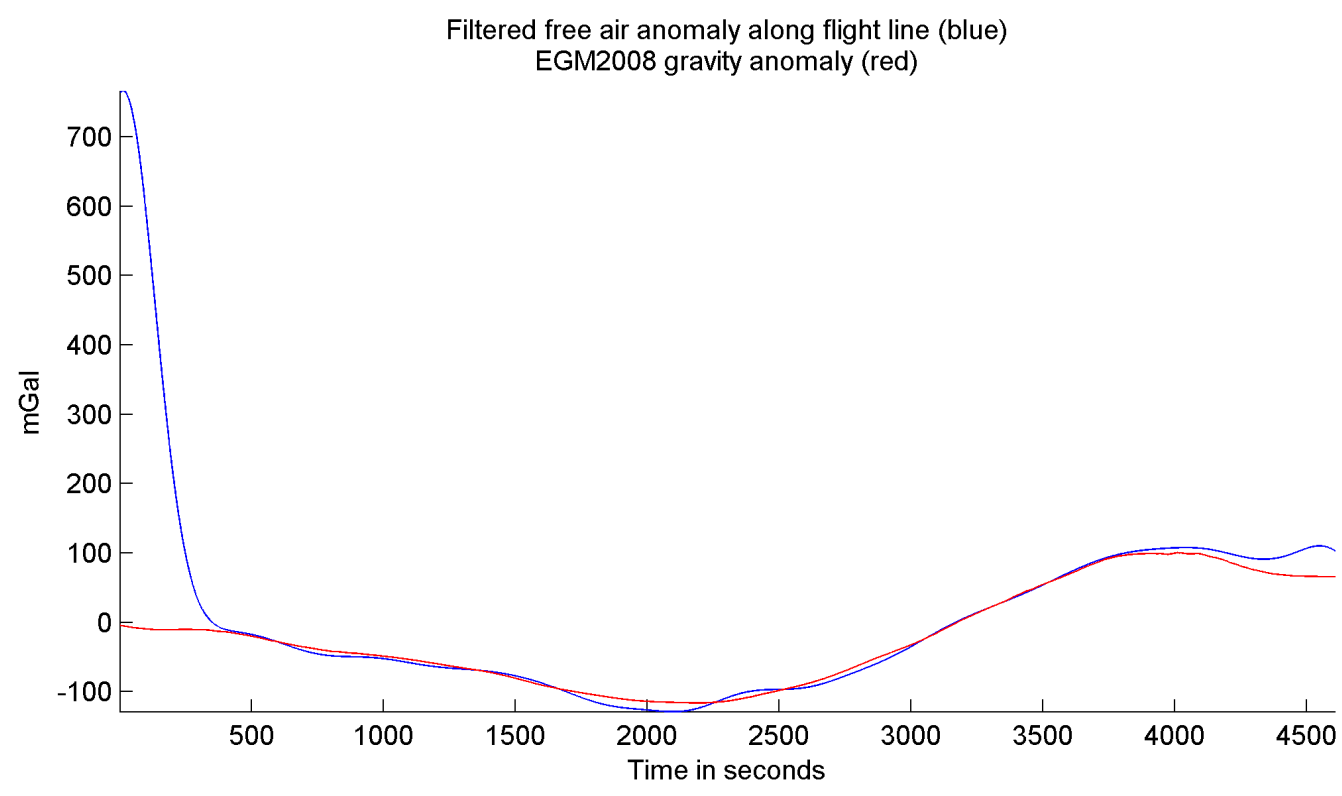

Figure 6.15: This figure shows the filtered free air gravity signal (in blue) along the example flight line and the EGM2008 gravity anomaly along the same line (red) for comparison.

\subsubsection{Terrain corrections for the airborne gravity data}

The operation to correct for the vertical component of gravity caused by the terrain with terrestrial gravity measurements is split into two parts. First the effect of a slab of topography the height of the gravity observation is removed, then the effect of mass missing below the slab and additional mass above the slab is corrected for with the terrain correction as discussed above. The vertical gravity component of the deviations of the terrain from this slab beyond some maximum radius (taken before as $120 \mathrm{~km}$ ) is considered negligible.

The airborne measurements are made at least $500 \mathrm{~m}$ above the topography. If the same two step operation is performed for the airborne gravity data the thickness of the Bouguer slab would be substantially larger and would necessitate a significantly larger maximum radius for the terrain corrections to adequately remove the effect of the topographic deviations from the slab. Instead an amended form of the equation 2.13 given in section 2.2.3 gives the vertical gravitational effect of an annulus of topography of height $h$ for a measurement made at height $e$ above the geoid as,

$$
\delta g(r, R, h, e, \rho)=0.0419 \rho\left(\sqrt{R^{2}+(e-h)^{2}}-\sqrt{r^{2}+(e-h)^{2}}+\sqrt{r^{2}+e^{2}}-\sqrt{R^{2}+e^{2}}\right) .
$$


Where $\delta$ is the resolution of the DEM, as before calculate a factor $f$ which corresponds to the ratio between the area of the piece of terrain and the area of the annulus,

$$
f=\frac{\delta^{2}}{\pi\left(R^{2}-r^{2}\right)}
$$

and the terrain correction $\delta g_{T C}$ for the piece of terrain $x_{p}, y_{p}, h_{p}$ is given by,

$$
\delta g_{T C}=f \delta g(r, R, h, e, \rho) .
$$

This way, the vertical gravitational effect of a piece of topography right down to the geoid can be calculated directly. The geometry of this correction is illustrated in figure 6.16.

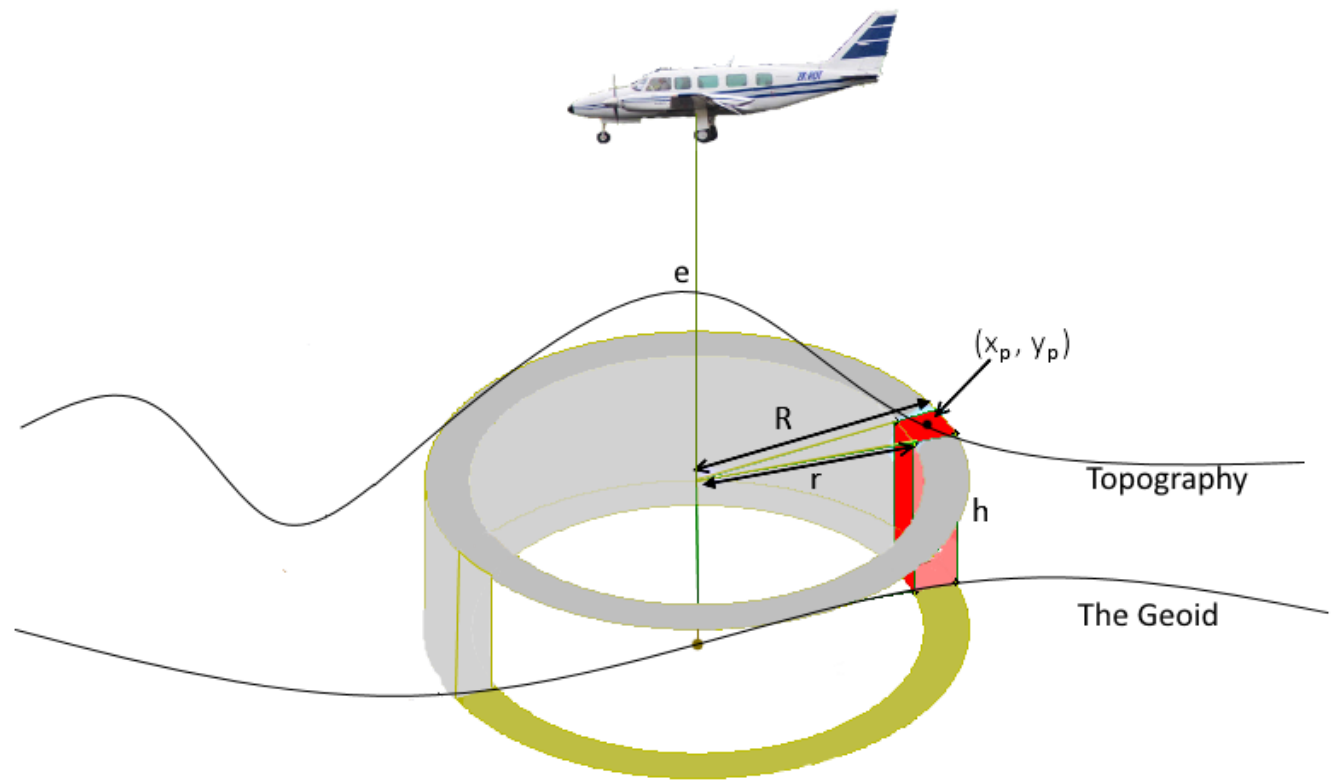

Figure 6.16: An illustration of the geometry for a segment of Hammer's terrain correction for an annulus of topography $e-h$ below the observation location.

Terrain corrections have been computed for each of the airborne gravity observations. The near zone effect of topography on the airborne gravity observations is significantly reduced due to the elevation of the gravity observations above the earth's surface. Moreover, in total there were close to 1000000 measurements made. For these reasons it was considered advantageous to use the $64 \mathrm{~m}$ resolution digital elevation model over the $8 \mathrm{~m}$ resolution model since it reduced the computation time from around 45 days to about 5 without any substantial loss in accuracy.

When computing the along track Bouguer anomalies, the terrain correction is added to the noisy free air gravity anomaly before the line is filtered to reduce topographic aliasing. 


\subsection{Detecting erroneous sections of track}

The free air airborne gravity signal for each flight line (after applying a Gaussian filter with $\sigma=120 \mathrm{~s}$ ) was assessed for erroneous sections of track by comparing it to neighbouring flight line data and a gravity anomaly derived from the EGM2008 coefficients. Any biases/dc offsets in the flight line data were not considered during this process, only the general shape of the gravity profile.

There appeared to be three predominant causes of erroneous data.

1. At the start and end of each flight line, the along track filtering process has edge effects where the full filter kernel is not used.

2. At the start of each flight line, there is a transient period of up to 5 minutes where the gravimeter settles down to logging realistic acceleration data following the beam being un-clamped and torque motors being reengaged.

The beam was clamped and the platform torque motors were turned off during times when the aircraft turned. The beam was clamped because the accelerations during aircraft turnings exceeded the range of the gravimeter and could potentially damage it. The stabilized platform has a maximum tilt angle smaller than needed for the aircraft to manoeuver quickly between lines, necessitating the torque motors to be turned off.

However, the transient time for the gravimeter was a known factor during the survey design and was accounted for by extending the flight lines by approximately $10 \mathrm{~km}$; also for lines that were flown in two pieces a sufficient overlap was ensured. This meant that clipping the start of each flight line results in minimal data loss over the whole survey region. Moreover the change in the measured gravity due to heading, latitude and elevation coming onto a new flight line was approximated and the spring tension was offset prior to reengaging the torque motors and un-clamping the beam so that transient period was minimized.

3. The beam position has a maximum range of $\pm 9000 \mathrm{mV}$. However in extreme turbulence the vertical acceleration of the aircraft cause the beam to reach this threshold.

When the beam position changes the gravimeter adjusts the spring tension to pull the beam back to the null position. However when the beam position reaches the threshold the adjustments made by the gravimeter to the spring tension are excessive. It takes several minutes before the gravimeter is again logging realistic vertical acceleration data.

To flag the bad data without having to delete observations from the whole data base, a vector of weights for each flight line has been established. The weights have been assigned a one where the data are considered good and a zero for data in the bad sections of track. 
Detecting and flagging erroneous data at the start of the flight lines: The gravimeter measurements during the transient period at the beginning of each flight line approach the true vertical accelerations like a damped harmonic oscillator and the edge effect of the filter causes large deviations. Zero weights have been given during the start and end of each flight line to flag the data for removal/down weighting when they are further processed.
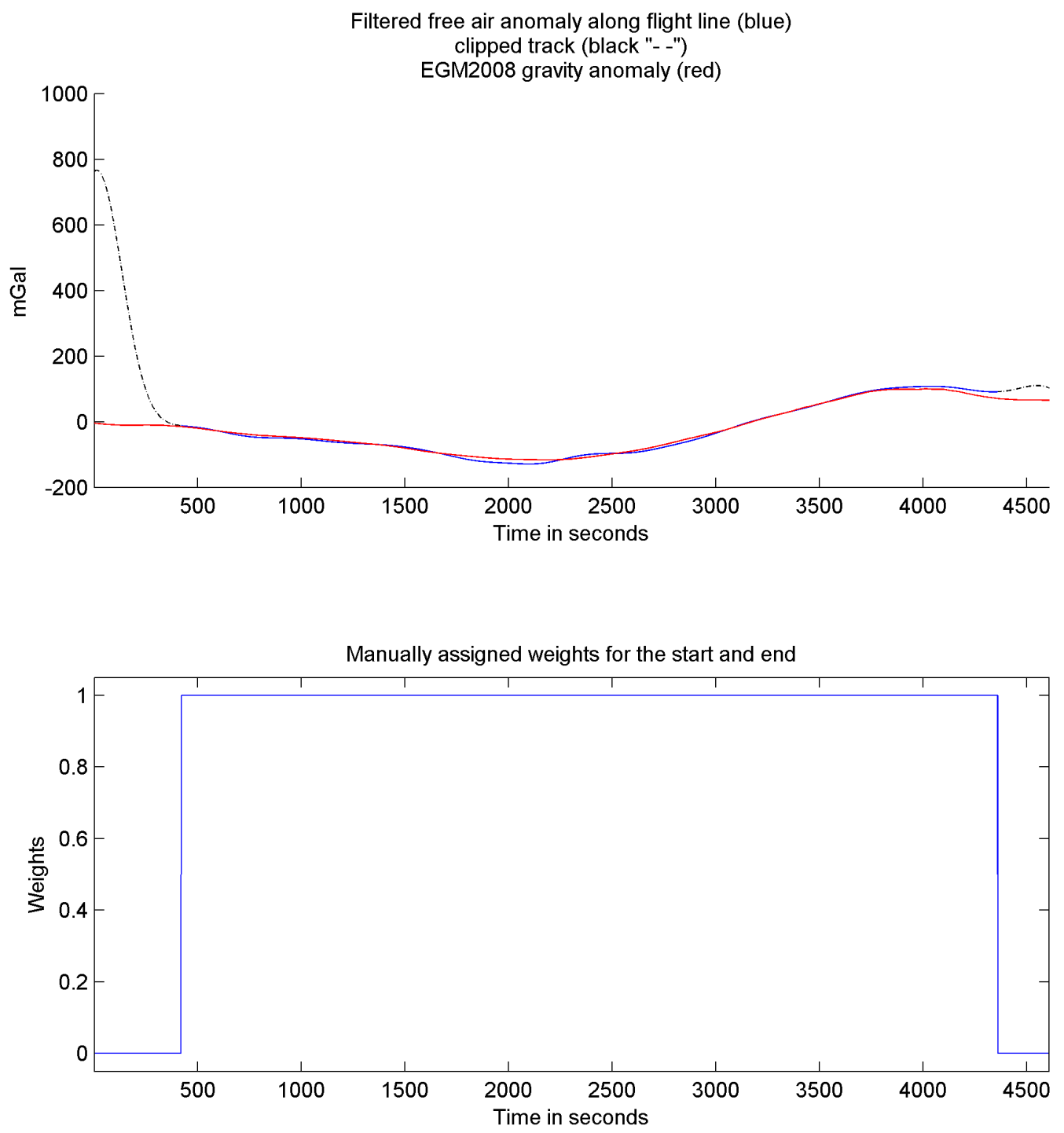

Figure 6.17: This figure demonstrates where zero weights were manually assigned to the erroneous sections of track at the beginning and end of the example flight line. The top plot shows the filtered signal (black), clipped filtered signal (blue) and gravity anomaly from EGM2008 (red) and the bottom plot shows the weights which have been manually assigned to the along track data. 
Detecting and flagging erroneous data along the flight lines Erroneous sections of data appear as brief patches of impulse noise in the raw unfiltered gravity signal and as a sudden high amplitude oscillation in the filtered signal as shown in the following figure.

These incidents were isolated by comparing the filtered airborne gravity signal to neighbouring flight line data using a grid and also comparing the gravity anomaly profile to EGM2008.
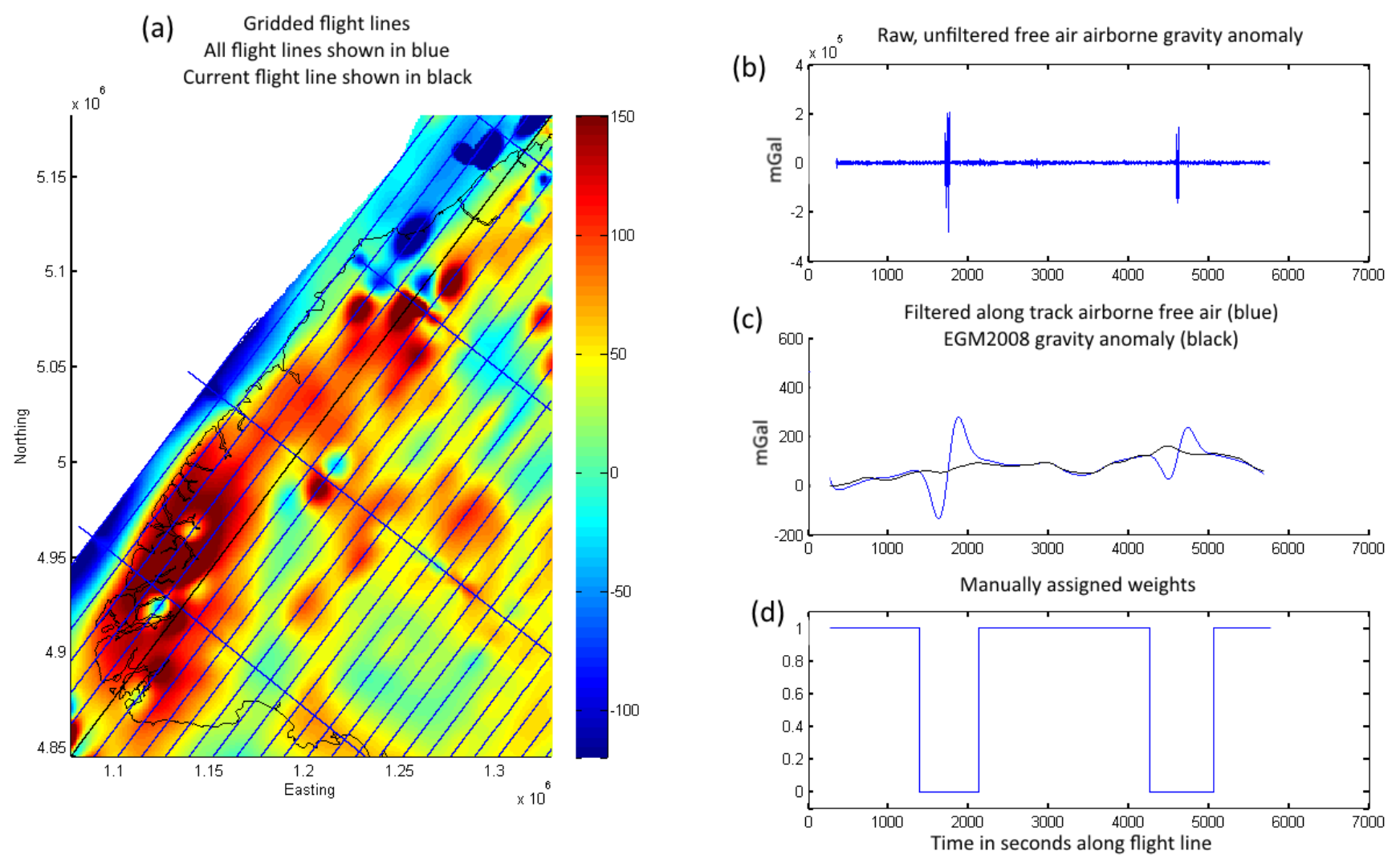

Figure 6.18: This figure illustrates where zero weights were manually assigned to the data. The gridded data are shown in subplot (a) with the flight line in black. These plots were used to examine the cross track agreement of the data. Subplot (b) shows the raw unfiltered free air gravity signal. Here there is some impulse noise characteristic of the beam reaching the threshold, around $1800 \mathrm{~s}$ and $4700 \mathrm{~s}$. Subplot (c) shows the filter signal (blue) and gravity anomaly from EGM2008 and subplot (d) shows the weights which have been assigned to the data.

The worst of these events have been visually identified and flagged in the along track data using the filtered free air anomaly signal and comparing it to neighbouring flight lines and a gravity anomaly at flight elevation derived from EGM2008. The total amount of data that have been identified as erroneous is $6.38 \%$. Figures $6.19 \mathrm{a}$ and $6.19 \mathrm{~b}$ demonstrates where data has been assigned a zero weight and has not been plotted. 


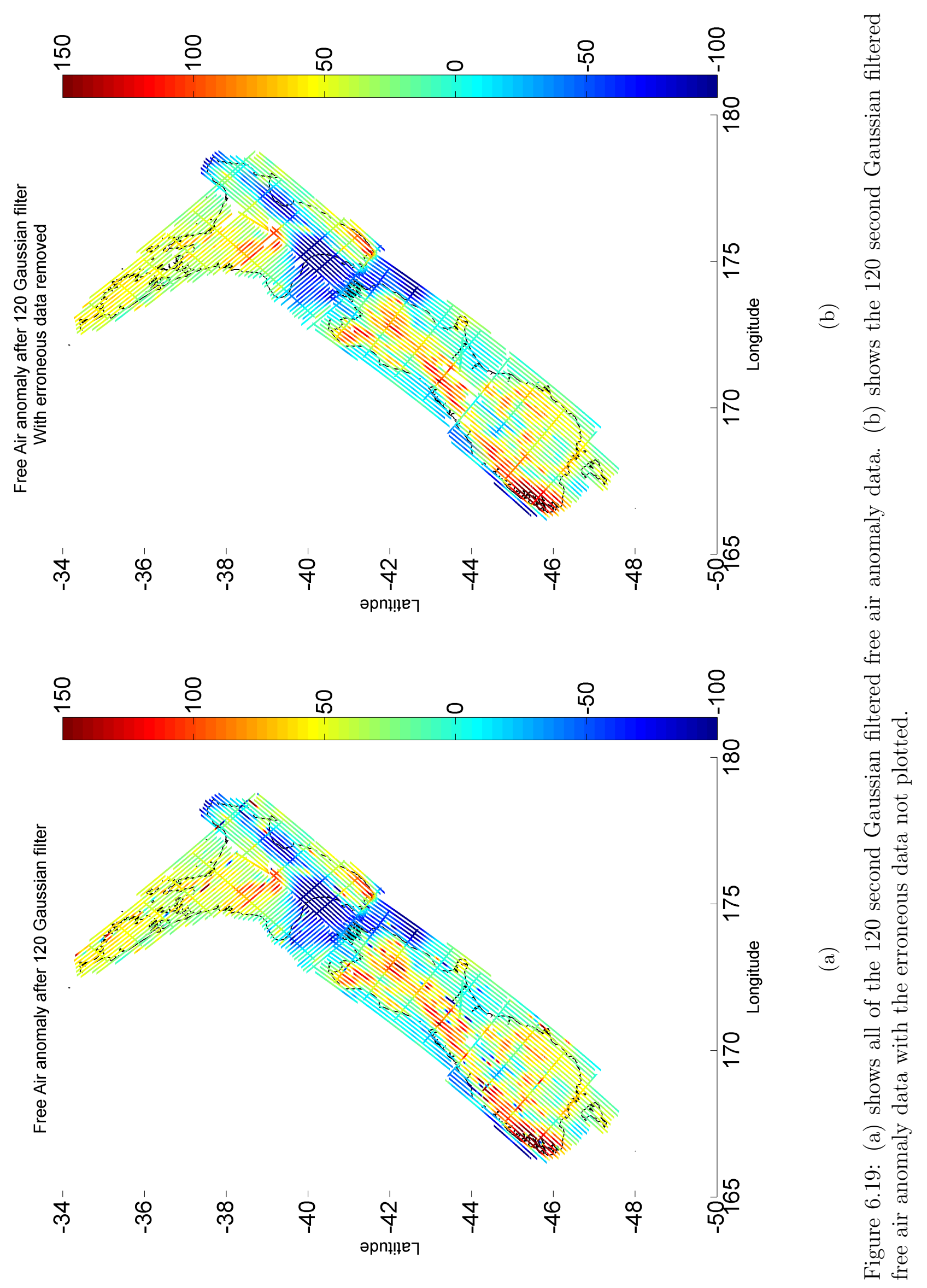




\subsection{Flight line biases}

Data along several flight lines appear to be offset from a gravity anomaly derived from the EGM2008 coefficients and the surrounding flight line data. Potential sources of the biases include the tilt correction (if the accelerometer millivolts to mGal scaling coefficients are modelled poorly), insufficiently accurate gravimeter still readings, GPS height errors and gravimeter operational errors which result in there being a difference between the spring tension counter on the gravimeter and what is recorded on the computer.

The following figure shows the free air anomaly along an example flight line and the gravity anomaly derived from the EGM2008 coefficients along the line.

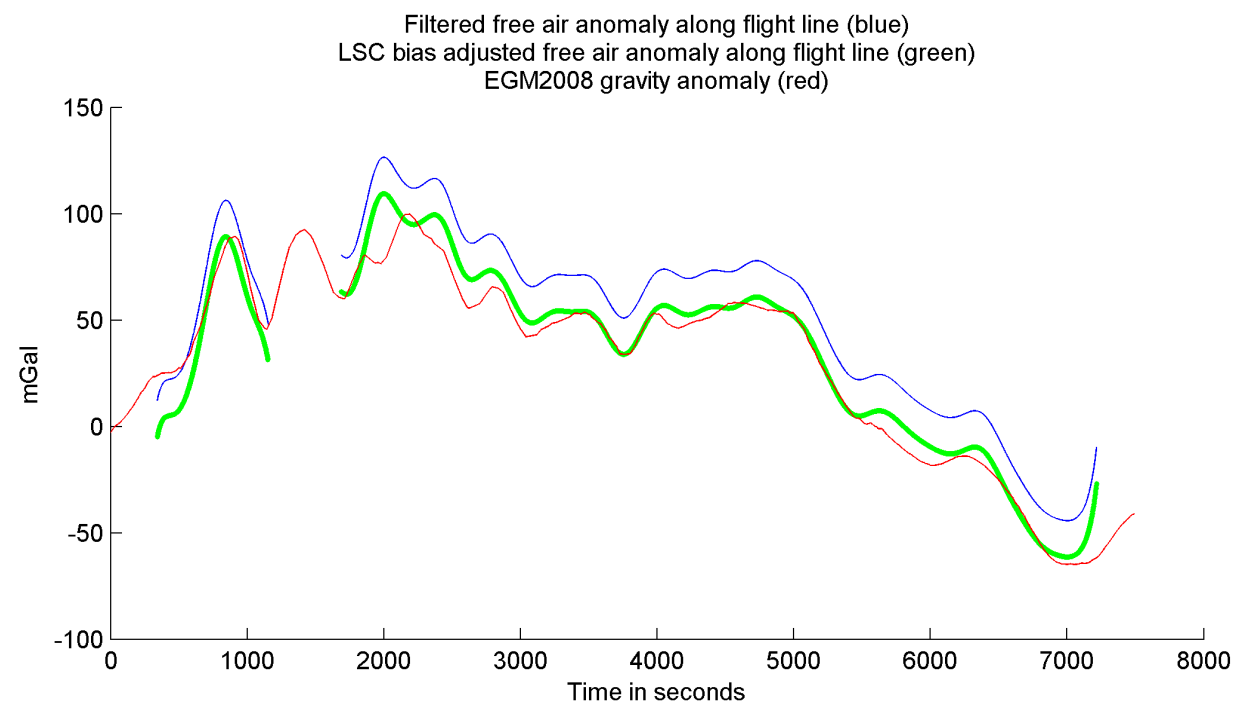

Figure 6.20: Along track free air profile showing a bias present in the airborne gravity data along flight line 23. Airborne data is plotted in blue, EGM2008 is plotted in red and the bias adjusted line by the methodology described in the following section is plotted in green. There is a clear dc offset between the red and blue lines, estimated by a mean difference to be $15.7 \mathrm{mGal}$. This bias is not present visually between the red and green lines. 
A bias, $b_{i}$ for each flight line $i$ has been estimated, using the methodology laid out in Reilly (1979), by the following procedure. Details regarding each of these steps are given in the remainder of this section.

1. First compute the Bouguer anomaly for each flight line in the airborne gravity data set.

2. Then calculate the EGM2008 gravity anomaly along each flight line at flight level.

3. Next remove the approximate effect of terrain in the EGM2008 gravity anomaly model.

4. Subtract the terrain corrected EGM2008 signal from the airborne Bouguer anomaly data to obtain residual gravity observations here denoted $\Delta g$.

5. Calculate the empirical covariance of the residual gravity observations $\Delta g$, and find the best fitting analytic logarithmic covariance model parameters.

6. Calculate the covariance matrix, $C_{g g}$, of residual gravity observations, the bias coefficient matrix $A$, and the diagonal regularization matrix $N$.

7. Calculate the biases $b$ by the following equation,

$$
b=\left(A^{T}\left(C_{g g}+N\right)^{-1} A\right)^{-1} A^{T}\left(C_{g g}+N\right)^{-1} \Delta g .
$$

$b$ is a 1 by $M$ vector, where $\mathrm{M}$ is the number of separate lines of data in the survey, i.e each entry in the vector $b$ is the bias for a flight line, which must be subtracted from the line data so that it becomes "bias free". Equation 6.15 consists of the matrix $C_{g, g}$, which is a covariance matrix that describes the spatially correlated signal in the residual gravity data $\Delta g, N$ which is a covariance matrix which describes the noise in the residual gravity observations and $A$ which describes which bias element each residual gravity observation will contribute to in the solution.

The inclusion of matrices $C_{g, g}$ and $N$ ensures the spatially correlated residual gravity signal and signal noise modeled by $N$ do not contribute to the determined biases. The method described above to compute flight line biases is advantageous over just estimating a mean offset from EGM2008 for each flight line since it takes into consideration the correlated signal between separate flight lines.

A terrain corrected EGM2008 is subtracted from the Bouguer gravity observations in step 4 to ensure the estimated biases, calculated for the residual signal $\Delta g$, are independent of the long wavelengths in the gravity field. EGM2008 was chosen for this procedure 
since it gave the best fit to the gravity data in section 5.1. The reason for using the Bouguer gravity anomaly and a terrain corrected EGM2008 gravity anomaly is to avoid topographical aliasing, Jekeli (2013) indicates terrain can introduce a bias of up to 8 $\mathrm{mGal}$ if not properly taken into account.

Advantages of using the bias adjustment procedure described above over the traditional cross-over adjustment method. Below the standard cross-over adjustment procedure for line bias estimation is described. It has been used historically for shipborne data for example the ship track data discussed in the Amos (2005), and similarly for airborne data e.g. the 1998 Greenland survey described in Forsberg et al (1999), in Tahiti by Shih et al (2015). The traditional method considers the difference between values recorded along different flight lines at intersection points and solves a least squares formulation of the flight lines bias based on the intersection differences.

i.e. Let $f_{j}, j=1,2,3, \ldots J$ and $f_{k}, k=1,2,3, \ldots K$ be two distinct sets of flight lines which run perpendicular to each other. Let $\mathbf{x}_{j, k}$ be the intersection point of lines $f_{j}$ and $f_{k}$ with $\Delta g_{j, k}$ the true gravity anomaly at that point.

If $\tilde{\Delta} g_{j, k}$ and $\tilde{\Delta} \psi_{j, k}$ are the measured gravity anomaly along lines $f_{j}$ and $f_{k}$ respectively at the point $\mathbf{x}_{j, k}$,

$$
\tilde{\Delta} g_{j, k}=\Delta g_{j, k}+\mu_{j, k}
$$

and

$$
\tilde{\Delta} \psi_{j, k}=\Delta g_{j, k}+v_{j, k}
$$

then the cross-over difference, $C_{j, k}$, is given by,

$$
\tilde{\Delta} g_{j, k}-\tilde{\Delta} \psi_{j, k}=\mu_{j, k}-v_{j, k}=C_{j, k} .
$$

Let $B_{j}=b_{1}, b_{2}, \ldots, b_{J}$ and $\hat{B}_{k}=\hat{b}_{1}, \hat{b}_{2}, \ldots \hat{b}_{K}$ be two distinct sets of biases for the two sets of perpendicular flight lines. Then for each intersection point,

$$
b_{j}-\hat{b}_{k}=C_{j, k}+\epsilon_{j, k}
$$

The biases $B_{j}$ and $\hat{B}_{k}$ can then be solved for by least squares, i.e. minimizing the set of $\epsilon_{j, k}$ 's. Hwang et al (2006) and Hwang et al (2007) use a weighted least squares solution.

The standard deviation of the set of all cross-over differences is typically used to assess the noise level of the data, this is described in detail in section 6.6. The cross-over adjustment results in a set of cross-over differences which have a standard deviation that is smaller than it was initially, e.g. in the 1998 Greenland airborne survey (Forsberg, 1999) the cross-over adjustment reduced the RMS of the crossover discrepancies by 1 mGal, and for Hwang (2007) it reduced the cross-over RMS of the difference by around 2 $\mathrm{mGal}$. However the reduction in the RMS error value does not necessarily mean the noise level in the new data is smaller, just that noise at the intersection points has been partly 
absorbed into the estimated biases, this degrades the quality of the noise assessment.

Moreover the biases will be in part based on the measurement errors at the intersection points. This is acknowledged by Olesen et al (2002) and Forsberg et al (2001) which both indicates that the traditional cross-over adjustment procedure (equation 6.19) will distribute the errors at measurement points into the estimated flight line biases. This problem is compounded by the fact the cross-over adjustment problem is typically poorly constrained (i.e. the flight line bias for each line is determined from very few points). Some of the flight lines in the national airborne survey only have one intersection point as can be seen in figure 6.29. Which means the total error in the airborne data at that intersection point will be absorbed by the flight line bias which is inappropriate.

Finally, section 6.6 illustrates that the along track filter applied to the airborne data additionally introduces intersection differences between flight lines. It would not be appropriate to allow these intersection differences to have influence over the bias estimation. This is acknowledged by Murray (2001) who describes this bias adjustment procedure as ineffective for airborne gravimetry due to gravity signal attenuation caused by the filter.

The alternative method described previously which is based on the Reilly (1979) formula, is advantageous compared to the traditional approach with respect to the issues raised above. The alternative method is not poorly constrained and is instead based on all the data. This reduces the influence of zero mean Gaussian noise in the independent measurements on the biases since the mean of the noise is more likely to be zero due to the larger sample size (i.e. the variance of the mean noise level is equal to the variance divided by the square root of the sample size). The random measurement noise is further excluded from the bias estimation using the Reilly (1979) method due to the inclusion of the matrix $N$ which has a regularization effect on the determined biases with respect to the estimated noise level of the flight line data. Finally, there is still some validity to the cross-over error analysis (discussed in detail in section 6.6) since the cross-over discrepancies have not been inappropriately parameterized.

Step 1. the Bouguer anomaly airborne gravity data: Figure 6.21 shows the calculated Bouguer gravity anomaly signal after a 120 second Gaussian filter along each separate flight line. The figure corresponds to step 1. 


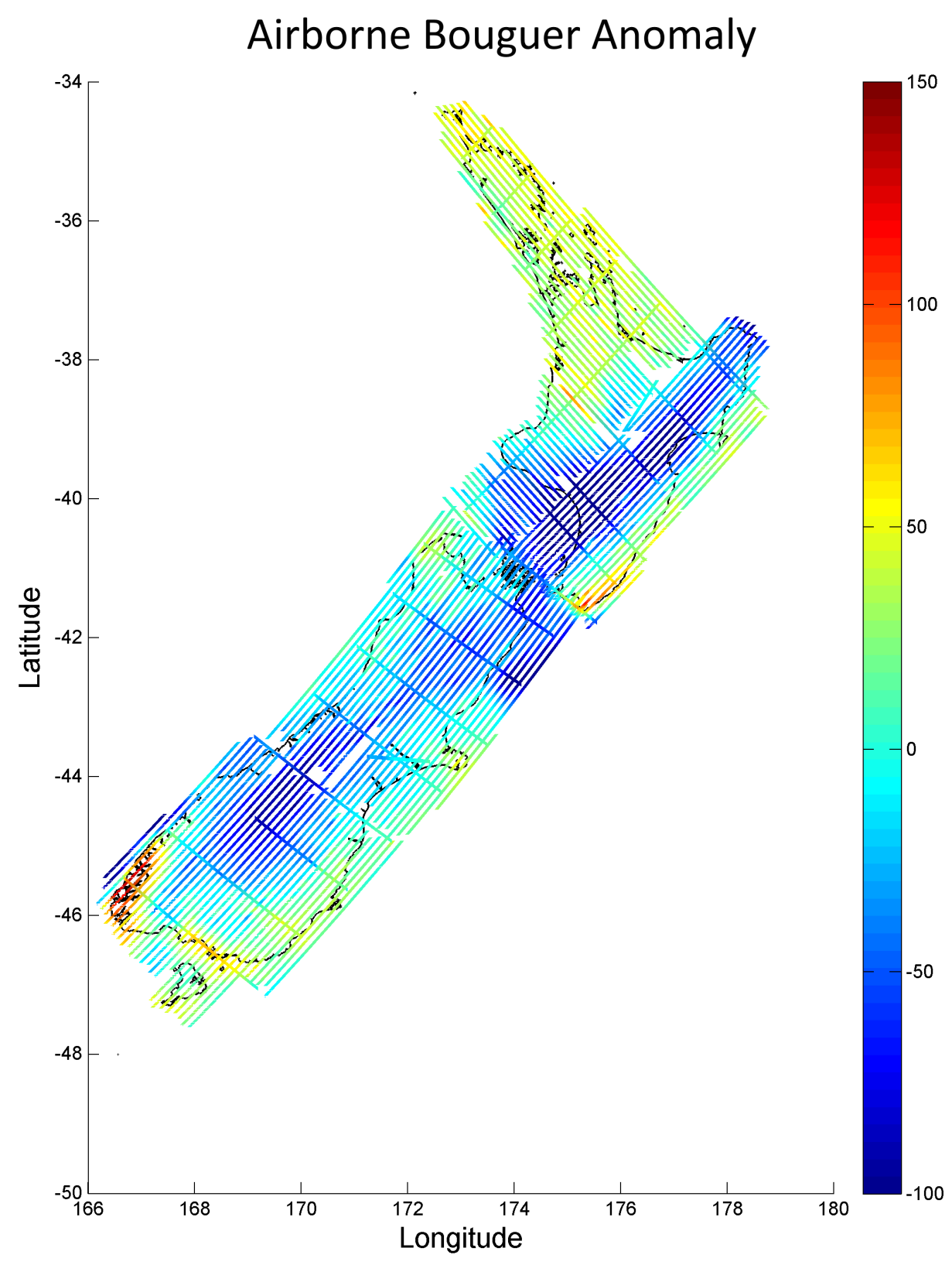

Figure 6.21: The airborne Bouguer anomaly data for each flight line. Only the data which have not been flagged as erroneous (white gaps) are shown here.

Steps 2,3 and 4. obtaining a zero mean random signal To ensure the estimated flight line biases $b$ are independent of the long wavelengths in the gravity field a long wavelength gravity anomaly from global gravity model EGM2008 was subtracted to obtain a residual gravity signal $g$. 
To avoid topographical aliasing when gridding residual gravity observations, $\Delta g_{1}$, by least squares collocation (see section 7.1) Forsberg and Kenyon (1995) says an airborne gravity observation $\Delta g$ can be broken into three parts,

$$
\Delta g=\Delta g_{1}+\Delta g_{2}+\Delta g_{3}
$$

where $\Delta g_{3}$ is a long wavelength gravity signal from some GGM (global gravity model) such as EGM2008, $\Delta g_{2}$ is the residual terrain effect and $\Delta g_{1}$ is the residual gravity signal.

The residual terrain effect $\Delta g_{2}$ is computed from a DEM which has had the long wavelength (approximately the same as the minimum wavelength in the GGM) topographic variations removed. This prevents the effect of the long wavelength topography present in the GGM being subtracted twice when obtaining $\Delta g_{1}$.

It is well known that the effect of terrain is distributive, i.e. if $F\left(t_{i}\right)$ is the effect of a piece of terrain denoted $t_{i}$, then for $t_{i}=\hat{t}_{i}+\bar{t}_{i}$ (i.e. the prism has been broken into two sections), $F\left(t_{i}\right)=F\left(\hat{t}_{i}+\bar{t}_{i}\right)=F\left(\hat{t}_{i}\right)+F\left(\bar{t}_{i}\right)$.

This property of the terrain effect is most commonly employed when using a Bouguer slab/shell plus the effect of topographic variations from the slab/shell i.e. the terrain has been broken into the average terrain (the Bouguer slab) plus high frequency terrain (variations from the slab).

For this reason, here the residual terrain effect, $\Delta g_{2}$ has been calculated in two parts as,

$$
\Delta g_{2}=\Delta \hat{g}_{2}-\Delta \bar{g}_{2}
$$

which is the effect of the full terrain minus the effect of the long wavelength terrain. Then

$$
\Delta g=\Delta g_{1}+\Delta \hat{g}_{2}-\Delta \bar{g}_{2}+\Delta g_{3}
$$

so that

$$
\Delta g_{1}=\left(\Delta g-\Delta \hat{g}_{2}\right)-\left(\Delta g_{3}-\Delta \bar{g}_{2}\right) \text {. }
$$

$\left(g-\Delta \hat{g}_{2}\right)$ is the observed Bouguer anomaly (as seen in the previous figure) and $\left(\Delta g_{3}-\Delta \bar{g}_{2}\right)$ is the GGM gravity anomaly approximately corrected for the effect of long wavelength topography. In the absence of the along track biases $\Delta g_{1}$ would be a zero mean random variable which is spatially correlated and should be independent of the gravitational effect of topography. However for each line $i, \Delta g_{1}^{(i)}=\tilde{\Delta}_{1}^{(i)}+b_{i}$ where $b_{i}$ is the along track bias and $\tilde{\Delta g_{1}^{(i)}} s$ is the spatially correlated zero mean random variable.

Figures $6.22 \mathrm{a}$ shows the $8 \mathrm{~m}$ DEM filtered to a wavelength of $9 \mathrm{~km}$ (approximately the minimum wavelength of EGM2008 expanded up to degree and order 2160 at mid latitudes) using 2D Gaussian window function to avoid any high frequency ringing, and figure $6.22 \mathrm{~b}$ shows the estimated terrain effect on a grid $5000 \mathrm{~m}$ above the reference ellipsoid. Figures $6.23 \mathrm{a}$ and $6.23 \mathrm{~b}$ show the EGM2008 gravity anomaly at $5000 \mathrm{~m}$ above the reference ellipsoid before and after the terrain correction is applied. Figures 6.24a and $6.24 \mathrm{~b}$ show the terrain corrected EGM2008 along the flight lines and the residual gravity signal $\Delta g_{1}$. 

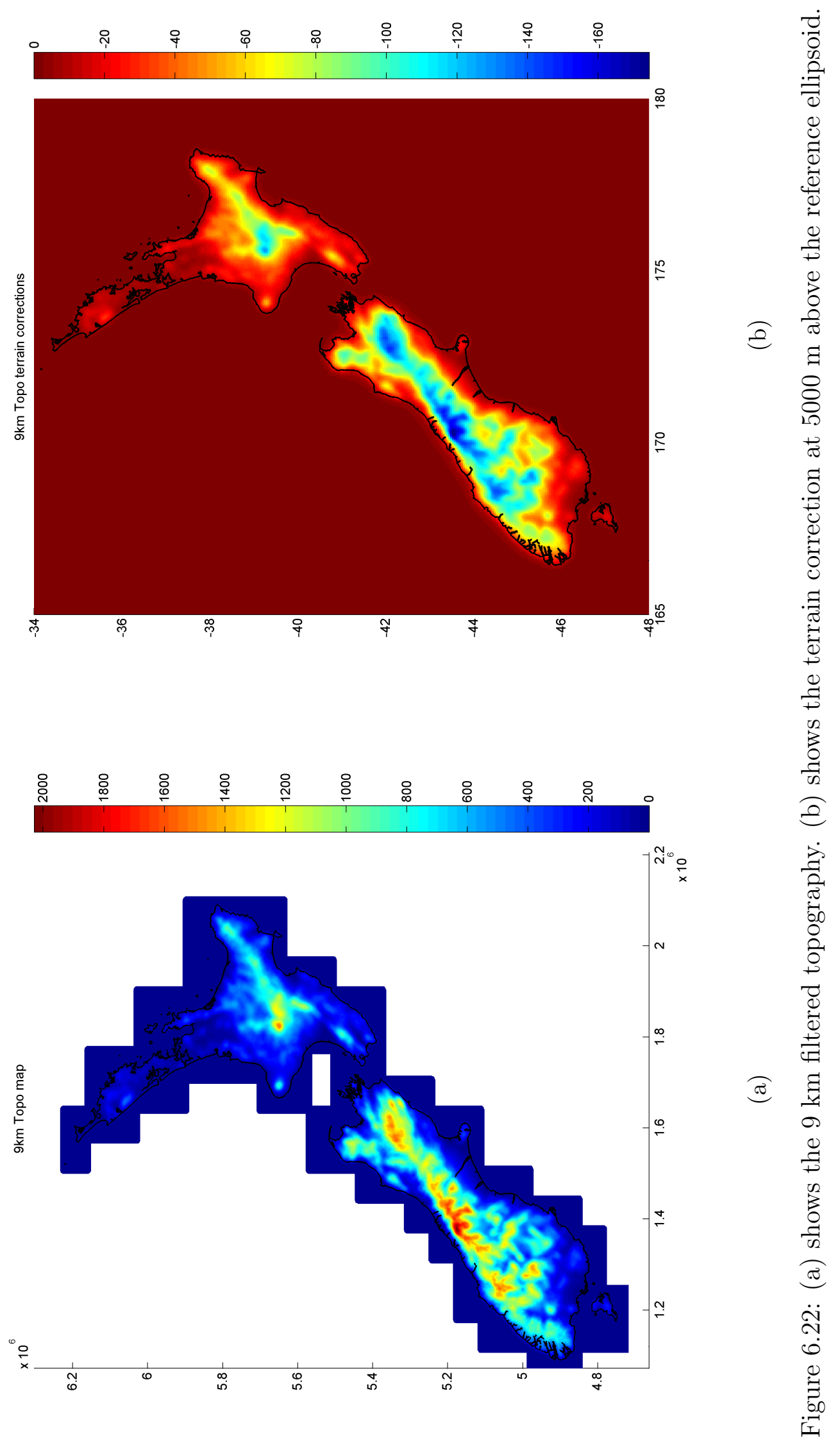

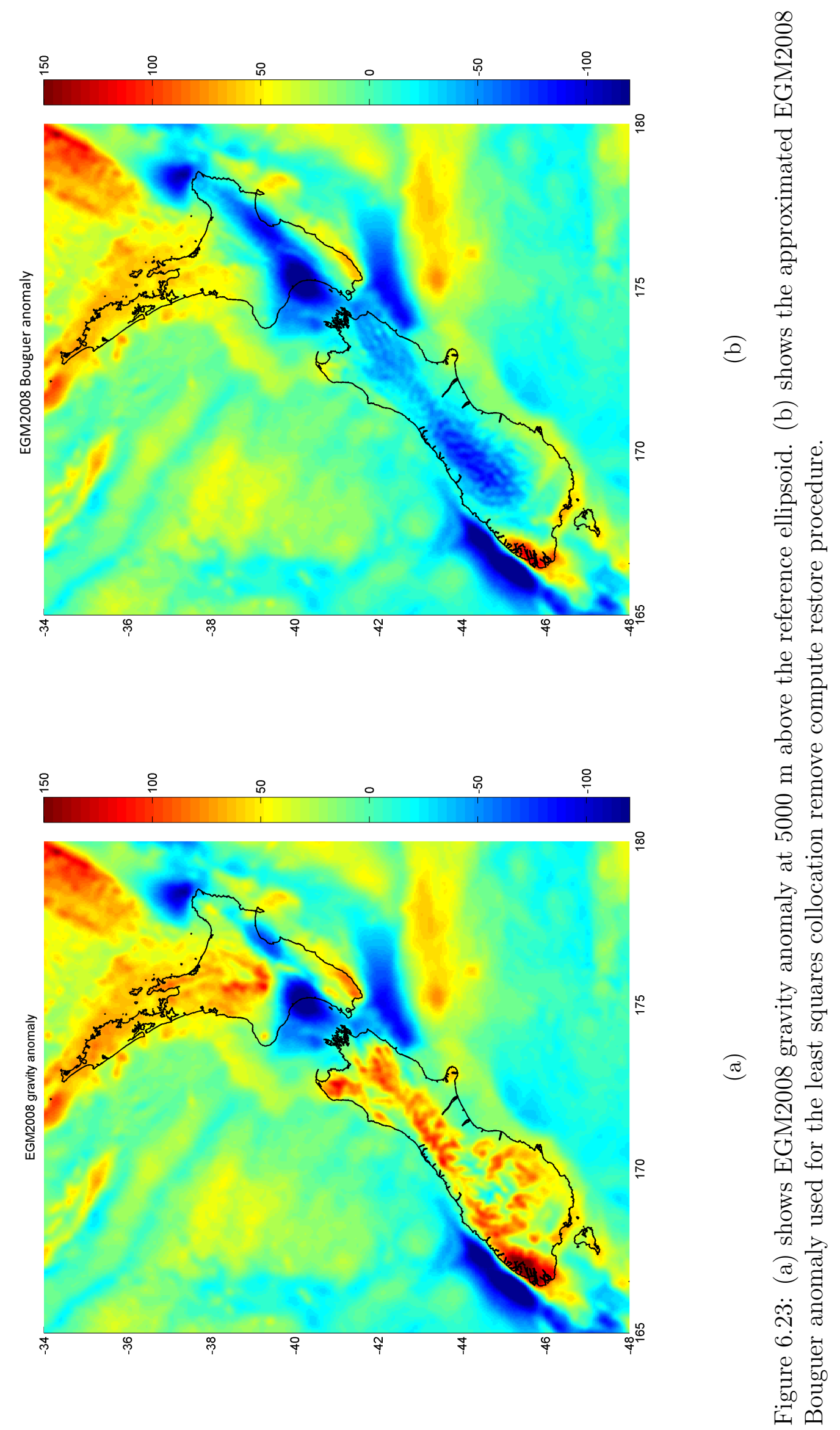

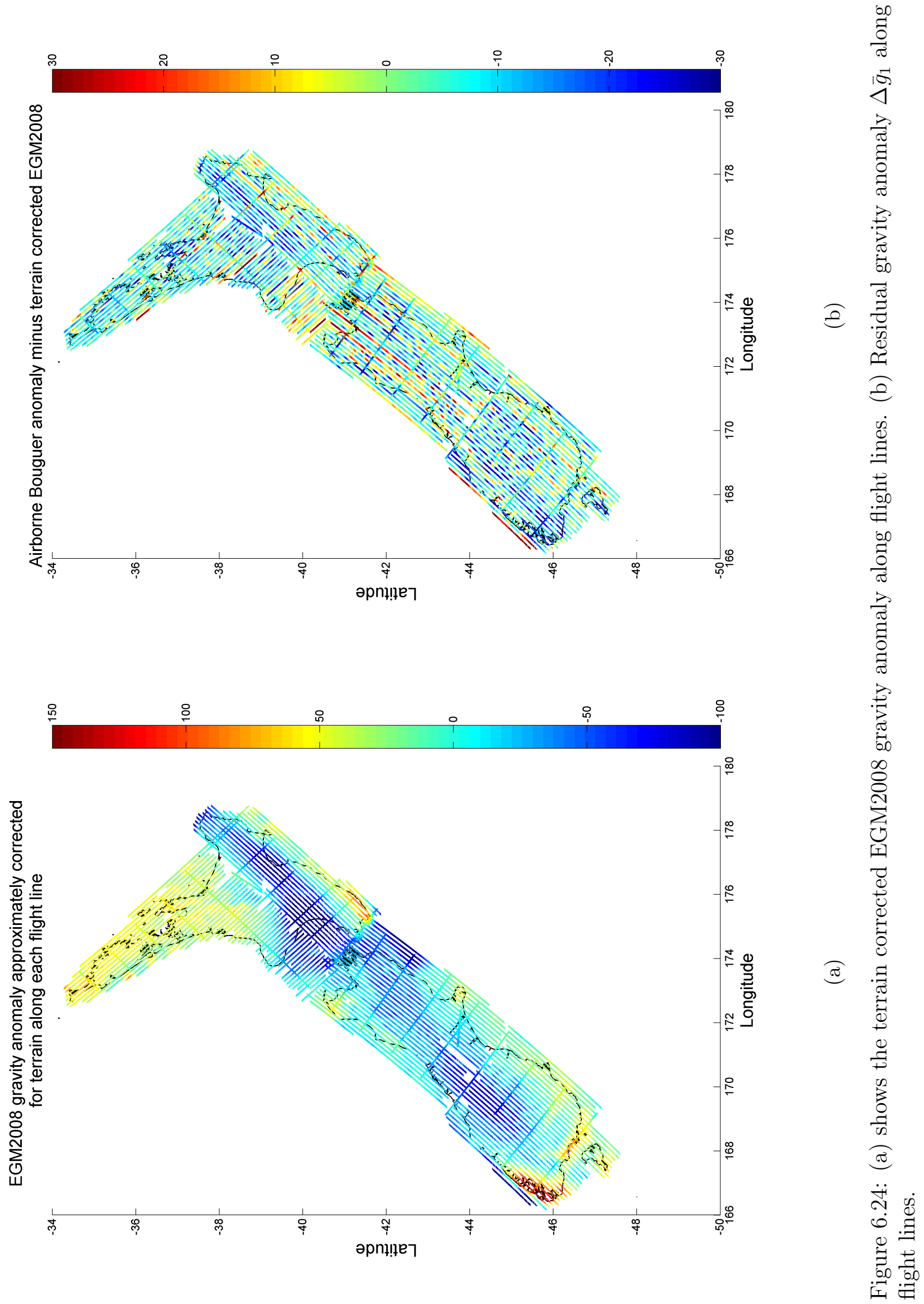
Step 5: Calculating the empirical covariance of the residual signal and fitting a covariance model: The empirical spatial covariance of the residual gravity data, here denoted $\Delta g$ is a function of distance. To compute it, the latitude and longitude coordinates must first be transformed to NZTM eastings and northings $x$ and $y$, the covariance values can be then calculated by equation 6.24 .

$$
\operatorname{Cov}\left(\hat{s}_{k}\right)=\sum_{j=1}^{j=N} \sum_{i: \sqrt{\left(x_{i}-x_{j}\right)^{2}+\left(y_{i}-y_{j}\right)^{2}} \in \hat{s}_{k}} \frac{\Delta g_{i} \Delta g_{j}}{n_{k}}
$$

where $N$ is the total number of residual gravity data, $\hat{s}_{k}$ is a radial "bin" with the range $[(k-1) s, k s]$ so that $s$ is the width of the "bin", and $n_{k}$ is the sum over $j$ of the number of points in each radial "bin" around the points $\left(x_{j}, y_{j}\right)$.

$\operatorname{Cov}\left(\hat{s}_{k}\right)$, (seen in figure 6.25 marked as blue circles), has been determined for the residual airborne gravity signal shown in figure $6.24 \mathrm{~b}$ with radial step sizes of $s=250 \mathrm{~m}$.

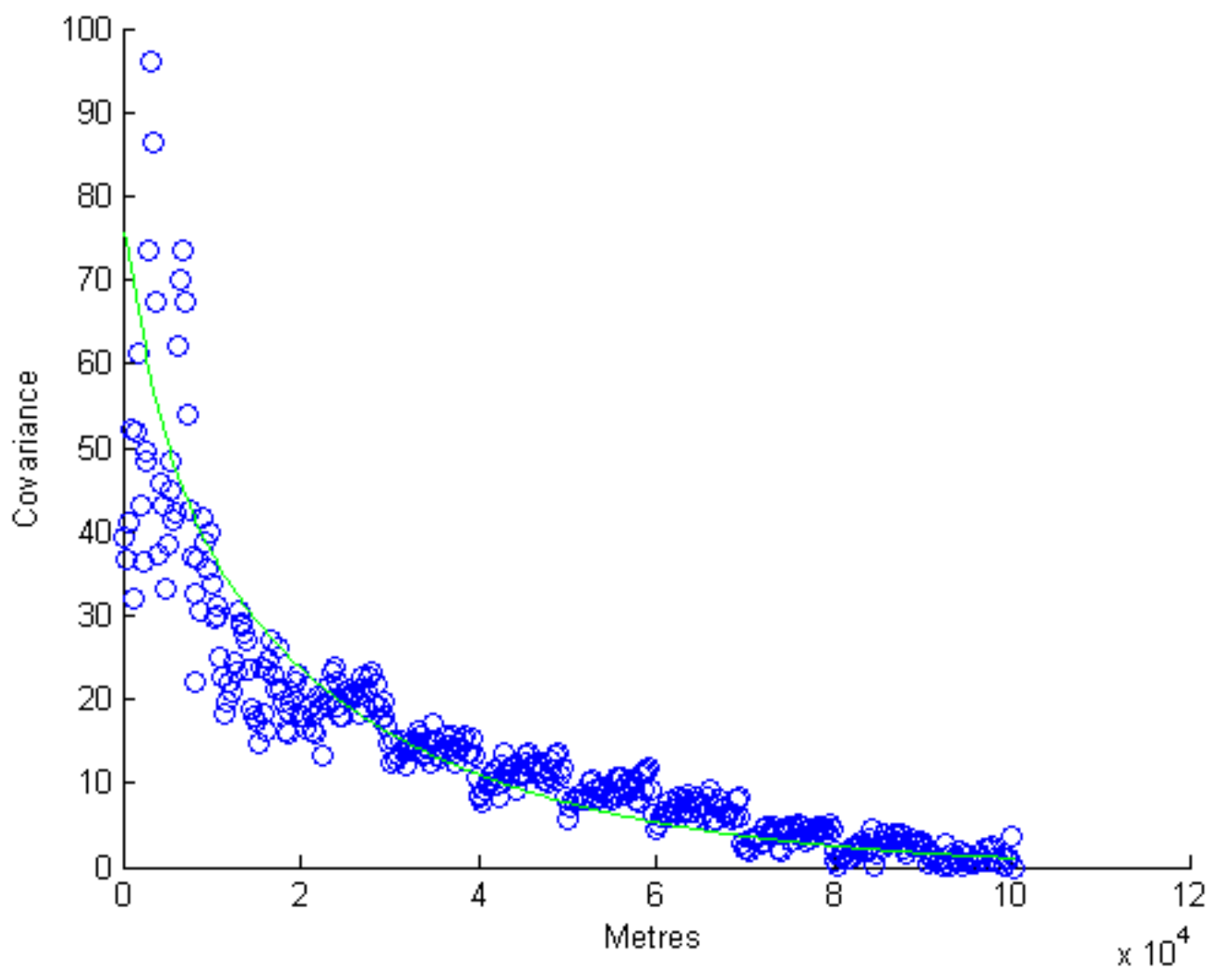

Figure 6.25: Empirical covariance is shown in blue and the fitted logarithmic covariance function is shown in green. 
A Forsberg (1987) covariance model of the form of equation 6.25 was then fitted to the empirical covariance values. The choice of this covariance model over multiple others is justified in section 7.1.

$$
C=-f \sum_{k=0}^{k=3} \alpha_{k} \log \left(D_{k}+\sqrt{s^{2}+\left(D_{k}+h_{1}+h_{2}\right)^{2}}\right)
$$

with

$$
f=C_{0} \log \left(\frac{(D+T)^{3}(D+3 T)}{D(D+2 T)^{3}}\right)
$$

here $h_{1}$ and $h_{2}$ are taken to be zero, $s$ distance in the $x$-y aircraft (i.e. the x-axis in figure 6.25), $\alpha_{k}=1,-3,3,-1$ and $D_{k}=D+k T$. The parameters $D, T$, and $C_{0}$ were varied over a range of values, and the model was evaluated at successive intervals of $s=0,125,375,625, \ldots \mathrm{m}$ and compared to the empirical covariances. An optimal fit was obtained with $C_{0}=75.8 \mathrm{mGal}, D=9.5 \mathrm{~km}$ and $T=48.5 \mathrm{~km}$. This can be seen by the green line in figure 6.25 .

Step 6: calculating the covariance matrix $C_{g g}$, bias coefficient matrix $A$ and noise matrix $N$ : For $n$ residuals gravity observations $\Delta g_{i}$ known at locations in 3D Euclidean space $x_{i}, y_{i}, z_{i}$ (with $i=1,2,3,4, . ., n$ ), the covariance matrix $C_{g g}$ is an $n \times n$ matrix.

An element in row $i$ column $j$ of $C_{g g}$ is determined as follows,

$$
C\left(\Delta g_{i}, \Delta g_{j}\right)=-f \sum_{k=0}^{k=3} \alpha_{k} \log \left(D_{k}+\sqrt{\left(x_{i}-x_{j}\right)^{2}+\left(y_{i}-y_{j}\right)^{2}+\left(D_{k}+z_{i}+z_{j}\right)^{2}}\right)
$$

where $C_{0}=75.8 \mathrm{mGal}, D=9.5 \mathrm{~km}$ and $T=48.5 \mathrm{~km}$ and $f$ is calculated using equation 6.26 .

Each residual gravity observation $\Delta g_{i}$ was collected along 1 of 197 different flight lines $k . A$ is an $n \times k$ matrix where for a row $i=1,2,3, . . n$ and column $k=1,2,3, \ldots 197 A_{i, k}$ is determined as to be either a 1 or a 0 as follows,

$$
\begin{array}{rr}
A_{i, k}=1 & \text { if } \Delta g_{i} \text { is a measurement along on flight line } k \\
A_{i, k}=0 & \text { otherwise }
\end{array}
$$

Here, the matrix $N$ of equation 6.15 is a diagonal matrix with values on the diagonal which corresponds to the uncorrelated noise level variance in the residual gravity observations. It has a regularization effect and the noise level variance value has been taken to be $9 \mathrm{mGal}^{2} .9 \mathrm{mGal}^{2}$ is in agreement with the derived accuracy of the data given in section 6.6. 
Step 7: practical considerations and results of solving equation 6.15 for $b$ : Figure 6.25 demonstrates that the covariance function with the determined parameters quickly decays to zero for $s>100 \mathrm{~km}$. The number of residual gravity anomalies is of the order $10^{6}$ which makes the matrices $C_{g g}$ and $N$ impractically large.

The bias calculation has been performed in Matlab which has the ability to only store non zero elements of large sparse matrices, for this reason $C_{g g}$ and $N$ have been stored as sparse matrices with $C\left(\Delta g_{i}, \Delta g_{j}\right)$ set exactly to zero for all $s_{i}=\sqrt{\left(x_{i}-x_{j}\right)^{2}+\left(y_{i}-y_{j}\right)^{2}}>100 k m$.

Using the sparse matrices $C_{g g}$ and $N$, matrix $A$ and a vector of the residual gravity anomalies $\Delta g$, the track biases, $b$ were then solved for using equation 6.15 where $b$ is a $197 \times 1$ vector of constants which correspond to the track bias for each of the 197 flight lines. If the residual gravity observations were uncorrelated spatially and "noise free" equation 6.29 would reduce to

$$
b=\left(A^{T}(I)^{-1} A\right)^{-1} A^{T}(I)^{-1} \Delta g=\left(A^{T} A\right)^{-1} A^{T} \Delta g,
$$

which is just a least squares solution for the average value of the residual gravity vector along each track. The inclusion of the matrix $C_{g g}$ ensures that spatially correlated signal in the residual gravity observations does not influence the estimated biases $b$.

Figure $6.26 \mathrm{a}$ shows the calculated biases $b$ along each flight line and figure $6.26 \mathrm{~b}$ shows the empirical distribution of the biases which has a standard deviation of around $5 \mathrm{mGal}$.

Figures $6.27 \mathrm{a}$ and $6.27 \mathrm{~b}$ show the residual gravity signal before and after the bias adjustment and figures $6.28 \mathrm{a}$ and $6.28 \mathrm{~b}$ shows the airborne gravity Bouguer anomaly before and after the bias adjustment.

The bias adjustment improves the internal consistency of the gravity anomalies which is demonstrated by the reduced cross-over error and improved agreement of the airborne data with the terrestrial gravity data as discussed in section 6.6. These improvements indicate the bias adjustment is appropriate and necessary. 


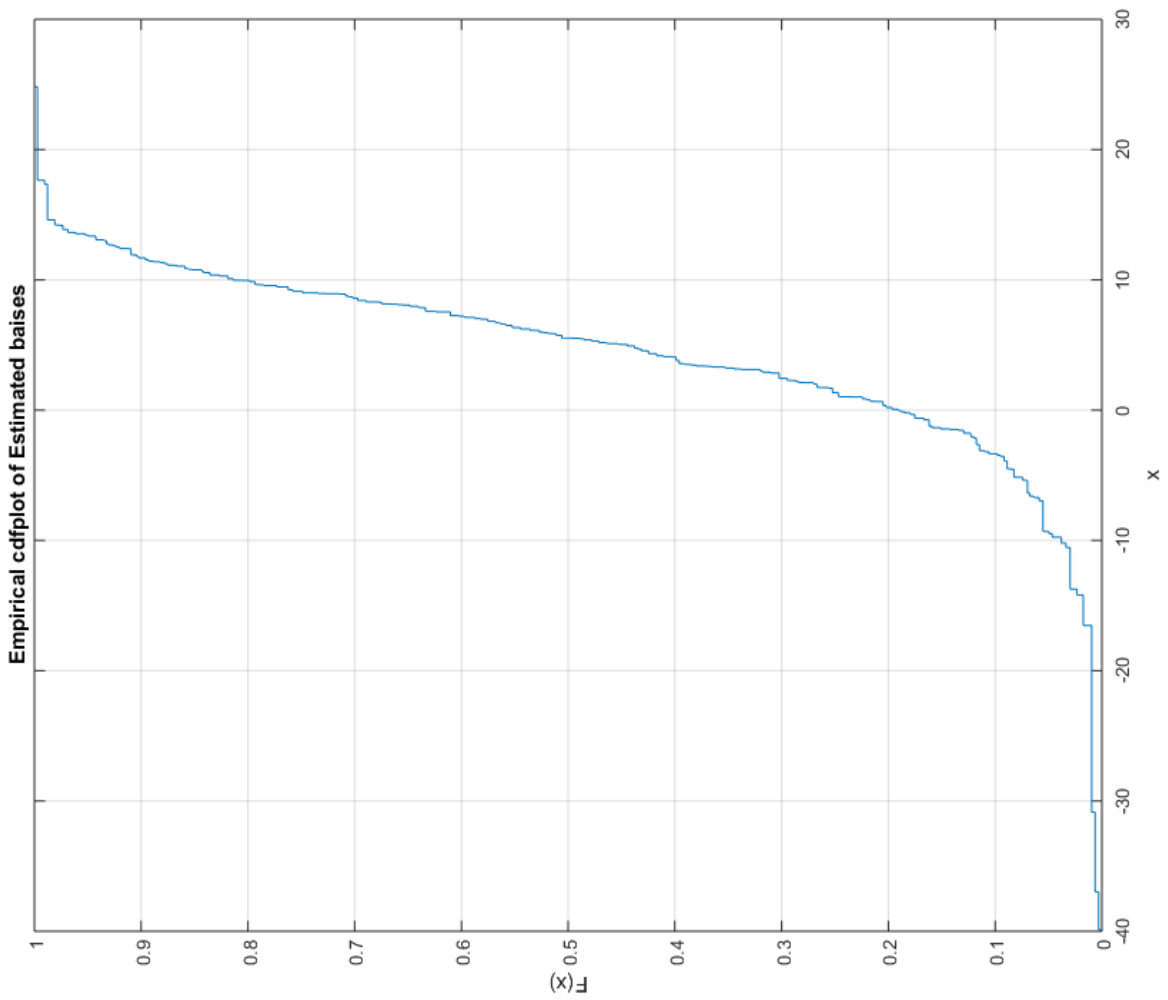

$\frac{9}{4}$

पै

물

岂

त्ठี艹

곤

อ

$\frac{5}{n}$

อ

苟

$\Xi$

.9.

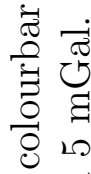

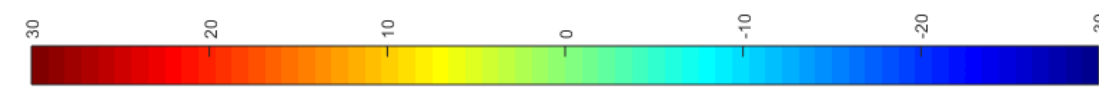

E

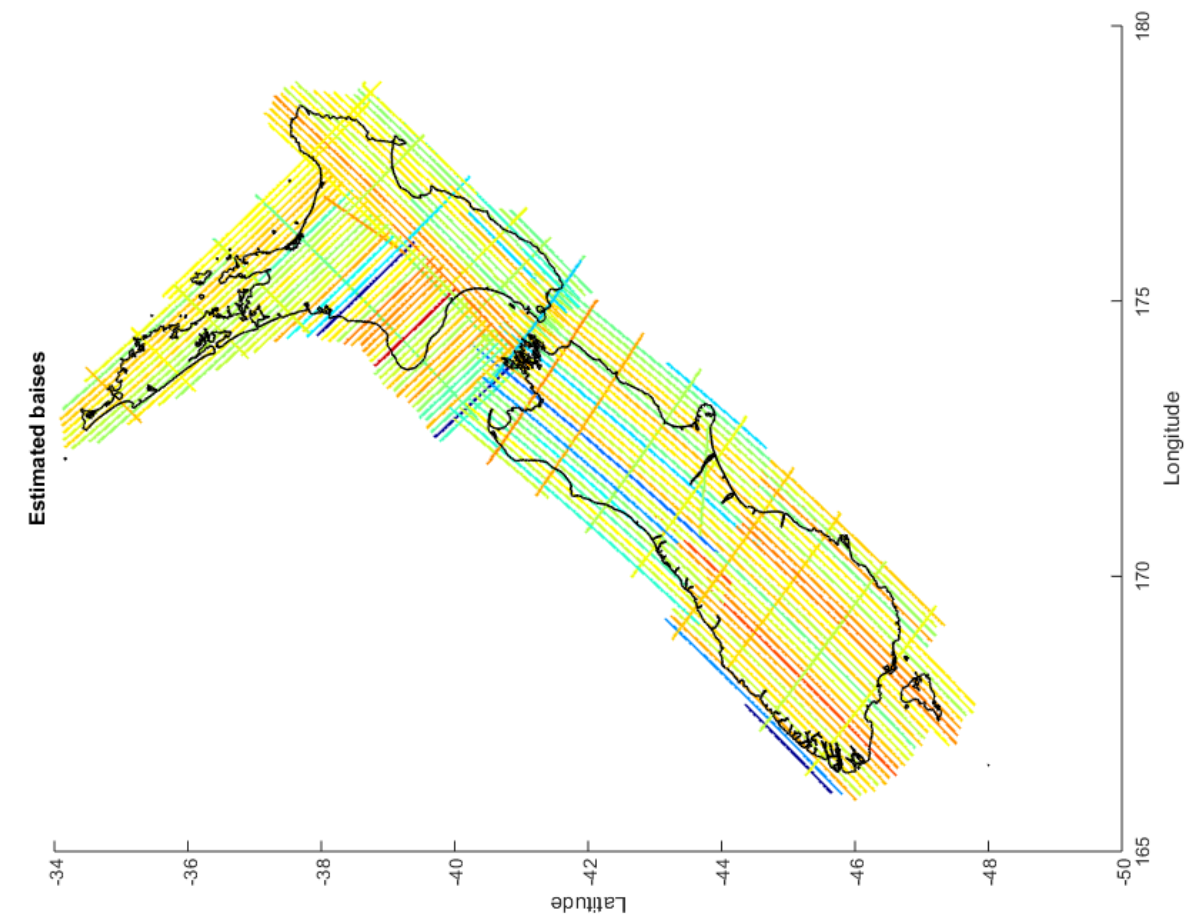

فำ ํ. 어 范 


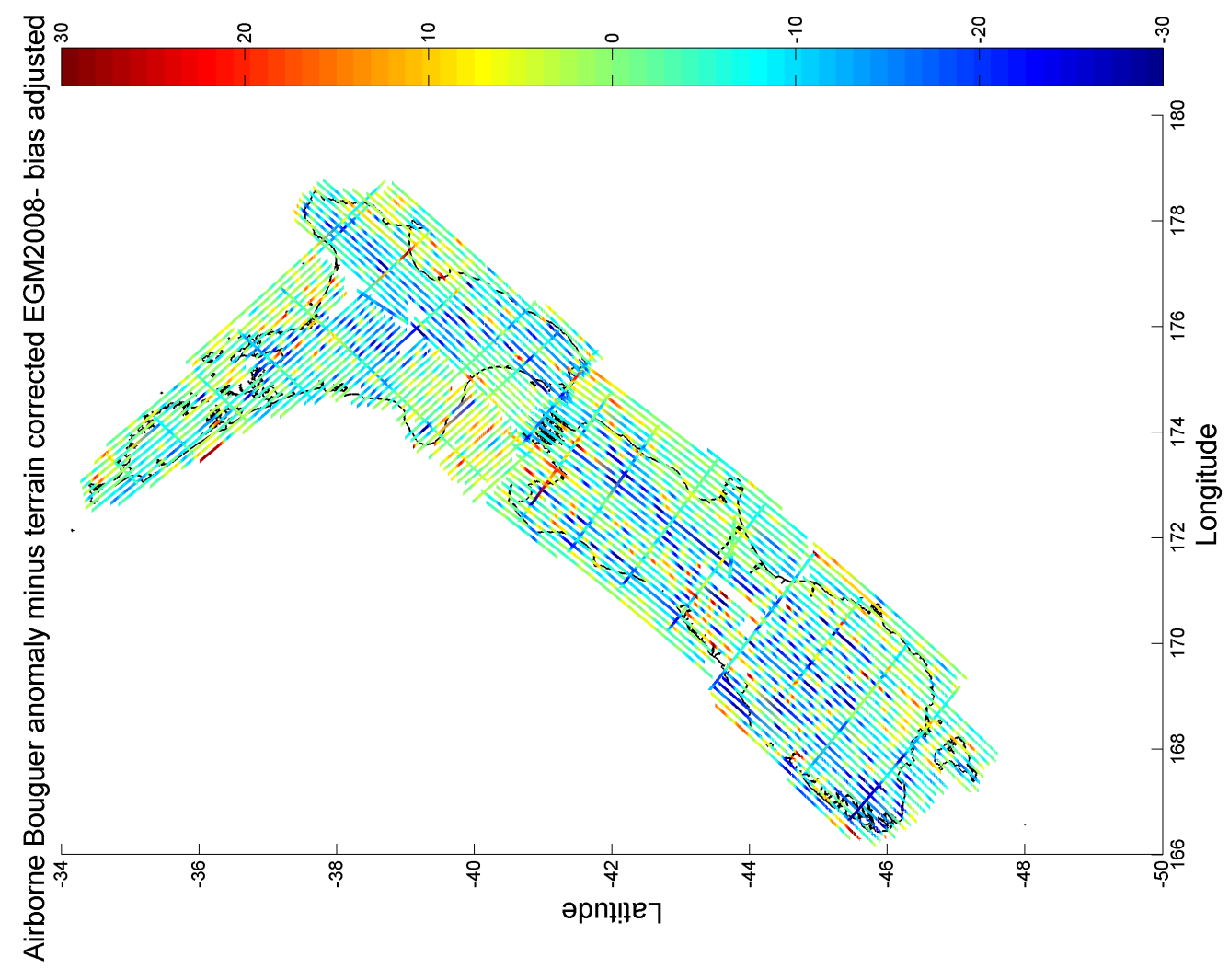

皇

e

0

हुं

$\exists$

. 19

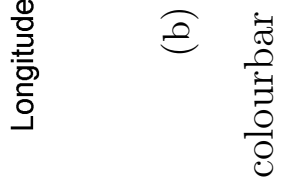

E

高

?]

건

苟
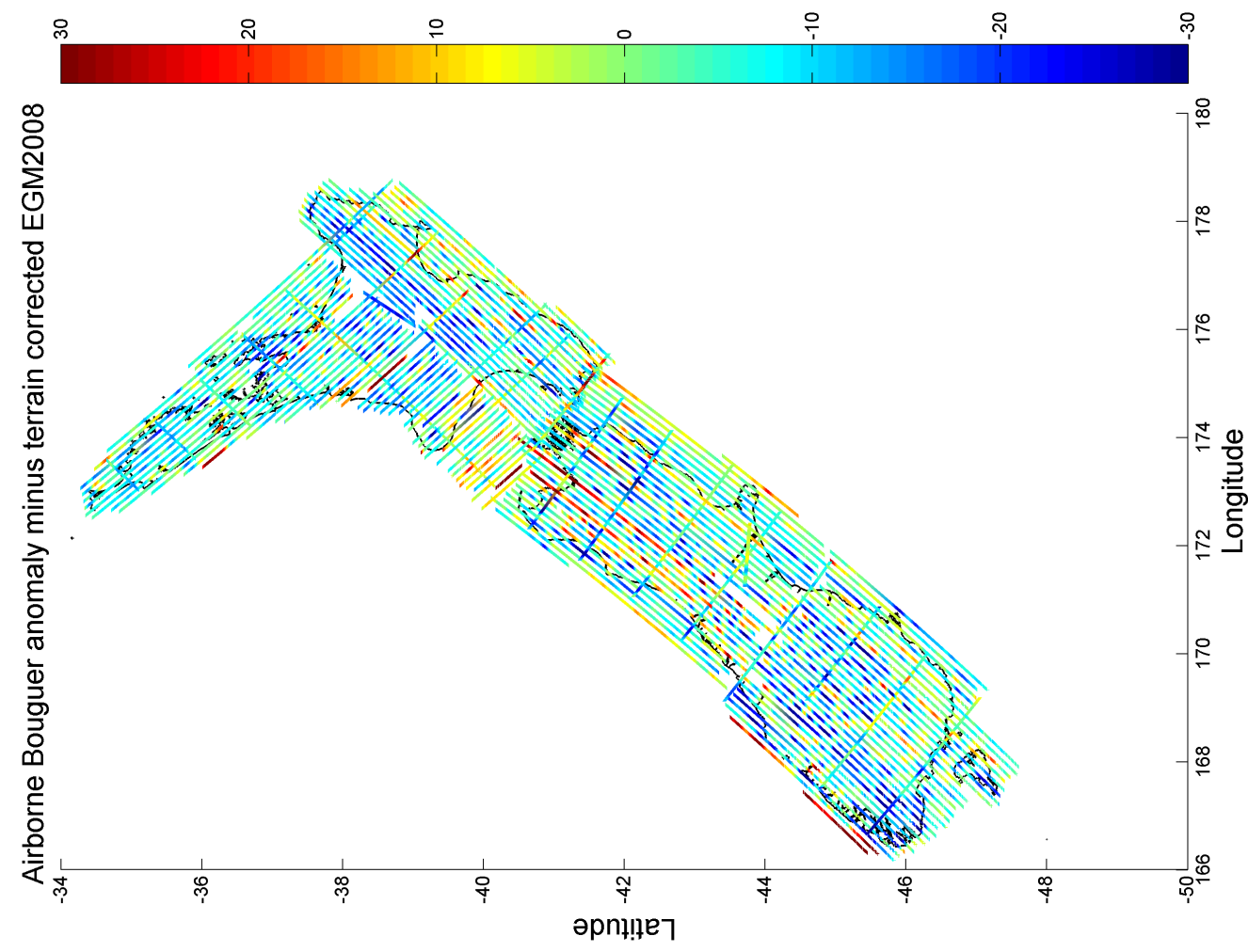

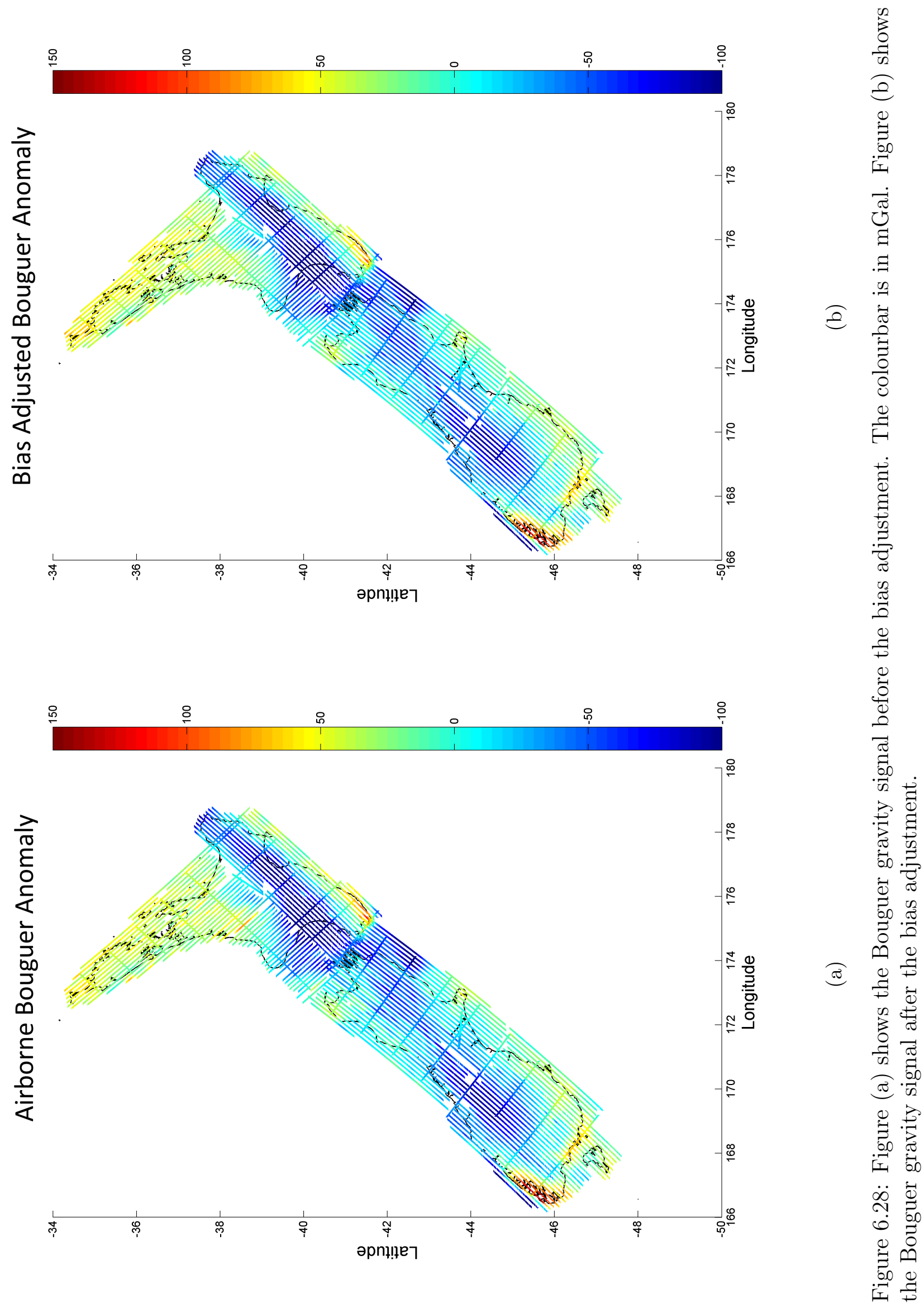


\subsection{Accuracy assessment}

The accuracy of the data has been assessed in 3 ways.

1. The difference in gravity anomalies at the intersection points of flight lines has been assessed.

These differences are shown to be sensitive to the along track filter parameter, the anisotropic terrain effects and the varying elevation of the flight lines. These points are explored by synthesizing flight line data using the EGM2008 global gravity model and applying the same along track filtering as used for the airborne data.

Over all the bias adjusted data had significantly smaller intersection point differences than before the bias adjustment. This indicates the bias adjustment improves the internal consistency of the data.

2. The airborne data are downward continued to the topographic surface and compared with all of the terrestrial gravity measurements discussed in section 4.1, this comparison is assessed before and after the bias adjustment. The bias adjustment had a significantly better agreement with the terrestrial than without the adjustment this indicates the bias adjustment is appropriate.

3. The difference in gravity anomalies for repeated flights along the calibration lines is assessed. Also, along these lines terrestrial gravity measurements have been obtained and so they are compared to the airborne data.

\subsubsection{Cross-over error}

Let $f_{j}, j=1,2,3, \ldots . J$ and $f_{k}, k=1,2,3, \ldots K$ be two distinct sets of flight lines which run perpendicular to each other. Let $\mathbf{x}_{j, k}$ be the intersection point of lines $f_{j}$ and $f_{k}$ with $\Delta g_{j, k}$ the true gravity anomaly at that point.

If $\tilde{\Delta} g_{j, k}$ and $\tilde{\Delta} \psi_{j, k}$ are the measured gravity anomaly along lines $f_{j}$ and $f_{k}$ respectively at the point $\mathbf{x}_{j, k}$,

$$
\tilde{\Delta} g_{j, k}=\Delta g_{j, k}+\mu_{j, k}
$$

and

$$
\tilde{\Delta} \psi_{j, k}=\Delta g_{j, k}+v_{j, k}
$$

then the cross-over difference, $C_{j, k}$, is given by,

$$
\tilde{\Delta} g_{j, k}-\tilde{\Delta} \psi_{j, k}=\mu_{j, k}-v_{j, k}=C_{j, k} .
$$

The cross-over error is then given by the standard deviation of $C_{j, k} \forall j, k$. 
The cross-over differences have been calculated for all intersection points, before and after the along track bias correction, for all airborne gravity data where there has not been a zero weight assigned. These points are shown in figure 6.29.

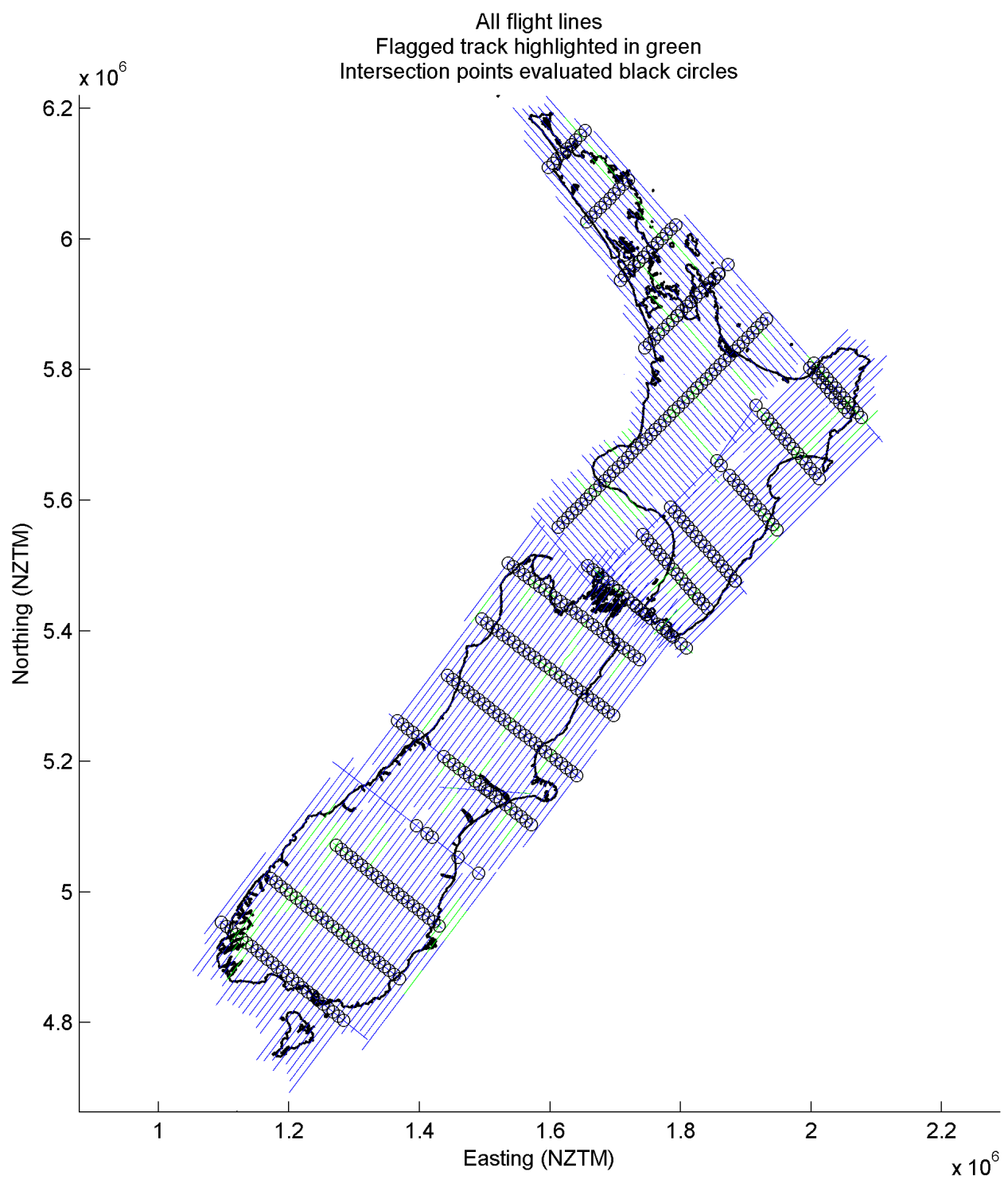

Figure 6.29: Flight lines and tie line intersection points.

The free air anomaly data before and after the bias correction have been filtered with a varied Gaussian filter parameter and the results of the cross-over error have been recorded in table 6.1. The cross-over difference is smallest with a filter parameter of 120 seconds before the bias adjustment is applied. With an average flight speed of 130 knots and a measurement made at $1 \mathrm{~Hz}, 120$ seconds corresponds to around $8 \mathrm{~km}$ spatially. Figure 6.30 shows the empirical cdf plots of the cross-over difference before and after the bias adjustment for a 120 seconds filter. The standard deviation of the inner $68 \%$ of the distributions is $9.3 \mathrm{mGal}$ before bias adjustment and $7.3 \mathrm{mGal}$ after. 
Table 6.1: The filter sigma parameter in seconds and free air cross-over difference standard deviation (of the inner $68 \%$ following appendix B) before and after bias correction.

$\begin{array}{lll}\text { Filter Parameter } & \begin{array}{l}\text { Free air anomaly } \\ \text { cross-over std } 68 \%\end{array} & \begin{array}{l}\text { Cross-over } \\ \text { after bias correction std } 68 \%\end{array} \\ 10 & 149.46 & 149.87 \\ 20 & 31.97 & 32.01 \\ 30 & 17.65 & 16.61 \\ 40 & 14.71 & 12.98 \\ 50 & 12.48 & 11.65 \\ 60 & 11.67 & 10.26 \\ 70 & 11.07 & 9.421 \\ 80 & 10.46 & 8.572 \\ 90 & 10.12 & 7.858 \\ 100 & 9.705 & 7.307 \\ 110 & 9.404 & 7.175 \\ 120 & 9.362 & 7.313 \\ 130 & 9.763 & 7.438 \\ 140 & 10.35 & 7.445 \\ 150 & 11.02 & 7.481 \\ 160 & 11.44 & 7.652 \\ 170 & 11.99 & 7.796 \\ 180 & 12.69 & 8.091 \\ 190 & 13.09 & 8.402 \\ 200 & 13.45 & 8.654\end{array}$

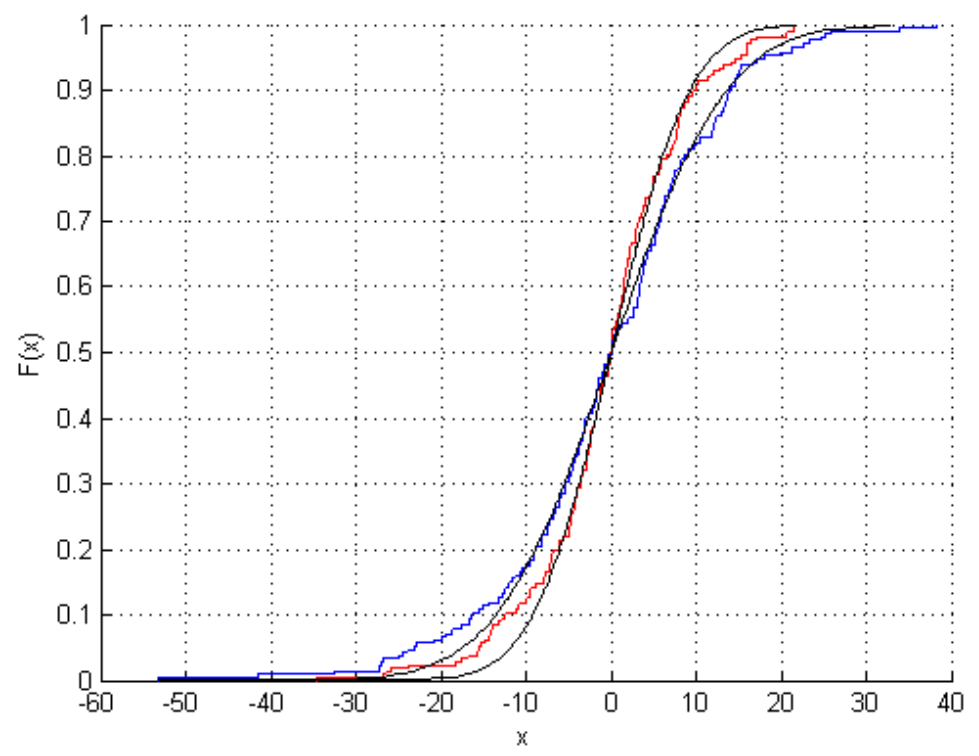

Figure 6.30: Empirical CDF plot of free air cross-over differences (in blue), Gaussian distribution fitted to the inner $68 \%$ has a standard deviation of $9.3 \mathrm{mGal}$ before bias adjustment and $7.3 \mathrm{mGal}$ after. 


\section{Systematic error in the cross-over differences.}

The comparison between the normal flight lines and the cross tie lines is not strictly fair. This is due to the following two reasons.

1. The data have been along track filtered in the time domain (which is essentially a directional spatial filter). This is problematic as the gravitational effect of the topography and the underlying gravity field are anisotropic.

2. The flight lines were flown at a varying elevations.

Investigating the effect of the along track filtering and terrain on the crossover error. To investigate the effect of the along track filtering on the cross-over differences, the gravity anomaly along each flight line has been estimated using EGM2008.

When an along track filter is applied to the EGM2008 gravity anomaly data, the gravity values at intersection points of perpendicular flight lines diverge. To illustrate this, figure 6.31 shows two flight lines on the south Island, 25 (North-East, South-West) and 47 (South-East , NorthWest), and their along track gravity anomalies.
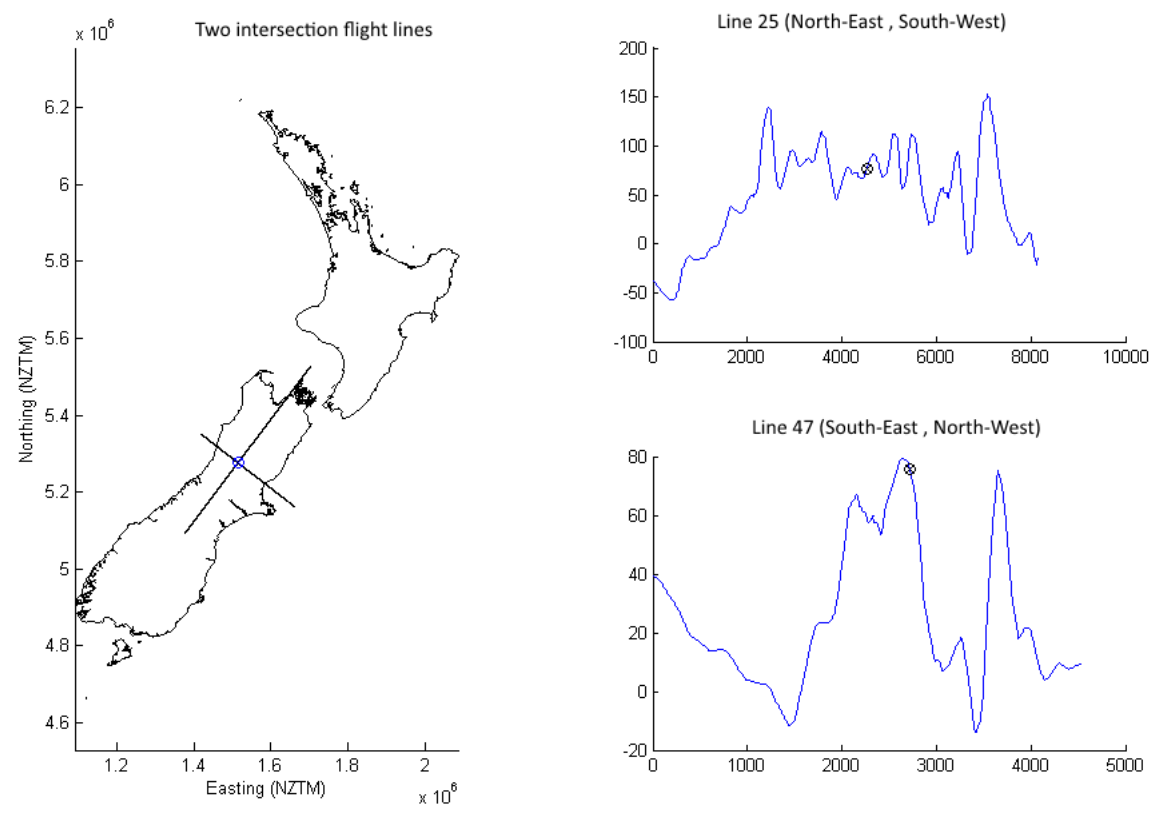

Figure 6.31: Flight lines 25 (North-East, South-West) and 47 (South-East, North-West) on the South Island and free air anomaly profiles along each line and intersection point marked by a black 'o' .

The topography in that part of the country in general runs in the North-East, South-West direction. When filtering along the line, the gravitational effect of the topography is smoothed out in the direction of the line only. For this reason as the filter width increases so does the difference between the gravity anomaly at the intersection point along both lines. 


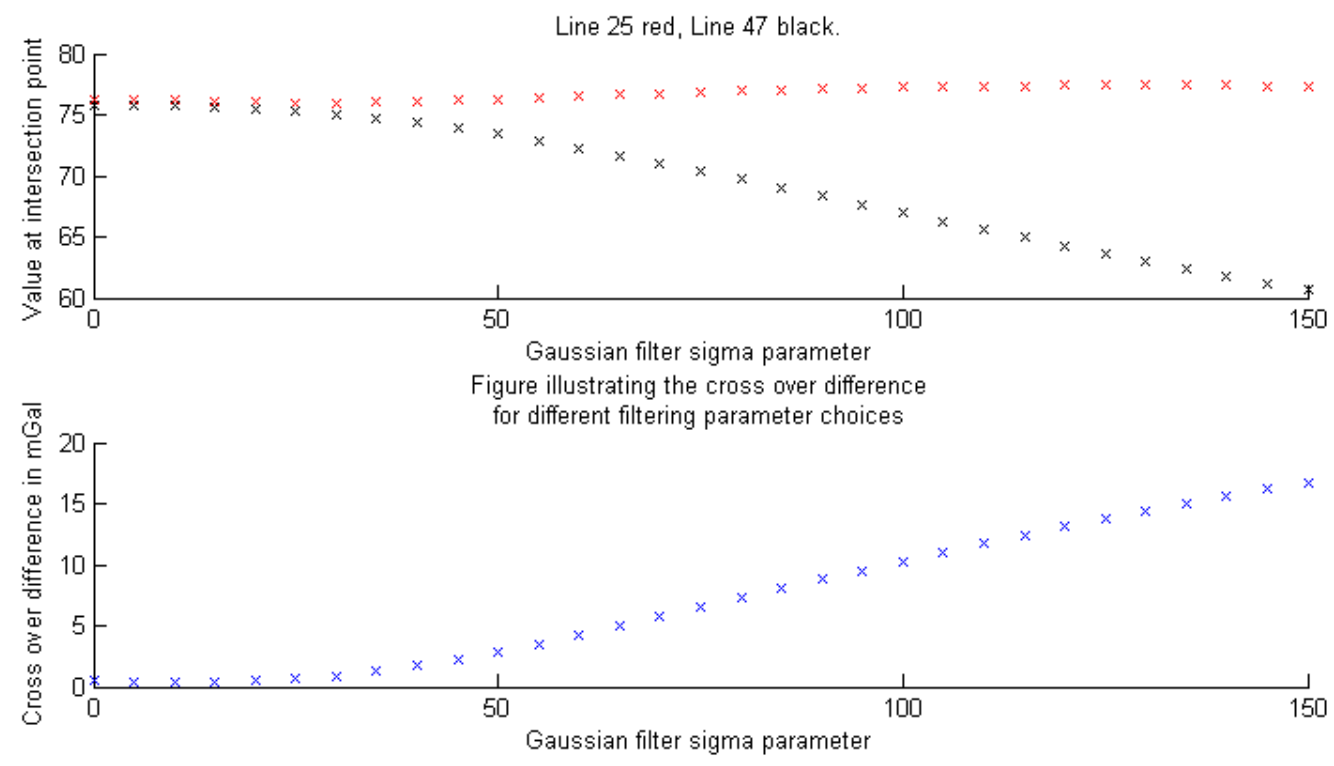

Figure 6.32: Flight lines 25 (North-East, South-West) and 47 (South-East, North-West) value at intersection point (top) and the difference (bottom).

Figure 6.33 shows the empirical cdf plot of the cross-over differences for the synthetic data with a filter width of 120 seconds. The distribution is leptokurtotic so a Gaussian cumulative density function was fitted to the inner $68 \%$ of the distribution, the fitted distribution has a standard deviation of $4.9 \mathrm{mGal}$.

Table 6.2 shows the standard deviation of the inner $68 \%$ of all of the cross-over differences for a range of filter parameters. It can be seen clearly that the standard deviation increases with the filter width and the internal consistency of the synthesized flight lines decays.

The large cross-over differences are typically located at the local minima and maxima of the along track gravity profile since the filtering will suppress sharp peaks and troughs. This is demonstrated in the figure 6.34, (using the synthetic EGM2008 data) where the gravity profile of the tie line is shown on the right in red with the blue circles the data extracted from the perpendicular across track flight lines at the intersection points.

Most of the high frequency content of the gravity field is due to topography. By performing a terrain correction (as discussed in section 6.5) to the synthetic data before filtering many of the peaks and troughs are removed. This can be seen in figure 6.35 of the terrain corrected gravity profile over the same flight line.

Performing the terrain correction prior to the along track filtering reduces the cross-over error at the intersection points compared to the free air anomaly. The standard deviation of the synthetic data cross-over difference for a range of parameters is shown in table 6.3. 
Table 6.2: Table of filter parameters and EGM2008 synthetic flight line cross-over difference standard deviations in $\mathrm{mGal}$.

$\begin{array}{ll}\text { Filter parameter (s) } & \begin{array}{l}\text { EGM2008 Free Air } \\ \text { Cross-Over std 68\% }\end{array} \\ 10 & 0.90 \\ 20 & 0.98 \\ 30 & 1.30 \\ 40 & 1.70 \\ 50 & 2.14 \\ 60 & 2.62 \\ 70 & 3.07 \\ 80 & 3.49 \\ 90 & 3.88 \\ 100 & 4.25 \\ 110 & 4.60 \\ 120 & 4.94 \\ 130 & 5.23 \\ 140 & 5.51 \\ 150 & 5.82 \\ 160 & 6.11 \\ 170 & 6.39 \\ 180 & 6.67 \\ 190 & 6.95 \\ 200 & 7.24\end{array}$

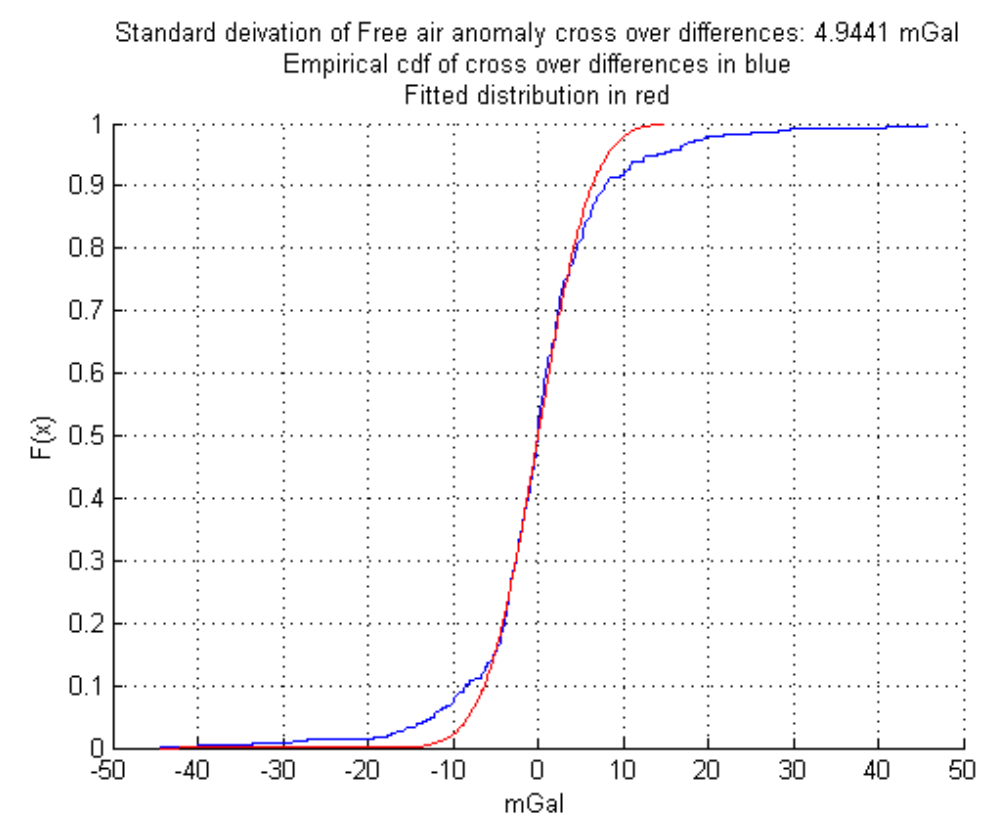

Figure 6.33: Empirical CDF of EGM2008 cross-over differences (in blue), fitted distribution (in red) with standard deviation of $4.94 \mathrm{mGal}$, with Gaussian filter parameter of 120 seconds. 

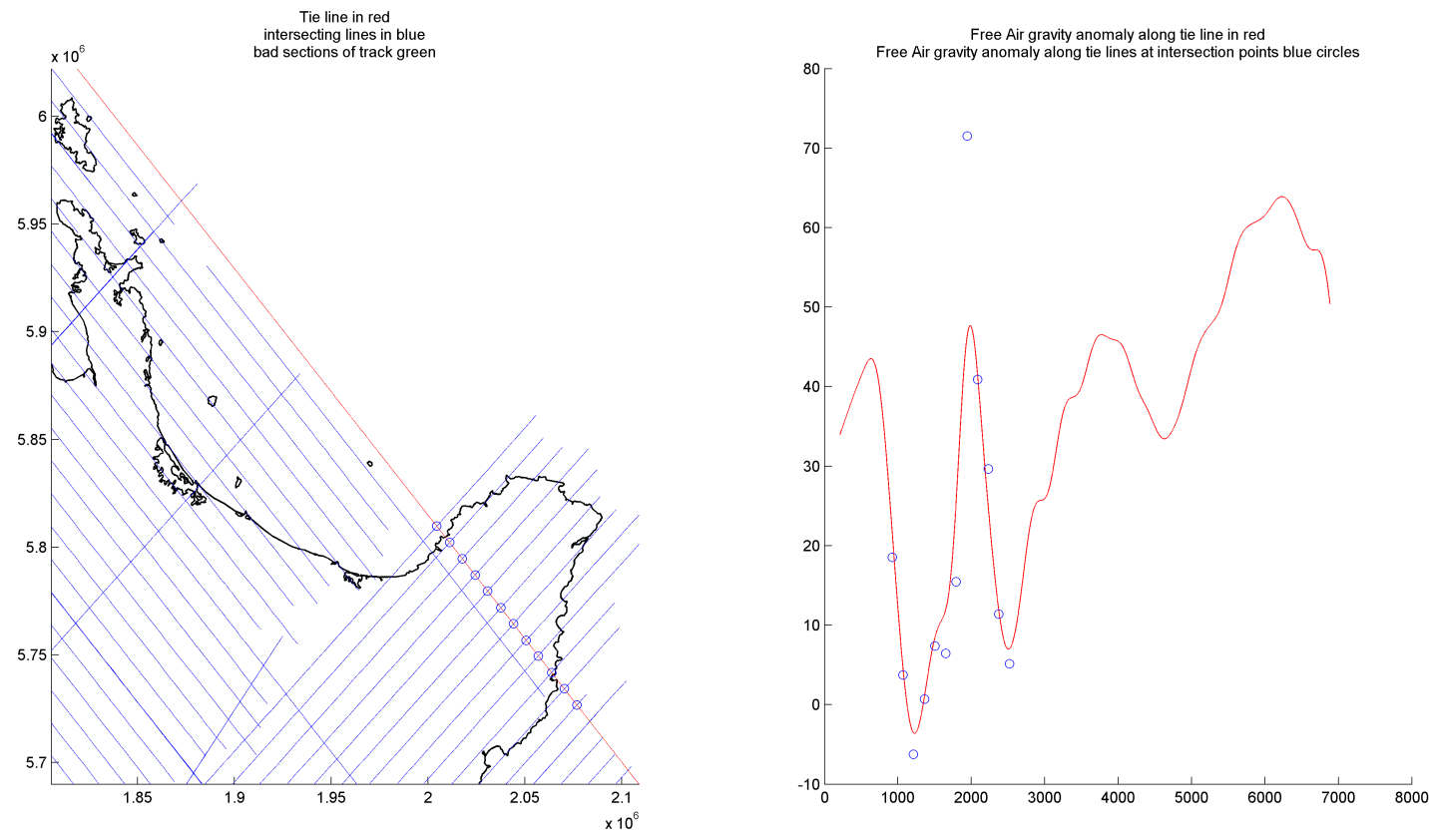

Figure 6.34: Left: Flight lines (blue), tie line (red) and intersection points (blue o's), Right: Tie line free air gravity profile in seconds along the line (red) and line values at intersection points (blue o's).
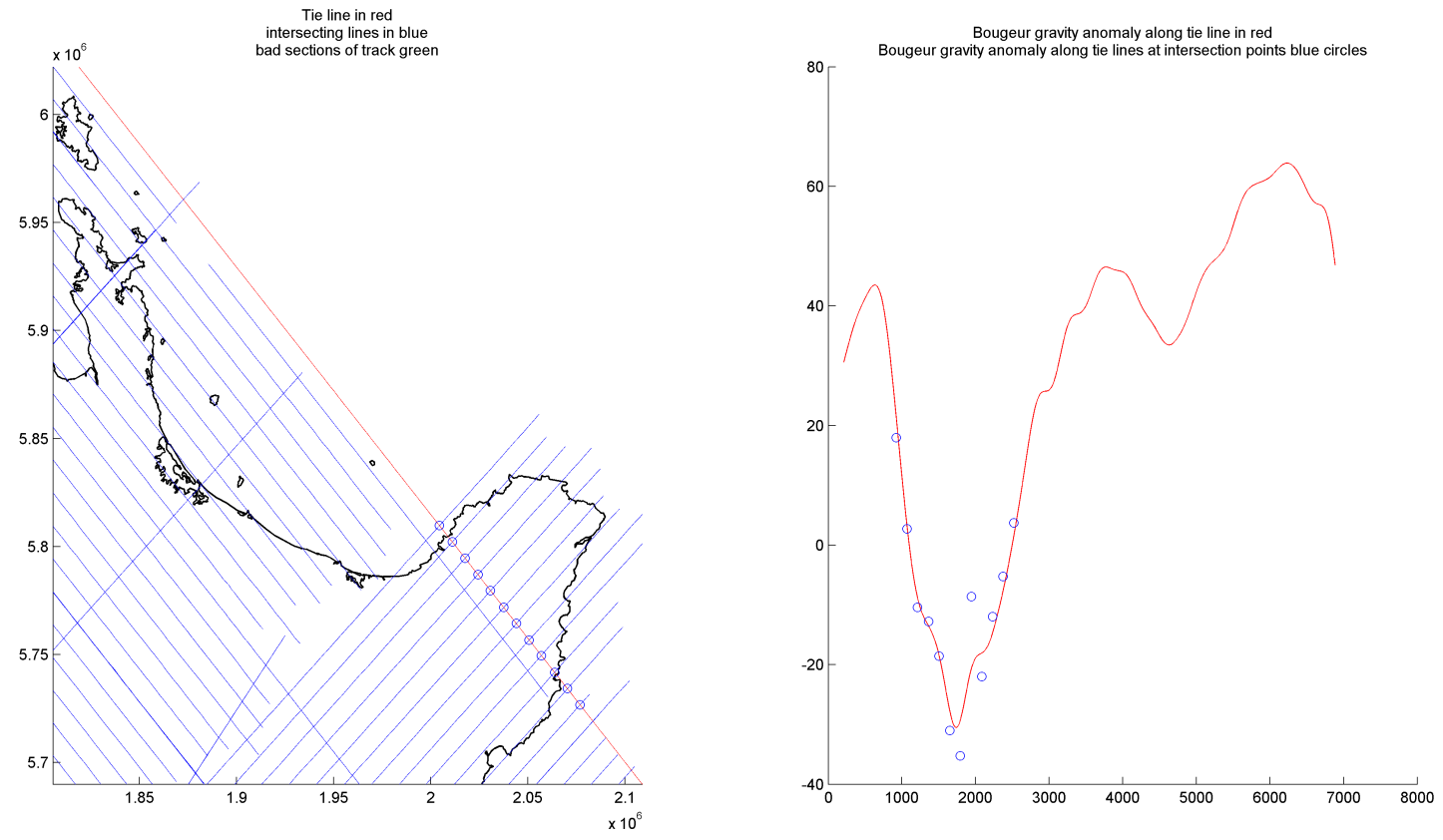

Figure 6.35: Left: Flight lines (blue), tie line (red) and intersection points (blue o's), Right: Tie line Bouguer gravity profile in seconds along the line (red) and line values at intersection points (blue o's). 
Table 6.3: Table of filter parameters and terrain corrected EGM2008 synthetic flight line cross-over difference standard deviations in mGal.
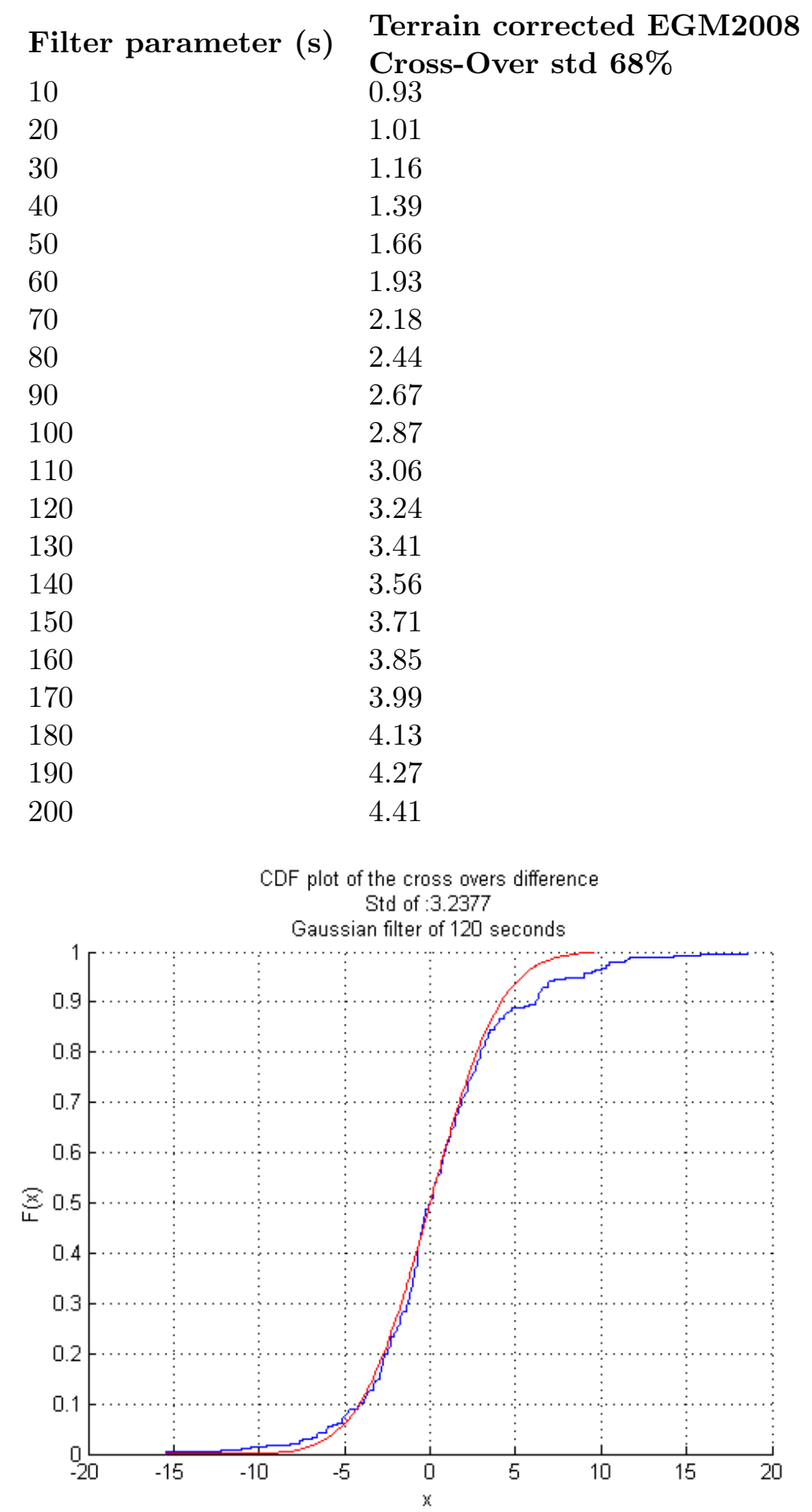

Figure 6.36: Empirical CDF plot of EGM2008 cross-over differences (in blue), Gaussian distribution fitted to the inner $68 \%$ has a standard deviation of $3.24 \mathrm{mGal}$. 
The cross-over error has similarly been calculate for the airborne Bouguer anomaly data. The standard deviation of the inner $68 \%$ of the cross-over difference for a range of filter parameters is shown in table 6.4 for both before and after the bias correction. The Bouguer anomaly crossover differences are distinctly smaller than those for the free air anomaly data.

As with the free air anomaly, prior to the cross-over adjustment a 120 second filter has the smallest cross-over difference standard deviation.

Figure 6.37 shows the empirical cdf of the cross-over differences for a filter parameter of 120 seconds before and after the bias correction. A Gaussian distribution has been fitted to the inner $68 \%$ of each and the standard deviations are $8.4 \mathrm{mGal}$ before bias adjustment and 6.3 mGal after.

Table 6.4: The filter sigma parameter in seconds and Bouguer cross-over difference standard deviation (estimated from the inner 68\%) before and after bias correction.

$\begin{array}{lll}\text { Filter Parameter } & \begin{array}{l}\text { Airborne Bouguer anomaly } \\ \text { cross-over std } 68 \%\end{array} & \begin{array}{l}\text { Cross-over } \\ \text { after bias correction std } 68 \% \\ 149.21\end{array} \\ 10 & 31.68 & 149.56 \\ 20 & 17.44 & 32.02 \\ 30 & 14.36 & 16.23 \\ 40 & 12.08 & 12.58 \\ 50 & 11.38 & 11.06 \\ 60 & 10.54 & 9.48 \\ 70 & 9.736 & 8.65 \\ 80 & 9.206 & 7.72 \\ 90 & 8.786 & 6.87 \\ 100 & 8.443 & 6.54 \\ 110 & 8.434 & 6.36 \\ 120 & 8.870 & 6.33 \\ 130 & 9.558 & 6.21 \\ 140 & 9.906 & 6.19 \\ 150 & 10.19 & 6.27 \\ 160 & 10.47 & 6.25 \\ 170 & 10.86 & 6.41 \\ 180 & 11.13 & 6.55 \\ 190 & 11.34 & 6.69 \\ 200 & & 6.76 \\ & & \end{array}$




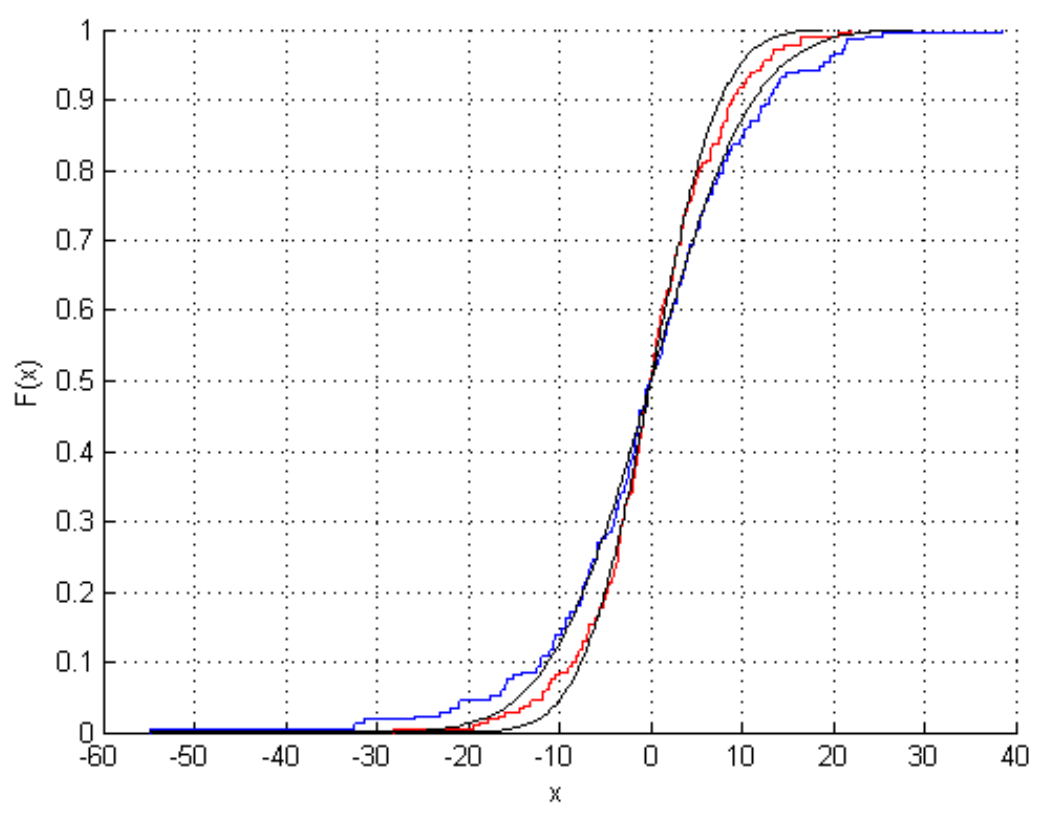

Figure 6.37: Empirical CDF plot of Bouguer cross-over differences (in blue), Gaussian distribution fitted to the inner $68 \%$ has a standard deviation of $8.4 \mathrm{mGal}$ before bias adjustment and $6.3 \mathrm{mGal}$ after.

For both the free air and Bouguer airborne gravity anomalies, an along track Gaussian filter $\sigma$ parameter of 120 seconds minimizes the standard deviation of cross-over differences before a bias adjustment is made.

The relationship between the improperly attenuated gravity signal due to the along track filter and the noise reduction can be seen by plotting the EGM2008 and airborne cross-over differences standard deviations against the filter parameter. Figures 6.38 and 6.39 appear to show that the bias corrected cross-over differences and EGM2008 cross-over differences converge. The EGM2008 cross-over difference standard deviation gives a good measure of the cross-over discrepancy level due to the filter parameter. The noise level in the airborne data can be estimated by taking the square root of the variance of the airborne cross-over differences minus the variance of the EGM2008 cross-over differences. i.e. for $\sigma_{k}^{2}$ the variance of the airborne crossover differences for some filter parameter $k$ and $\hat{\sigma}_{k}^{2}$ the variance of the EGM2008 cross-over differences, $\tilde{\sigma}_{k}$ the estimated noise level standard deviation is given by,

$$
\tilde{\sigma}_{k}=\sqrt{\sigma_{k}^{2}-\hat{\sigma}_{k}^{2}}
$$

The free air anomaly noise level $\tilde{\sigma}_{k}$ intersects with the EGM2008 cross-over difference standard deviation for a filter length of 120 seconds as can be seen in figure 6.40. This indicates any filter length greater than 120 seconds is likely to be attenuating more of the gravity signal than noise.

It has been seen that the along track filter strongly influences the cross-over differences. The along track filter is a directional filter which attenuates high frequency gravity anomalies along the flight lines. Most of the high frequency gravity signal is attributable to the topography. 


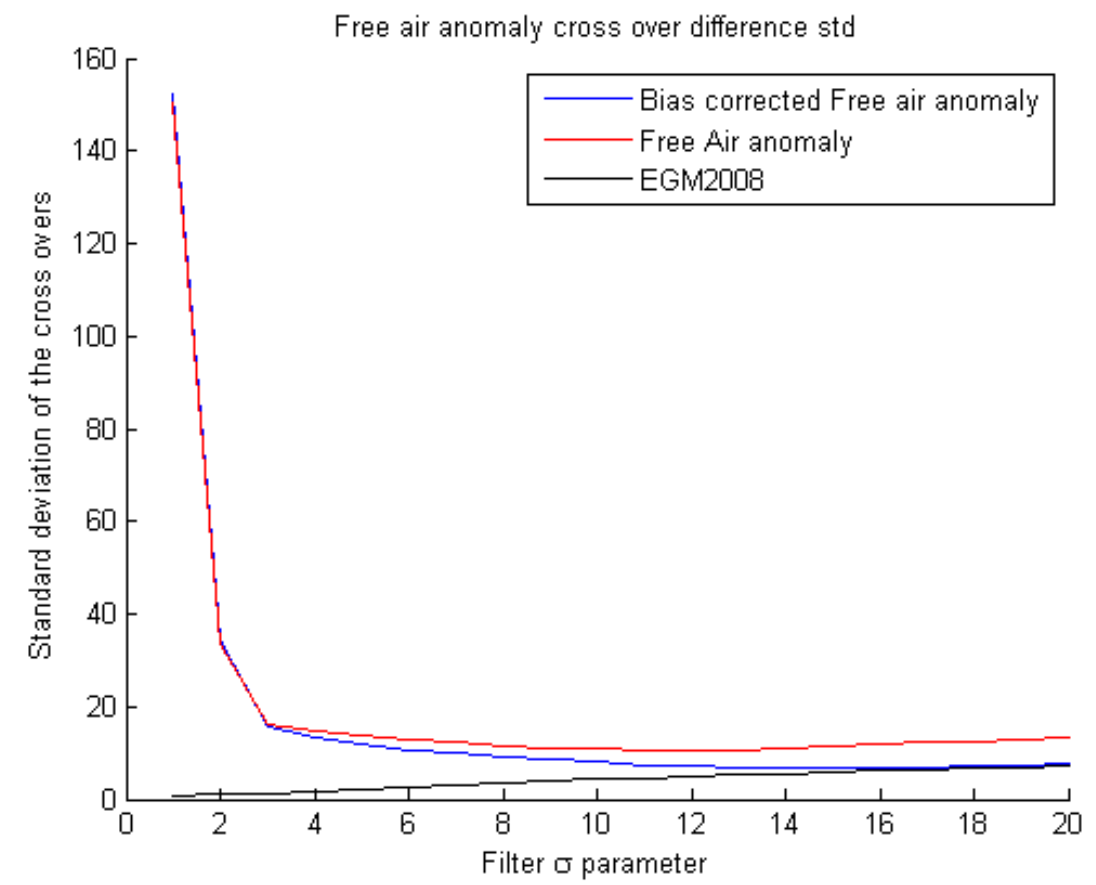

Figure 6.38: Standard deviation of the EGM2008, airborne free air anomaly and bias corrected airborne free air anomaly for varying filter parameters.

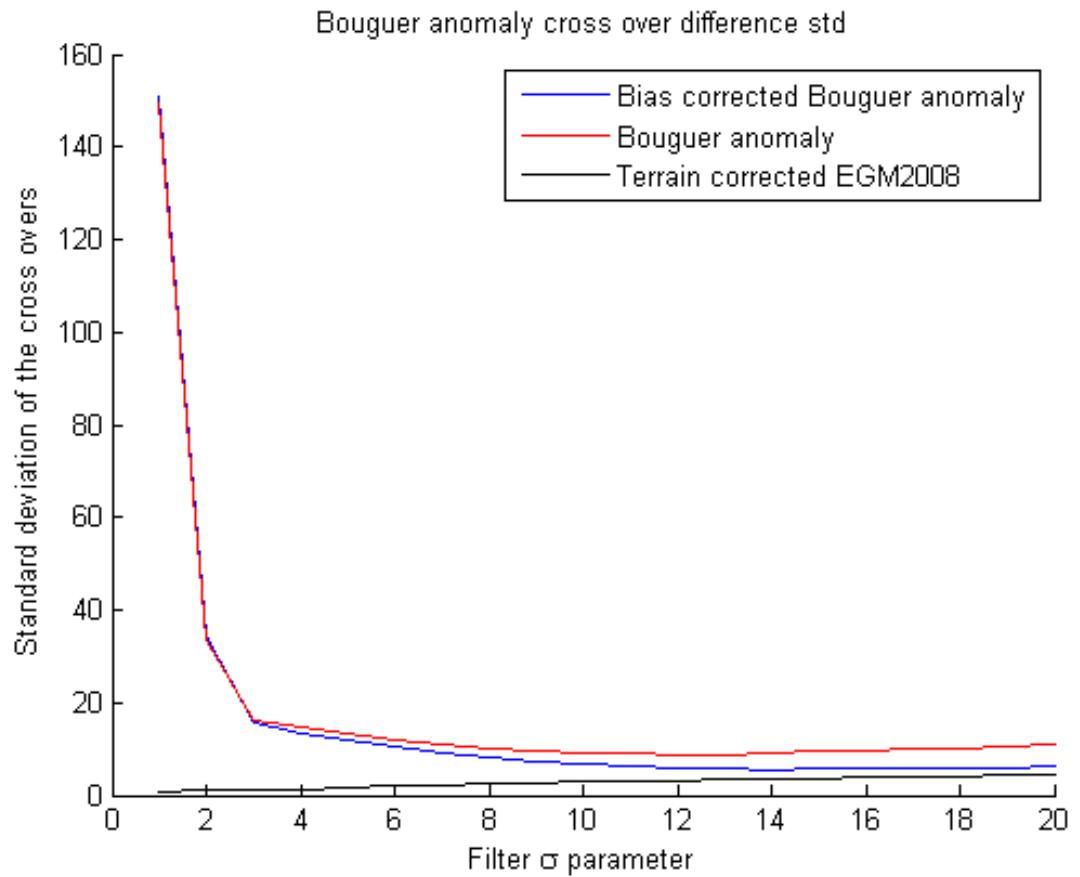

Figure 6.39: Standard deviation of the EGM2008, airborne Bouguer anomaly and bias corrected airborne Bouguer anomaly for varying filter parameters. 


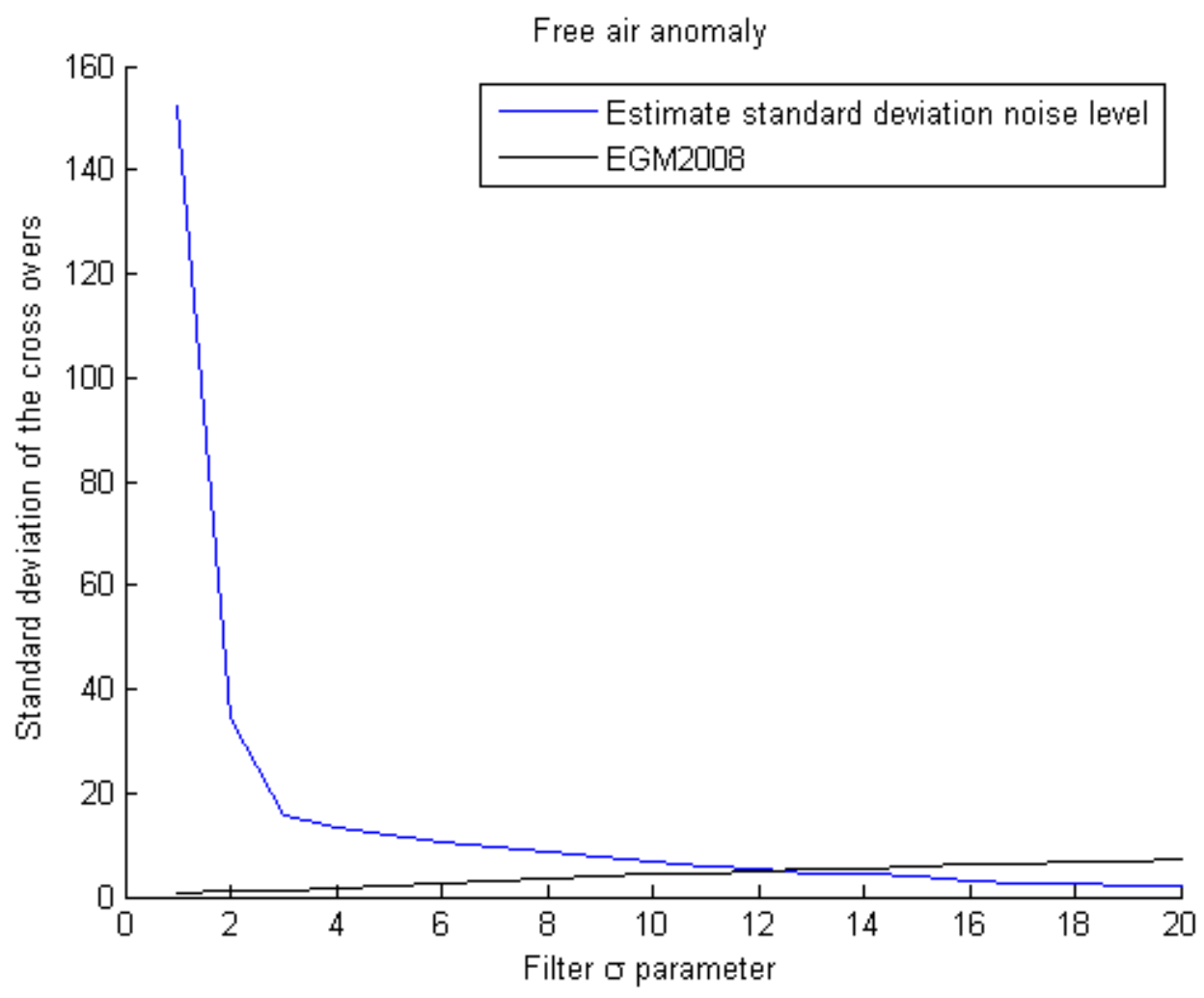

Figure 6.40: Standard deviation of the estimated noise level and cross-over difference standard deviation for EGM2008. The two lines intersect at around 120 seconds, this indicates that more gravity signal is being filtered than noise beyond 120 seconds.

The cross-over differences for the terrain corrected airborne gravity anomalies are substantially smaller than those for the free air gravity anomalies.

The along track bias adjustment improved the cross-over agreement for the free air and Bouguer anomaly data and the cross-over error for large filter lengths agrees better with EGM2008 than without the bias adjustment.

It can be seen that a filter parameter of around 120 seconds is optimal. This is because it produces the smallest cross-over difference in the free air anomaly and Bouguer anomaly data sets and the estimated signal error is equal to the estimated signal attenuation as seen in figure 6.40 .

With an along track filter parameter of 120 seconds, the cross-over differences of the bias corrected airborne Bouguer anomaly data have a standard deviation of $5.9 \mathrm{mGal}$. 


\section{Cross-over error after continuing the flight line data to the height of the cross lines using least squares collocation.}

To account for the variable height in the flight line data when estimating the cross-over error, the gravity anomalies from the flight lines which are not labelled as cross tie lines were upward/downward continued on to the flight lines which are labelled as cross ties. Here the filter parameter was chosen to be 120 seconds which is consistent with previous analysis. The continuation was done using least squares collocation as follows,

1. $i$ residual gravity anomalies $\Delta g_{i}=\Delta g\left(x_{i}, y_{i}, z_{i}\right)$ were obtained for the flight lines, which were not cross ties, and $k$ residual gravity anomalies $\Delta g_{k}=\Delta g\left(x_{k}, y_{k}, z_{k}\right)$ were obtained for the flight lines, which were cross ties, following steps $1-4$ of section 6.5 .

2. A matrix $C_{g s}$ was determined such that for each of the intersection points, $\left(x_{j}, y_{j}, z_{j}\right)$, along the cross tie lines,

$$
\left(C_{g s}\right)_{i, j}=-f \sum_{k=0}^{k=3} \alpha_{k} \log \left(D_{k}+\sqrt{\left(x_{i}-x_{j}\right)^{2}+\left(y_{i}-y_{j}\right)^{2}+\left(D_{k}+z_{i}+z_{j}\right)^{2}}\right)
$$

where $C_{0}=75.8 \mathrm{mGal}, D=9.5 \mathrm{~km}$ and $T=48.5 \mathrm{~km}$ and $f$ is calculated using equation 6.26 as determined previously.

3. a similar matrix $C_{g g}$ was obtain for the $i$ residual gravity anomalies $\Delta g_{i}=\Delta g\left(x_{i}, y_{i}, z_{i}\right)$ by equation 6.27 .

4. a residual gravity signal $\hat{\Delta g_{j}}$ was obtained at the intersection points from the $\Delta g_{i}$ 's as follows

$$
\hat{\Delta g_{j}}=C_{g s}\left(C_{g g}+N\right)^{-1} \Delta g_{i}
$$

$\mathrm{N}$ is of the same form as for equation 6.29.

This operation has been performed for the Bouguer anomaly airborne data both before and after the bias correction. The $\hat{\Delta} g_{j}$ values have then been compared to the $\Delta g_{k}$ values at the intersection points and the empirical cdf can be seen in figure 6.41 .

The standard deviation of the cross-over differences before the bias adjustment has been reduced from $8.4 \mathrm{mGal}$ to $7.9 \mathrm{mGal}$ and after the bias adjustment from $6.3 \mathrm{mGal}$ to $5.9 \mathrm{mGal}$ by the least squares collocation continuation. The standard deviations have been fitted to the inner $68 \%$ of the distributions to avoid the influence of outliers.

The improvement in the cross-over error before and after the upward continuation indicates that the varying flight line elevations impact on the cross-over error. Again here, the cross-over for the bias adjusted data set is smaller than the flight lines without the bias adjustment. 


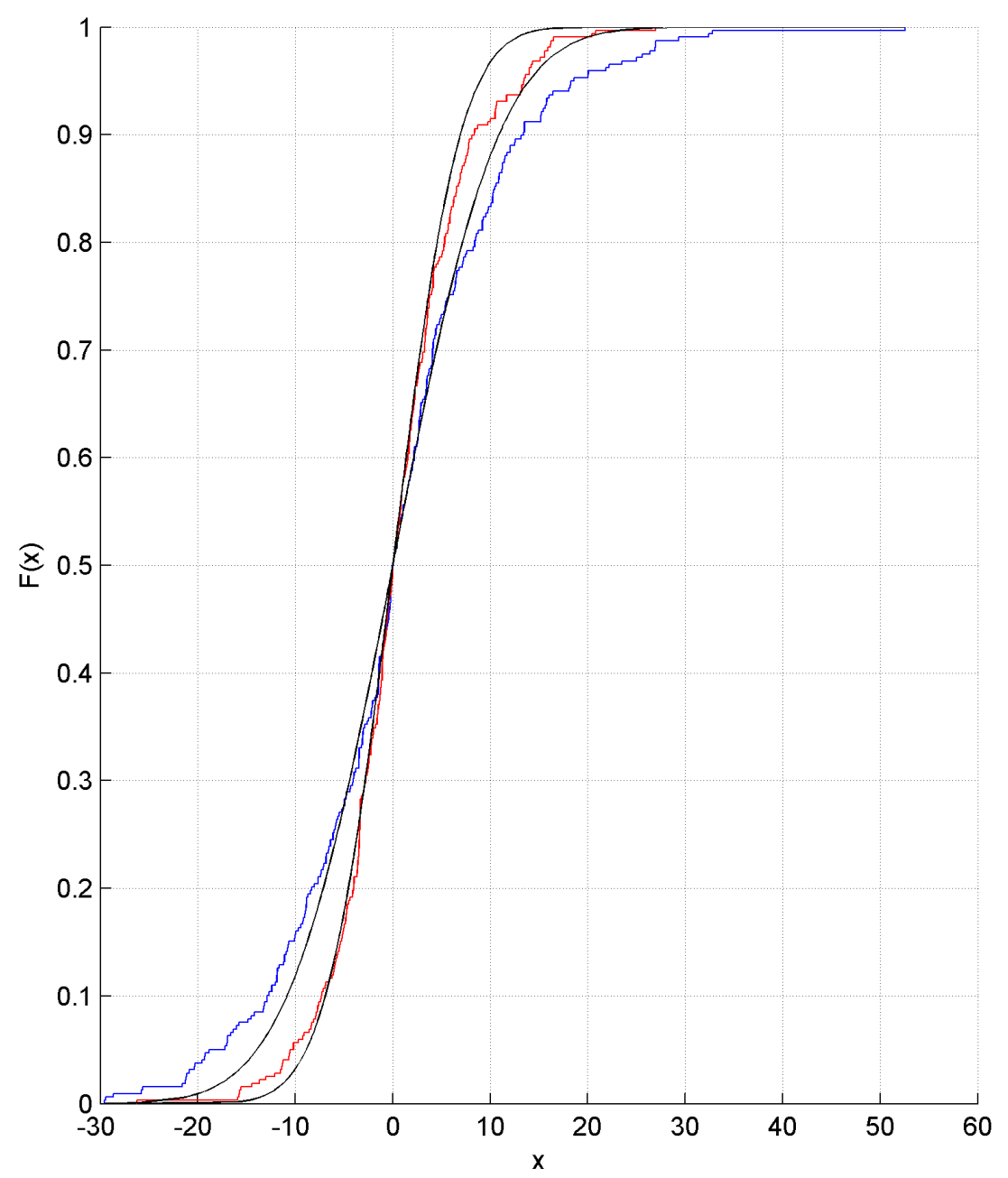

Figure 6.41: Empirical CDF plot of cross-over differences of least squares collocation downward continued airborne Bouguer gravity anomaly to the cross tie measurement locations. Before bias adjustment is plotted in blue and after the bias adjustment in red. Gaussian distributions are fitted to the inner $68 \%$ of each empirical distribution and are plotted in black. The standard deviation of the (inner $68 \%$ of) cross-over differences before the bias adjustment is $7.9 \mathrm{mGal}$ and after the bias adjustment is $5.9 \mathrm{mGal}$.

\subsubsection{Comparison to the terrestrial gravity data.}

The airborne gravity measurements have been downward continued by least squares collocation to the height of the topographic surface (estimated from the $8 \mathrm{~m}$ digital elevation model used for the terrain corrections) and estimated at the measurement locations. This calculation was performed as follows. 
1. Firstly, residual Bouguer gravity anomalies $\Delta g_{i}=\Delta g\left(x_{i}, y_{i}, z_{i}\right)$ were obtained for each observation $i=1,2, \ldots K$ in the flight line data. This was done by calculating the gravity anomaly at the locations of the flight line data from a terrain corrected GO_CONS_GCF _2_DIR_R5 global gravity model. The long wavelength model was used since rather than EGM2008 (as in section 6.5) since GO_CONS_GCF _2_DIR_R5 is a satellite only model (i.e. independent of the terrestrial gravity data) which ensures the downward continued airborne gravity data is independent from the terrestrial data for the comparison.

2. The GO_CONS_GCF_2_DIR_R5 gravity model was corrected for long wavelength terrain (by filtering the DEM to a $77 \mathrm{~km}$ wavelength following step 3 of section 6.5 ) this can be seen in figures $6.42 \mathrm{a}$ and $6.42 \mathrm{~b}$ of the GO_CONS_GCF_2_DIR_R5 gravity anomaly and GO_CONS_GCF_2_DIR_R5 corrected for $77 \mathrm{~km}$ wavelength terrain at the topographic surface.

3. The empirical covariance of the residual gravity anomalies (i.e. the airborne data minus the terrain corrected GO_CONS_GCF_2_DIR_R5 gravity anomaly data) was estimated and a logarithmic covariance function of the form of equation was fitted. The optimal parameters of the covariance function fitted to the empirical data were $C_{0}=576.42 \mathrm{mGal}$, $D=23.5 \mathrm{~km}$ and $T=46.5 \mathrm{~km}$ which were determined by the methodology of step 5 of section 6.5

4. The residual gravity signal $\hat{\Delta g_{l}}$ was then obtained at the terrestrial gravity locations $l=1,2,3, \ldots, L$ by solving the following equation

$$
\hat{\Delta g_{l}}=C_{g s}\left(C_{g g}+N\right)^{-1} \Delta g_{i} .
$$

Here, the matrix $C_{g g}$ was constructed such that elements in the matrix were given by

$$
\left(C_{g g}\right)_{i, j}=-f \sum_{k=0}^{k=3} \alpha_{k} \log \left(D_{k}+\sqrt{\left(x_{i}-x_{j}\right)^{2}+\left(y_{i}-y_{j}\right)^{2}+\left(D_{k}+z_{i}+z_{j}\right)^{2}}\right) .
$$

for every possible pair of residual gravity anomalies in the airborne data $\Delta g_{i}=\Delta g\left(x_{i}, y_{i}, z_{i}\right)$, and $\Delta g_{j}=\Delta g\left(x_{j}, y_{j}, z_{j}\right) i=1,2,3, \ldots, K$ and $j=1,2,3, \ldots, K$, i.e. $C_{g g}$ is a $K \times K$ variance covariance matrix of the data.

The matrix $C_{g s}$ is a $K \times K$ matrix of covariance values calculated such that for each pair of airborne observations $\Delta g_{i}$ and terrestrial gravity observations, $\Delta g_{l}$,

$$
\left(C_{g s}\right)_{i, l}=-f \sum_{k=0}^{k=3} \alpha_{k} \log \left(D_{k}+\sqrt{\left(x_{i}-x_{l}\right)^{2}+\left(y_{i}-y_{l}\right)^{2}+\left(D_{k}+z_{i}+z_{l}\right)^{2}}\right)
$$

where $\mathrm{N}$ is a $K \times K$ diagonal matrix with values on the diagonal corresponding to the variance of the airborne gravity data uncorrelated noise here set to $9 \mathrm{mGal}^{2}$.

5. The long wavelength terrain corrected GO_CONS_GCF_2_DIR_R5 gravity model was then restored to the residual gravity anomalies $\hat{\Delta g_{l}}$ to obtain the Bouguer anomaly which can then be compared to the terrestrial gravity observations.

The difference between the terrestrial and airborne Bouguer anomalies before and after the bias is shown in figures $6.43 \mathrm{a}$ and $6.43 \mathrm{~b}$. Figure 6.44 shows the empirical cdf plots of the differences / best fitting Gaussian distributions and table 6.5 gives the root mean squared, standard deviation of the inner $68 \%$, mean, min, max and kurtosis statistics of the differences. 

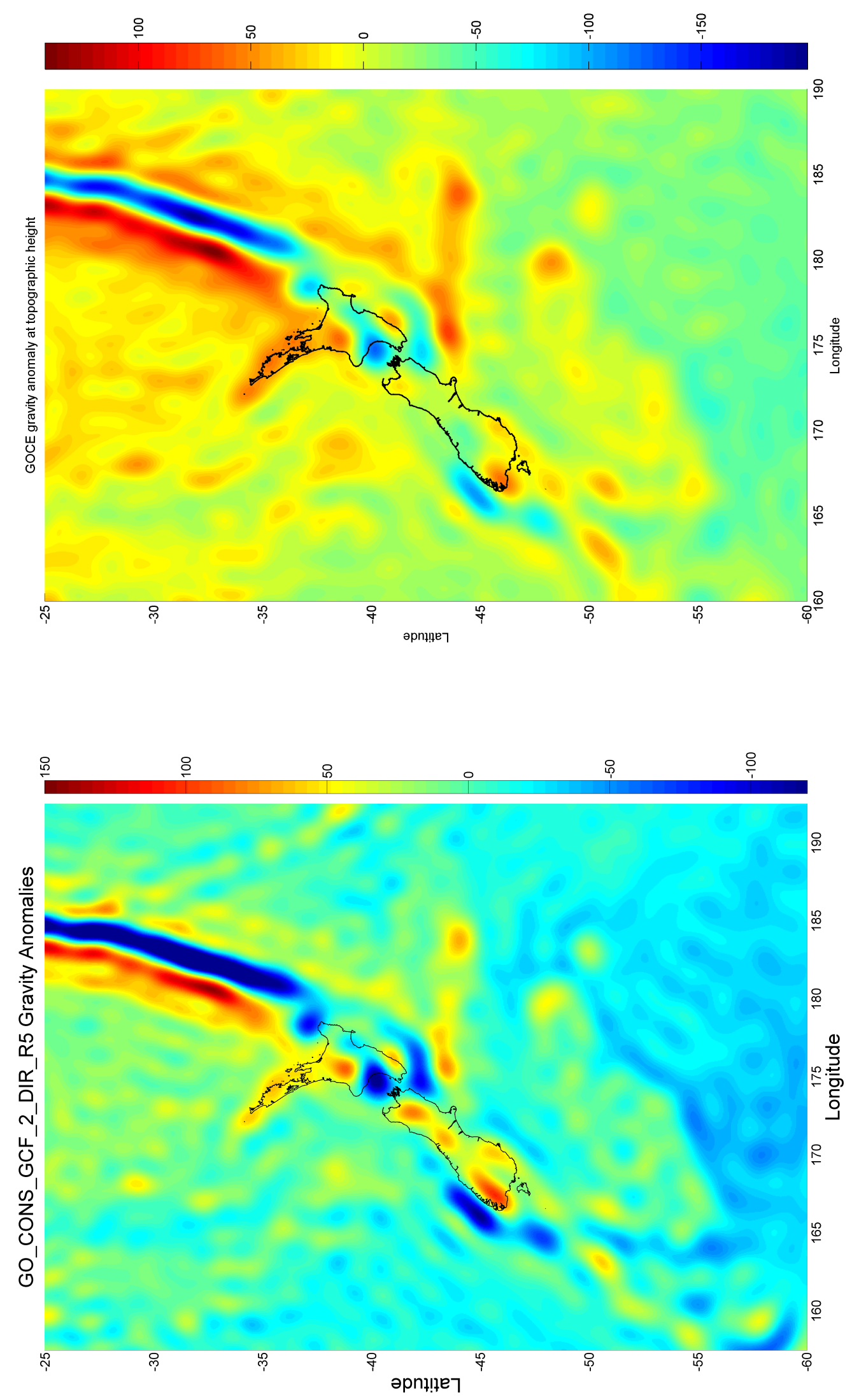

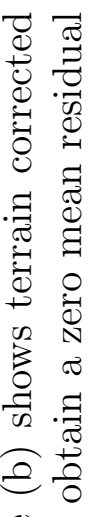

요

e

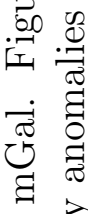

$\exists$.

궁

क्ष.

ํ.

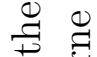

फ

ㅂ.

엉

Ð

$\approx$

궁

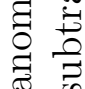

용

管

50

g.

驺

E

के

ฮ)

:

50

$\ddot{\Upsilon}$ ㅎํㅇ

ํํ สี สี

㝴

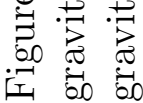



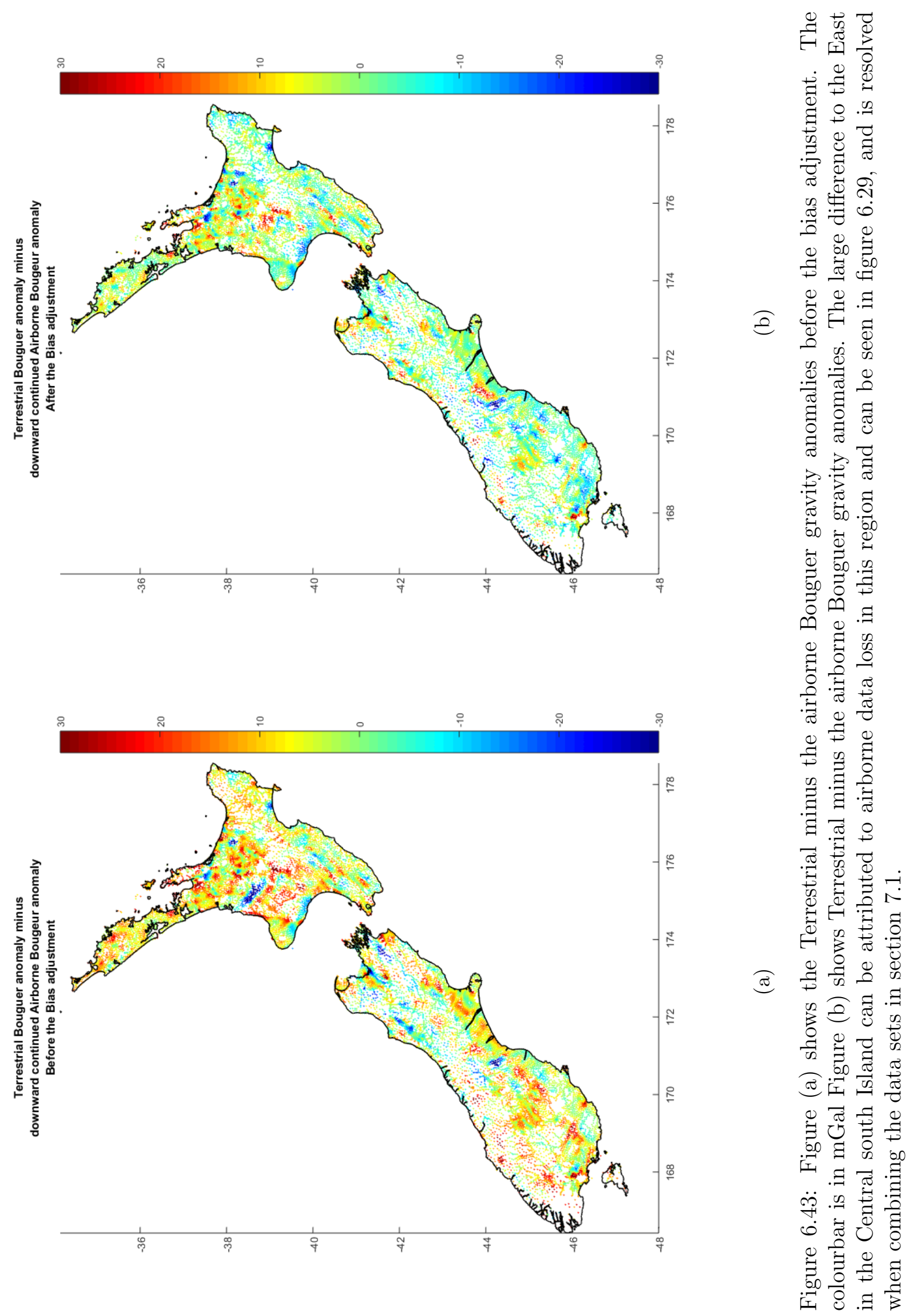
Table 6.5: Statistics of the Terrestrial Bouguer anomaly minus the downward continued airborne gravity data before and after the bias correction. The airborne data after the bias adjustment fit the terrestrial gravity data better.

$\begin{array}{lllllll} & \text { RMS } & \text { std } & \text { mean } & \text { min } & \text { max } & \text { kurtosis } \\ \text { Before bias adjustment } & 10.4750 & 7.8671 & 5.8688 & -95.1232 & 77.7873 & 5.1820 \\ \text { After bias adjustment } & 7.7943 & 7.0517 & 1.0444 & -100.7074 & 77.9590 & 5.2952\end{array}$

It can be seen here that the bias adjusted downward continued airborne gravity anomaly has a significantly better agreement with the terrestrial gravity data. This is further evidenced in the empirical cdf plots below.

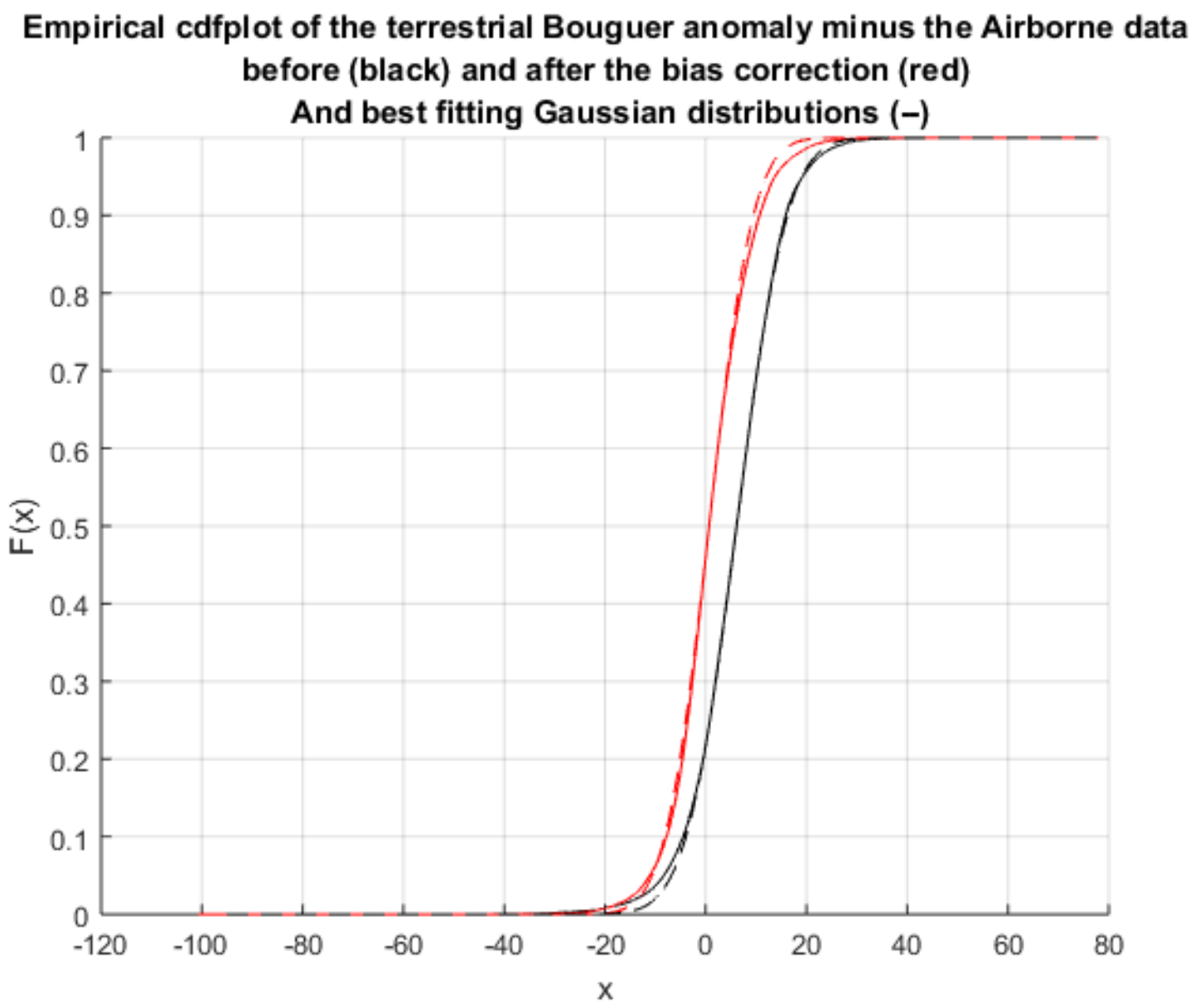

Figure 6.44: Empirical cdf plots of the terrestrial Bouguer anomaly data minus the downward continued airborne data before (black) and after the bias correction (red). Gaussian distributions fitted to the inner $68 \%$ of the distributions are seen as dashes. 


\subsubsection{Repeatability of the airborne gravity data - the calibra- tion lines}

The repeatability of the survey data was tested by flying over two calibration flight lines multiple times. One calibration line is on the North Island directly above High Level Road near Taupo and the second is located in Canterbury on the South Island. Both of the two flight lines are about $60 \mathrm{~km}$ long and are marked in red on figure 6.1 .

Five successful flights were made along the North Island flight line. The five lines of data along the calibration line do not contain measurements at exactly the same locations, for this reason an "average" track was estimated from the five flights. The recorded free air anomalies along the five single tracks where then interpolated to the average track using a nearest neighbour procedure. Figure 6.45 illustrates the (bias corrected) free air anomaly of the five separate flights along the North Island calibration line and an average in green.

The standard deviation from the average was calculated by generating a 1000 random sample points along the five lines. Their distribution from the mean free air anomaly along the line was then assessed to have a standard deviation of $2.21 \mathrm{mGal}$.

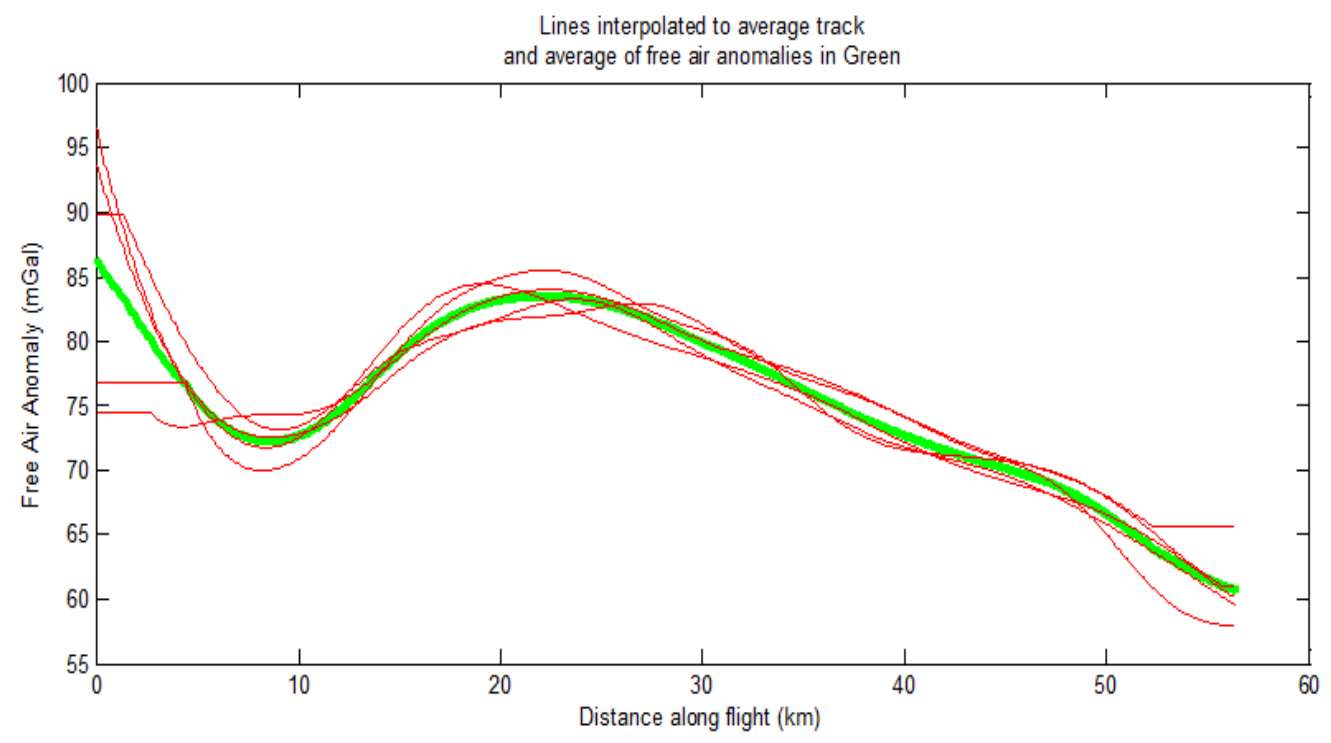

Figure 6.45: The free air anomaly of 5 separate flights, corrected for flight line biases, along the North Island calibration line and an average in green. The standard deviation from the average is $2.21 \mathrm{mGal}$.

Similarly, Five successful flights were made along the South Island flight line. Again, an "average" track was estimated from the five flights and the recorded free air anomalies along the five single tracks were then interpolated to the average track using a nearest neighbor procedure. Figure 6.46 illustrates the free air anomaly of the five separate flights along the South Island calibration line and an average in green. The standard deviation from the average was calculated the same as was for the North Island calibration line and has a standard deviation of 2.53 
mGal.

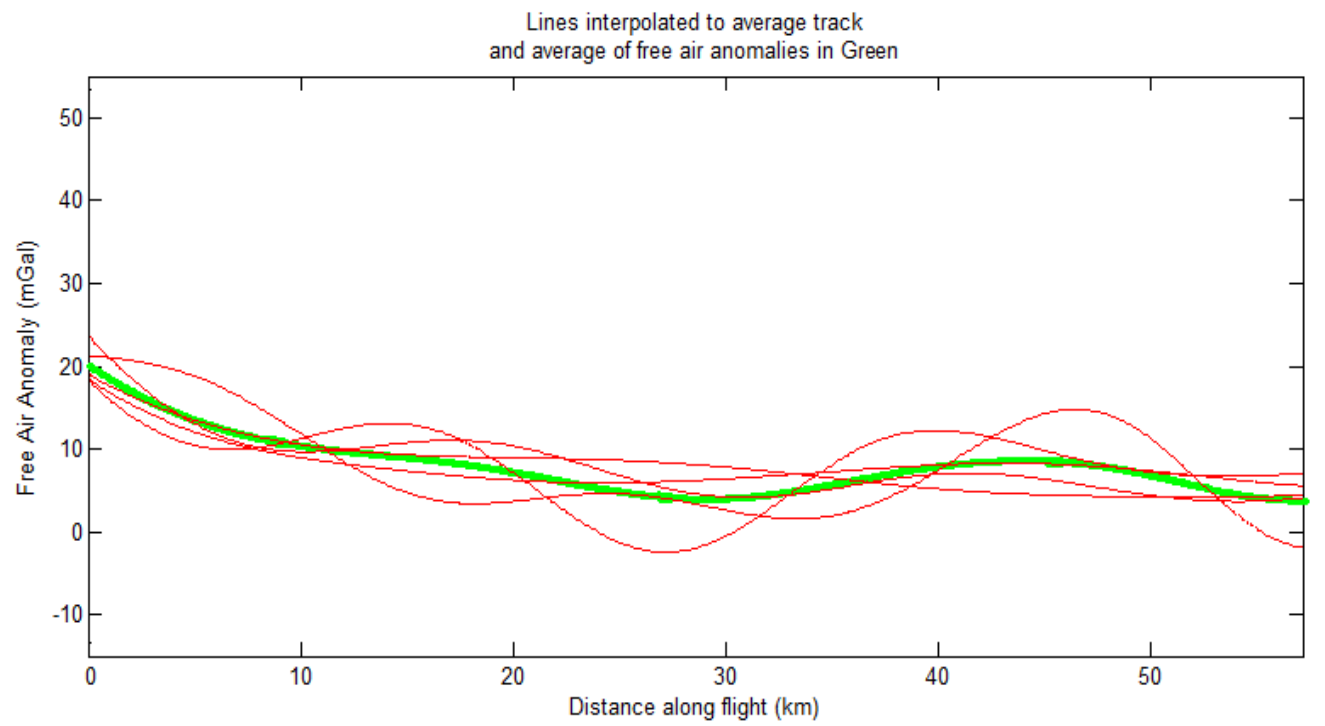

Figure 6.46: The free air anomaly of 5 separate flights along the South Island calibration line and an average in green. The standard deviation from the average is $2.53 \mathrm{mGal}$.

Terrestrial gravity observations were made along both calibration lines.

For the North Island, the locations of the terrestrial gravity observations were determined using a hand held GPS device. More specifically, a Garmin eTrex receiver, which is a recreational grade GPS (only) receiver. This type of hand held receiver provides an autonomous position and the expected accuracy of this device is $\pm 15 \mathrm{~m}$ horizontally and $\pm 50 \mathrm{~m}$ vertically. Moreover, the observation environment was generally poor due to the mature forestry obstructing sky visibility to both the East and West at all times. This resulted in ellipsoid height estimates which were only accurate to around 50 metres. For this reason the heights were estimated from the 8 DEM which should be accurate to approximately $10 \mathrm{~m}$. In terms of the free air and Bouguer slab corrections this translates to approximately $3 \mathrm{mGal}$ accuracy.

For the South Island, coordinates were obtained using a Trimble R6 which is a survey grade GNSS (Global Navigation Satellite System) antenna/receiver. This model observes both GPS and GLONASS satellite systems. The base station was provided via the Trimble iBASE, Virtual Reference Station (VRS) network. Observations, of 2 minute epochs, were made using RTK (real-time kinematic) with network solutions provided in real time via a mobile link to the VRS network. The observation environment was good although occasionally sky visibility was restricted by shelterbelts to the south. These measurements are accurate to $2 \mathrm{~cm}$ horizontally and $4 \mathrm{~cm}$ vertically. In terms of the free air and Bouguer slab corrections this translates to approximately $0.1 \mathrm{mGal}$ accuracy.

Terrain corrections for the terrestrial gravity observations along the calibrations lines were computed from the 8 metre digital elevation model out to $120 \mathrm{~km}$ to reduce the effect of topographical aliasing when comparing the airborne and terrestrial measurements. The topography corrected airborne gravity data were downward continued to the topographic surface along the calibration lines by least squares collocation as discussed in section 6.6.2. 
Figures 6.47 and 6.48 show the terrestrial Bouguer gravity anomalies in blue and downward continued Bouguer gravity anomalies in red for the North and South Island calibration lines plotted west to east. The statistics of the differences are given in table 6.6.

\section{North Island calibration line downward continued airborne data in red Terrestrial observations in blue}

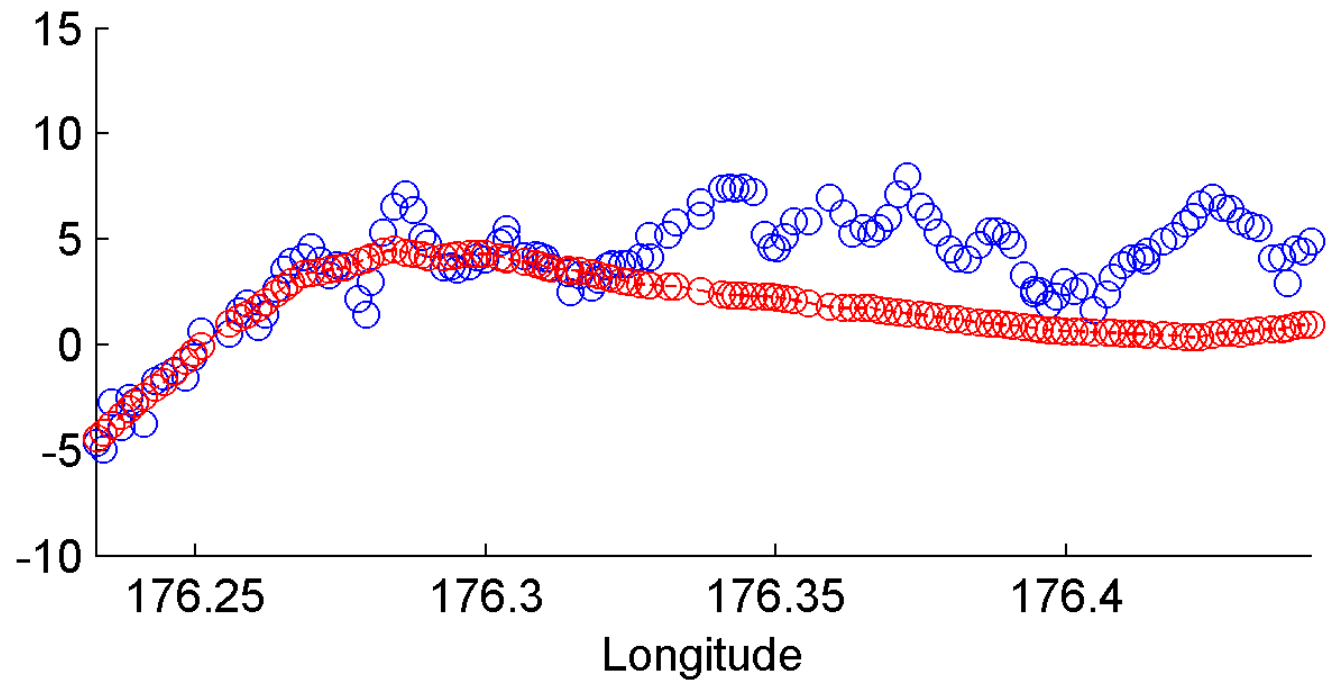

Figure 6.47: The airborne Bouguer anomaly downward continued to the topographic surface along the North Island calibration line in red and terrestrial Bouguer anomaly in blue. 


\section{South Island calibration line downward continued airborne data in red Terrestrial observations in blue}

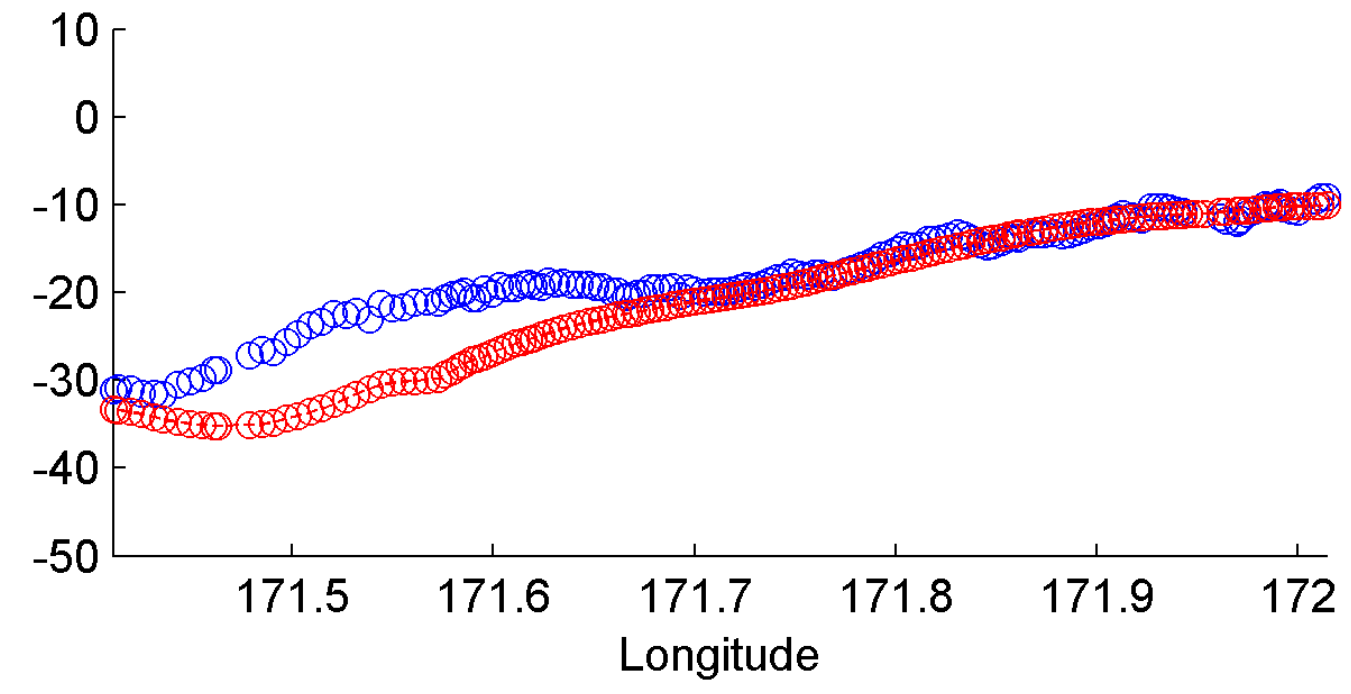

Figure 6.48: The airborne Bouguer anomaly downward continued to the topographic surface along the South Island calibration line in red and terrestrial Bouguer anomaly in blue.

Table 6.6: Statistics of the terrestrial Bouguer gravity anomalies minus the downward continued Bouguer gravity anomalies for the North and South Island calibration lines.

$\begin{array}{lllll} & \text { RMS } & \text { std } & \text { min } & \text { max } \\ \text { North Island } & 2.98 & 2.13 & -2.63 & 6.51 \\ \text { South Island } & 4.18 & 3.28 & -1.36 & 10.22\end{array}$

\subsubsection{Summary of accuracy assessment}

The accuracy of the airborne gravity data has been assessed by three methods.

1. The cross-over error, this is the difference between observed gravity values at flight line intersection points

After accounting for along track biases and varying flight line elevations the standard deviation of the cross-over differences was shown to be approximately $5.9 \mathrm{mGal}$.

2. A comparison to terrestrial gravity measurements. After accounting for along track biases the flight data were downward continued by least squares collocation and compared to the terrestrial gravity data. The difference between the two data sets had a standard deviation of $7.1 \mathrm{mGal}$.

3. Repeated flights along two flight lines, one on the North Island and one on the South Island demonstrated a repeatability of 2.2 and $2.5 \mathrm{mGal}$ respectively. Terrestrial gravity data were also obtained along these flight lines and had an agreement of $2.1 \mathrm{mGal}$ standard deviation for the North Island airborne data and $3.3 \mathrm{mGal}$ for the South Island.

The cross-over differences are largely influenced by the along track filter which improperly attenuates the gravity signal. This indicates that the cross-over error is perhaps not the best 
indication the airborne gravity data accuracy.

Section 4.5 demonstrated that the terrestrial gravity data base is contaminated by positioning errors which propagates through into the gravity signal due to the corrections necessary to obtain the Bouguer anomalies. For this reason these data are perhaps not ideal to determine the accuracy of the airborne data.

The most reliable accuracy assessment is the repeatability along the calibration lines and comparison to the ground terrestrial gravity observations made along the flight line. This is because the along track filter is applied in the same direction spatially, and the terrestrial gravity observations have not been obtained with accurate positioning and consistent instrumentation. Over all here, the standard deviation of the discrepancies in the airborne data is around $3 \mathrm{mGal}$. 


\subsection{Summary}

In this chapter the methodology and apparatus used for airborne gravimetry has been discussed. The basic processing techniques are given and attention is given to the high noise level present in the raw airborne gravity signal, this is attributed to the observations being made on a moving platform.

An airborne gravimetry survey was conducted over New Zealand and the details of the survey and instrumentation used were given. The flight lines were spaced $10 \mathrm{~km}$ apart and a Lacoste and Romberg model S gravimeter was used to collect the data. The steps taken to reduce the acquired data to Bouguer gravity anomalies has been discussed in detail. The airborne data offer an improvement over the existing sparse gravity data over New Zealand since they are uniform and seamlessly cover on shore and shallow coastal areas.

The accuracy of the data has been examined by comparing gravity values at the intersection point of flight lines, comparing the downward continued airborne data to existing terrestrial gravity data and by comparing data for repeat flights along the two calibration lines. The accuracy of the data estimated from repeated flight lines and reliable ground truth data has been evaluated to be around $3 \mathrm{mGal}$. 


\section{Chapter 7}

\section{Combining Data Sets using Least Squares Collocation (LSC)}

\subsection{Introduction}

This chapter discusses the method of least squares collocation and how it can be used to combine scattered gravity observations (of different accuracies) onto a regular grid. A discussion of the background and methodology of least squares collocation is given. Here, the fundamental assumptions of the underlying gravity observations are specified and the key equations which are used to estimate the gravity signal from the scattered observations are given. The gravity observations must have a zero mean and typically the errors are assumed to be independent.

With the appropriate choice of covariance function, least squares collocation can be used for downward continuation. This means observations that have been made at varying elevations, such as airborne and terrestrial gravity data, can be combined. Several covariance functions are identified and the logarithmic covariance function is shown to have this three dimensional property.

The terrestrial gravity observations were discussed in detail in section 4.1 and their estimated errors were given in section 4.5 and are used to determine the noise matrix $N$ used for the least squares collocation process. Here the error estimates are shown to be too small in some areas which causes the gridded data to become too constrained at individual points. This results in "bulls eye" features. A revised minimum accuracy estimate is given and the results of adjusting the estimated errors are demonstrated.

The use of least squares collocation to downward continue the along track airborne gravity observations, and griding at regular intervals, is discussed. The filtering of the airborne gravity observations causes the along track observation errors to be correlated. It is demonstrated how the effect of the filtering can be appropriately incorporated in to the least squares collocation formulas.

A grid of combined airborne and terrestrial gravity observations is presented and the effect of combining the data sets is discussed. The combined grid draws on the strengths of both data sets by merging the uniform (but generally lower accuracy) airborne data and highly accurate (but non-uniform and in some areas sparse spatially) terrestrial data. This is demonstrated by looking at a grid of propagated errors. 
Two new data sets are introduced, the shipborne gravity data which were cross-over adjusted by Amos et al (2005) and a highly accurate satellite gravity anomaly dataset from Sandwell et al. (2014). Steps have been taken to remove potentially erroneous data on-shore from the satellite gravity data, and the accompanying error estimates of the satellite model are used to heavily down weight the influence of gravity values of the satellite gravity model in shallow coastal areas on the least squares collocation grid.

A final grid of gravity anomalies is presented which has been estimated from all available gravity observation sources. The observations are optimally combined by the appropriate choice of covariance function and a careful consideration of prior error estimates.

\subsection{Basic mathematical setting}

The methodology here is given Moritz (1972) but the text largely follows Reilly (1979). Least squares collocation (LSC) gives a method to interpolate gravity anomalies at a desired location from scattered observations. The desired signal is denote here as $\hat{s}=s(\hat{\theta}, \hat{\phi}, \hat{H})$ and the scattered gravity anomalies are denoted $\Delta g=\Delta g(\theta, \phi, H)$. Reilly (1979) says a $q \times 1$ vector of observed gravity anomalies $\Delta g$ can be written as follows,

$$
\Delta g=A x+s+n
$$

where $x$ is an $m \times 1$ vector of parameters $\mathrm{A}$ is a $q \times m$ matrix of coordinates, $s$ is a random (but spatially correlated) $q \times 1$ signal vector and $n$ is additive white noise. It is convenient to re-write equation 7.1 as,

$$
h=s+n=\Delta g-A x
$$

so that $h$ corresponds to the purely random part of the observed gravity signal. In practice $A x$ embodies any regular part of the gravity signal such as long wavelength gravity anomalies (estimated from a global gravity model) or in the case of section 6.5, the flight line biases. It is assumed that the signal $s$ and noise $n$ are uncorrelated and purely random, such that their expected value $M$ at each observation location is zero i.e.

$$
\begin{gathered}
C_{s n}=\operatorname{Cov}(s, n)=0, \\
C_{n, s}=\operatorname{Cov}(n, s)=0 .
\end{gathered}
$$

and

$$
M(s)=M(n)=0
$$

The covariance of the "observed" signal $h$ with itself can then be written as,

$$
C_{h, h}=C_{s, s}+C_{n, n}
$$

and the covariance of the "observed" signal $h$ and the desired signal $\hat{s}$ is given by $C_{\hat{s}, h}$. As a result of this the desired random signal $\hat{s}$ can be written as,

$$
\hat{s}=C_{\hat{s}, h} C_{h, h}^{-1} h .
$$

Equation 7.7 can then be used to interpolate the signal $s$ at any desired location. Under the assumption that the regular part of the gravity signal, which is subtracted from the observed 
gravity anomalies, is "known" the error covariance in the determined signal $E_{s, s}$ can be written as,

$$
E_{\hat{s}, \hat{s}}=C_{\hat{s}, \hat{s}}-C_{\hat{s}, h} C_{h, h}^{-1} C_{h, \hat{s}}
$$

where the diagonal elements of $E_{\hat{s}, \hat{s}}$ give the variances of the determined values $\hat{s}$ propagated from the error $n$ of the gravity observations.

In equation 7.7 there are $q$ observations, so the matrices $C_{s, s}$ and $C_{n, n}$ are of the size $q \times q$ and supposing that the signal $\hat{s}$ is desired at $p$ locations, $C_{\hat{s}, h}$ is a $p \times q$ matrix. The $C_{s, s}$ and $C_{n, n}$ matrix elements are determined from an analytical function known as a covariance function which are discussed in section 7.3.

The matrix $C_{n, n}$ is the variance covariance matrix of observation noise $n$, when the observations are assumed to be independent the matrix is strictly diagonal and a diagonal entry in row $i$ is the variance of observation $i$ which is given as a prior. Section 7.5 demonstrates that this is not necessarily appropriate for the along track filtered airborne gravity data and a method to estimate the off diagonal entries is given.

\subsection{Covariance functions}

In section 6.5 it was demonstrated how the empirical covariance of residual gravity observations (i.e. after subtracting a long wavelength gravity anomaly from a global gravity model) can be estimated for some airborne gravity data. The parameters of an analytical function are fitted to the empirical covariance values and then this function can be used to determine the matrix entries of $C_{s, s}$ and $C_{\hat{s}, h}$. The covariance matrix is positive definite and so this property is also required of the analytical function (called the covariance function). A wide range of functions have this property; some commonly used functions for 2D geophysical mapping are given in the following table.

Table 7.1: Commonly used covariance functions for geophysical mapping - adapted from Neda (2009).

$\begin{array}{llll}\text { Name } & \text { Covariance fun. } & \text { Parameter } & \text { Ref. } \\ \text { Exponential } & C_{0} e^{-\frac{r}{d}} & - & \text { Shaw et al. (1969) } \\ \text { Triangular } & C_{0}\left(1-\frac{r}{\alpha d}\right) & \alpha=2 & \text { Duquenne et al. (2005) } \\ \text { Gaussian } & C_{0} e^{-\alpha^{2} r^{2}} & \alpha=\frac{1}{d}(\log 2)^{1 / 2} & \text { Kearsley (1977) } \\ \text { Second order Markov } & C_{0}\left(1-\frac{r}{d}\right) e^{-\frac{r}{d}} & - & \text { Kasper (1971) }\end{array}$

In table 7.1, $r$ is the spatial separation between two gravity measurements in the (x,y) plane, $C_{0}$ is the variance of the signal $s$ and $d$ is the value of $r$ at which the covariance function value reduces to $\frac{C_{0}}{2}$. So the covariance between any two gravity observations can be determined from the covariance function by knowing the observation locations. For example, for two gravity anomalies in Euclidean space $\Delta g_{i}=\Delta g\left(x_{i}, y_{i}\right)$ and $\Delta g_{j}=\Delta g\left(x_{j}, y_{j}\right)$ the entry in row $i$ and column $j$ of matrix $C_{s, s}$ using the exponential covariance function can be written as,

$$
\left(C_{s, s}\right)_{i, j}=C_{0} e^{-\frac{r_{i, j} \log 2}{d}}=C_{0} e^{-\frac{\sqrt{\left(x_{i}-x_{j}\right)^{2}+\left(y_{i}-y_{j}\right)^{2}} \log 2}{d}}
$$


The Forsberg (1987) covariance function is 3 dimensional. The model gives the covariance between gravity anomalies in 3 dimensional Euclidean space, $\Delta g_{i}=\Delta g\left(x_{i}, y_{i}, H_{i}\right)$ and $\Delta g_{j}=$ $\Delta g\left(x_{j}, y_{j}, H_{j}\right)$, at two altitudes and takes the following form,

$$
\left(C_{s, s}\right)_{i, j}=-f \sum_{k=0}^{k=3} \alpha_{k} \log \left(D_{k}+\sqrt{r_{i . j}^{2}+\left(D_{k}+H_{i}+H_{j}\right)^{2}}\right)
$$

with

$$
f=C_{0} \log \left(\frac{(D+T)^{3}(D+3 T)}{D(D+2 T)^{3}}\right)
$$

again $r_{i, j}$ is the distance in the $x$ - $y$ plane between $\Delta g_{i}$ and $\Delta g_{j}, \alpha_{k}=1,-3,3,-1$ and $D_{k}=$ $D+k T$, where the parameters $D, T$ and $C_{0}$ are fitted to the empirical covariance values. Since this covariance function is 3 dimensional it can be used to downward continue airborne gravimetry data as is discussed in Forsberg (1995) and is used here for that purpose in sections 6.6 and 7.5 .

\subsection{General steps taken to grid gravity anomalies us- ing least squares collocation.}

In general, the procedure to grid/downward continue the gravity data using least squares collocation is broken down into the following steps.

1. Compute the Bouguer anomaly for each of the available gravity observations.

2. Calculate the long wavelength gravity anomaly at the location at each of the gravity observations from a topography corrected global gravity model. Here the satellite only GOCE direct 5 model has been used throughout.

3. Subtract the long wavelength GGM gravity signal from the Bouguer anomaly data to obtain residual gravity observations.

4. Calculate the empirical covariance of the residual gravity observations and find the best fitting analytic logarithmic covariance model parameters, $C_{0}, D$ and $T$.

5. Calculate the covariance matrix, $C_{s, s}$, of residual gravity observations, and the entries for the noise matrix $C_{n, n}$.

6. Calculate the desired signal $s$ by equation 7.7 and the propagated error of the residual gravity signal $E_{\hat{s}, \hat{s}}$ by equation 7.8 .

7. Restore (add back) the long wavelength gravity model.

These steps are explained in detail in section 6.5 when dealing with the airborne gravity data. As discussed in section 6.5, the vast amount of data causes the matrices $C_{s, s}, C_{n, n}$ and $C_{\hat{s}, h}$ to be impractically large. To resolve this issue, here, the least squares collocation has been performed in a series of 1 degree blocks with a 15 arc minute over lap between each. This reduces the matrices size to around $4000 \times 4000$ on average and there are no evident discontinuities between the blocks. 


\subsection{Combining all of the available datasets into a sin- gle gravity anomaly grid}

A grid of latitudes and longitudes has been established with a cell size of 1 arc minute over the range of $25^{\circ}(\mathrm{S})$ to $60^{\circ}(\mathrm{S})$ and from $160^{\circ}(\mathrm{E})$ to $190^{\circ}(\mathrm{E})$. The mean topographic height was then determined for each 1 arc minute cell by block averaging the $8 \mathrm{~m}$ DEM (described in sections 6.3.6 and 4.4), and database recorded heights where the DEM is not available. The averaged topographic map is shown in figure 7.1. The $(x, y, z)$ locations of this grid are used throughout this section as the points to where the gravity data are interpolated.

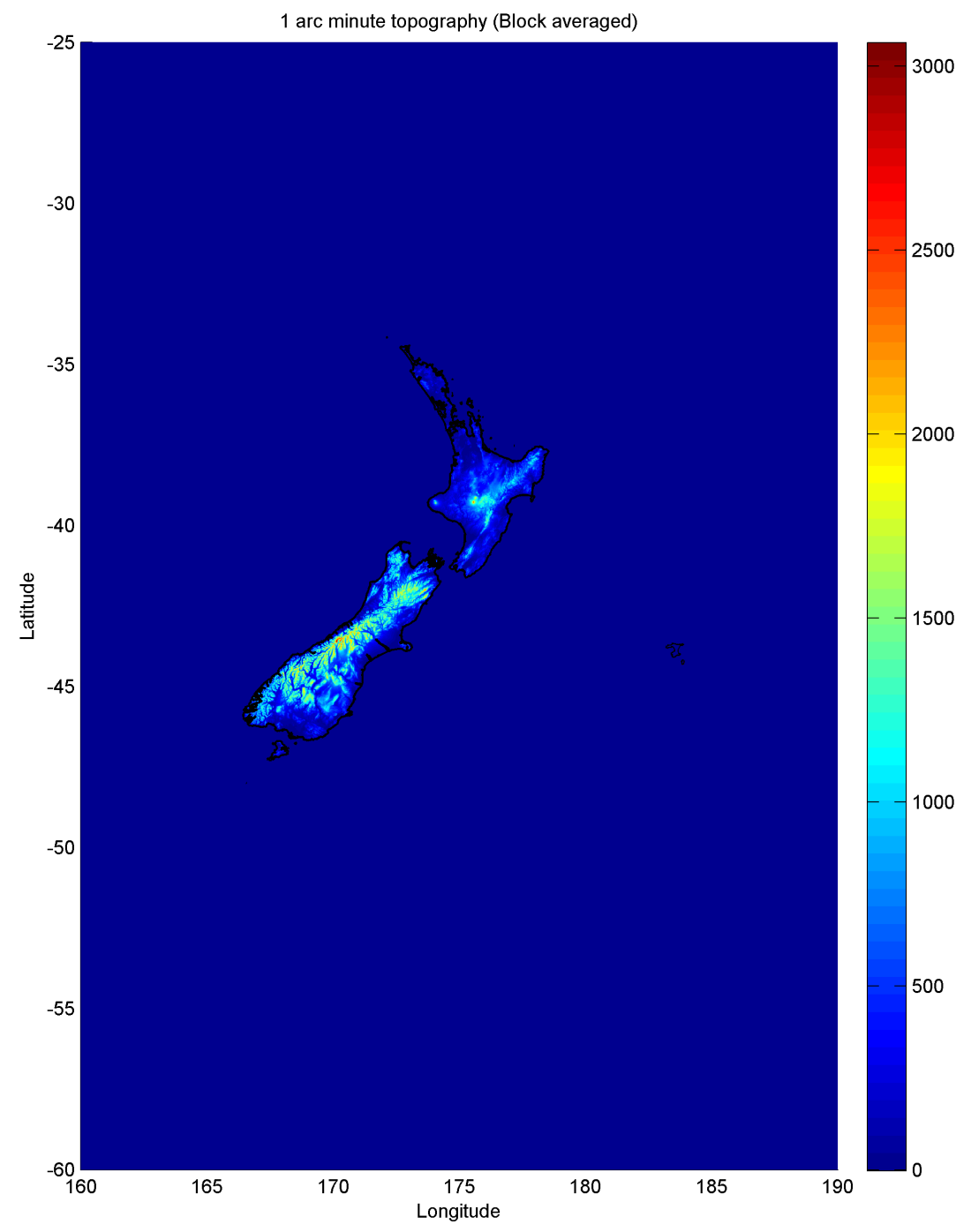

Figure 7.1: 1 arc minute grid of topographic heights from block averaged $8 \mathrm{~m}$ DEM heights. This figure shows the full computation area although in other figures throughout this section the area has been restricted for illustrative purposes. 
The Terrestrial gravity data: The terrestrial Bouguer gravity anomaly data of section 4.4 have been gridded using least squares collocation. Noise level standard deviations have been assigned to the observations in section 4.5 and have been squared and used as the diagonal elements of the matrix $N$ in the least squares collocation procedure. The resulting gravity anomaly grid and propagated error map are shown in figures 7.2 and 7.3.

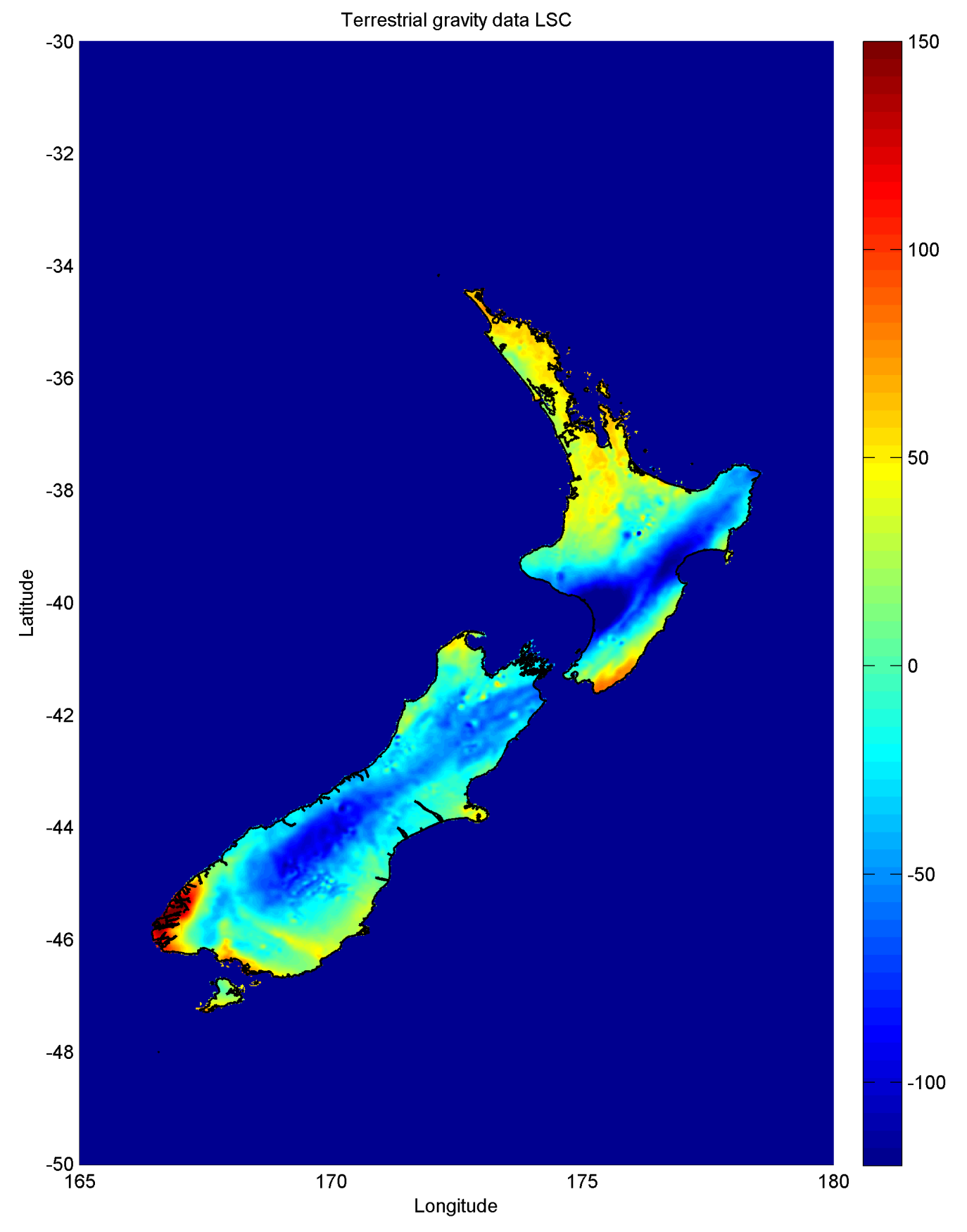

Figure 7.2: Least squares collocation gridded terrestrial gravity observations in mGal. 


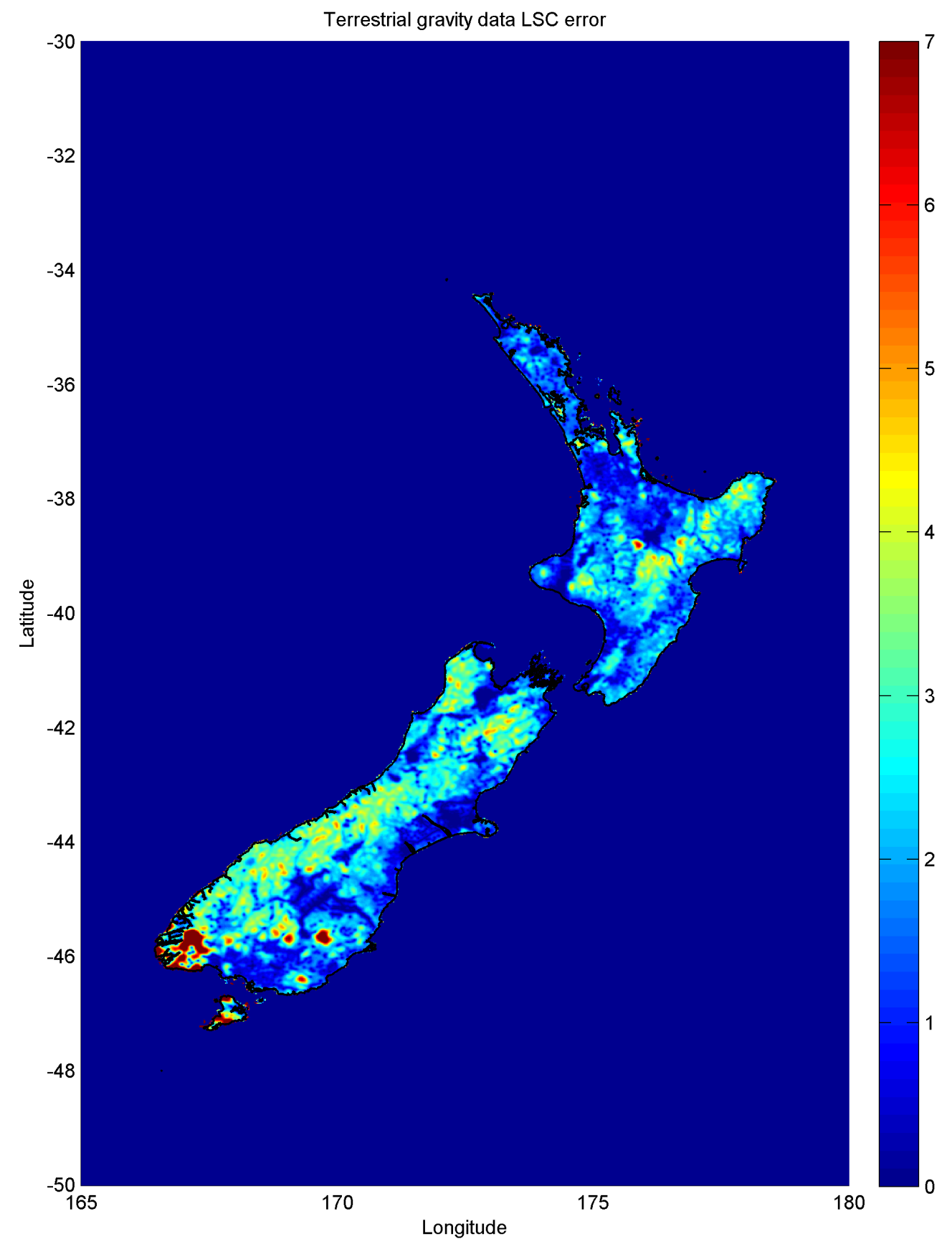

Figure 7.3: Least squares collocation gridded propagated terrestrial gravity observations error in mGal.

There are several "bulls eye" features in the gravity anomaly map which are undesirable. Most notable is one near the Taupo Volcanic Zone and a suite of small artifacts in Otago, these features are shown in figures $7.4 \mathrm{a}$ and $7.4 \mathrm{~b}$. 


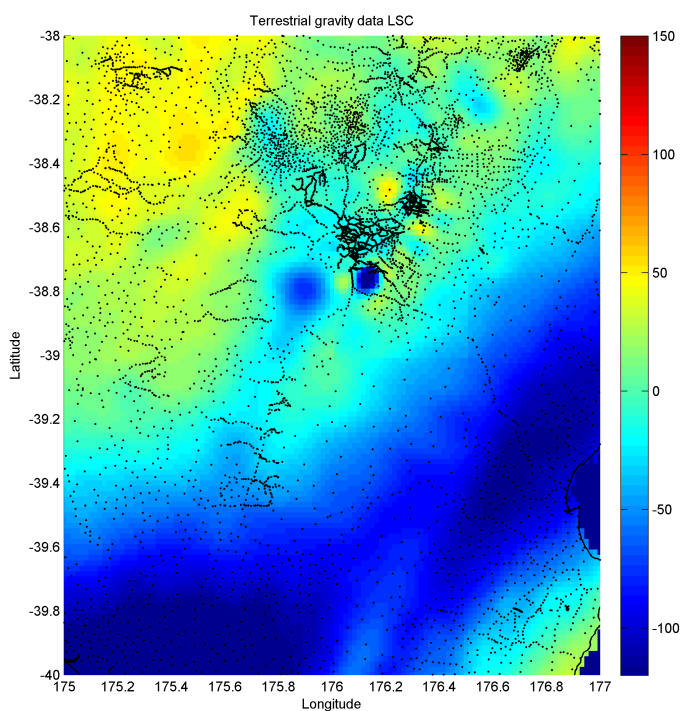

(a)

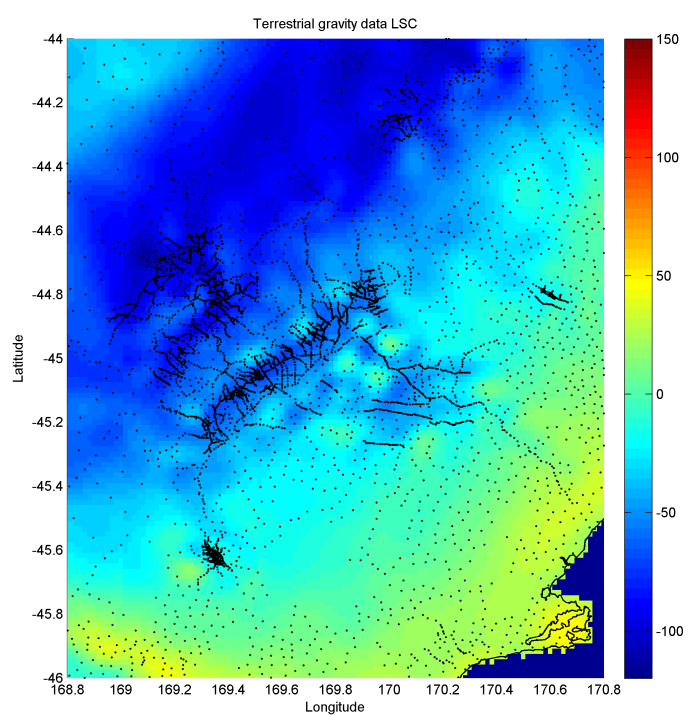

(b)

Figure 7.4: Least squares collocation gridded terrestrial gravity observations in mGal in the Taupo volcanic zone (a) and Otago region (b). There are undesirable "bulls eye" artifacts in the gridded data. The black marks indicate the measurement locations.

These kinds of artifacts generally seem to occur where there is a transition from dense data with particularly small noise levels to sparse areas of data coverage, as can be seen in figures $7.5 \mathrm{a}$ and $7.5 \mathrm{~b}$ showing the propagated error and data density in each region.

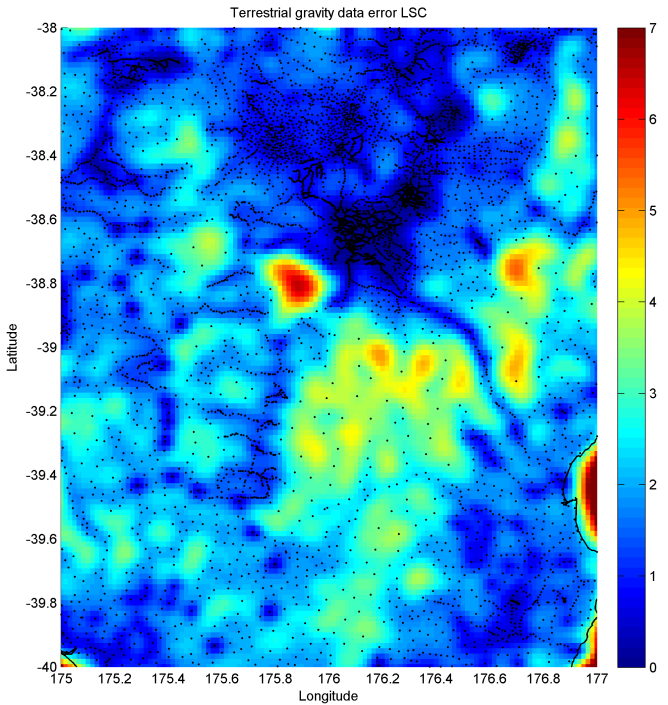

(a)

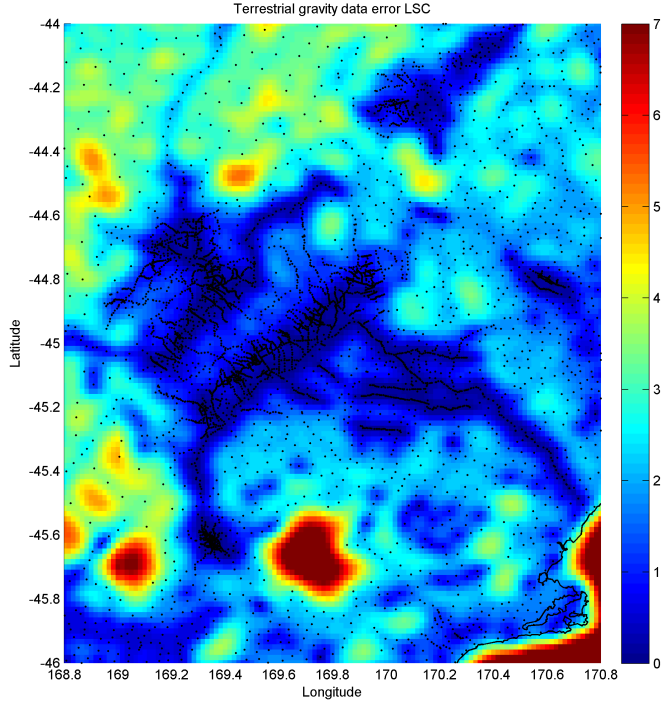

(b)

Figure 7.5: Least squares collocation gridded terrestrial gravity observations error in $\mathrm{mGal}$ in the Taupo volcanic zone (a) and Otago region (b).

To resolve this problem, the smallest noise level assigned to the data has been set to $1 \mathrm{mGal}$, which allows for more flexibility in the gridded data where data density and accuracy change. The resulting gravity anomaly and propagated error maps are shown in figures 7.6a and 7.6b. 

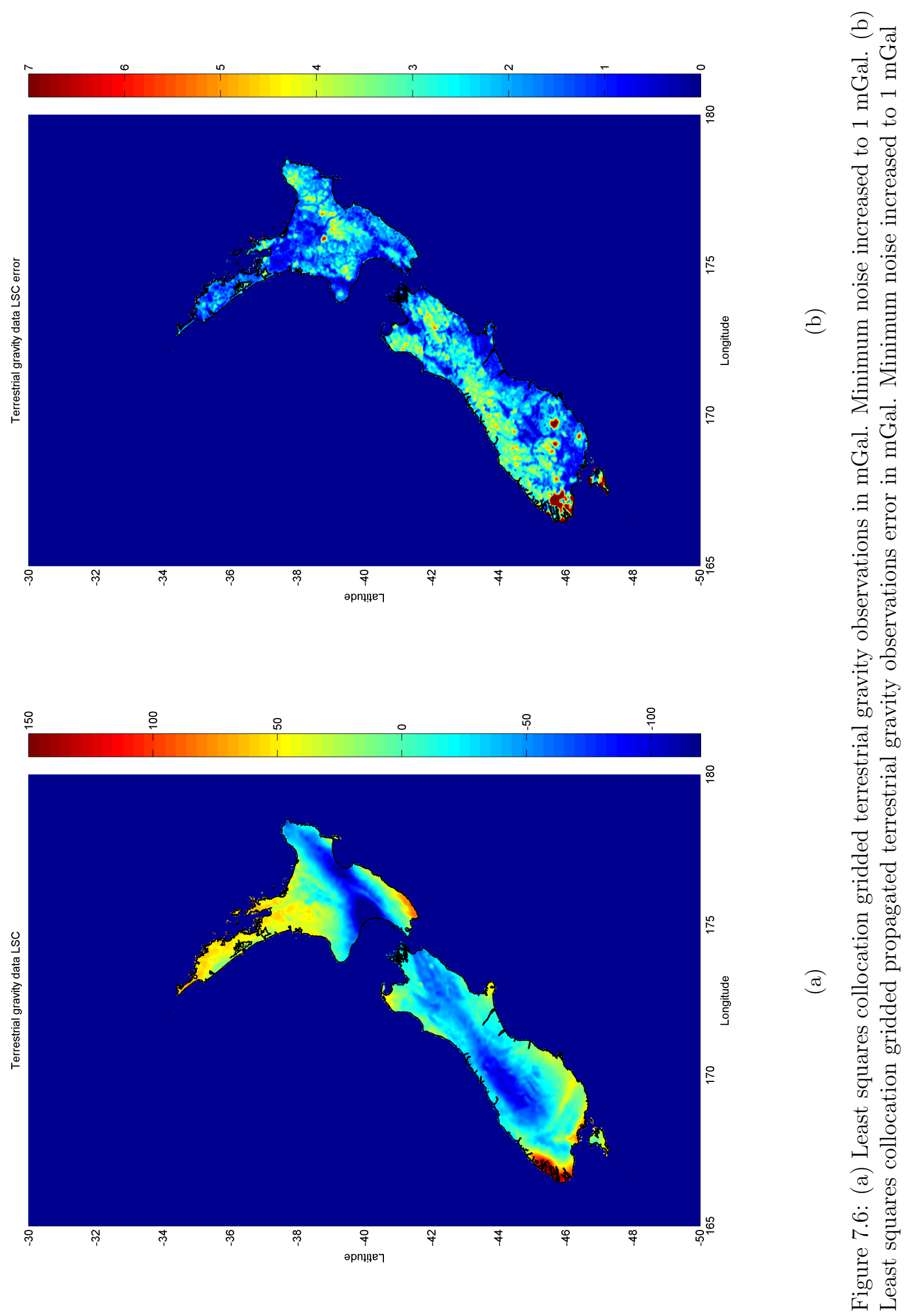
For the two test regions, the Taupo volcanic zone region and Otago, the anomalous artifacts are no longer evident in the gridded data as can be seen in figures $7.7 \mathrm{a}$ and $7.7 \mathrm{~b}$.

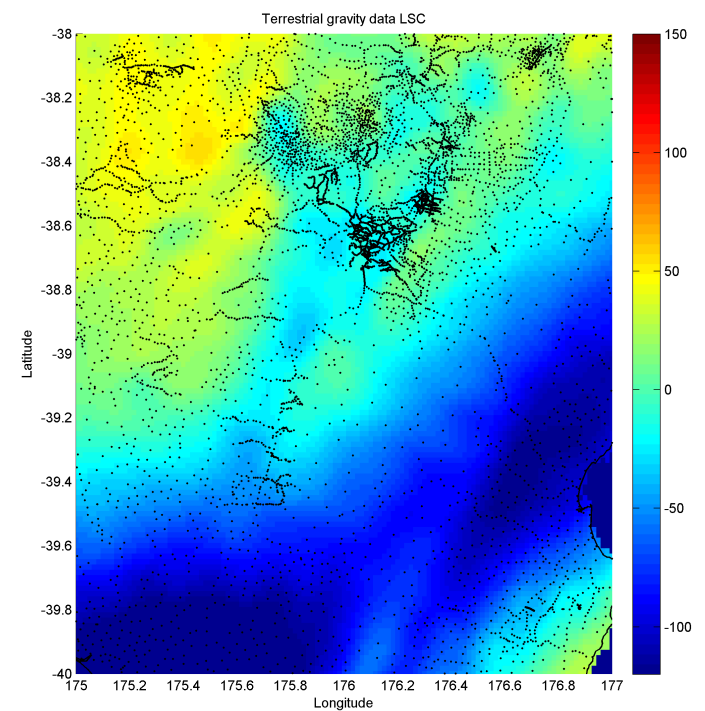

(a)

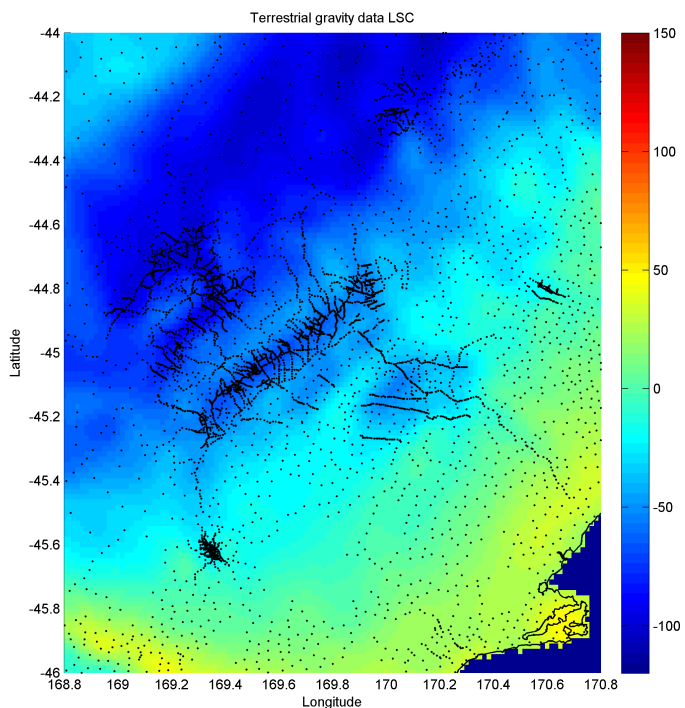

(b)

Figure 7.7: Least squares collocation gridded terrestrial gravity observations in mGal in the Taupo volcanic zone (a) and Otago region (b). The undesirable "bulls eye" artifacts in the gridded data have been removed by increasing the minimum noise level to $1 \mathrm{mGal}$.

Figure 7.8 shows the difference between the new grid and the previous grid in these particular regions.
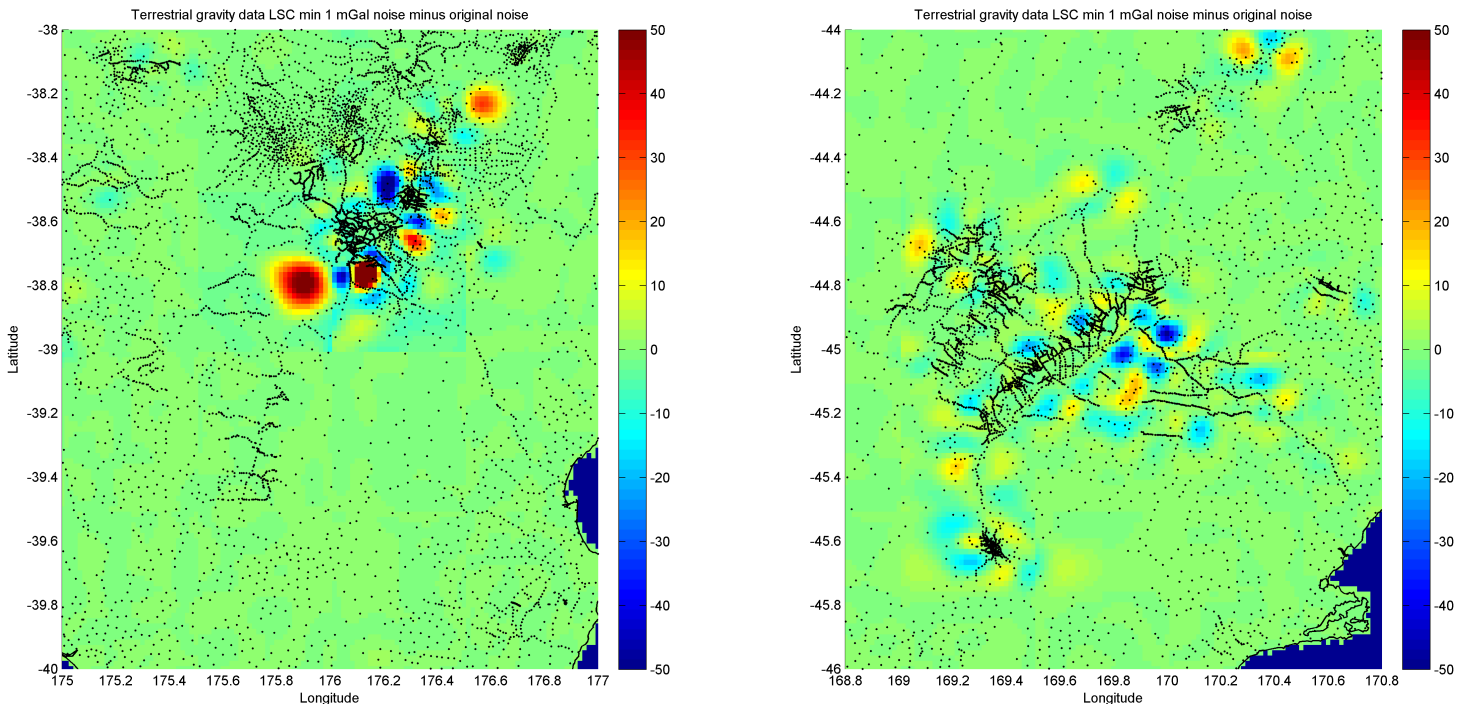

Figure 7.8: Gravity anomaly difference maps for the two regions. (New minus old) 
The big differences fall where the data has changed from being particularly dense and accurate to sparse. The previously prescribed small noise values must constrain the grid too much around individual points, resulting in spikes in transition areas. On average, on a nation wide scale, increasing the minimum noise level to $1 \mathrm{mGal}$ appears to have a negligible effect and only moderately smooths the gridded gravity field, as can be seen in the following difference map between the new and previous gridded gravity anomaly and error maps, since all of the features are high frequency. 

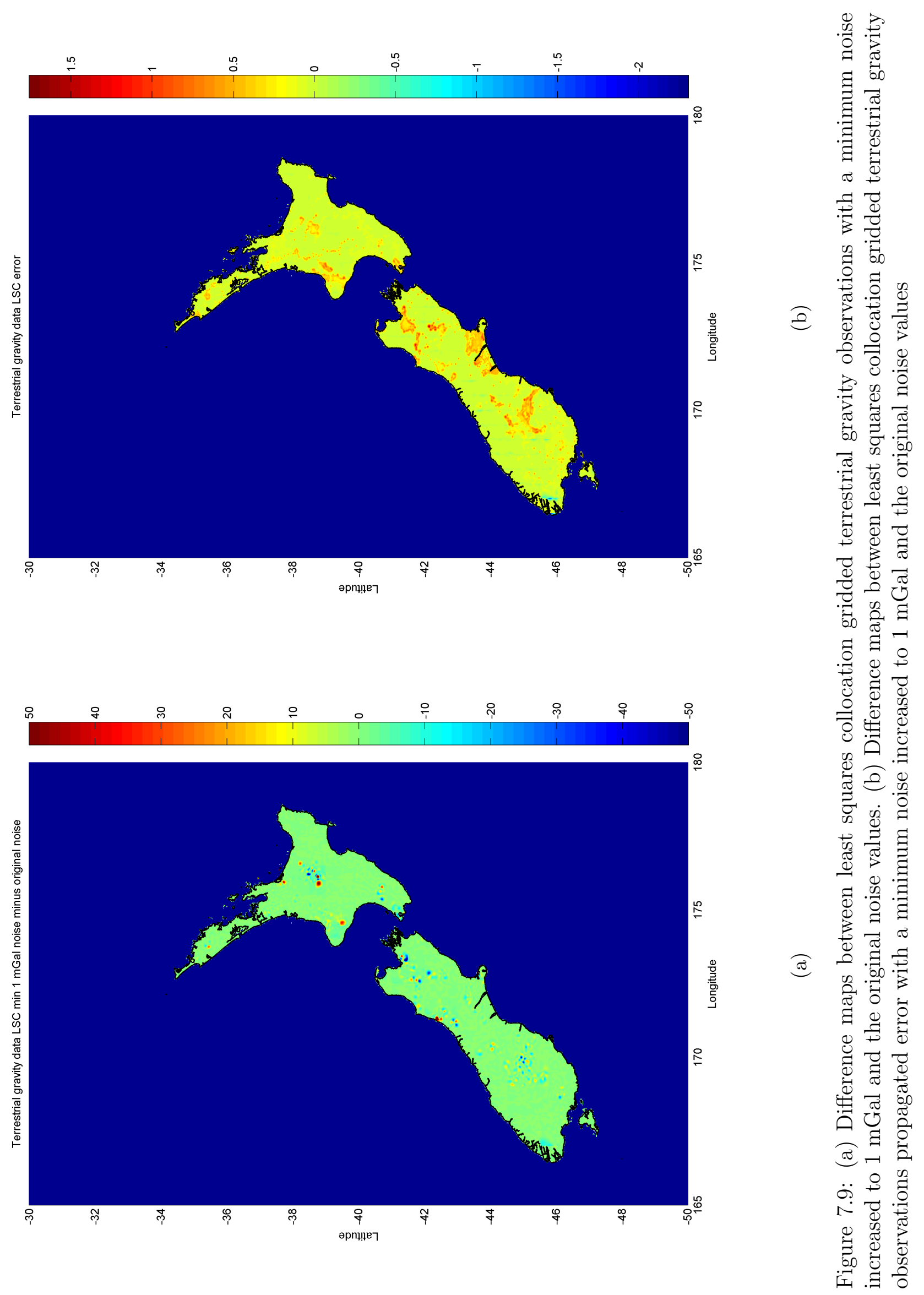
The airborne gravity data: The airborne gravity data are discussed in section 6.1. Least squares collocation with the logarithmic covariance function has been used to transform the along track gravity observations above the surface of the Earth, into a regular grid of gravity anomalies downward continued to the topographic surface. In section 6.5, the logarithmic covariance parameters for the airborne gravity data after removing the GOCE gravity anomaly were determined to be $C_{0}=576 \mathrm{mGal}, D=23.5 \mathrm{~km}$ and $T=46.5 \mathrm{~km}$ and the noise level was estimated to have a standard deviaiton of around $3 \mathrm{mGal}$.

A typical assumption of least squares collocation is that the noise in the input data is white. However, due to the along track filtering required for airborne gravity data reduction, observations are not independent along flight lines. The false assumption results in unrealistic error propagation where the data are dense along a single line. The off diagonal elements of the noise matrix have been estimated by considering the covariance between successive filtered gravity observations.

The low pass filter which was used to reduce the along track noise in the time domain was a Gaussian window function (with a half width of $k$ ) of the form,

$$
\bar{\Delta} g_{n}=\frac{\sum_{i=n-k}^{n+k} w_{i} \Delta g_{i}}{\sum_{i=n-k}^{n+k} w_{i}}
$$

where $\Delta g_{i}$ are the raw gravity observations along a fight line before filtering (which are assumed to contain additive white noise with variance $\sigma^{2}$ ) and $w_{i}$ are the weights of the window function. This means that that variance of the additive white noise in the $\bar{\Delta} g_{n}$ 's, denoted $\bar{N}_{n}$, is related to the variance of noise in the $\Delta g_{n}$ 's, denoted $N_{n}$, by the following,

$$
\begin{gathered}
\operatorname{var}\left(\bar{N}_{n}\right)=\operatorname{var}\left(\frac{\sum_{i=n-k}^{n+k} w_{i} N_{i}}{\sum_{i=n-k}^{n+k} w_{i}}\right) \\
\Longrightarrow \operatorname{var}\left(\bar{N}_{n}\right)=\frac{1}{\left(\sum_{i=n-k}^{n+k} w_{i}\right)^{2}} \operatorname{var}\left(\sum_{i=n-k}^{n+k} w_{i} N_{i}\right) \\
\Longrightarrow \operatorname{var}\left(\bar{N}_{n}\right)=\frac{\sum_{i=n-k}^{n+k} w_{i}^{2}}{\left(\sum_{i=n-k}^{n+k} w_{i}\right)^{2}} \operatorname{var}\left(N_{i}\right) \\
\Longrightarrow \operatorname{var}\left(\bar{N}_{n}\right)=\sigma^{2} \frac{\sum_{i=n-k}^{n+k} w_{i}^{2}}{\left(\sum_{i=n-k}^{n+k} w_{i}\right)^{2}}
\end{gathered}
$$

Then for some time lag $t$,

$$
\operatorname{var}\left(\bar{N}_{n+t}\right)=\sigma^{2} \frac{\sum_{i=n+t-k}^{n+t+k} w_{i}^{2}}{\left(\sum_{i=n+t-k}^{n+t+k} w_{i}\right)^{2}}
$$

and the covariance between $\bar{N}_{n}$ and $\bar{N}_{n+t}$ is given by (with the expectation of $\bar{N}_{n}$ and $\bar{N}_{n+t}$ zero),

$$
\begin{aligned}
& \Longrightarrow \operatorname{covar}\left(\bar{N}_{n}, \bar{N}_{n+t}\right)=E\left(\frac{\sum_{i=n-k}^{n+k} w_{i} N_{i}}{\sum_{i=n-k}^{n+k} w_{i}} \times \frac{\sum_{j=n+t-k}^{n+t+k} w_{j} N_{j}}{\sum_{j=n+t-k}^{n+t+k} w_{j}}\right) \\
& \Longrightarrow \operatorname{covar}\left(\bar{N}_{n}, \bar{N}_{n+t}\right)=E\left(\frac{\sum_{i=n-k}^{n+k} \sum_{j=n-k}^{n+k} w_{i} N_{i} w_{j+t} N_{j+t}}{\sum_{i=n-k}^{n+k} \sum_{j=n-k}^{n+k} w_{i} w_{j+t}}\right)
\end{aligned}
$$




$$
\Longrightarrow \operatorname{covar}\left(\bar{N}_{n}, \bar{N}_{n+t}\right)=\frac{\sum_{i=n-k}^{n+k} \sum_{j=n-k}^{n+k} w_{i} w_{j+t} E\left(N_{i} N_{j+t}\right)}{\sum_{i=n-k}^{n+k} \sum_{j=n-k}^{n+k} w_{i} w_{j+t}}
$$

since it was assumed that $N_{i}$ and $N_{i+t}$ are independent,

$$
E\left(N_{i} N_{j+t}\right)= \begin{cases}0 & : i \neq j+t \\ \sigma^{2} & : i=j+t\end{cases}
$$

then $\operatorname{covar}\left(\bar{N}_{n}, \bar{N}_{n+t}\right)$ is given by,

$$
\operatorname{covar}\left(\bar{N}_{n}, \bar{N}_{n+t}\right)=\sigma^{2} \frac{\sum_{j=n+t-k}^{n+k} w_{j}^{2}}{\sum_{i=n-k}^{n+k} \sum_{j=n-k}^{n+k} w_{i} w_{j+t}}
$$

The covariance for the Gaussian filter applied to the airborne data has a standard deviation parameter of 120 seconds (see section 5). Figure 7.10 shows this function normalized by the variance, $\operatorname{var}\left(\bar{N}_{n}\right)$, plotted against a time lag $t$.

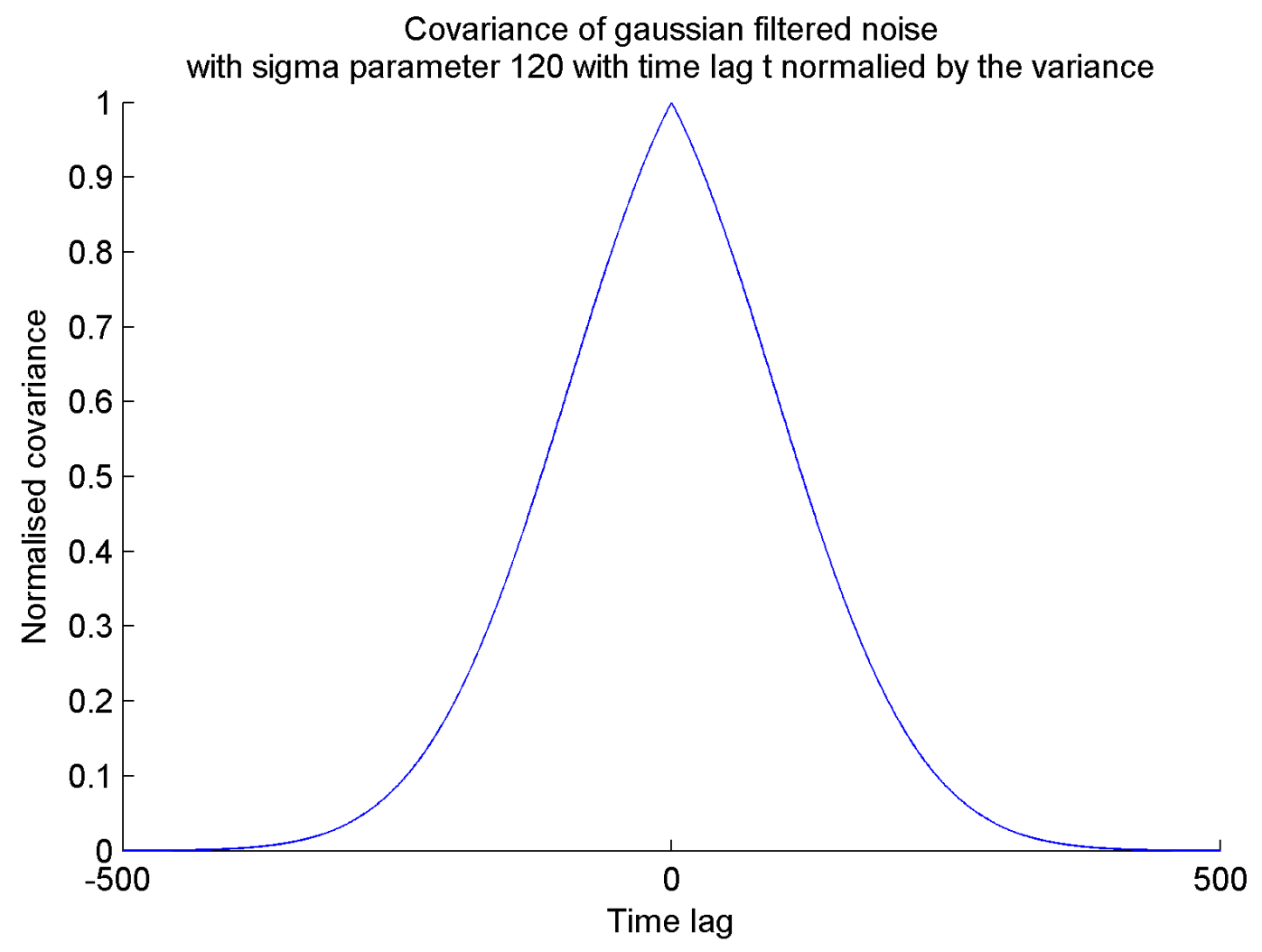

Figure 7.10: Covariance of Gaussian filtered noise with $\sigma$ parameter 120 with time lag t.

Figures 7.11, 7.12 and 7.13 show the airborne data gridded by least squares collocation at the topographic surface and the propagated error map for; the noise matrix being strictly diagonal, the noise matrix with the covariance values, and the difference between them. Data which have been assigned a zero weight in section 6.4 have been excluded and this is reflected in the error propogation. 

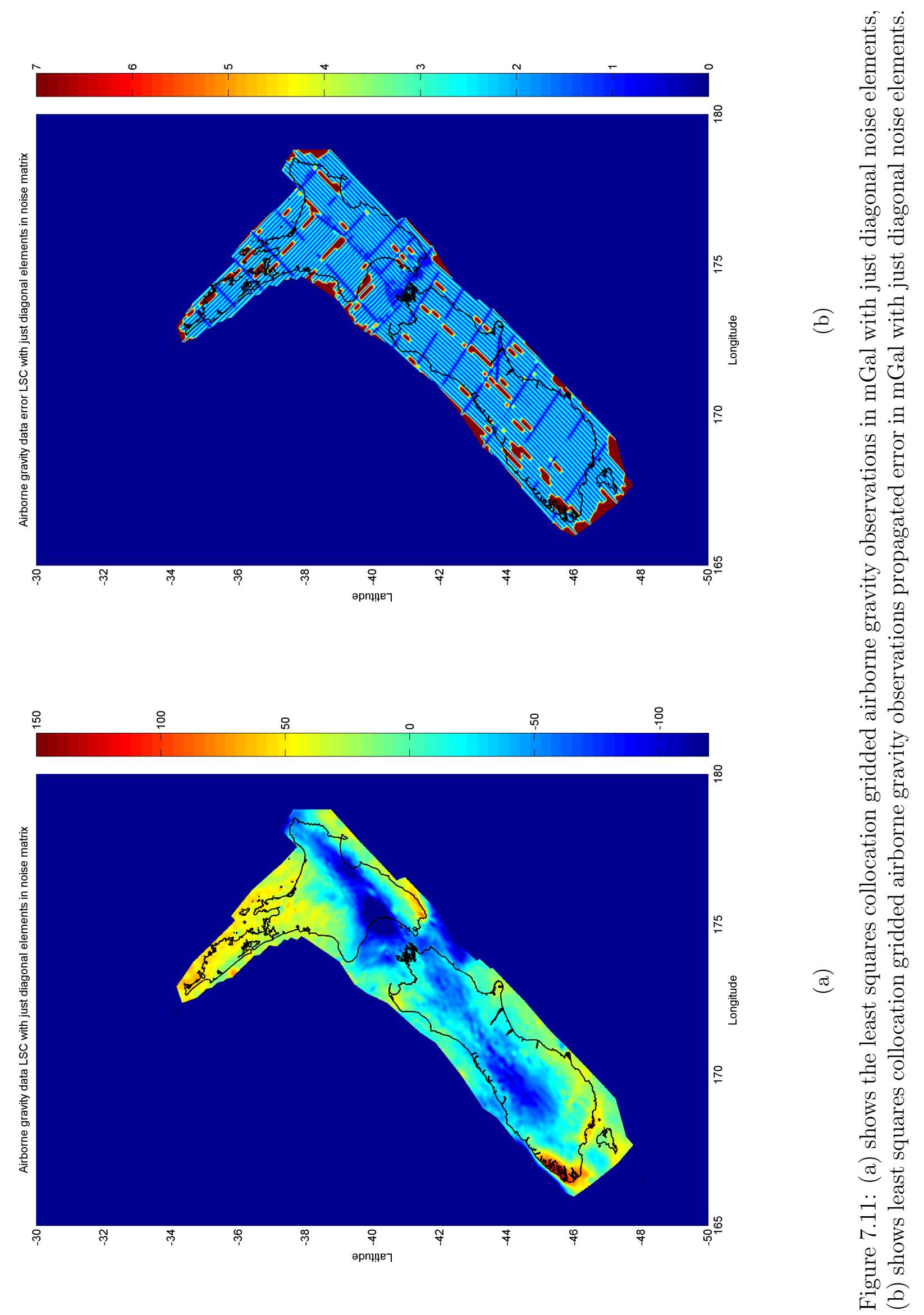

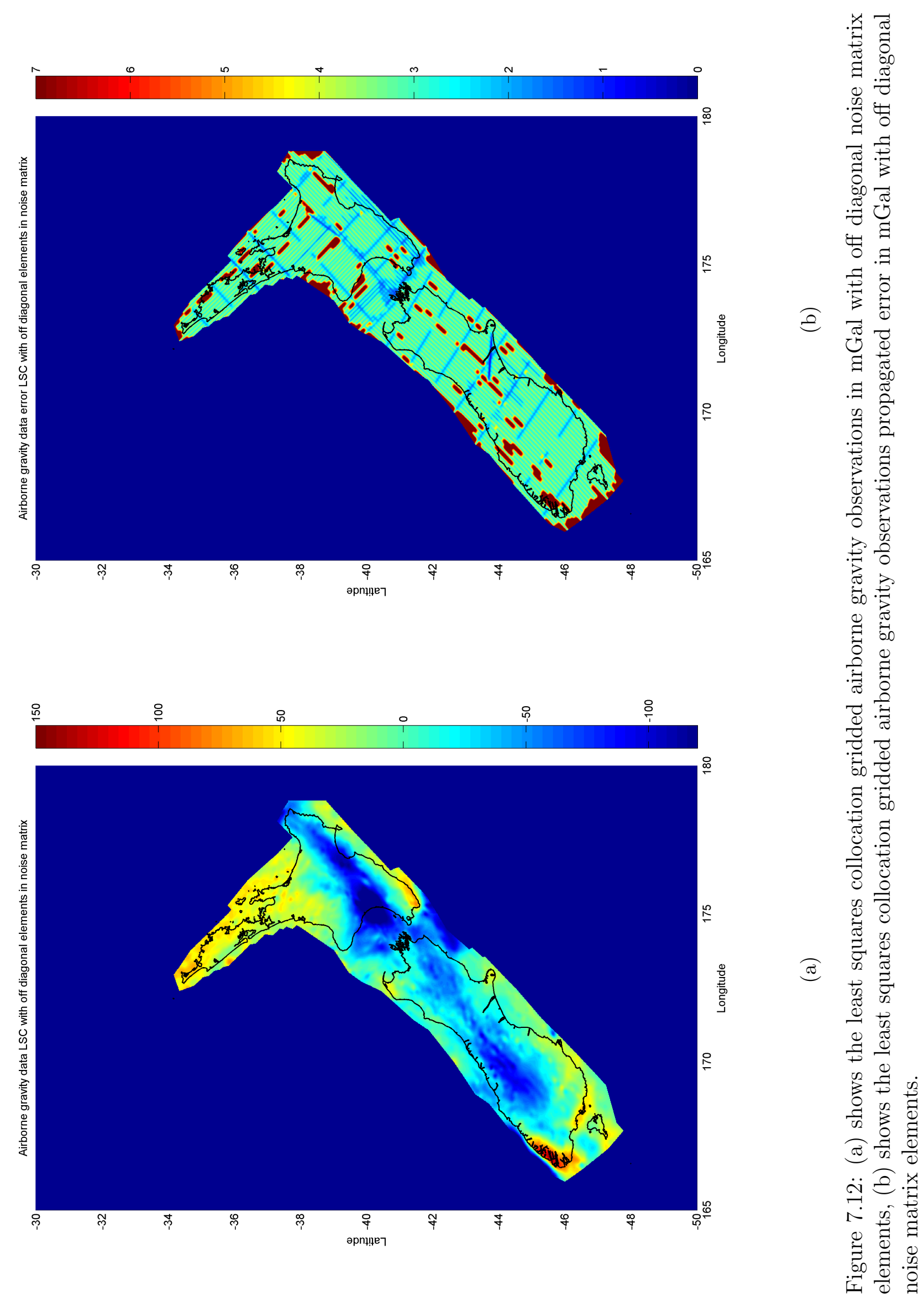

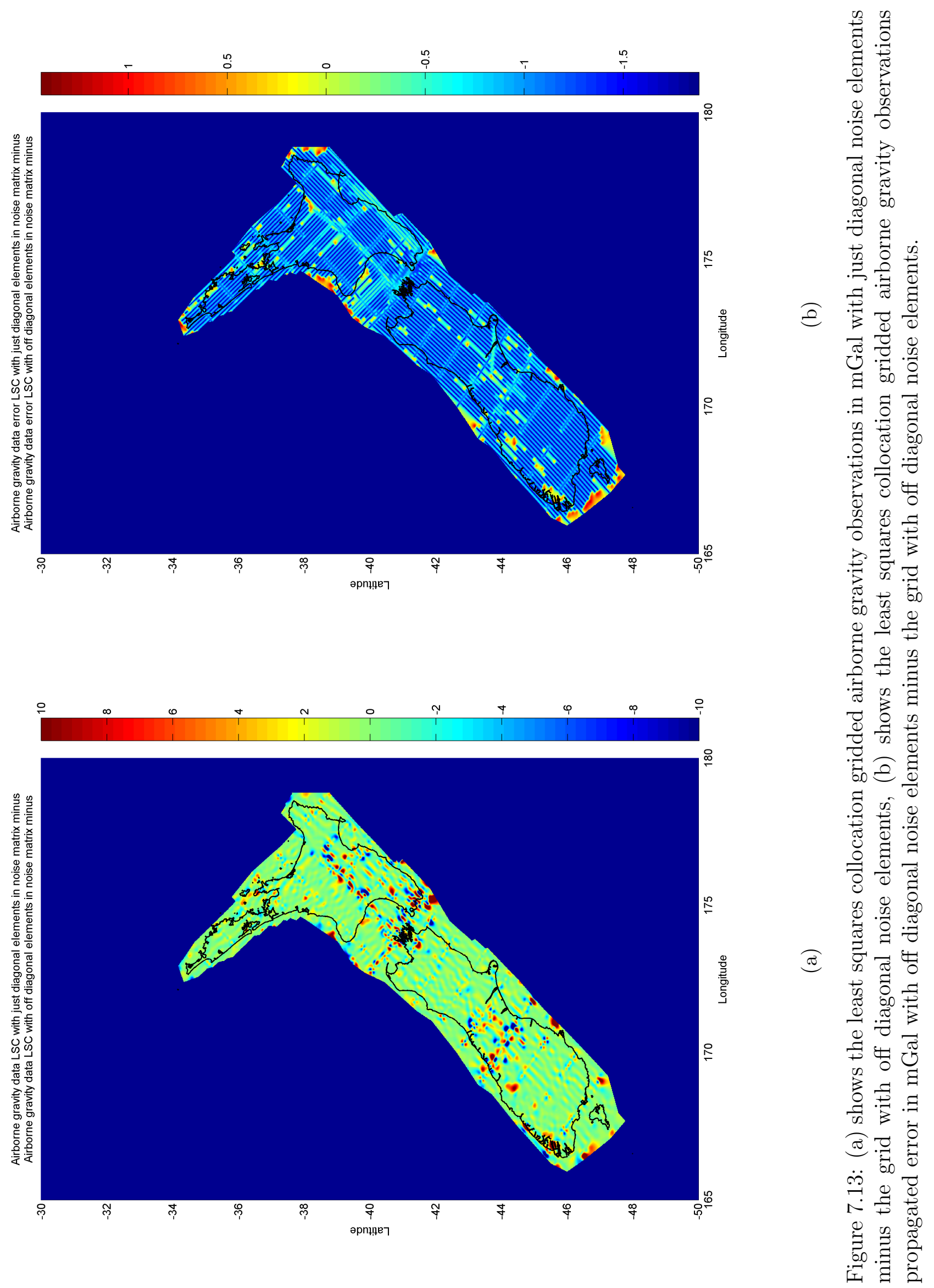
On a national scale the differences appear to be reasonably small, and typically between - 10 and $+10 \mathrm{mGal}$. However on a smaller scale, using off diagonal elements in the noise matrix results in smoother contours and removes artifacts which are not particularly well correlated spatially (i.e. across neighboring flight lines).

Figure 7.14a shows the gravity anomaly in and around the Taranaki Bight using just a diagonal noise matrix and figure 7.14b shows the same thing using off diagonal noise matrix elements. The flight lines have been overlaid on the maps to show where the poorly spatially correlated features along individual flight lines are removed. This is most noticeable in the gridded anomalies in the South Wairarapa District in the South-East.

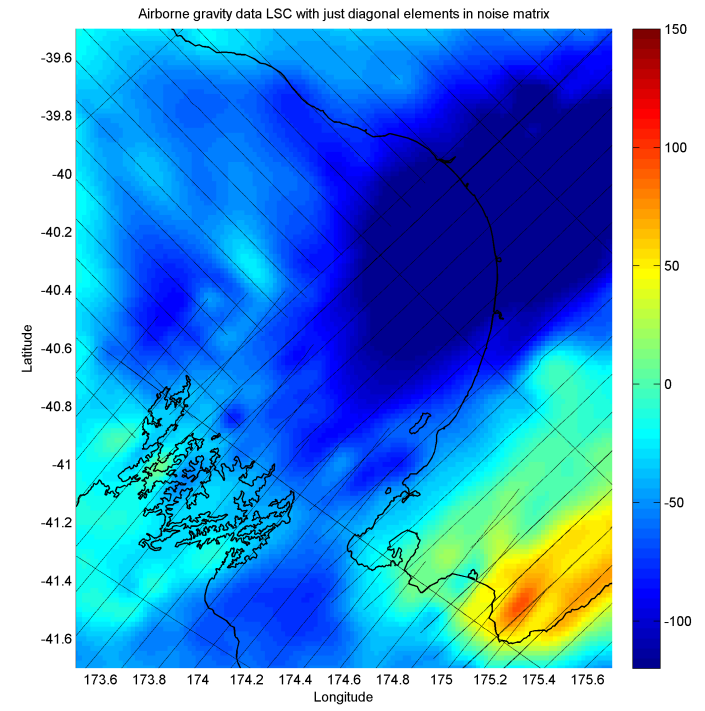

(a)

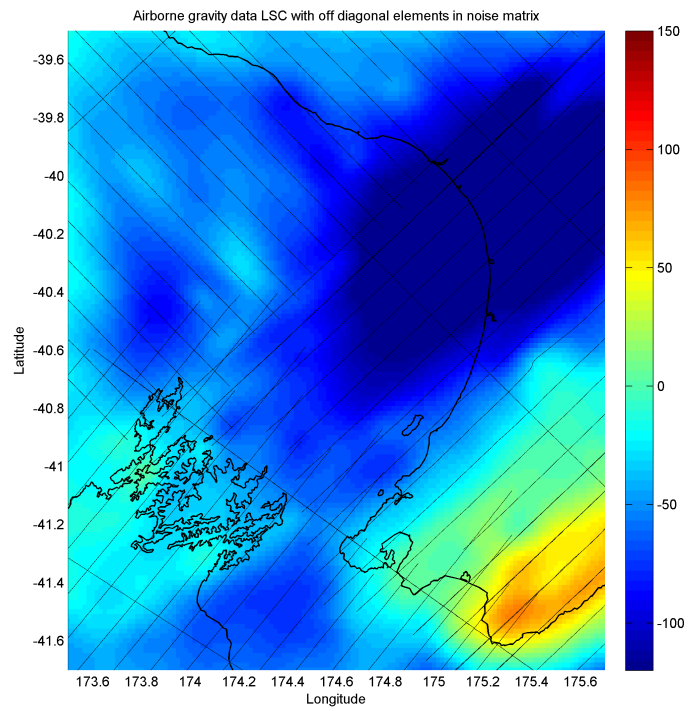

(b)

Figure 7.14: Least squares collocation gridded airborne gravity observations in mGal around the Taranaki Bight using just diagonal noise matrix elements (a) and off diagonal noise matrix elements (b).

This amended form of the noise matrix for the airborne data is a crucial step to appropriately combine airborne and terrestrial gravity data since otherwise the high data density of the airborne data will cause the gridded gravity data to be unfairly biased towards it, drastically restricting the influence of the terrestrial gravity observations.

The effect of combining the airborne and terrestrial gravity data: The airborne and terrestrial gravity anomalies have been combined using least squares collocation (with the logarithmic covariance parameters derived for the covariance of the airborne data alone) and the gridded gravity anomaly and propagated error map can be seen in figures $7.15 \mathrm{a}$ and 7.15b. Looking at the map of propagated errors it is clear that the least squares collocation pulls on the strengths of both data sets. The terrestrial data is (in general) highly accurate although it is restricted (on the whole) to onshore areas and has varying density, the contribution of the airborne data to the grid is to provide consistently dense on and off shore data coverage with a (prior) 3mGal accuracy. This results in a propagated error map which is more uniform in comparison to just the terrestrial data, more accurate than the airborne alone, and a gravity anomaly map which is seamless between on and off shore areas. 

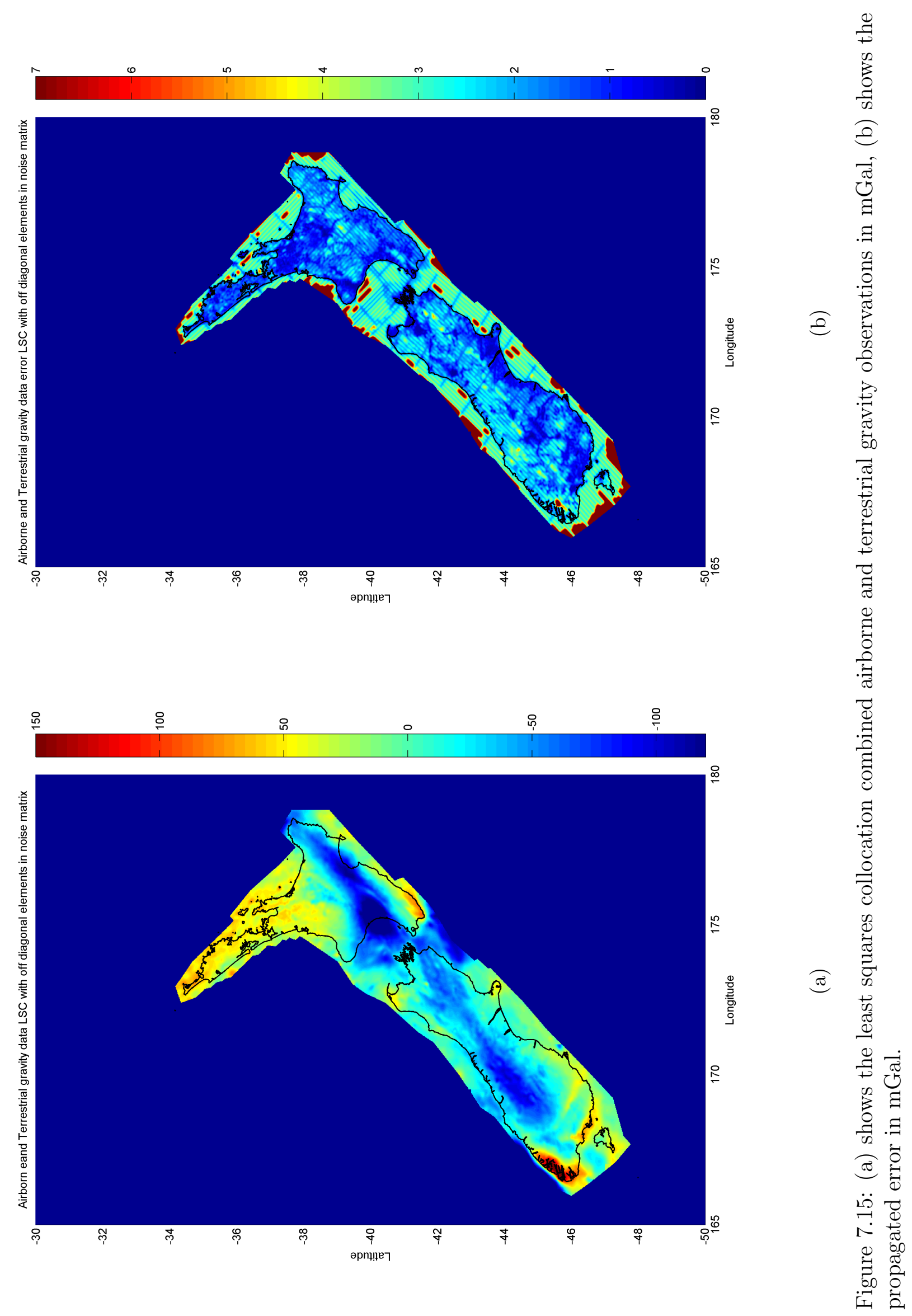
The Shipborne gravity data: Marine gravity observations have been collected in the New Zealand continental region over a time period spanning 50 years. The measurements have been made by various institutions (UNCLOS, GNS Science, NOAA and GA) primarily for mineral exploration. The database contains around 2 million individual observations and figure 7.16 shows where these data have been obtained in the region $160(\mathrm{E})$ to $190(\mathrm{E})$ and $25(\mathrm{~S})$ to $60(\mathrm{~S})$. The database of measurements contains gravity values, horizontal coordinates (latitude and longitude in terms of the GRS80 reference ellipsoid), normal gravity and Eotvos corrections.

A cross-over adjustment was performed on the ship track gravity data to remove potential offsets between separate gravity surveys and individual lines of data (as identified by Wessel and Watts 1988). This was performed by Intrepid Geophysics (Amos et al, 2005) and allowed several separate datasets to be combine into a single set of self consistent measurements. The data were also further cleaned by removing any spikes and obviously anomalous data.

Initially, prior to the cross-over adjustment, the cross-over error was evaluated to have a standard deviation of around $2 \mathrm{mGal}$. This can be seen in table 7.2 of the cross-over statistic for each set of gravity observations treated separately for each institution, and as a whole. This was reduced to approximately $0.3 \mathrm{mGal}$ after the cross-over adjustment as can be seen in table 7.3 .

Table 7.2: The cross-over statistics for the shipborne gravity data prior to the cross-over adjustment - adapted from Amos (2007) appendix C.

$\begin{array}{llllll}\text { Data } & \text { Cross-overs } & \text { Max } & \text { Min } & \text { Mean } & \text { STD } \\ \text { UNCLOS } & 345 & 79.7 & 0 & 7.6 & 12.9 \\ \text { GNS } & 57512 & 271.3 & 0 & 2.5 & 7.6 \\ \text { NOAA } & 971988 & 236.1 & 0 & 0.7 & 0.7 \\ \text { GA } & 36271 & 52.6 & 0 & 1.6 & 2.7 \\ \text { All data } & 1069289 & 271.3 & 0 & 0.8 & 2\end{array}$

Table 7.3: The cross-over statistics for the shipborne gravity data after the cross-over adjustment - adapted from Amos (2007) appendix C.

$\begin{array}{llllll}\text { Data } & \text { Cross-overs } & \text { Max } & \text { Min } & \text { Mean } & \text { STD } \\ \text { UNCLOS } & 345 & 12.1 & 0 & 0.45 & 1.39 \\ \text { GNS } & 57512 & 68.9 & 0 & 0.19 & 1.5 \\ \text { NOAA } & 971988 & 1.9 & 0 & 0.09 & 0.08 \\ \text { GA } & 36271 & 14.9 & 0 & 0.04 & 0.11 \\ \text { All data } & 1069244 & 93.4 & 0 & 0.05 & 0.28\end{array}$

Cross-over adjustments, as identified in section 6.6, in general are poorly constrained problems and so the cross-over standard deviation after the adjustment does not necessarily reflect the accuracy of the data. i.e. if each line only had one intersection point, the cross-over difference after an adjustment would be exactly zero for all intersections. For this reason the shipborne data has been assigned a prior $2 \mathrm{mGal}$ standard deviation error estimate for the least squares collocation griding. 


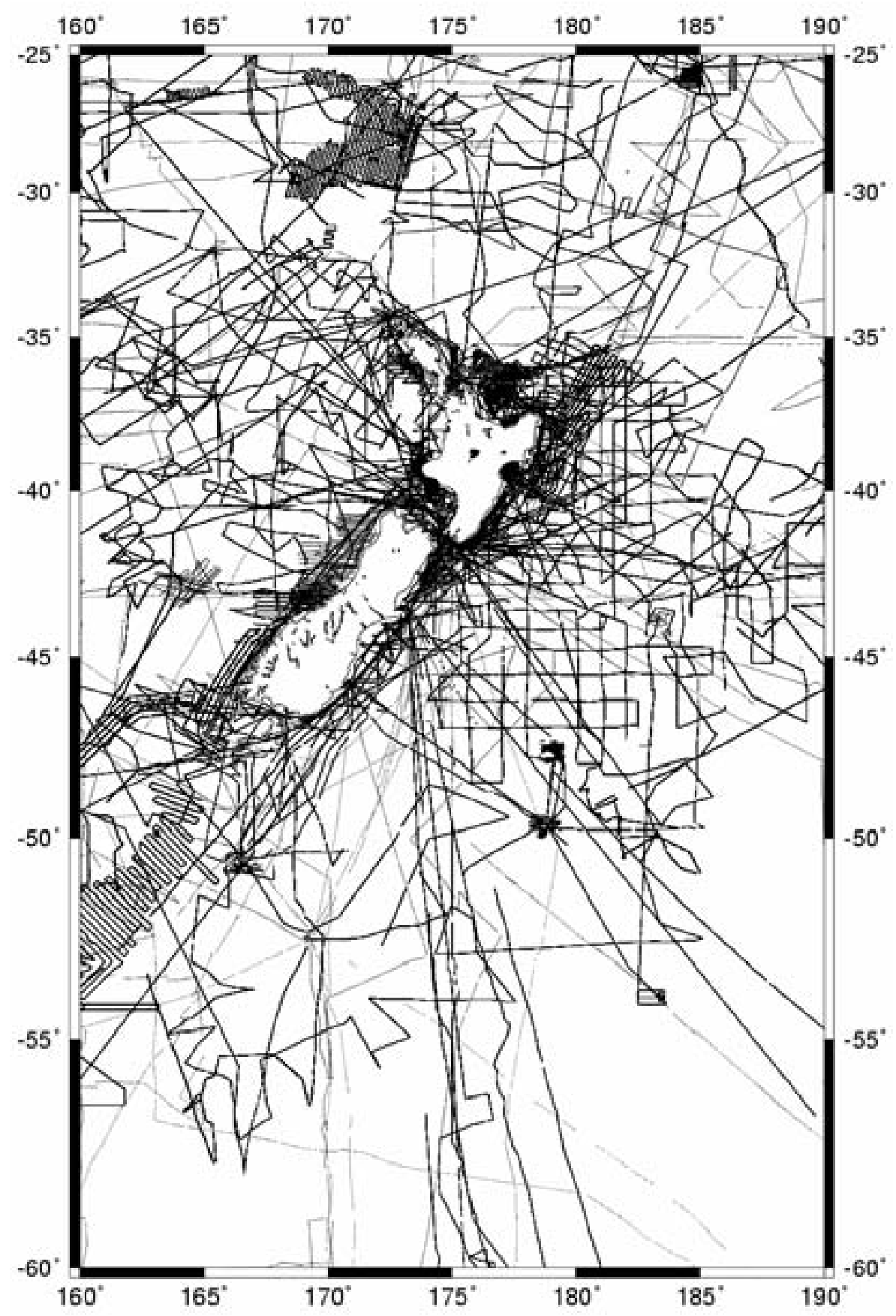

Figure 7.16: Ship track data coverage in the region 160 (E) to 190 (E) and 25 (S) to 60(S). Adapted from Amos (2007) appendix C. 
The Satellite gravity data: A satellite marine gravity anomaly grid (at one arc minute) released in 2014 by Sandwell et al (2014) has been obtained from http://topex.ucsd.edu/cgibin/get_data.cgi (last accessed September 2015). The grid is based on data from two new satellite altimeters, CryoSat-2 and Jason-1 (contributing over 70 months of data) which have been augmented with older altimeter data from Geosat and ERS-1. The augmentation is reported to have resulted in an improvement of a factor of 2-4 in the marine gravity field ( Sandwell et. al, 2014).

CrySat-2 's primary objective is to measure changes in sea ice to approximately $0.7 \mathrm{~cm}$. It has dense data coverage and, after 4 years in operation, a nominal track spacing of approximately $2.5 \mathrm{~km}$. Jason-1's mission objectives are to provide a 5-year view of global ocean surface topography and increase the understanding of ocean circulation. It has provided 14 months of dense track coverage with a track spacing of $7.5 \mathrm{~km}$. Geosat (U.S Navy Geodetic Satellite), was launched in 1985 and carried an altimeter that could measure it's relative position to the ocean to an accuracy of around $5 \mathrm{~cm}$. After a brief period on a classified mission, it's scientific mission began in November 1986 and ended in January 1990 resulting in 3 years of $5 \mathrm{~cm}$ accurate altimeter data which are available to the scientific community. ERS-1 (European remote sensing), was launched in July 1991 and provided altimeter data for a 9 year period.

The altimeter derived gravity field is estimated to be accurate to around $2 \mathrm{mGal}$. The improvements in the estimated gravity field, over the older altimeter data, occurs mostly in the 12 - $40 \mathrm{~km}$ wavelengths. Over the land areas, gravity anomalies from EGM2008 have been used, which are based on a (approximately) $10 \mathrm{~km}$ grid of terrestrial gravity observations. The supplied grid is shown in figure 7.17 over the latitude range of $25(\mathrm{~S})$ to $60(\mathrm{~S})$ and longitudes from $160(\mathrm{E})$ to $190(\mathrm{E})$.

On a visual inspection of the map, smaller errors appear to fall directly along the path of the satellite and in general, in the open ocean, the error is around 1-2 mGal with small variability. In shallow coastal areas the error increases up to $50 \mathrm{mGal}$. On shore the data have been taken from EGM2008 and have been given a zero error.

To remove the EGM2008 coastal and on shore data in the satellite grid, data inside a coastline mask of the North Island, South Island, Stewart Island and Chatham Island have been removed from the grid. The data in shallow coastal areas have much larger (gridded) noise values; these gridded noise values will be used in the least squares collocation process, to assign Gaussian white noise to the matrix $N$. The larger noise values on the coast will reduce the influence of these data on the combined least squares collocation grid.

The satellite gravity grid has an accompanying map of estimated errors. (Fenoglio et al 2008) In the open oceans the error is approximately $2 \mathrm{mGal}$ as described in the documentation (Sandwell et al, 2014) however in shallow coastal areas the accuracy drops predominately due to large sea surface variability (Chein and Hsin, 2008). The map of estimated errors is shown in figure 7.18.

Figure 7.19 shows the mask applied to the error map and figure 7.20 shows the gravity anomaly map with marks indicating where the airborne, terrestrial and shipborne gravity data are. There is good data coverage, from the terrestrial, airborne and shipborne data, where the satellite data have been removed and in transitional areas. 


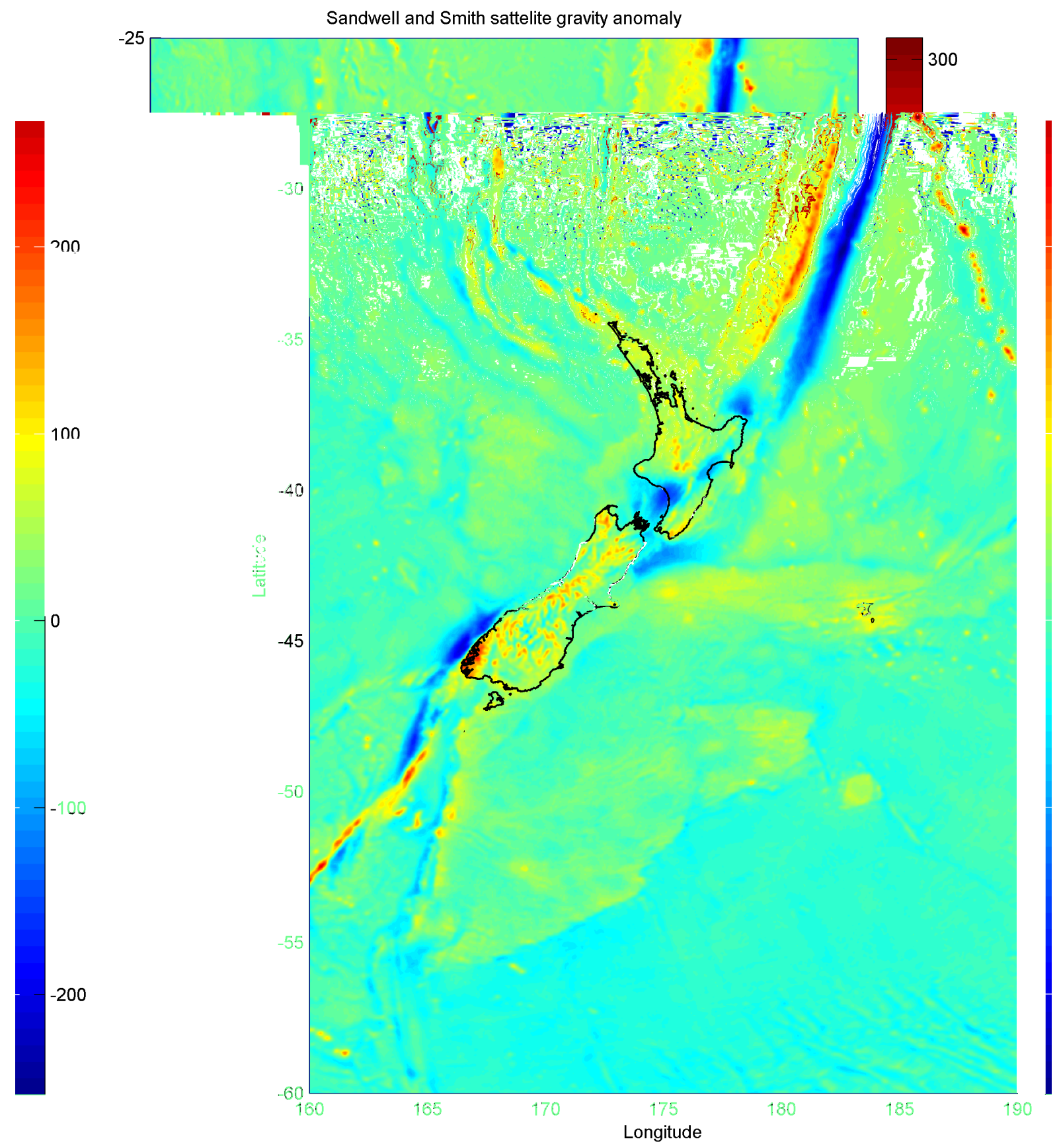

Figure 7.17: 1 arc minute gridded Sandwell et al (2014) gravity anomaly in mGal. 


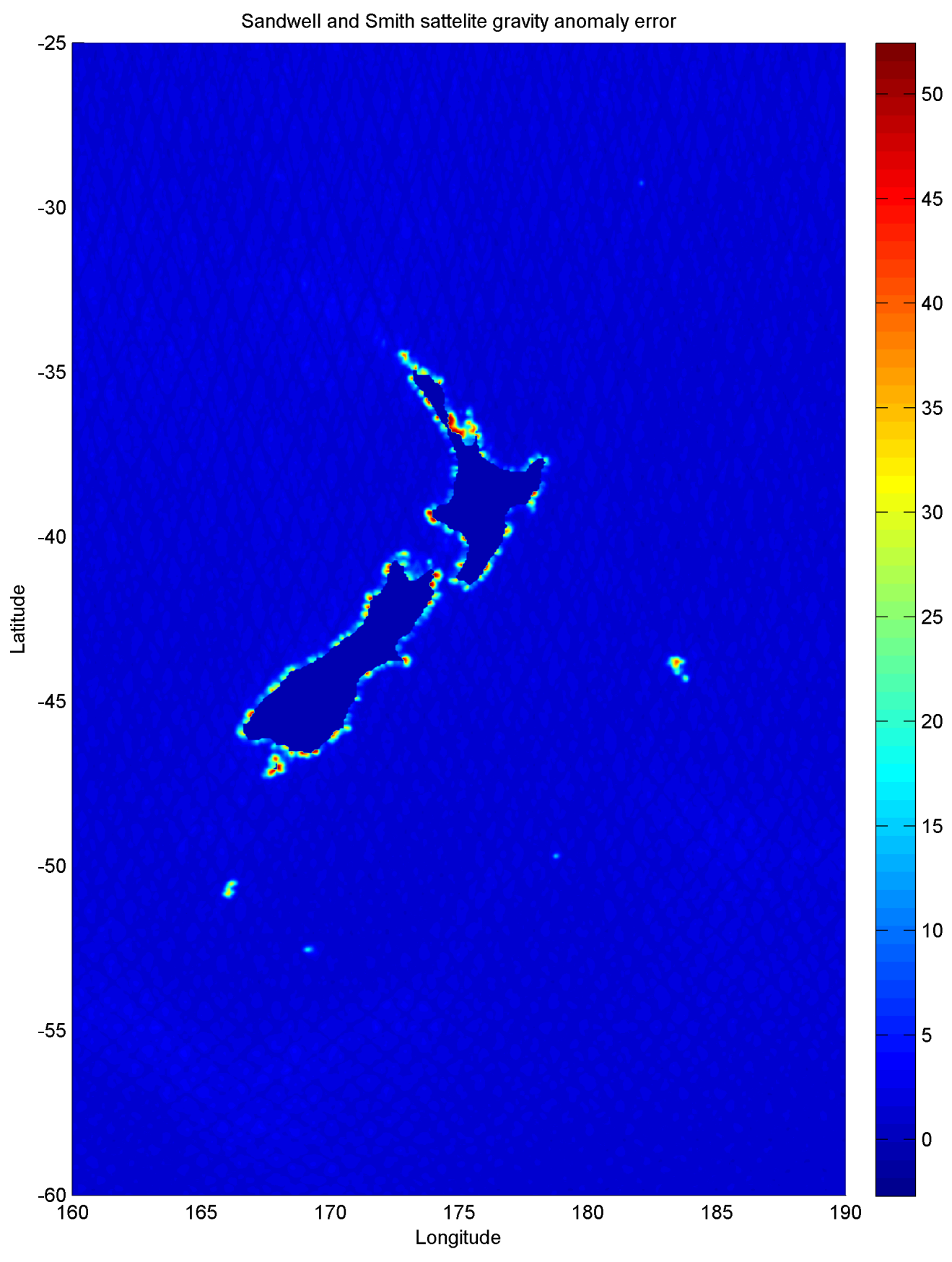

Figure 7.18: 1 arc minute gridded Sandwell et al (2014) gravity anomaly error in mGal. 


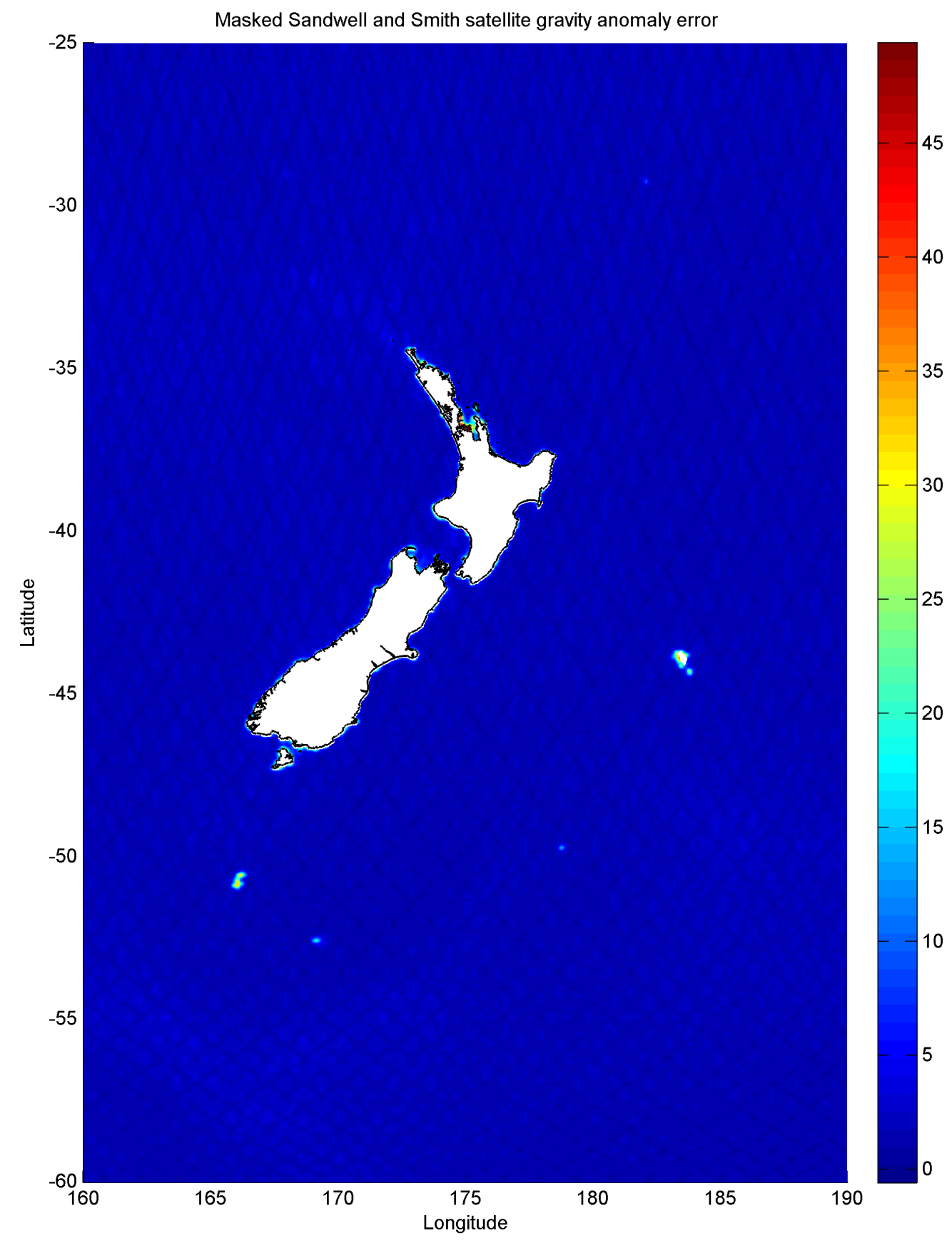

Figure 7.19: 1 arc minute gridded Sandwell et al (2014) gravity anomaly error in mGal masked onshore and near offshore. 


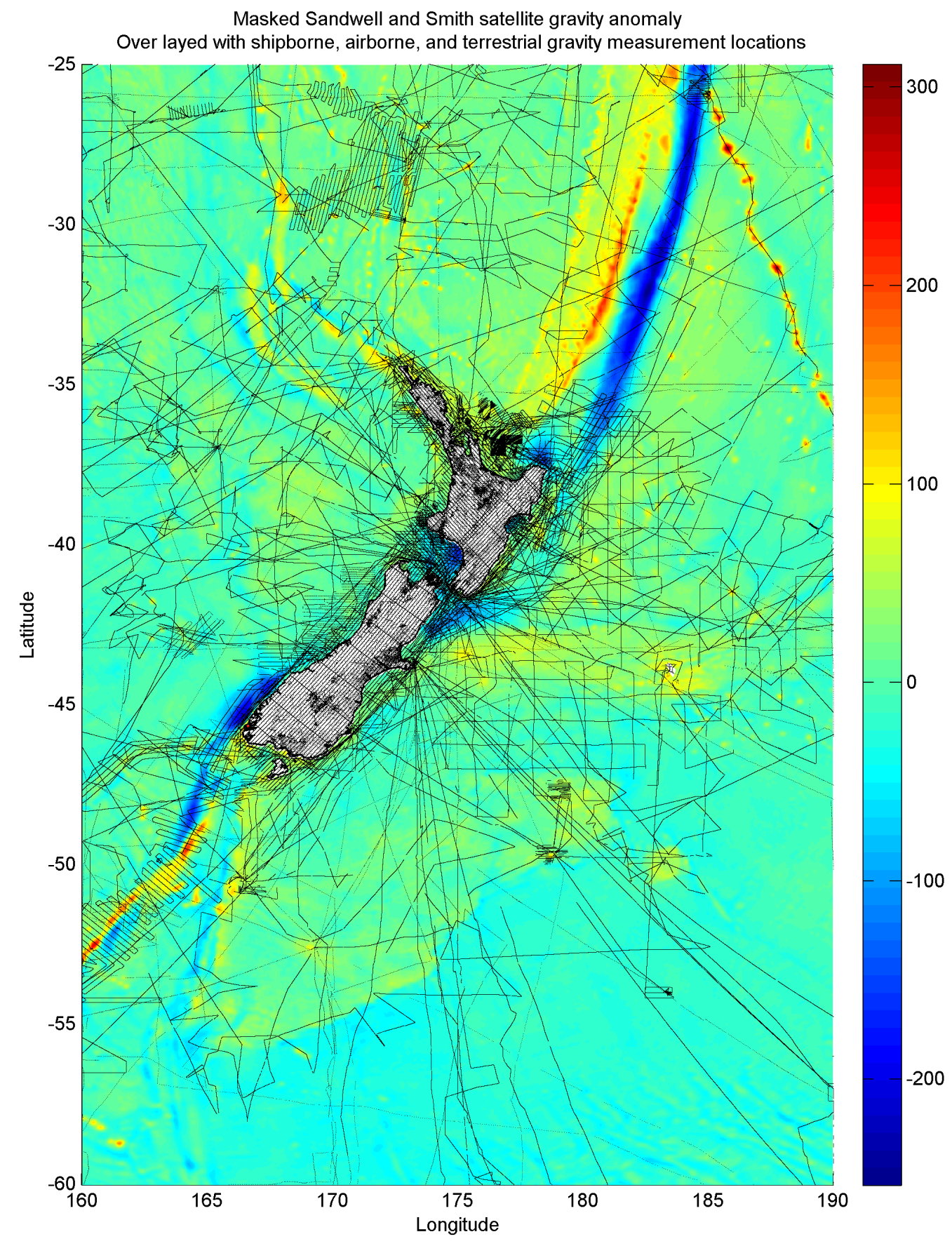

Figure 7.20: 1 arc minute gridded Sandwell et al (2014) gravity anomaly masked with indicative marks (in black) where the other data are. There is good data coverage, from the terrestrial, airborne and shipborne data, where the satellite data have been removed and in transitional areas. 
A combined gravity anomaly grid at the topographic surface using all available gravity data: The airborne, terrestrial, shipborne and satellite gravity data have been combined into a single data set and gridded using least squares collocation. The final gridded gravity values and propagated error standard deviations (taking the square root of the diagonal entries of $E_{s s}$ ) are given at the 1 arc minutes locations shown in figure 7.1. The airborne data were given a prior noise value of $3 \mathrm{mGal}$ standard deviation, the terrestrial noise level is as described previously, the shipborne data have been assigned a $2 \mathrm{mGal}$ standard deviation and the supplied satellite error estimates have been used. Figure 7.21 shows the gridded gravity anomaly and figure 7.22 shows the propagated error map.

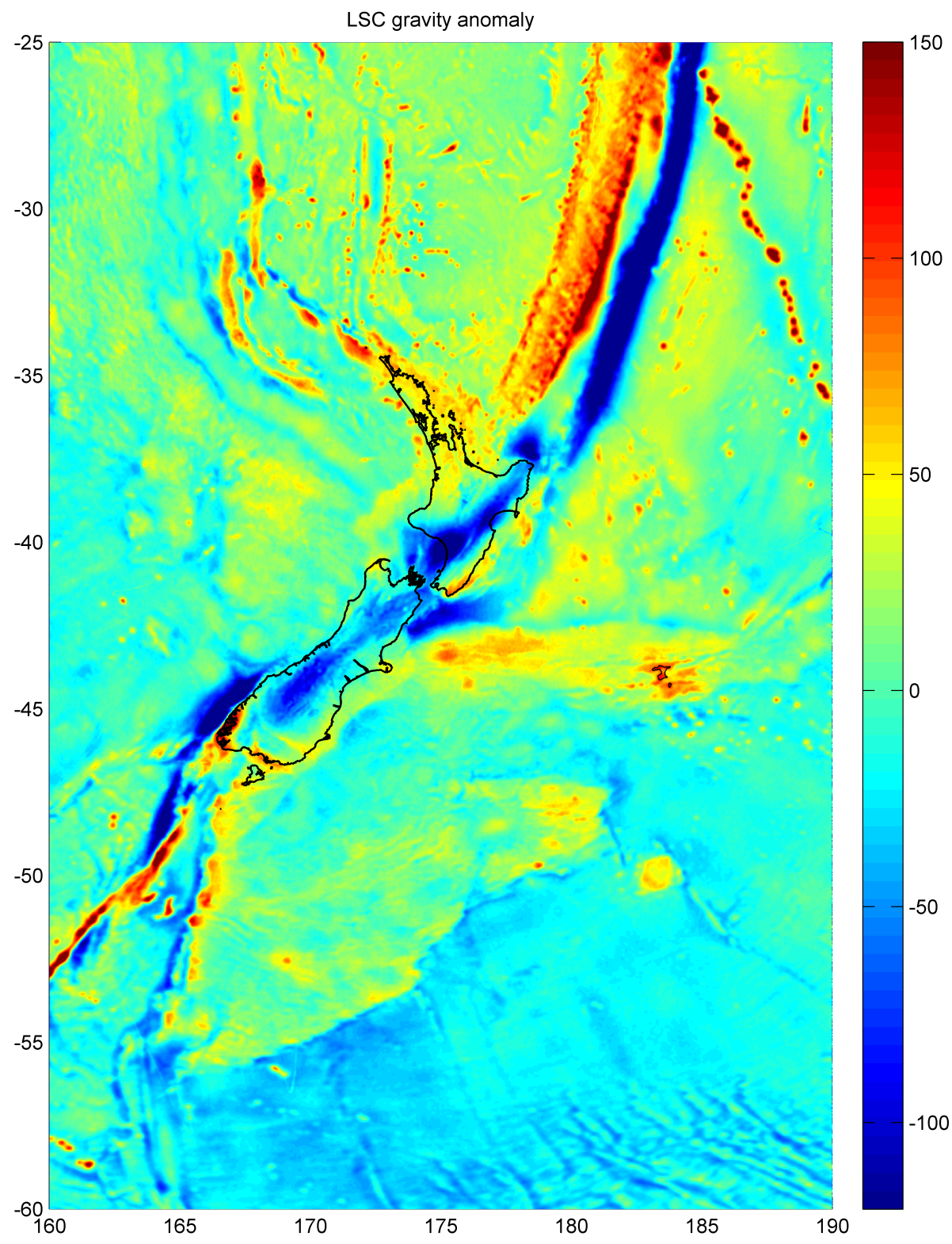

Figure 7.21: Topography corrected gravity anomaly map in the region 160 (E) to 190 (E) and $25(\mathrm{~S})$ to $60(\mathrm{~S})$ gridded using least squares collocation using the airborne, terrestrial, shipborne and satellite gravity data. The scale is in mGal. 


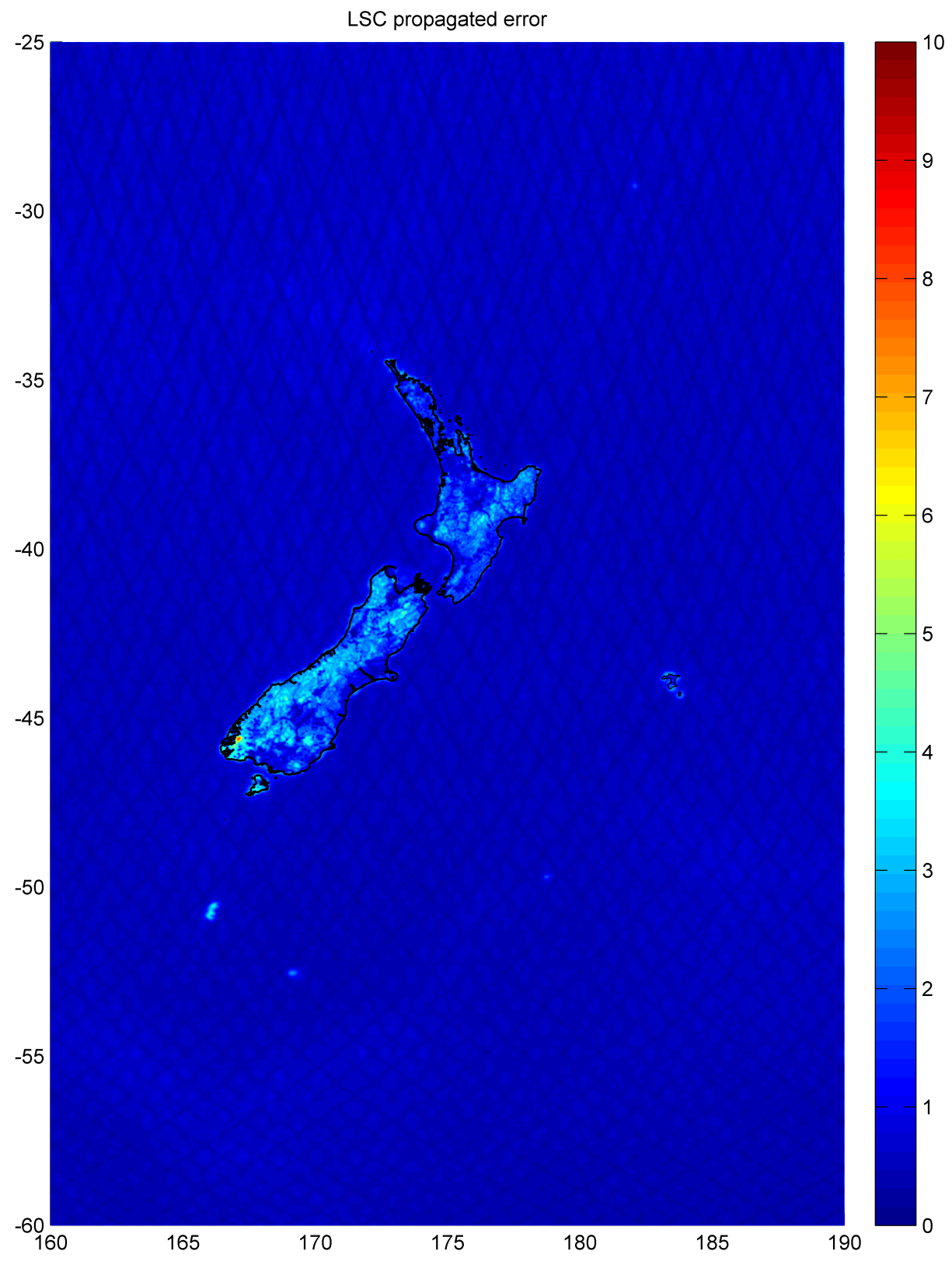

Figure 7.22: Gravity anomaly propagated error map in the region 160 (E) to 190 (E) and $25(\mathrm{~S})$ to $60(\mathrm{~S})$ gridded using least squares collocation using the airborne, terrestrial, shipborne and satellite gravity data. The scale is in mGal.

\subsection{Summary}

This chapter discussed the method of least squares collocation and how it can be used to combine scattered gravity observations (of different accuracies) into a regular grid. The key methodology and equations for least squares collocation are given in section 7.2.

It has been shown that least squares collocation can be used to combine observations that have been made at varying elevations using the logarithmic covariance since it is 3 dimensional. 
Steps were taken to eliminate potentially erroneous artifacts in the gridded data by appropriately adjusting the terrestrial noise estimates, by setting a $1 \mathrm{mGal}$ minimum value, and it was shown how the effect of the along track filter on the observation errors for the airborne data can be incorporated into the least squares collocation formulae. Two new data sets have been introduced, the shipborne gravity data and a new high resolution satellite gravity anomaly from Sandwell et al (2014). Further steps have been taken to remove potentially erroneous coastal data from the satellite gravity data, and the accompanying error estimates of the satellite model have been used to heavily down weight the influence of gravity values of the satellite gravity model in shallow coastal areas on the least squares collocation grid.

A final grid of gravity anomalies and accompanying propagated error map has been presented. These have been estimated from all available gravity observation sources which have been optimally combined with a careful consideration of observation errors and an appropriate choice of covariance function. This grid will be used to calculate a new gravimetric quasi-geoid in the following chapter. 


\section{Chapter 8}

\section{Computation of a New Gravimetric Quasigeoid}

\subsection{Introduction}

A new gravimetric quasigeoid has been computed from the gridded gravity data shown in figure 7.21. Utilising the methodology described in section 2.5, Helmert's second method of condensation and the remove-compute-restore technique have been employed.

The topographic effect has first been restored to the gridded gravity data shown in figure 7.21, using the 1 arc minute block averaged heights from the $8 \mathrm{~m}$ DEM shown in figure 7.1, by performing a reverse Bouguer slab correction. These gridded data are compared to the gridded gravity data used to compute the quasigeoid NZGeoid2009, described in section 3.4.

In section 5.1 the Eigen-6C4 global gravity model fitted the leveling derived quasigeoid height anomalies better than any other global gravity model. For this reason this model was chosen to provide the long wave length gravity signal when performing the remove-compute-restore technique. i.e. the Eigen-6C4 gravity anomaly, expanded up to its maximum degree and order, has been subtracted from the gridded gravity data, Stokes integral equation has been used to determine residual quasigeoid height anomalies from the residual gravity signal, then the Eigen-6C4 quasigeoid height anomalies have been added back to the residual quasigeoid height anomalies.

Stokes integration has been performed with the Featherstone et al (1998) modified Stokes Kernel since it was identified to be theoretically optimal. The modification degree $L$ and spherical cap $\psi$ have been varied over the range $L=20,40, \ldots, 320$ and $\psi_{0}=1^{\circ}, 1.5^{\circ}, 2^{\circ}, 2.5^{\circ}, 3^{\circ}$. After restoring the primary indirect topographical effect (described in section 2.5 ) and adding back the long wavelength quasigeoid height anomalies from the Eigen-6C4 global gravity model the new geoids have been compared to the leveling-derived quasigeoid height anomalies to determine the optimal $L$ and $\psi$ parameter combination.

It is shown that the quasigeoid determined using the optimal $L$ and $\psi$ parameter combination is in better agreement with the leveling derived height anomalies than the current official quasigeoid, NZGeoid2009, and the quasigeoid height anomalies determined from the Eigen-6C4 global gravity model. For this reason implementing a vertical datum with the newly derived regional quasigeoid height anomalies as the reference surface will provide more accurate normal- 
orthometric heights than presently possible.

\subsection{The gridded gravity anomaly used to determine a new quasigeoid}

Section 2.5.1 identifies the initial steps which need to be taken to obtain a gridded gravity anomaly with the gravitational effect of topography reduced to the quasigeoid. First a gridded Bouguer anomaly must be obtained and then the effect of topography must be restored by performing an inverse Bouguer slab correction (described in section 2.2.3).

Figure 7.21 shows the gridded Bouguer gravity anomaly at the topographic surface and figure 7.1 shows the height of the topographic surface at the grid points. These heights $h$ have been used in equation 8.1 to obtain a grid of Bouguer slab corrections $\delta g_{B}$ which are then subtracted from the gridded Bouguer gravity anomaly.

$$
\delta g_{B}=-2 \pi \rho G h=-0.0419 \rho h
$$

where $\rho$ is the density of the topography taken as $2.67 \mathrm{~g} / \mathrm{cm}^{3}$.

This results in a gridded Faye anomaly (i.e. a Free air anomaly plus the terrain correction $\left.\delta g_{T C}\right)$ and these gridded data can be seen in figure 8.1a.

Figure $8.1 \mathrm{~b}$ shows the gravity anomaly grid used to determine NZGeoid2009 and figure 8.2a shows the new gridded gravity data minus the gravity anomaly grid used to determine NZGeoid2009. It can be seen that the difference between the two grids largely consists of high frequency features which are present in the new gravity anomaly grid which were not present in the previously used grid.

Around shallow coastal areas and where the terrestrial gravity data's spatial density thins out the new gridded data also contains features which are not present in the previous grid. This is due to the airborne gravity data providing observations where there had previously been no data coverage due to the inaccessibility of these regions. These difference are shown in figure $8.2 \mathrm{~b}$. It is demonstrated in subsequent sections that these features improve the quality of the derived quasigeoid in comparison to the current quasigeoid NZGeoid2009. 

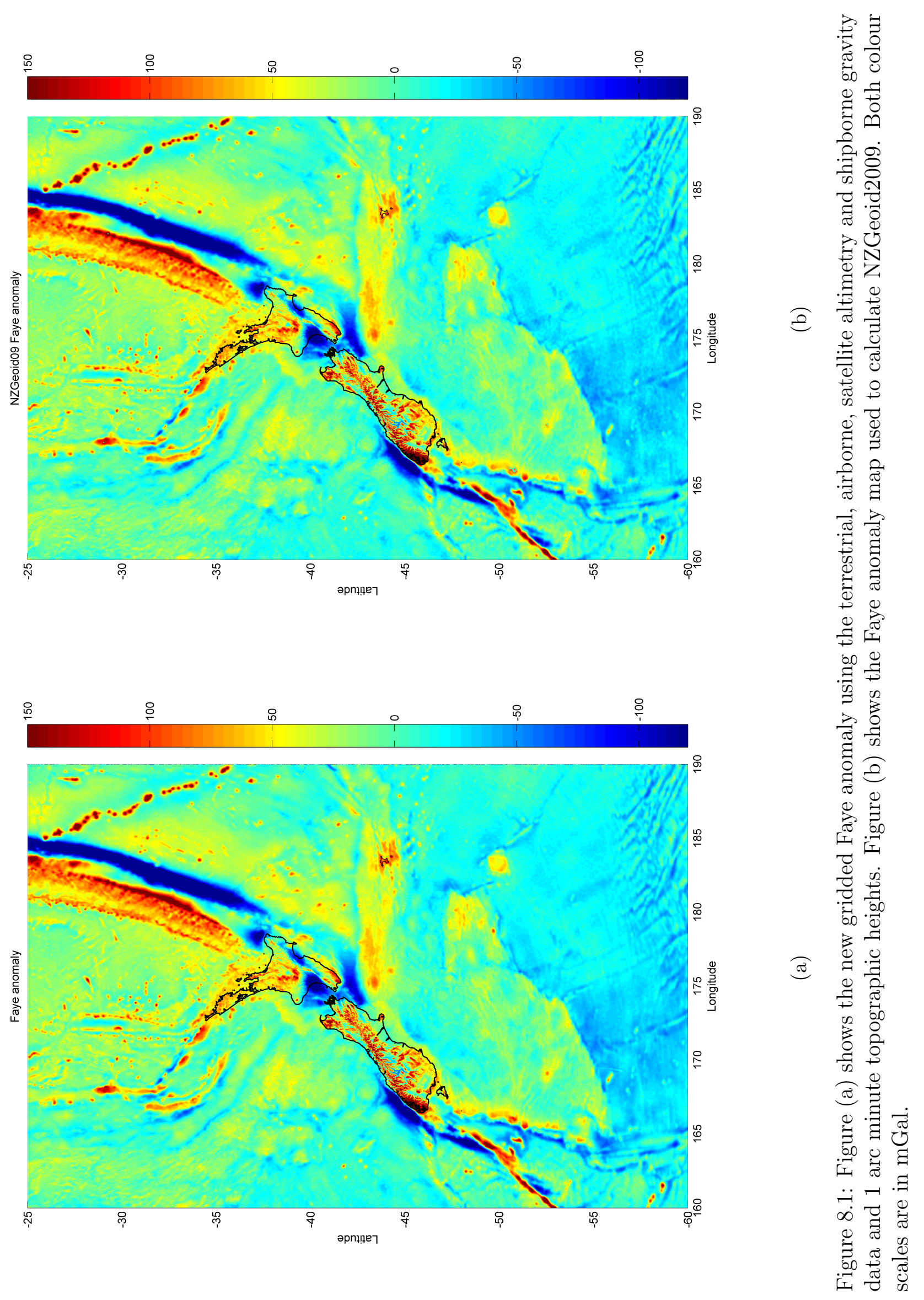

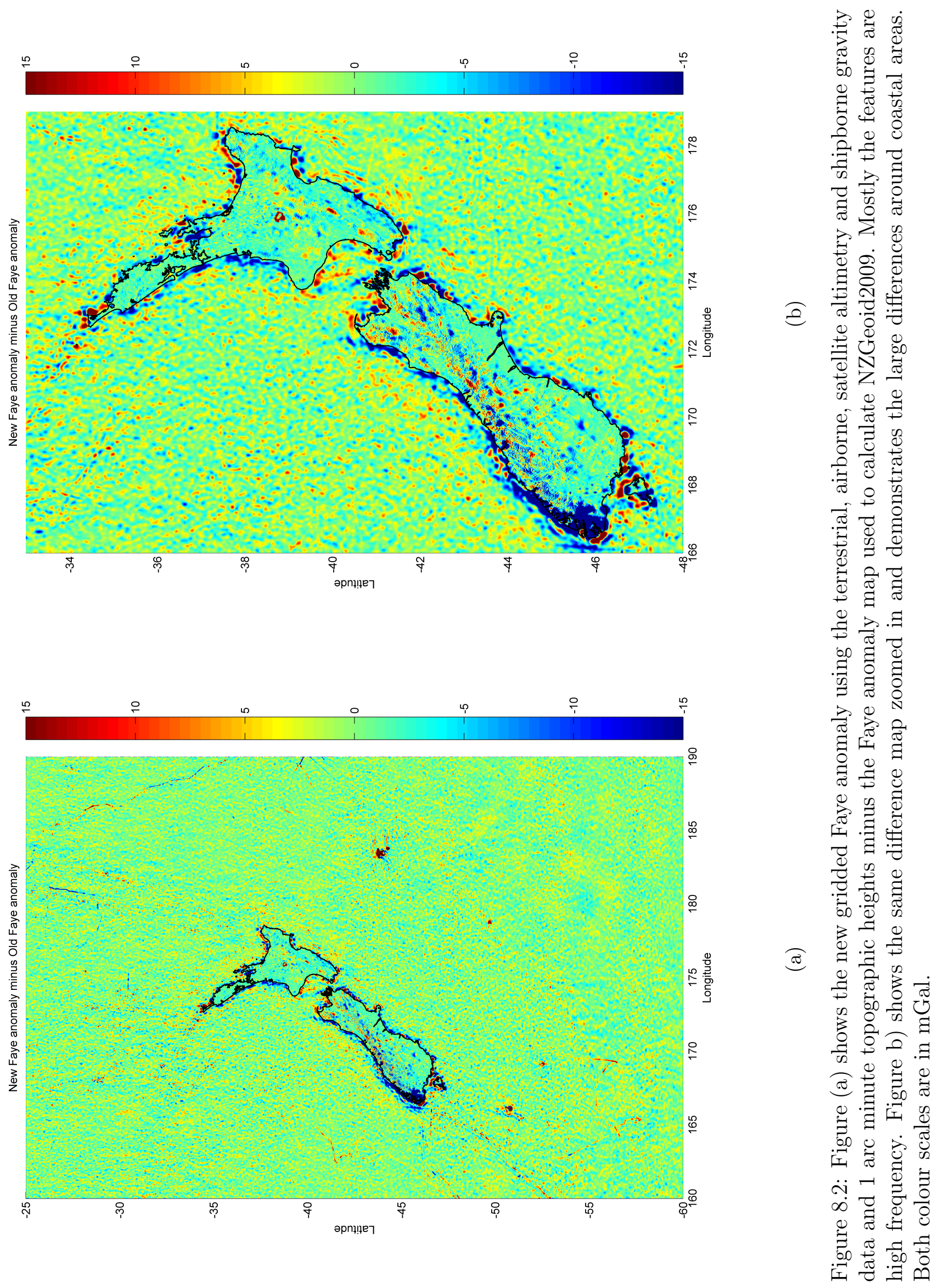


\subsection{Remove - Compute - Restore}

\subsubsection{Recap on the methodology of regional quasigeoid compu- tation}

In section 2.5 it was shown that to determine quasigeoid height anomalies, $N$, using Stokes integral, given here by equation 8.2 , requires the gravity data to be available over the entire Earth.

Gridded gravity data which only cover a small area of the Earth cannot be used to determine wavelengths longer than the region where the gravity data are available, i.e. the gravity signal is band limited. For example, it is easy to see that the mean Earth gravity value (i.e. the zero degree term in a spherical harmonic expansion) cannot be obtained from a small patch of gravity data. For this reason it was shown in section 2.5.2 that to compute a regional quasigeoid from gridded gravity data, the remove-compute-restore technique must be employed.

$$
N\left(\theta_{p}, \lambda_{p}\right)=\frac{R}{4 \pi \gamma} \int_{\psi} \Delta g(\theta, \lambda) S(\psi) \partial \psi
$$

The remove-compute-restore technique consists of subtracting a long wavelength gravity anomaly from a global gravity model to obtain a residual gravity anomaly, computing residual quasigeoid height anomalies from the residual gravity anomaly grid and then adding back the long wavelength quasigeoid height anomalies from the global gravity model.

Since the long wavelengths in the gravity field have been removed from the gridded gravity signal they must also be removed from the integral kernel $S(\psi)$ up to some maximum degree $L$ so that they are not resolved during the Stokes integration. Also since the low degree terms are removed from the kernel the integration only need to be performed inside a spherical cap denoted $\psi_{0}$. The Featherstone et al (1998) modified Stokes kernel, $S_{F}(\psi)$, which was determined in section 2.5.3 to be theoretically optimal, takes the following form,

$$
\hat{S}_{F}(\psi)=S(\psi)-\sum_{n=2}^{n=p} \frac{2 n+1}{n-1} P_{n}(\psi)-\sum_{k=2}^{k=L} \frac{2 k+1}{2} t_{k}\left(\psi_{0}\right) P_{k}(\psi) .
$$

and

$$
\begin{array}{lr}
S_{F}(\psi)=\hat{S}_{F}(\psi)-\hat{S}_{F}\left(\psi_{0}\right) & \text { for } 0 \leq \psi<\psi_{0} \\
S_{F}(\psi)=0 & \text { for } \psi_{0} \leq \psi \leq \pi .
\end{array}
$$

$t_{k}\left(\psi_{0}\right)$ are coefficients which are determined by finding the least squares solution to the following set of the linear equations

$$
\sum_{k=2}^{k=L} \frac{2 k+1}{2} t_{k}\left(\psi_{0}\right) e_{n k}(\psi)=Q_{n}\left(\psi_{0}\right)-\sum_{k=2}^{k=L} \frac{2 k+1}{2} e_{n k}(\psi)
$$

for $n=2,3,4,5, \ldots, L . Q_{n}\left(\psi_{0}\right)$ are given by equation 2.55 and $e_{n k}(\psi)$ are given by,

$$
e_{n k}(\psi)=\int_{\psi_{0}}^{\pi} P_{n}(\psi) P_{k}(\psi) \sin (\psi) d \psi
$$




\subsubsection{Computational steps taken to compute the new gravimet- ric quasigeoid}

Remove: The Eigen-6C4 global gravity model, expanded up to its maximum degree and order, was shown to have the best agreement with the leveling derived height anomalies in section 5.1. For this reason it has been used as the reference global gravity model for the remove compute restore procedure.

Figure $8.3 \mathrm{a}$ shows the Eigen-6C4 gridded global gravity model gravity anomaly at the gridded locations of the Faye anomaly shown in figure 7.1. Figure $8.3 \mathrm{~b}$ shows the residual gravity signal, i.e. the gridded Faye anomaly minus the Eigen-6C4 gravity anomaly.

Compute: Stokes integration was performed on the residual gravity grid using FFT1Dmod2009.f to obtain the residual quasigeoid height anomalies, $N_{\text {res }}$. This is a Fortran program which was used by Claessens et al (2009) to compute NZGeoid2009.

The program allows the user to specify the type of Stokes kernel modification, the degree of modification $L$ and the spherical cap $\psi_{0}$. The modification degree $L$ and spherical cap $\psi_{0}$ have been varied over the range $L=20,40, \ldots, 320$ and $\psi_{0}=1^{\circ}, 1.5^{\circ}, 2^{\circ}, 2.5^{\circ}, 3^{\circ}$ and the Stokes kernel modification type was chosen to be the Featherstone et al (1998) modified Stokes kernel.

Residual quasigeoid height anomalies have been computed for each combination of parameters. Each of the residual quasigeoid undulation grids took approximately 30 hours to process and 4 instances of the parameter sets were run in parallel using the c4.xlarge instance type of the Amazon cloud computing web services.

Restore: The long wavelength quasigeoid height anomalies of the global gravity model, $N_{L}$ were then calculated at the grid locations from the Eigen-6C4 global gravity model coefficients.

The primary indirect topographical effect, $N_{i n d}$, which is described in section 2.5, was also computed using equation 8.7 with heights, $H$, extracted from the 1 arc minute grid shown in figure 7.1.

$$
N_{\text {ind }}=\frac{-\pi G \rho H^{2}}{\gamma}
$$

Both the primary indirect topographical effect and the long wavelength quasigeoid height anomalies were then added back to the residual quasigeoid observations.

Figure 8.4a shows the Eigen-6C4 gridded global gravity model quasigeoid height anomalies at the gridded locations and figure $8.4 \mathrm{~b}$ shows the calculated primary indirect topographical effect.

Adding back these two grids to the residual quasigeoid height anomalies completes the restore step of the processing and so returns the final regional quasigeoid height anomalies $N$ over the computation area, where

$$
N=N_{\text {res }}+N_{L}-N_{\text {ind }} .
$$




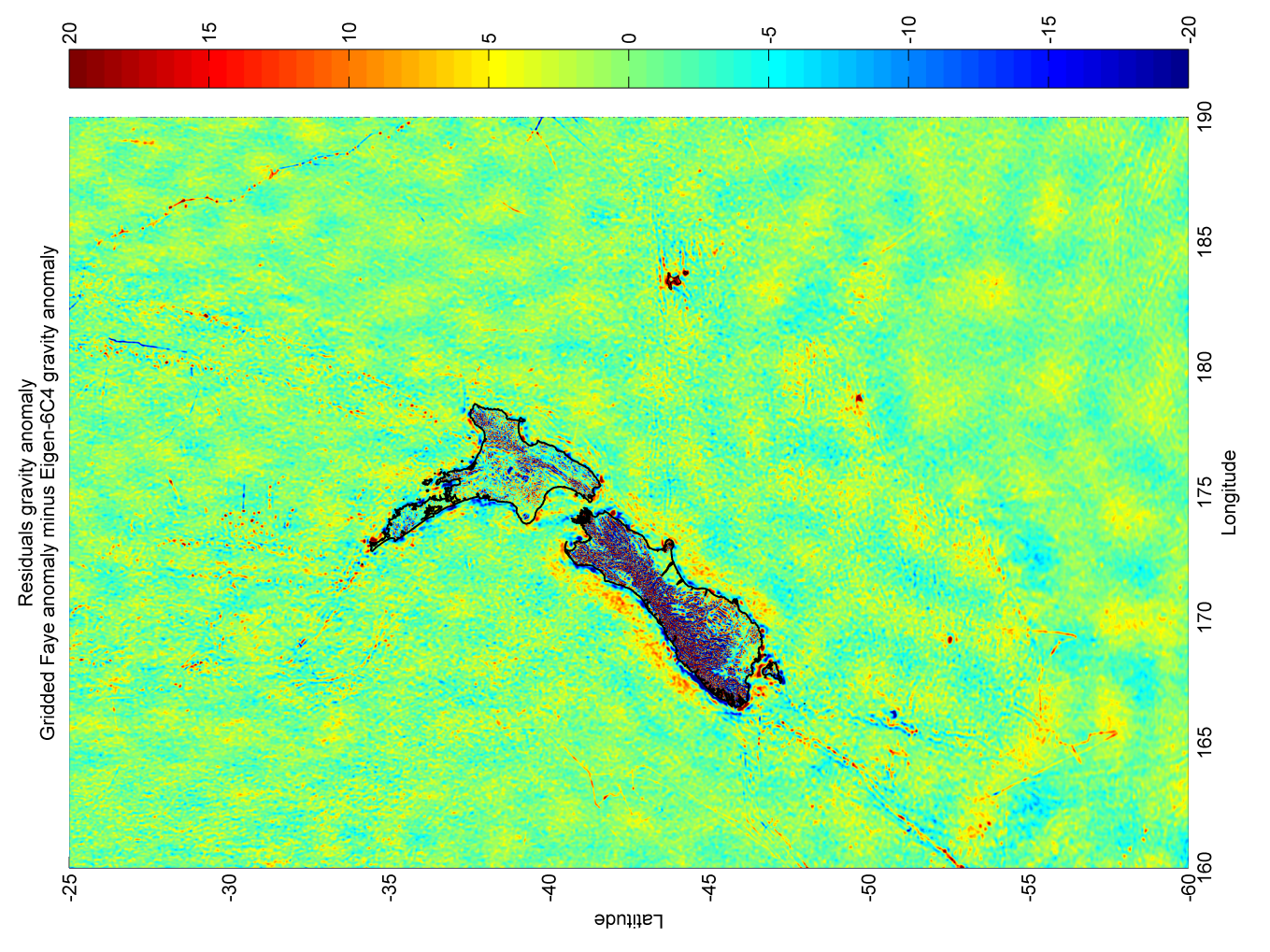

0
$\vdots$
0
$\frac{0}{4}$
0
0
0
0
0
0
01

完

อ $\frac{\pi}{0}$

吾

ปี

䒕

응

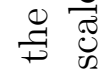

*

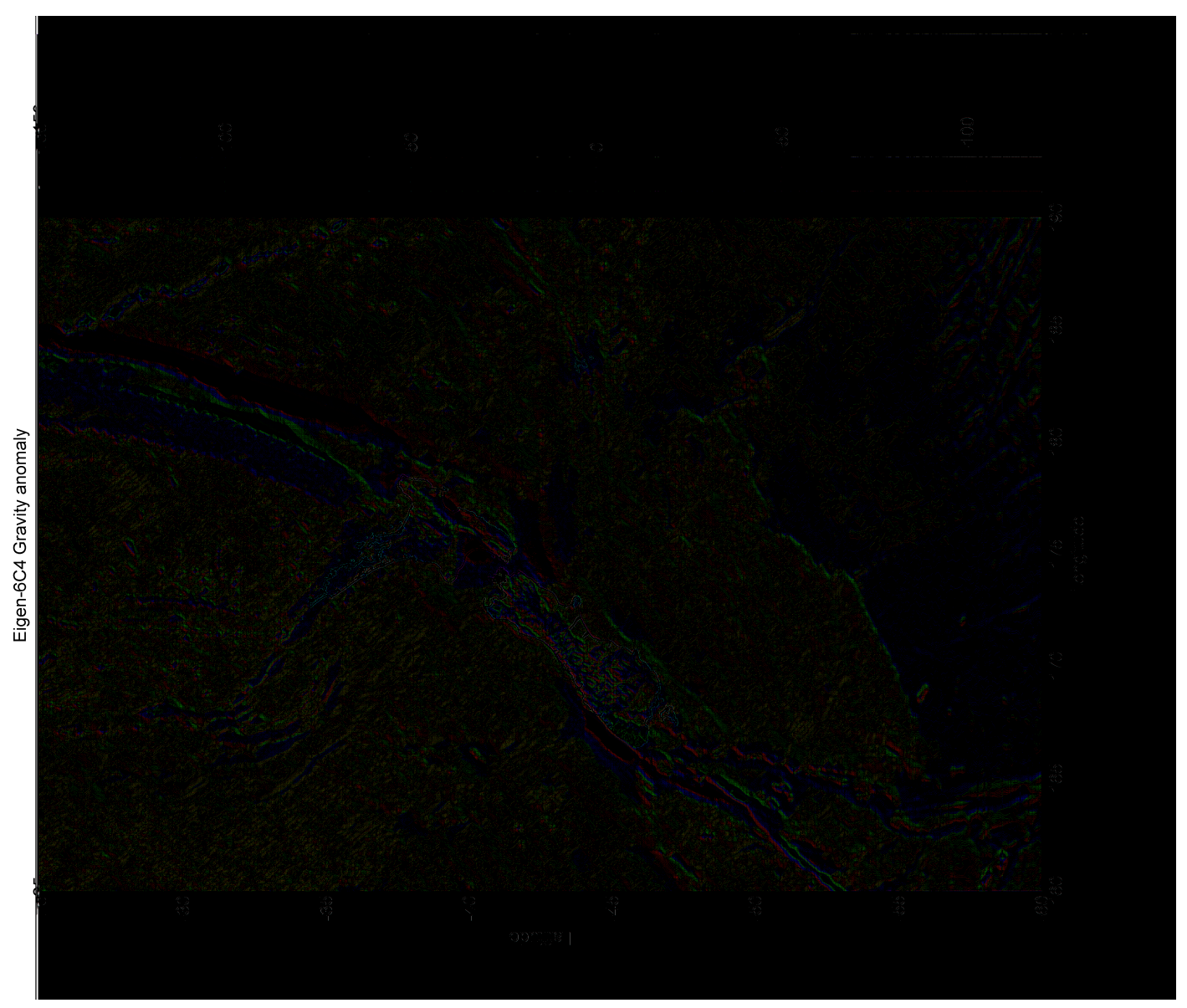

吕

莺

कू

तె

E

表离

50

5

क्ष 0

से

00

I诖

80

๙

秀

궁

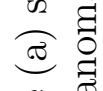

节

江 不

$\ddot{\infty}$ 总

.

.08 

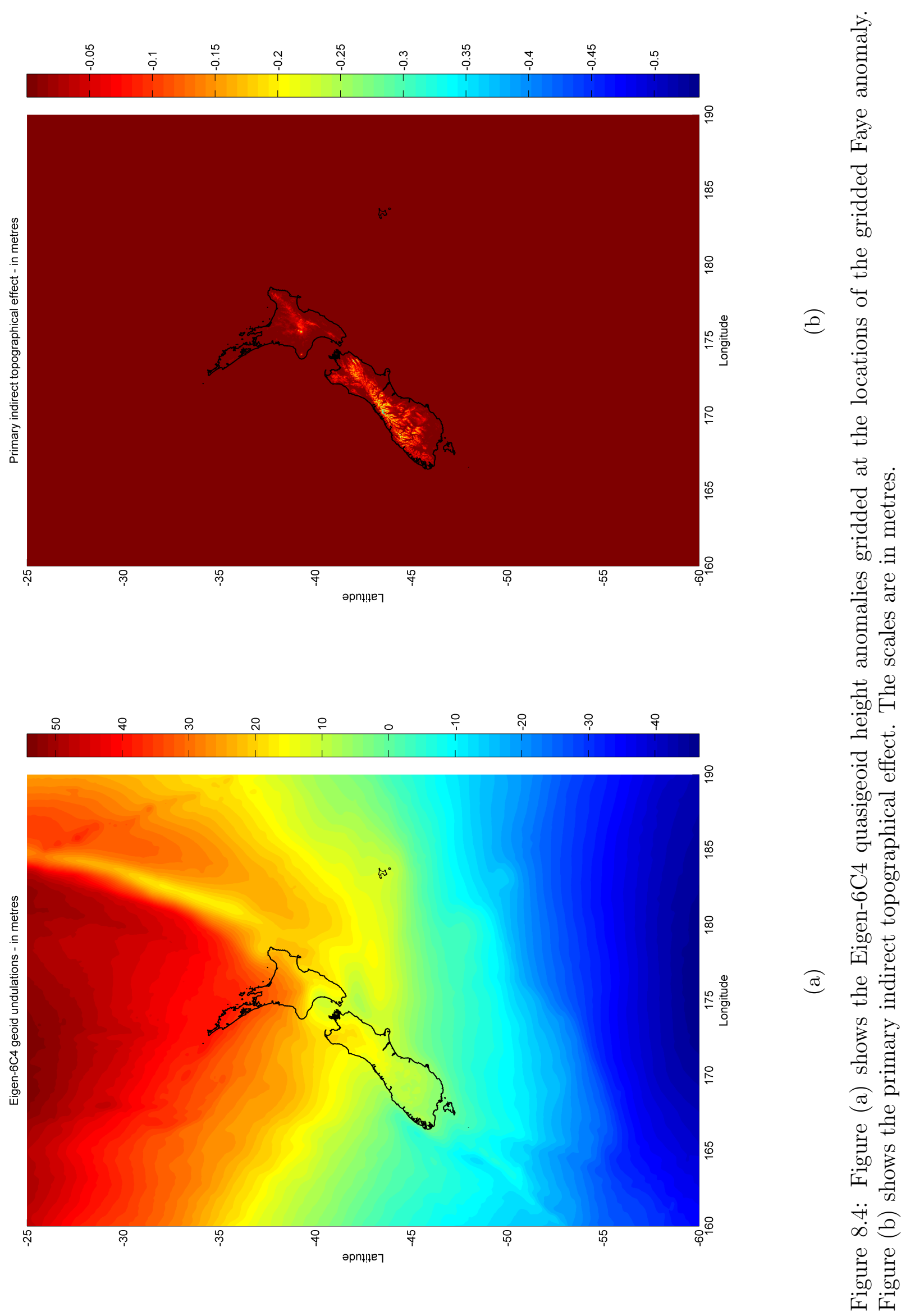


\subsection{Results}

For each parameter variation of $L$ and $\psi_{0}$ the gridded geoids have been compared to the leveling derived height anomalies. As was similarly done for NZGeoid2009, a mean has been computed for each local vertical datum and subtracted from the differences, following section 3.4 . Tables 8.1 and 8.2 show the standard deviation (estimated from the inner $68 \%$ of the distribution) and root mean squared of the differences for each parameter combination.

Table 8.1: Standard deviation estimated from the inner $68 \%$ of the distribution of leveling derived height anomalies minus the quasigeoid height anomalies derived with parameter combinations $L$ and $\psi_{0}$. The units are centimetres.

\begin{tabular}{|l|l|l|l|l|l|l|l|l|}
\hline & $\mathbf{L = 2 0}$ & $\mathbf{L}=\mathbf{4 0}$ & $\mathbf{L = 6 0}$ & $\mathbf{L}=\mathbf{8 0}$ & $\mathbf{L}=\mathbf{1 0 0}$ & $\mathbf{L}=\mathbf{1 2 0}$ & $\mathbf{L}=\mathbf{1 4 0}$ & $\mathbf{L}=\mathbf{1 6 0}$ \\
\hline$\psi_{0}=\mathbf{1}^{\circ}$ & 4.670 & 4.658 & 4.633 & 4.598 & 4.555 & 4.514 & 4.468 & 4.423 \\
\hline$\psi_{0}=\mathbf{1 . 5}^{\circ}$ & 5.039 & 5.008 & 4.961 & 4.899 & 4.831 & 4.768 & 4.701 & 4.628 \\
\hline$\psi_{0}=\mathbf{2}^{\circ}$ & 5.102 & 5.072 & 5.026 & 4.970 & 4.912 & 4.843 & 4.735 & 4.559 \\
\hline$\psi_{0}=\mathbf{2 . 5 ^ { \circ }}$ & 5.060 & 5.040 & 5.006 & 4.967 & 4.910 & 4.791 & 4.599 & 4.420 \\
\hline$\psi_{0}=\mathbf{3}^{\circ}$ & 5.044 & 5.029 & 5.004 & 4.967 & 4.877 & 4.743 & 4.619 & 4.510 \\
\hline & $\mathbf{L = 1 8 0}$ & $\mathbf{L = 2 0 0}$ & $\mathbf{L = 2 2 0}$ & $\mathbf{L}=\mathbf{2 4 0}$ & $\mathbf{L}=\mathbf{2 6 0}$ & $\mathbf{L}=\mathbf{2 8 0}$ & $\mathbf{L}=\mathbf{3 0 0}$ & $\mathbf{L}=\mathbf{3 2 0}$ \\
\hline$\psi_{0}=\mathbf{1}^{\circ}$ & 4.379 & 4.342 & 4.301 & 4.261 & 4.228 & 4.187 & 4.154 & 4.118 \\
\hline$\psi_{0}=\mathbf{1 . 5}^{\circ}$ & 4.548 & 4.431 & 4.263 & 4.072 & 3.897 & 3.833 & 3.858 & 3.935 \\
\hline$\psi_{0}=\mathbf{2}^{\circ}$ & 4.324 & 4.138 & 4.039 & 3.981 & 3.955 & 3.992 & 4.437 & 13.712 \\
\hline$\psi_{0}=\mathbf{2 . 5 ^ { \circ }}$ & 4.304 & 4.219 & 4.156 & 4.138 & 4.389 & 32.597 & 7.436 & 6.811 \\
\hline$\psi_{0}=\mathbf{3}^{\circ}$ & 4.420 & 4.337 & 4.246 & 4.091 & 3.871 & 3.834 & 3.843 & 3.912 \\
\hline
\end{tabular}

Table 8.2: Root mean squared of the distribution of leveling derived height anomalies minus the quasigeoid height anomalies derived with parameter combinations $L$ and $\psi_{0}$. The units are centimetres.

\begin{tabular}{|l|l|l|l|l|l|l|l|l|}
\hline & $\mathbf{L}=\mathbf{2 0}$ & $\mathbf{L}=\mathbf{4 0}$ & $\mathbf{L}=\mathbf{6 0}$ & $\mathbf{L}=\mathbf{8 0}$ & $\mathbf{L}=\mathbf{1 0 0}$ & $\mathbf{L}=\mathbf{1 2 0}$ & $\mathbf{L}=\mathbf{1 4 0}$ & $\mathbf{L}=\mathbf{1 6 0}$ \\
\hline$\psi_{0}=\mathbf{1}^{\circ}$ & 5.757 & 5.740 & 5.710 & 5.671 & 5.621 & 5.569 & 5.515 & 5.463 \\
\hline$\psi_{0}=\mathbf{1 . 5}^{\circ}$ & 6.170 & 6.160 & 6.144 & 6.110 & 6.013 & 5.841 & 5.680 & 5.555 \\
\hline$\psi_{0}=\mathbf{2}^{\circ}$ & 6.273 & 6.238 & 6.187 & 6.125 & 6.054 & 5.970 & 5.842 & 5.628 \\
\hline$\psi_{0}=\mathbf{2 . 5}$ & 6.194 & 6.178 & 6.151 & 6.114 & 6.050 & 5.912 & 5.671 & 5.450 \\
\hline$\psi_{0}=\mathbf{3}^{\circ}$ & 6.217 & 6.182 & 6.122 & 6.049 & 5.966 & 5.888 & 5.806 & 5.718 \\
\hline & $\mathbf{L = 1 8 0}$ & $\mathbf{L = 2 0 0}$ & $\mathbf{L = 2 2 0}$ & $\mathbf{L = 2 4 0}$ & $\mathbf{L}=\mathbf{2 6 0}$ & $\mathbf{L}=\mathbf{2 8 0}$ & $\mathbf{L}=\mathbf{3 0 0}$ & $\mathbf{L}=\mathbf{3 2 0}$ \\
\hline$\psi_{0}=\mathbf{1}^{\circ}$ & 5.414 & 5.366 & 5.321 & 5.280 & 5.239 & 5.196 & 5.157 & 5.111 \\
\hline$\psi_{0}=\mathbf{1 . 5}^{\circ}$ & 5.445 & 5.345 & 5.236 & 5.062 & 4.861 & 4.819 & 4.823 & 4.821 \\
\hline$\psi_{0}=\mathbf{2}^{\circ}$ & 5.350 & 5.134 & 5.017 & 4.961 & 4.940 & 4.970 & 5.436 & 15.240 \\
\hline$\psi_{0}=\mathbf{2 . 5}^{\circ}$ & 5.303 & 5.204 & 5.134 & 5.116 & 5.400 & 39.049 & 7.622 & 6.941 \\
\hline$\psi_{0}=\mathbf{3}^{\circ}$ & 5.615 & 5.474 & 5.279 & 5.059 & 4.898 & 4.844 & 4.852 & 4.882 \\
\hline
\end{tabular}

Figure 8.5 and 8.6 show the standard deviation of the inner $68 \%$ and the root mean squared of the differences, respectively, for each spherical cap $\psi_{0}$ plotted against the modification degree $L$. 


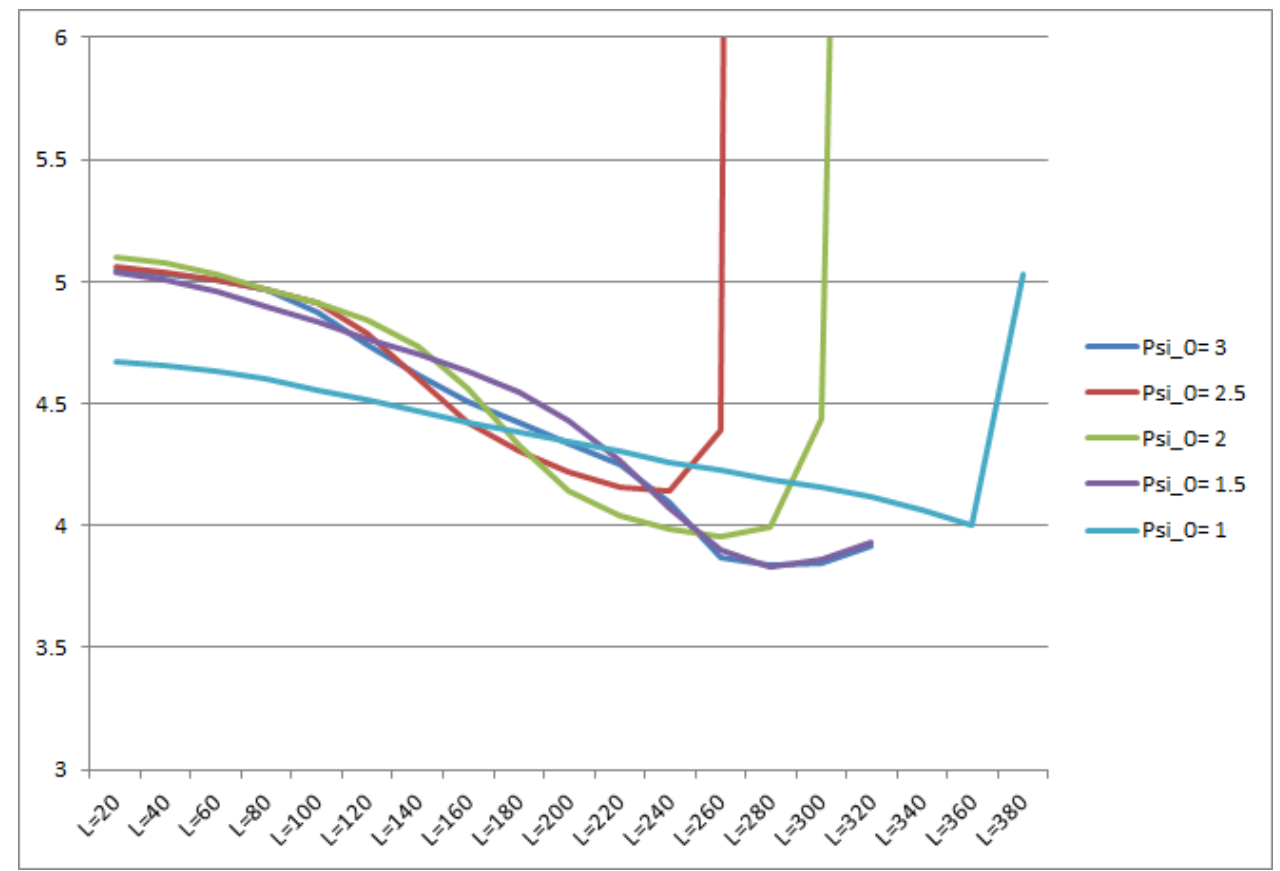

Figure 8.5: Standard deviation estimated from the inner $68 \%$ of the leveling height anomalies minus the quasigeoid computed with parameters $\psi_{0}$ and $L$.

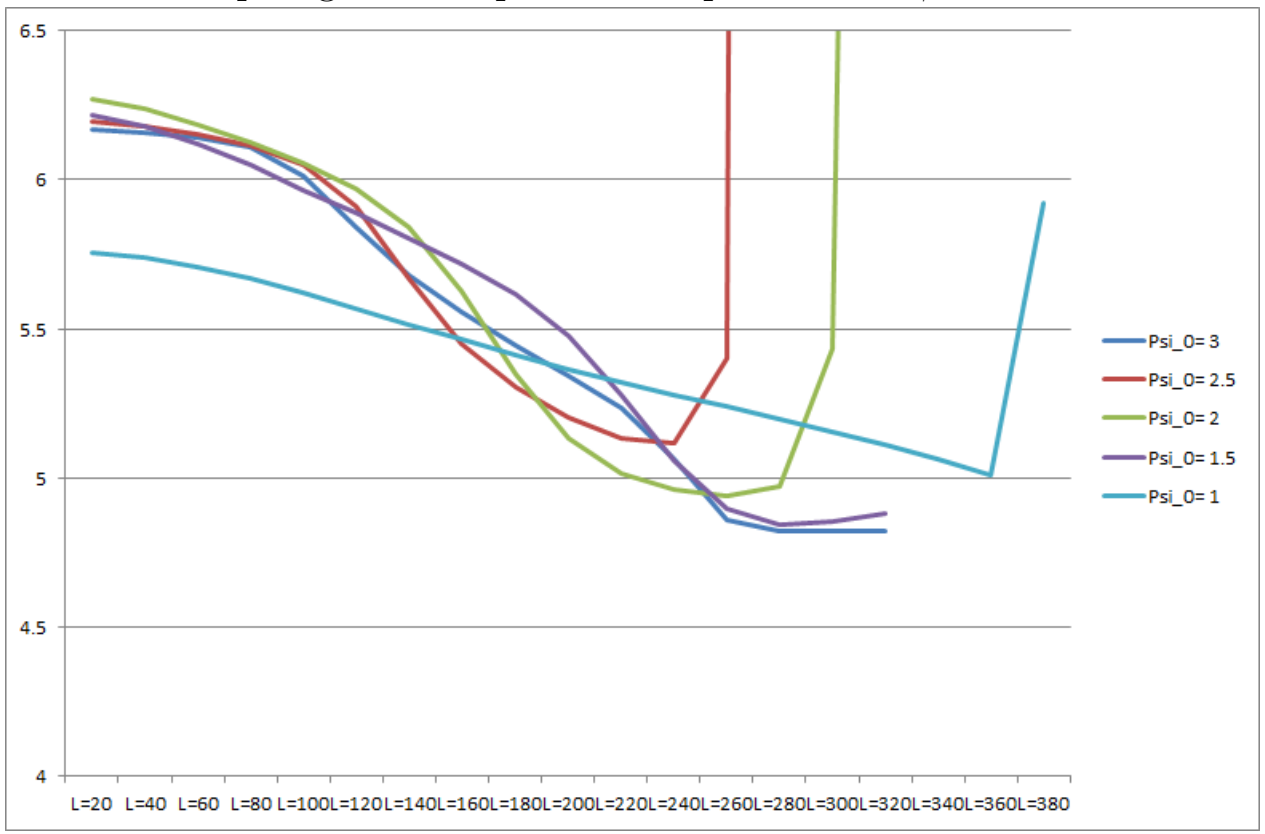

Figure 8.6: Root mean squared of the leveling height anomalies minus the quasigeoid computed with parameters $\psi_{0}$ and $L$. 
As the $L$ parameter increases more wavelengths are removed from the integration kernel and so the residual gridded gravity anomaly is used to determine smaller maximum wavelengths of the geoid undulations. From figures 8.5 and 8.6 it can be seen that:

- For spherical caps $\psi_{0}=1.5,2 \& 2.5$ the standard deviation and root mean squared smoothly decay until reaching a minimum and then increase, the smallest standard deviation and root mean squared is given by $\psi_{0}=1.5$ where $L=280$.

- For the spherical cap $\psi_{0}=3$ the decay of the standard deviation and root mean squared is not as smooth as for $\psi_{0}=1.5,2 \& 2.5$ and it reaches a negligibly different minimum to that of $\psi_{0}=1.5$.

- For the spherical cap $\psi_{0}=1$ there is a significantly shallower decay as $L$ increases than for all the other tested values of $\psi_{0}$, and a sharp turning point between $L=360$ and $L=380$. This could be attributable to the spherical cap not being wide enough to evaluate the longer wavelengths in the quasigeoid height anomalies, i.e. taking $\psi_{0}=1$ could be cutting too much off of the ends of the modified Stokes kernel $S_{F}(\psi)$, where the ends are not sufficiently close to zero, causing aliasing.

Spherical caps $\psi_{0}=1.5,2 \& 2.5$ have a minima at $L=280,260 \& 240$, respectively. The minimum standard deviation for each of these parameter combinations are $3.8,3.9 \& 4.1 \mathrm{~cm}$ and 4.8 , $4.9 \& 5.1 \mathrm{~cm}$ for the root mean squared residual. There is little difference between them and so the quasigeoid overall accuracy is fairly insensitive for these choices of parameter combinations, although overall $\psi_{0}=1.5$ and $L=280$ gives the best fit. Similarly, for $\psi_{0}=3$ the minimum is at $L=280$ with the a standard deviation of $3.834 \mathrm{~cm}$. However this is less than $10^{-2} \mathrm{~cm}$ different from that of $\psi_{0}=1.5$ and $L=280$, again indicating the insensitivity of the parameter choice.

For the reasons stated above it appears that the preferable parameter choice is $\psi_{0}=1.5$ and $L=280$. Figures $8.7 \mathrm{a}$ and $8.7 \mathrm{~b}$ show the residual geoid undulations for this parameter combination and the resulting quasigeoid after restoring the global gravity model and topographic effect. Figure 8.8a shows the leveling derived height anomalies minus the quasigeoid computed with parameters $\psi_{0}=1.5$ and $L=280$ after subtracting mean local vertical datum offsets, for reference figure 8.8b shows the same thing for NZGeoid2009. It can be seen in these figures that overall the new quasigeoid fits the leveling derived heights anomalies better than NZGeoid2009, in particular over coastal areas and in rough topography through the centre of the South Island. This is due to the contribution of the new airborne gravity data where previously there was poor coverage. The new quasigeoid model out-performs both the current official quasigeoid NZGeoid2009 and the global gravity model Eigen-6C4 in terms of its fit to the leveling derived height anomalies. This is evidenced in table 8.3 which gives the statistics of the differences.

Table 8.3: Statistics of the difference between leveling derived height anomalies, and those of the $\psi_{0}=1.5$ and $L=280$ quasigeoid, NZGeoid2009 and Eigen-6C4. Values are in cm.

$\begin{array}{lllll}\text { Std } & \text { RMS } & \text { Kurtosis } & \text { Minimum } & \text { Maximum } \\ 3.83 & 4.81 & 6.03 & -27.62 & 26.11 \\ 4.88 & 6.14 & 6.76 & -37.69 & 28.3 \\ 4.57 & 5.29 & 5.96 & -32.93 & 29.05\end{array}$



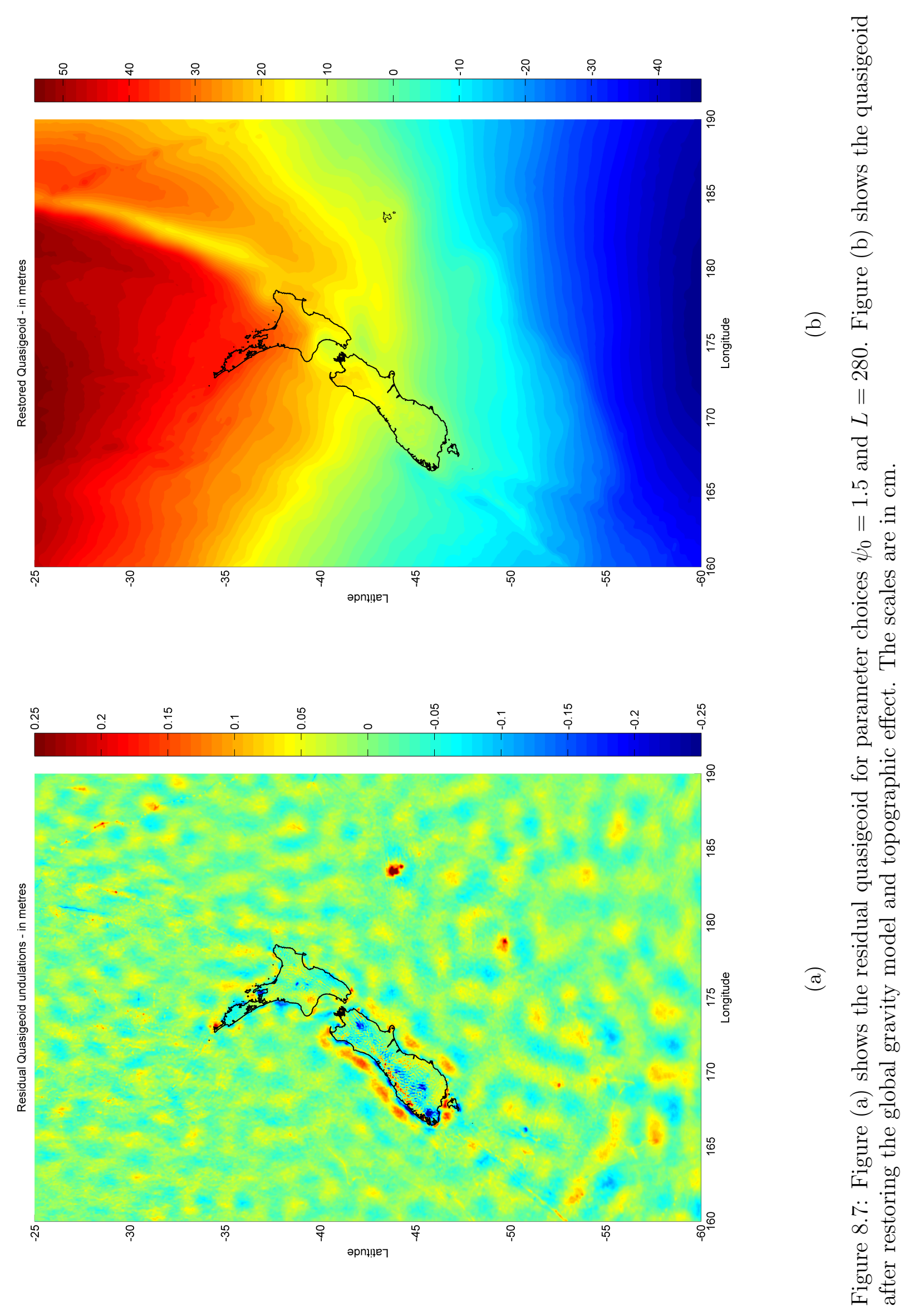


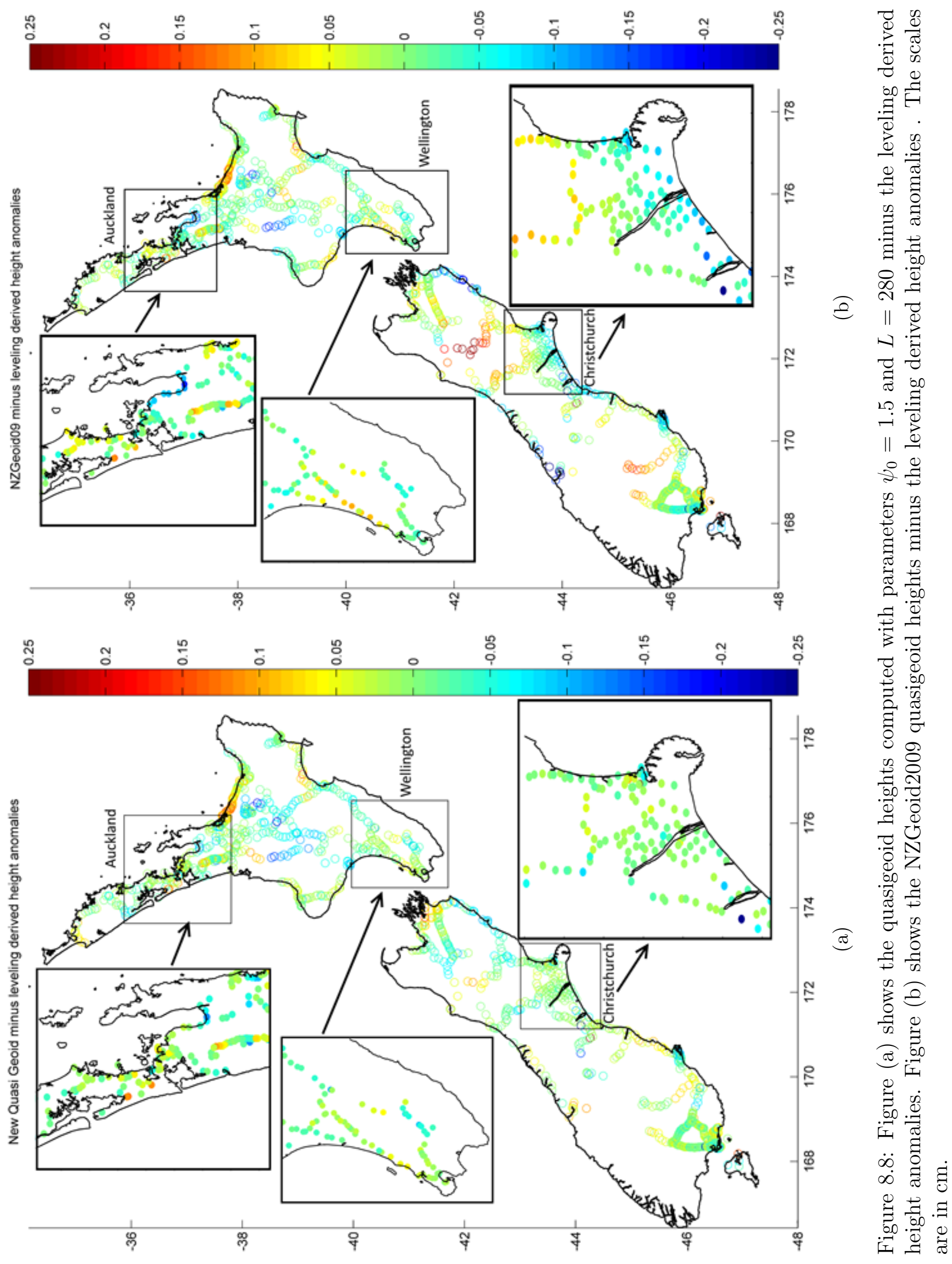


The accuracy of the $\psi_{0}=1.5$ and $L=280$ quasigeoid has also been evaluated in 3 main urban areas, Auckland, Wellington and Christchurch. These areas are shown in figures 8.8a and 8.8b. The accuracy in these densely populated urban regions is of particular importance for engineering purposes. For these urban areas, tables 8.4 and 8.5 shows the standard deviation estimated from the inner $68 \%$ and the root mean squared of the differences between the leveling derived height anomalies and the $\psi_{0}=1.5$ and $L=280$ quasigeoid, and similarly for NZGeoid2009.

Table 8.4: Standard deviation of the inner $68 \%$ of the differences between the leveling derived height anomalies and the $\left(\psi_{0}=1.5, L=280\right)$ quasigeoid, and NZGeoid2009

$\begin{array}{llll}\text { Standard deviation } & \text { Auckland } & \text { Wellington } & \text { Christchurch } \\ \text { Quasigeoid } \psi_{0}=1.5 \text { and } L=280 & 3.976 & 3.385 & 2.071 \\ \text { NZGeoid2009 } & 3.427 & 5.158 & 5.063 \\ \text { Number of points } & 123 & 169 & 125\end{array}$

Table 8.5: Root mean squared of the differences between the leveling derived height anomalies and the $\left(\psi_{0}=1.5, L=280\right)$ quasigeoid, and NZGeoid2009

$\begin{array}{llll}\text { RMS } & \text { Auckland } & \text { Wellington } & \text { Christchurch } \\ \text { Quasigeoid } \psi_{0}=1.5 \text { and } L=280 & 3.580 & 4.388 & 4.572 \\ \text { NZGeoid2009 } & 3.733 & 5.127 & 6.164 \\ \text { Number of points } & 123 & 169 & 125\end{array}$

It can again be seen here that the $\psi_{0}=1.5$ and $L=280$ quasigeoid outperforms the NZGeoid2009 in terms of fit to the leveling derived height anomalies. In these areas the $\psi_{0}=1.5$ and $L=280$ quasigeoid has a mean standard deviation of $3.1 \mathrm{~cm}$ which is more than $1 \mathrm{~cm}$ better than the mean standard deviation of $4.68 \mathrm{~cm}$ for NZGeoid2009.

\subsubsection{Additional leveling/GPS points}

Up until here, the leveling derived height anomalies have come from a database which was used to evaluate the accuracy NZGeoid2009. It consists of joint leveling and GPS observations at 1442 points in 13 local vertical datums. Each datum has a mean offset from the quasigeoid which has been computed from the $\psi_{0}=1.5$ and $L=280$ quasigeoid and NZGeoid2009. These offsets are shown table 8.6. Differences between the two sets of datum offsets are attributable to differences in long wavelength quasigeoid height anomalies between the two models. The two sets of offsets are highly correlated but the new offsets are smaller which indicates a better initial fit of the quasigeoid to the leveling-derived height anomalies.

Since this original database was put together another 1454 leveling points have been located using GPS which have been added to the database. The fit of the quasigeoid for each parameter combination $L$ and $\psi_{0}$ to the new set of leveling derived height anomalies has been computed in terms of the standard deviation and root mean squared, as before.

After recomputing mean local vertical datum offsets the new differences appear to contain significantly more outliers than before. This is evidenced in the large difference between the RMS of $14.77 \mathrm{~cm}$ and standard deviation of $5.44 \mathrm{~cm}$ for the NZGeoid2009 differences, and by the large difference between the root mean squared and standard deviations in tables 8.7 and 8.8 for the various $L$ and $\psi_{0}$ quasigeoids. 
Table 8.6: Local vertical datum offsets given for NZGeoid2009 and computed for the original leveling data. Values are given in $\mathrm{cm}$.

\begin{tabular}{|l|l|l|}
\hline LVD & NZGeoid2009 Offset in $\mathbf{~ c m}$ & Quasigeoid $\psi_{0}=1.5$ and $L=280$ in $\mathbf{~ c m}$ \\
\hline Auckland & 33.90 & 25.48 \\
\hline Bluff & 36.00 & 22.75 \\
\hline Dun/Bluff & 38.10 & 19.41 \\
\hline Dunedin & 48.50 & 30.13 \\
\hline Gisbourne & 34.40 & 29.83 \\
\hline Lyttleton & 46.60 & 29.23 \\
\hline Moturiki & 24.10 & 21.69 \\
\hline Napier & 20.30 & 17.63 \\
\hline Nelson & 29.40 & 26.46 \\
\hline One tree point & 6.30 & 2.77 \\
\hline Stewart Island & 38.50 & 22.70 \\
\hline Taranaki & 31.50 & 25.41 \\
\hline Wellington & 43.60 & 31.99 \\
\hline
\end{tabular}

Table 8.7: Std. dev. of inner $68 \%$ of 2857 leveling height anomalies minus quasigeoid for $\psi_{0}$ and $L$.

\begin{tabular}{|l|l|l|l|l|l|l|l|l|}
\hline Std. dev. & $\mathbf{L = 2 0}$ & $\mathbf{L}=\mathbf{4 0}$ & $\mathbf{L}=\mathbf{6 0}$ & $\mathbf{L}=\mathbf{8 0}$ & $\mathbf{L}=\mathbf{1 0 0}$ & $\mathbf{L}=\mathbf{1 2 0}$ & $\mathbf{L}=\mathbf{1 4 0}$ & $\mathbf{L}=\mathbf{1 6 0}$ \\
\hline$\psi_{0}=\mathbf{1}^{\circ}$ & 5.263 & 5.248 & 5.216 & 5.173 & 5.120 & 5.059 & 4.997 & 4.935 \\
\hline$\psi_{0}=\mathbf{1 . 5}^{\circ}$ & 5.530 & 5.533 & 5.531 & 5.525 & 5.496 & 5.416 & 5.298 & 5.168 \\
\hline$\psi_{0}=\mathbf{2}^{\circ}$ & 5.672 & 5.650 & 5.614 & 5.570 & 5.518 & 5.453 & 5.348 & 5.147 \\
\hline$\psi_{0}=\mathbf{2 . 5 ^ { \circ }}$ & 5.554 & 5.552 & 5.545 & 5.532 & 5.502 & 5.420 & 5.243 & 5.027 \\
\hline$\psi_{0}=\mathbf{3}^{\circ}$ & 5.709 & 5.676 & 5.621 & 5.558 & 5.482 & 5.403 & 5.323 & 5.231 \\
\hline & $\mathbf{L = 1 8 0}$ & $\mathbf{L = 2 0 0}$ & $\mathbf{L = 2 2 0}$ & $\mathbf{L = 2 4 0}$ & $\mathbf{L = 2 6 0}$ & $\mathbf{L = 2 8 0}$ & $\mathbf{L}=\mathbf{3 0 0}$ & $\mathbf{L}=\mathbf{3 2 0}$ \\
\hline$\psi_{0}=\mathbf{1}^{\circ}$ & 4.874 & 4.818 & 4.762 & 4.709 & 4.653 & 4.594 & 4.534 & 4.464 \\
\hline$\psi_{0}=\mathbf{1 . 5}^{\circ}$ & 5.036 & 4.904 & 4.747 & 4.472 & 4.077 & 3.949 & 3.975 & 3.996 \\
\hline$\psi_{0}=\mathbf{2}^{\circ}$ & 4.840 & 4.561 & 4.378 & 4.279 & 4.233 & 4.296 & 5.015 & 14.774 \\
\hline$\psi_{0}=\mathbf{2 . 5}$ & 4.844 & 4.702 & 4.596 & 4.567 & 4.996 & 35.155 & 7.161 & 6.410 \\
\hline$\psi_{0}=\mathbf{3}^{\circ}$ & 5.117 & 4.950 & 4.709 & 4.395 & 4.105 & 3.976 & 3.947 & 3.971 \\
\hline
\end{tabular}

Table 8.8: RMS of 2857 leveling height anomalies minus quasigeoid for $\psi_{0}$ and $L$.

\begin{tabular}{|l|l|l|l|l|l|l|l|l|}
\hline RMS & $\mathbf{L = 2 0}$ & $\mathbf{L}=\mathbf{4 0}$ & $\mathbf{L}=\mathbf{6 0}$ & $\mathbf{L}=\mathbf{8 0}$ & $\mathbf{L}=\mathbf{1 0 0}$ & $\mathbf{L}=\mathbf{1 2 0}$ & $\mathbf{L}=\mathbf{1 4 0}$ & $\mathbf{L}=\mathbf{1 6 0}$ \\
\hline$\psi_{0}=\mathbf{1}^{\circ}$ & 14.593 & 14.588 & 14.576 & 14.560 & 14.543 & 14.525 & 14.505 & 14.487 \\
\hline$\psi_{0}=\mathbf{1 . 5}^{\circ}$ & 14.769 & 14.764 & 14.759 & 14.747 & 14.710 & 14.644 & 14.582 & 14.533 \\
\hline$\psi_{0}=\mathbf{2}^{\circ}$ & 14.819 & 14.804 & 14.782 & 14.756 & 14.727 & 14.692 & 14.639 & 14.554 \\
\hline$\psi_{0}=\mathbf{2 . 5}$ & 14.775 & 14.770 & 14.761 & 14.748 & 14.723 & 14.670 & 14.578 & 14.493 \\
\hline$\psi_{0}=\mathbf{3}^{\circ}$ & 14.805 & 14.790 & 14.762 & 14.730 & 14.694 & 14.660 & 14.625 & 14.590 \\
\hline & $\mathbf{L = 1 8 0}$ & $\mathbf{L = 2 0 0}$ & $\mathbf{L = 2 2 0}$ & $\mathbf{L}=\mathbf{2 4 0}$ & $\mathbf{L}=\mathbf{2 6 0}$ & $\mathbf{L}=\mathbf{2 8 0}$ & $\mathbf{L}=\mathbf{3 0 0}$ & $\mathbf{L}=\mathbf{3 2 0}$ \\
\hline$\psi_{0}=\mathbf{1}^{\circ}$ & 14.470 & 14.454 & 14.439 & 14.426 & 14.414 & 14.401 & 14.389 & 14.377 \\
\hline$\psi_{0}=\mathbf{1 . 5}^{\circ}$ & 14.491 & 14.454 & 14.414 & 14.356 & 14.301 & 14.301 & 14.297 & 14.285 \\
\hline$\psi_{0}=\mathbf{2}^{\circ}$ & 14.451 & 14.376 & 14.340 & 14.325 & 14.320 & 14.327 & 14.470 & 21.356 \\
\hline$\psi_{0}=\mathbf{2 . 5}$ & 14.438 & 14.403 & 14.378 & 14.373 & 14.473 & 44.125 & 15.859 & 15.457 \\
\hline$\psi_{0}=\mathbf{3}^{\circ}$ & 14.549 & 14.495 & 14.426 & 14.355 & 14.316 & 14.316 & 14.335 & 14.359 \\
\hline
\end{tabular}




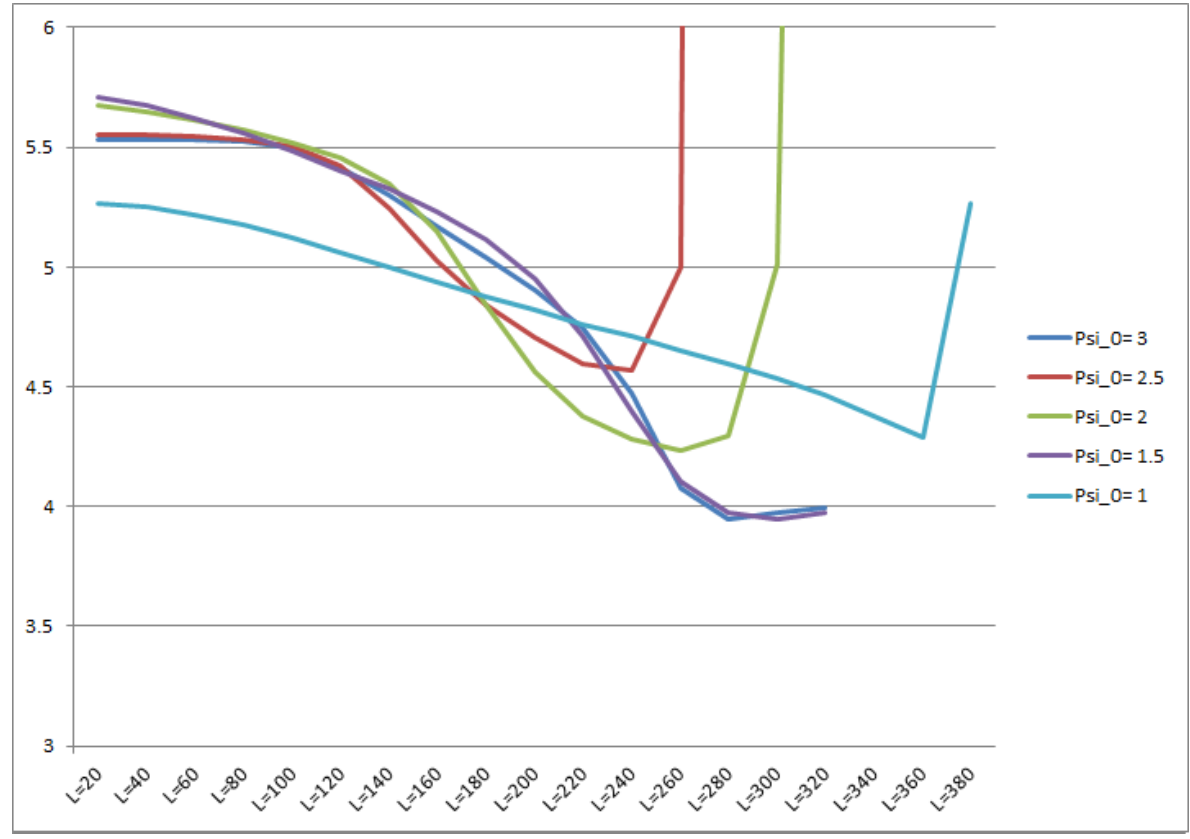

Figure 8.9: Standard deviation of the inner $68 \%$ of the leveling height anomalies minus the quasigeoid computed with parameters $\psi_{0}$ and $L$. Scale is in $\mathrm{cm}$

Figure 8.9 and 8.10 show the standard deviation estimated from the inner $68 \%$ and the root mean squared of the differences, respectively, for each spherical cap $\psi_{0}$ plotted against the modification degree $L$ for the larger leveling height anomaly data base.

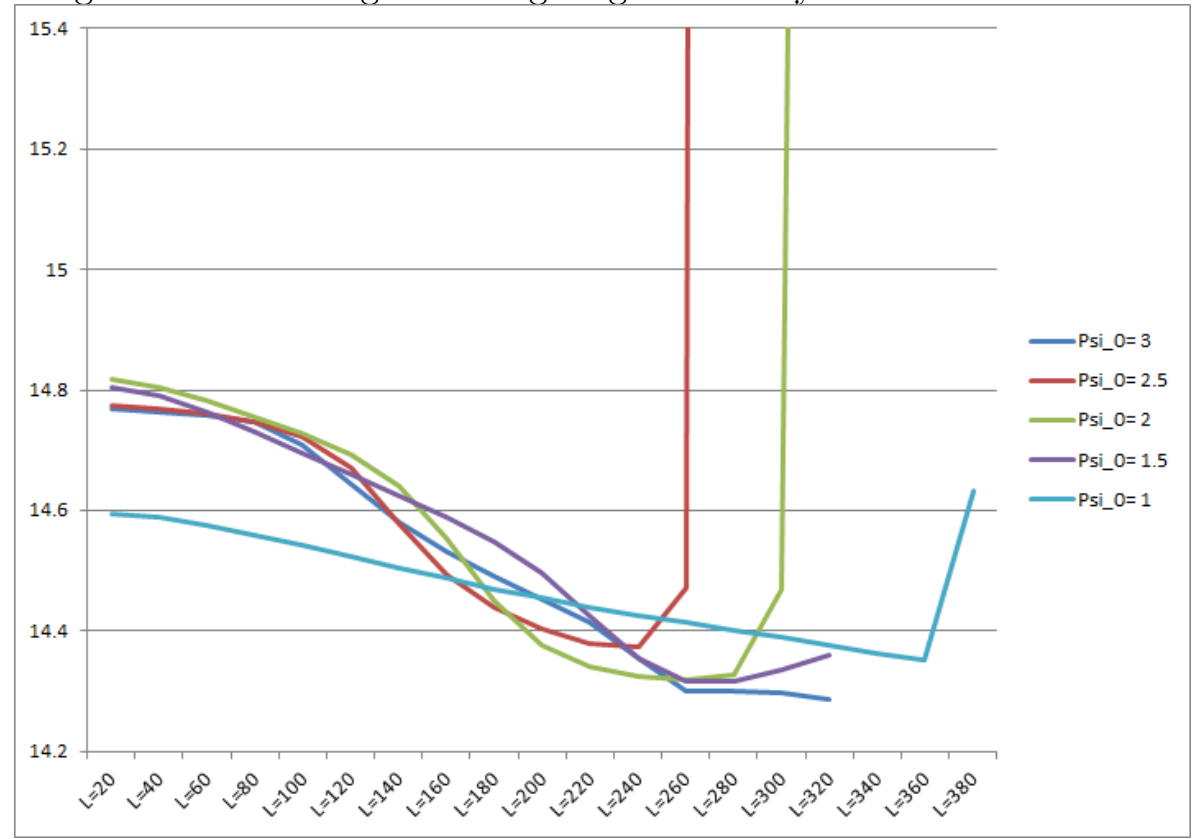

Figure 8.10: Root mean squared of the leveling height anomalies minus the quasigeoid computed with parameters $\psi_{0}$ and $L$. Scale is in $\mathrm{cm}$ 

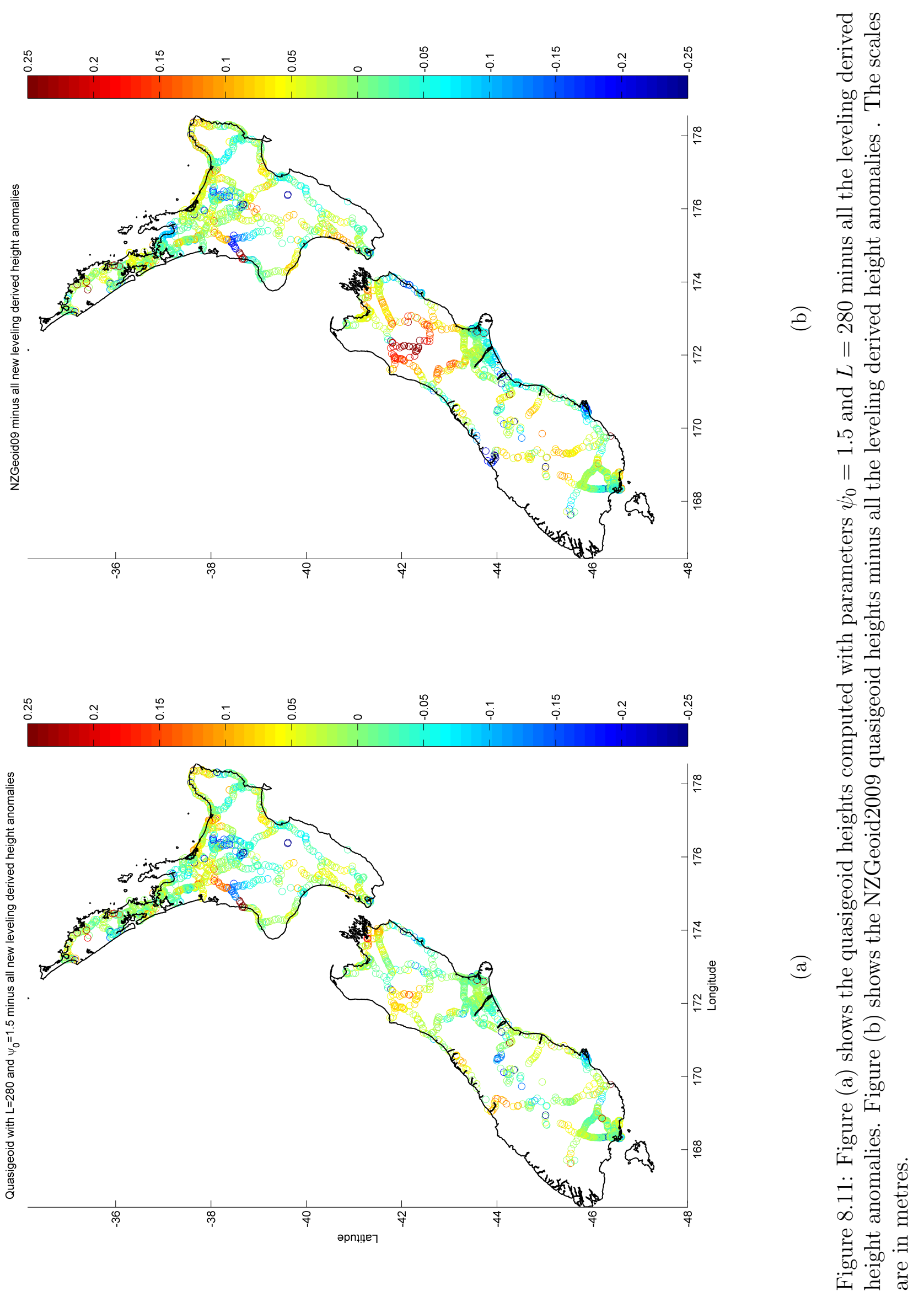
It can be seen here that the optimal parameter combination is again $\psi_{0}=1.5$ and $L=280$ determined by similar logic as before. At this minimum the standard deviation estimated from the inner $68 \%$ of the differences is $3.9 \mathrm{~cm}$ whereas using the smaller data set it is $3.8 \mathrm{~cm}$; this is a negligible change. The root mean squared residual here is 14.361 which is much larger than the $4.8 \mathrm{~cm}$ for the smaller leveling/GPS data set.

The standard deviations estimated from the inner $68 \%$ of both the larger and the smaller data sets are effectively the same, indicating a consistent accuracy around $3.8 \mathrm{~cm}$. The large root mean squared of around $14 \mathrm{~cm}$ is being caused by outliers in the additional leveling data. These points can be seen in figures $8.11 \mathrm{a}$ and $8.11 \mathrm{~b}$ of the new larger set of leveling derived height anomalies minus the quasigeoid computed with parameters $\psi_{0}=1.5$ and $L=280$ (after subtracting mean local vertical datum offsets), and the same using NZGeoid2009, respectively.

\subsubsection{Summary of results}

In this subsection the gridded residual gravity anomaly has been used to compute 80 different residual geoid undulations for parameters $L=20,40, \ldots, 320$ and $\psi_{0}=1^{\circ}, 1.5^{\circ}, 2^{\circ}, 2.5^{\circ}, 3^{\circ}$. The Eigen-6C4 geoid undulations and topographic effect have been added back to the residual geoids to obtain 80 quasigeoids.

Each of the 80 quasigeoids has been compared to the same leveling data used to evaluate the accuracy of NZGeoid2009. The best fitting quasigeoid had parameters of $L=280$ and $\psi_{0}=1.5$ and a standard deviation (estimated from the inner $68 \%$ of the differences) of $3.8 \mathrm{~cm}$ and root mean squared residual of $4.8 \mathrm{~cm}$. This is around a $1.25 \mathrm{~cm}$ improvement on NZGeoid2009. These statistics were calculated after subtracting a mean local vertical datum offset which are given in table 8.6.

The leveling derived height anomalies and gravimetric quasigeoid height differences were also inspected in 3 main urban areas, Auckland, Wellington and Christchurch. On average in these areas the differences had a standard deviation of $3.1 \mathrm{~cm}$ which is around $1.5 \mathrm{~cm}$ smaller than the $4.68 \mathrm{~cm}$ mean standard deviation derived for NZGeoid2009.

Finally the data have been compared to a new larger set of coupled leveling and GPS data. Over all the inner $68 \%$ of the differences between the large data set and the optimally chosen quasigeoid had a similar standard deviation to that of the smaller leveling database at $3.8 \mathrm{~cm}$ and the standard deviation for NZGeoid2009 was $5.44 \mathrm{~cm}$. However the root mean squared residual of the differences for the optimal new quasigeoid was around $14 \mathrm{~cm}$ and similarly 14.77 $\mathrm{cm}$ for NZGeoid2009. This is due to several anomalous points in the leveling data base which need to investigated and fixed or removed before these data can be used effectively. 


\subsection{Summary}

In this chapter the computational steps and results of a new gravimetric quasigeoid have been given.

The gridded gravity anomaly given at the end of section 7.5 has been transformed into a gridded Faye anomaly by restoring the effect of the Bouguer slab. Heights for this reverse correction were taken from a 1 arc minute grid of heights which were determined from a block averaged 8 $m$ digital elevation model.

The remove-compute-restore technique has been employed to determine the new gravimetric quasigeoid. Eigen-6C4 was demonstrated to provide the best fit to the leveling derived height anomalies over all other global gravity models in section 5.1 For this reason it was chosen as the reference model for the remove-compute-restore stages of the computation.

The Eigen-6C4 gravity anomaly was first subtracted from the gridded Faye anomaly data (remove) to obtain a residual gravity anomaly. Stokes integral was then evaluated using the residual gravity anomaly grid, with a Featherstone et al (1998) modified stokes kernel. The kernel modification degree $L$ and spherical integration cap $\psi_{0}$ were then varied over the ranges $L=20,40, \ldots, 320$ and $\psi_{0}=1^{\circ}, 1.5^{\circ}, 2^{\circ}, 2.5^{\circ}, 3^{\circ}$ to obtain 80 different residual quasigeoid height anomalies (compute). Finally the long wavelength quasigeoid height anomalies of Eigen-6C4 were then added back to the residual quasigeoid height anomalies and the primary indirect topographic effect was added (restore) to obtain 80 different regional quasigeoids.

Each quasigeoid was compared to the leveling derived height anomalies and overall the parameter combination $L=280$ and $\psi_{0}=1.5$ gave the best fitting quasigeoid, with a standard deviation (estimated from the inner $68 \%$ of the differences) of $3.8 \mathrm{~cm}$ and root mean squared residual of $4.8 \mathrm{~cm}$. This is around $1 \mathrm{~cm}$ better than the current official regional quasigeoid NZGeoid2009 and $0.77 \mathrm{~cm}$ better than the remove-compute-restore reference signal Eigen-6C4 in standard deviation.

The $L=280$ and $\psi_{0}=1.5$ quasigeoid was also evaluate in 3 main urban areas, Auckland, Wellington and Christchurch and had a standard deviation (again estimated from the inner $68 \%$ of the differences) of $3.976,3.385$ and $2.071 \mathrm{~cm}$ and root mean squared of $3.58,4.388$ and $4.572 \mathrm{~cm}$ respectively. These have an average of $3.1 \mathrm{~cm}$ std when considering the number of data points used in each region and it is a $1.5 \mathrm{~cm}$ improvement on the $4.68 \mathrm{~cm}$ average for NZGeoid2009.

Finally, the $L=280$ and $\psi_{0}=1.5$ quasigeoid was compared to a new larger set of leveling derived height anomalies which has only recently become available. Overall the standard deviation (again estimated from the inner $68 \%$ of the differences) was no different from that of the smaller data set, however the root mean squared was around $14 \mathrm{~cm}$. This result was consistent for NZGeoid2009 and indicates the new leveling data contain some extreme outliers which need to be removed before the data can be used to evaluate the quasigeoid effectively. 
N.B. Unlike the computation of NZGeoid2005 and NZGeoid2009 discussed in chapter 3 , the observed heights for the terrestrial gravity anomalies have not been adjusted for the estimated LVD offsets and the quasigeoid calculation repeated. This is because for NZGeoid2009 the iteration converged in two steps indicating that it is unnecessary. 


\section{Chapter 9}

\section{Summary, Research Outcomes and Recommendations for Future Work}

\subsection{Summary}

Theoretical Setting: Heights have been described mathematically in relation to approximations of the gravitational potential field of the Earth and Earth approximating ellipsoids. Normal-orthometric heights have been defined as the distance along the line orientated outward normal to an equipotential surface known as the quasigeoid and it was shown how the quasigeoid can be determined from physical measurements of the Earth's gravity gradient.

Knowing the quasigeoid separation from an Earth approximating ellipsoid, the normal-orthometric height can be calculated by subtracting it from an ellipsoidal height from a GPS device. These heights are easy to obtain and are important since they approximately describe how water will flow.

Background, vertical datums used in New Zealand: Until 2009, New Zealand had 13 official local vertical datums. There are discontinuities between the datums since they are each referenced to separate mean sea level estimations (from tide gauge measurements) which can differ by a constant. Each local vertical datum contains numerous leveling based height measurements and, as of 2009, 1422 of the leveling sites had had an ellipsoid height measured with a GPS device and so quasigeoid height anomalies can be determined. Grouping the data by one local vertical datum at a time, a mean offset from the quasigeoid can be evaluated. This process unifies the otherwise separate datums and was first put to practice by Amos (2007).

In 2009 an official regional quasigeoid for New Zealand, NZGeoid2009, was computed by Claessens et al (2009) with the intention of unifying the local vertical datums. By specifying the use of a normal-orthometric height system, NZGeoid2009 was further used as a reference surface to establish a new official single national vertical datum, NZVD2009. With this vertical datum, normal-orthometric heights can be determined using the NZGeoid2009 height and a GPS ellipsoidal elevation at any point within the region it covers. This is advantageous over leveling height transfers since it is less time consuming, however the accuracy of the determined heights can only ever be as good as the accuracy of NZGeoid2009.

The accuracy of NZGeoid2009 was evaluated by comparing it to the ellipsoidal and leveling height derived quasigeoid height anomalies after the mean local vertical datum offsets had been 
applied. The difference between the two data sets had a root mean squared residual of 6.144 $\mathrm{cm}$ and standard deviation (estimated from the inner $68 \%$ of the differences) of $4.8 \mathrm{~cm}$.

NZGeoid2009 was computed by evaluating Stokes' integral using the remove compute restore technique on gridded gravity data from the GNS Science terrestrial gravity database and a marine satellite altimetry gravity anomaly. It used EGM2008 to provide long wavelengths in the gravity field for the remove and restore stages of the computation.

The terrestrial gravity data NZGeoid2009 is based on have been collected over a time period of around 60 years. The measurements are generally accurate to $0.1 \mathrm{mGal}$, however the recorded heights of the points are quite uncertain due to the instrumentation used to obtain them (predominantly using barometers). The inaccuracies in the recorded heights degrade the quality of the determined gravity anomalies. Moreover the data were not collected with the intention of quasigeoid modelling, for this reason they are non uniform and particularly sparse in rough topography. Similarly the gravity data in all offshore areas came from the satellite altimetry marine gravity anomaly. Satellite altimetry is unreliable in shallow coastal areas since it is not possible to accurately determine sea level, this will degrade the quality of the derived quasigeoid in these areas.

The better the quality and density of the gravity measurements the more accurately the quasigeoid can be determined. To compute a more accurate quasigeoid for New Zealand the underlying data must be improved.

\section{Steps taken to improve the underlying gravity data for a new quasigeoid com-} putation: Initially, steps have been taken to improve the terrestrial gravity measurements. To ensure consistency in the historic terrestrial gravity data all of the calculations necessary to compute Bouguer anomalies have been performed again. This reduced the potential for any erroneous conversion formulas between ellipsoidal gravity corrections, which have given used in the database, to cause inconsistencies. More over terrain corrections have been recomputed utilizing a new $8 \mathrm{~m}$ resolution digital elevation model. These data have been appropriately assimilated with existing terrain corrections to obtain the best possible refined Bouguer anomaly observations from the data.

To improve upon the limitations of the previously available data coverage, an airborne gravity data set has been collected which covers New Zealand's North, South and Stewart Islands with a $10 \mathrm{~km}$ flight line spacing. These data are seamless between on and offshore areas and cover the rough topography uniformly. Unfortunately the data were susceptible to short erroneous sections of track due to poor flight conditions and the gravity profiles appeared to contain mean offsets from existing gravity models and between neighbouring lines. The bad data have been removed by carefully assessing the cross track agreement with other flight lines and with the global gravity model EGM2008, and offsets in the flight lines have been estimated by a least squares method which takes into consideration the spatially correlated gravity signal, following Reilly (1979) and Forsberg (1987).

The repeatability of the airborne data was assessed by collecting measurements along two separate calibration lines with 5 flights for each. The differences from a mean gravity anomaly along the lines had a standard deviation of approximately $2.5 \mathrm{mGal}$. The internal consistency of the data was assessed by evaluating the difference between flight line data at intersection points. This accuracy measure was shown to be susceptible to the along track filter, anisotropic 
topography and the relative flight line elevations. After correcting for all these effects the set of all intersecting differences had a standard deviation of approximately 5.9 mGal.

The airborne gravity data, recomputed terrestrial gravity data, shipborne gravity data (previously unused for NZGeoid2009) and new satellite altimetry gravity anomaly data were augmented into a single grid using least squares collocation and a logarithmic covariance function. This griding process optimally combined the separate data sets since it was able to appropriately deal with the different qualities of the input observations and handling the downward continuation of observations at different elevations (i.e. the airborne data). This grid has unprecedented accuracy and internal consistency and was compared to the gridded gravity data used to compute NZGeoid2009 explicitly.

Topographic effects were restored to the gridded data using a reverse Bouguer slab correction and a 1 arc-minute grid of elevations to obtain a gridded Faye anomaly. On comparison to the grid used to compute NZGeoid2009 the contribution of the airborne data is clear around shallow coastal areas and through rough topography through the centre of the South Island.

Investigating the accuracy of the most recent global gravity models to be used as the reference for a newly derived regional quasigeoid: The most recent global gravity models (since 2008 onwards) available from http://icgem.gfz-potsdam.de/ICGEM/ modelstab.html (last accessed September 2015) have each been compared to the terrestrial gravity data and the 1422 leveling and GPS derived quasigeoid height anomalies. Over all, after subtracting mean offsets for the local vertical datums, the Eigen-6C4 model fit the leveling and GPS derived quasigeoid height anomalies best whilst the EGM2008 model fit the terrestrial gravity data best.

The terrestrial gravity data is not an independent data set from the global gravity models and EGM2008 used the terrestrial data to determine a wider spectral range of spherical harmonic coefficients than Eigen-6C4 did, this is the reason it fits the gravity data better. Since the Eigen-6C4 gave the best fit to the leveling and GPS quasigeoid height anomalies, which is an independent data set, it was determined to be the most accurate model.

The computation of a new gravimetric quasigeoid: A new gravimetric quasigeoid has been computed from the gridded Faye anomaly by Stokes integral equation. The Featherstone et al (1998) modified Stokes kernel and the remove-compute-restore technique were used, with Eigen-6C4 as the reference gravity signal.

The Eigen-6C4 gravity model was subtracted from the gridded Faye anomaly (remove) and Stokes integral was evaluated on the residual gravity anomaly grid. A Featherstone et al (1998) modified Stokes kernel has been used and the modification degree $L$ and spherical cap for the integration $\psi_{0}$ were varied over the ranges $L=20,40,60, \ldots, 320$ and $\psi_{0}=1^{\circ}, 1.5^{\circ}, 2^{\circ}, 2.5^{\circ}, 3^{\circ}$. This resulted in 80 residual quasigeoid undulation grids. The computation took approximately 30 hours for each parameter variation and four instances of the program were run simultaneously. The Eigen-6C4 quasigeoid height anomalies were then added back to the residual quasigeoid undulation grids and the primary indirect topographic effect was restored to obtain 80 quasigeoid for each $L$ and $\psi_{0}$ parameter variation. Each quasigeoid was then compared to the leveling and GPS derived quasigeoid height anomalies after computing and subtracting LVD mean offset values. The optimal parameter choice was determined to be $L=280$ and $\psi_{0}=1.5$ which had the best agreement with the leveling and GPS derived quasigeoid height anomalies 
with a standard deviation of $3.8 \mathrm{~cm}$ and root mean squared of $4.8 \mathrm{~cm}$.

The $L=280$ and $\psi_{0}=1.5$ quasigeoid and leveling and GPS derived quasigeoid height anomalies agreement was also assessed closely in 3 main urban areas, Auckland, Wellington and Christchurch. Here there were 123, 169 and 125 data points respectively and the standard deviations were $3.976,3.385$ and $2.071 \mathrm{~cm}$ and root mean squared of $3.58,4.388$ and $4.572 \mathrm{~cm}$ respectively. This gives an average accuracy of $3.1 \mathrm{~cm}$ standard deviation in urban areas which is $1.5 \mathrm{~cm}$ better than NZGeoid2009.

\subsection{Research Outcomes}

Here the key research outcomes of this research project have been listed.

- The collection of a national airborne gravity dataset. These data provide gravity measurements with uniform coverage across the whole of New Zealand, including shallow coastal areas and parts of the country which have previously been impractical to survey. The main advantage of the airborne data over previous measurements is that it has been made using consistent instrumentations and so, on the whole, has a consistent accuracy.

- The accurate reduction of the terrestrial gravity data to refined Bouguer gravity anomalies. This process involved; computing the free air, normal gravity and Bouguer slab corrections using consistent formulas, estimating more accurate terrain corrections than what is provided in the data base by using an $8 \mathrm{~m}$ digital elevation model (which is significantly higher resolution than used for any other terrain corrections computed in the past), and computing an accuracy estimate for each gravity observation in the database by considering the discrepancies in the recorded heights.

- The most accurate global gravity model, which is currently available, in relation to the the leveling and GPS derived height anomalies in New Zealand has been determined to be Eigen-6C4. This is the first time some of the most contemporary global gravity models have been compared over New Zealand.

- The new airborne gravity data have been augmented with the refined terrestrial gravity measurements, the existing shipborne gravity data and a new high resolution satellite altimetry gravity anomaly by utilizing the method of least squares collocation. This resulted in a 1-arc minute grid of gravity anomaly data with unprecedented accuracy and internal consistency for quasigeoid modelling on comparison to that used for the computation of NZGeoid2005 (Amos, 2007) and NZGeoid2009 (Claessens et al, 2009).

- A set of 80 quasigeoids has been determined from the gridded gravity anomaly data. They were computed by Stokes integral equation using the Featherstone et al (1998) modified Stokes kernel and the remove-compute-restore technique, with Eigen-6C4 as the reference gravity signal. Each used a different parameter variation of the modification degree $L$ and $\psi_{0}$. Using the 1442 leveling and GPS derived quasigeoid height anomalies new local vertical datum offsets have been computed following the methodology of Amos (2007) for each quasigeoid. The local vertical datum offset corrected leveling and GPS derived quasigeoid height anomalies were then compared to the quasigeoids to determine which one they fit best to. The parameters combination $L=280$ and $\psi_{0}=1.5$ gave the best fit.

- A new quasigeoid ( $L=280$ and $\left.\psi_{0}=1.5\right)$ has been produced which has a significant improvement in the agreement with the leveling data over NZGeoid2009 and Eigen-6C4. 
In particular the new quasigeoid has an average accuracy of $3.08 \mathrm{~cm}$ in urban areas which is $1.6 \mathrm{~cm}$ better than for NZGeoid2009.

In summary, the airborne data acquisitions and careful treatment and augmentation of existing datasets has resulted in a gridded gravity anomaly which has been used to compute a new highly accurate quasigeoid. The new quasigeoid has been shown to be in better agreement with leveling and GPS derived quasigeoid height anomalies than the current official national quasigeoid NZGeoid2009, and has an estimated accuracy in urban areas of $3.08 \mathrm{~cm}$.

\subsection{Future Work}

Here, recommendations for future work are given.

\section{Computational Steps}

- To be in keeping with the computational procedure for NZGeoid2009, the Featherstone et al (1998) modified stokes kernel was used. This is because it is theoretically optimal. However it would be interesting to determine if it is optimal in practice by evaluating Stokes integral for other kernel modifications discussed in chapter 2.

- There was an implied bifurcation point in the agreement between the quasigeoid height anomalies and the leveling and GPS derived ones between Stokes integral spherical caps of $\psi_{0}=2.5$ and $\psi_{0}=3$. Here the root mean squared and standard deviation of the differences, with respect to the modification degree $L$, changed from being a smoothly decaying function with a distinct minimum, to a function which does not smoothly decay and has no minimum which is consistent for the root mean squared and the standard deviation. For this reason it would be advantageous to explore a finer range of parameters to better determine the optimal parameter combination of $L$ and $\psi_{0}$.

- The gravity anomaly data were gridded at 1-arc minute, which results in a quasigeoid gridded at the same resolution. When evaluating the fit of the quasigeoid to the leveling and GPS quasigeoid height anomalies, these gridded data must be interpolated to the leveling and GPS observation locations, and so there is potential here for aliasing of the quasigeoid height anomalies. It would be interesting to evaluate the effect of griding the gravity data at a finer resolution to investigate this effect, however this would have an impact on the computation time.

\section{Underlying data}

- As new global gravity models become available it would be advantageous to evaluate their accuracy with respect to the leveling and GPS derived quasigeoid height anomalies. If any are better than Eigen-6C4, they could be used as the reference signal for the remove-compute-restore technique to evaluate a new regional quasigeoid with better accuracy. Improvements in these models are mostly due to better satellite gravity data which contribute to the long wavelengths.

- It can be seen in the propagated errors that there is an area in Fiordland (in the South) where the error increases. This is due to the turbulence during the airborne data acquisition in this region and the sparse terrestrial data coverage. It would be advantageous to include more data in this region as they become available. This is also true of other areas where erroneous sections in the airborne data have been identified. 
- Substantial improvements have been given by the new quasigeoid near the coast and through the rough topography of the South Island, however most important is the improvement in urban areas. This is largely due to the inclusion of the airborne gravity data. If more highly accurate gravity observations could be acquired in these areas the quasigeoid could be determined even more accurately.

\section{Accuracy assessment}

- The quasigeoid accuracy was determined by a comparison to leveling and GPS derived height anomalies. The data set used to evaluated NZGeoid2009 had 1422 data points in 13 separate datums. Since 2009 approximately another 1400 points have been added to the database. However the new data set contains some extreme outliers which degrade the effectiveness of the quasigeoid and leveling data comparison. The erroneous data points need to be carefully identified and fixed, or removed, before the new data set can be used effectively.

- As more GPS ellipsoidal height observations are made at existing or new leveling height observation locations, they should be used to re-evaluate the accuracy of the quasigeoid.

In summary, as new gravity data become available they should be used to evaluate a new gridded gravity anomaly, perhaps at a finer resolution if appropriate for the spatial density of the new data, and newly released global gravity models should be constantly evaluated. The new gravity data and more accurate global gravity models should be used to calculate ever more accurate regional quasigeoids. It would advantageous to explore a wider range of Stokes kernel modification types, and a finer range of parameters variations. The accuracy assessment of the quasigeoid should also be ongoing so that as more reliable leveling and GPS derived quasigeoid height anomalies become available, regional quasigeoids can be assessed more accurately. 


\section{References}

Alberts, B A. Regional gravity field modelling using airborne gravity data.2009. Diss. TU Delft, Delft University of Technology.

Andersen, O. B. The DTU10 Gravity field and Mean sea surface 2010. Second international symposium of the gravity field of the Earth (IGFS2), Fairbanks, Alaska

Amos, M.J. Quasigeoid Modelling in New Zealand to Unify Multiple Local Vertical Datums 2007. PhD thesis, Curtin University of Technology, Perth, Australia.

Amos, M. J. and Featherstone, W. E.Comparisons of recent global geopotential models with terrestrial gravity field observations over New Zealand and Australia.. Geomatics Research Australasia. 79: 1-20. (2003)

Amos, M. J., W. E. Featherstone, and J. Brett 2005. Crossover adjustment of New Zealand marine gravity data, and comparisons with satellite altimetry and global geopotential models. 2005. In Gravity, Geoid and Space Missions, Volume 129, pp. 266271. IAG Symposia Series, Springer, Berlin, Germany.

Amos, M.J and Featherstone W.E., Preparations for a new gravimetric geoid model of New Zealand, and some preliminary results 2003, New Zealand Surveyor, No. 293, pp 3-14

Bell, Robin E., Coakley, Bernard J., Sternpt, Robert W. Airborne gravimetry from a small twin engine aircraft over the Long Island Sound, 1991. GEOPHYSICS, VOL. 56, NO.9 (SEPTEMBER 1991); P. 1486-1493.

Bae, T.S. Lee J. Kwon J.H. Hong, C. Update of the precision geoid determination in Korea, 2012, Geophysical Prospecting Volume 60, Issue 3, pages 555571.

Brozena, J. The Greenland Aerogeophyscis Experiment, Airborne Gravity, Topographic and Magnetic Mapping of an entire Continent 1991. From Mars to Greenland: Charting Gravity with Space and Airborne Instruments, edited by O. Colombo, IAG Symposium Series 110, pp. 203214, Springer Verlag.

Bruinsma, S., Foerste, C., Abrikosov, O., Marty, J.-C., Rio, M.-H., Mulet, S., Bonvalot, S. The new ESA satellite-only gravity field model via the direct approach, 2013, Geophysical Research Letters, 40, 14, p. 3607-3612.

Bruns, H. Die Figur der Erde, 1878. Publ. Konigl. Preuss, Geod. Insittute, P. Stankiewicz Buchruckerei, Berlin, Germany.

Bruton AM Improving the accuracy and resolution of SINS/DGNSS airborne gravimetry 2000. Ucge report 20145, Department of Geomatics Engineering, University of Calgary, Calgary, Canada. 
Chein-Way Hwang, Hsin-Ying Hsu. SHALLOW-WATER GRAVITY ANOMALIES FROM SATELLITE ALTIMETRY: CASE STUDIES IN THE EAST CHINA SEA AND TAIWAN STRAIT 2008. Journal of the Chinese Institute of Engineers, Vol. 31, No. 5, pp. 841-851

Claessens, S.J. Hirt, C. Featherstone, W. Kirby. J Computation of a new gravimetric quasigeoid model for New Zealand 2009. Western Australian Centre for Geodesy Curtin University of Technology.

Claessens, S.J. Featherstone, W.E. Anjasmara, I.M. Filmer, M.S. Is Australian data really validating EGM2008, or is EGM2008 just in/validating Australia data? 2009b. Newton's Bulletin. 4: pp. 207-251.

Childers V., Bell R., Brozena J.Airborne gravimetry: an investigation of filtering 1999, Geophysics, 64, 6169.

Dehlinger, P. Marine gravity 1978. Elsevier.

Ekman, M. Impacts of geodynamic phenomena on systems for heights and gravity. 1989. Bulletin Godsique, Vol. 63, No. 3.

Duquenne, H., M. Everaerts, and P. Lambot Merging a gravimetric model of the geoid with GNSS/leveling data: an example in Belgium 2005. In Gravity, Geoid and Space Missions, Volume 129, pp. 131136. IAG Symposia Series, Springer, Berlin, Germany.

Featherstone, W.E., Evans, J.D. and Olliver, J.G. A Meissl-modified Vanicek and Kleusberg kernel to reduce the truncation error in gravimetric geoid computations. 1998. Journal of Geodesy, Vol. 72, No. 3.

Featherstone, W.E., Kirby, J.F., Kearsley, A.H.W., Gilliland, J.R., Johnston, G.M., Steed, J., Forsberg, R. and Sideris M.G. The AUSGeoid98 geoid model of Australia: data treatment, computations and comparisons with GPS-leveling data. 2001. Journal of Geodesy, Vol. 75, No. $5-6$.

Featherstone, W.E. and Kirby, J.F. The reduction of aliasing in gravity anomalies and geoid heights using digital terrain data. 2000. Geophysical Journal International, 141(1): 204-212, doi:10.1046/j.1365-246X.2000.00082.x.

Featherstone, W.E. and Kuhn, M. Height systems and vertical datums: a review in the Australian context. 2006. Journal of Spatial Science, Vol. 51, No. 1, pp.21-41.

Featherstone, W. E., and M. C. Dentith, A geodetic approach to grav-ity data reduction for geophysics 1997 Computational Geosciences, 23, 10631070, doi: 10.1016/S0098-3004(97)000927 .

Fenoglio-Marc L., Vignudelli S., Humbert A., Cipollini P., Fehlau M. , Becker M. $A N A S$ SESSMENT OF SATELLITE ALTIMETRY IN PROXIMITY OF THE MEDITERRANEAN COASTLINE. 2008. In 3rd ENVISAT symposium proceedings, SP-636, ESA Publications Division.

Forsberg, R. A New Covariance Model for Inertial Gravimetry and Gradiometry. 1987. JOURNAL OF GEOPHYSICAL RESEARCH, VOL. 92, NO. B2, PAGES 1305-1310.

Forsberg R. Kenyon, S. Downward continuation of airborne gravity data. 1995. Proc. Of Airborne Gravimetry, 2-14. 
Forsberg R, Olesen A, Munkhtsetseg D, and Amarzaya A Downward continuation and geoid determination in Mongolia form airborne and surface gravimetry and SRTM topography. 2007. Proceedings of the 1st International Symposium of the International Gravity Field Service Gravity field of the Earth.

Forsberg, R., Olesen, A. V., Keller, K., Mller, M., Gidskehaug, A., and Solheim, D. Airborne gravity and geoid surveys in the Arctic and Baltic Seas 2001. In Proceedings of international symposium on kinematic systems in geodesy, geomatics and navigation (KIS-2001), Banff (pp. 586-593).

Forsberg R, Olesen A, Keller K, Airborne Graivty Survey of North Greenland Shelf 19981999. Technical Report no.10, Kort og Matrikelstyrelsen, Copenhagen.

Forsberg R, Olesen A. V, Einarsson I, Airborne gravimetry for geoid determination with Lacoste Romberg and Chekan gravimeters 2015, Gyroscopy and Navigation October 2015, Volume 6, Issue 4, pp 265-270

Forsberg R An overview manual for the GRAVSOFT Geodetic Gravity Field Modelling Programs 2003, DNSC Daniosh National Space Center, DRAFT 1.ed September 2003.

Forsberg, R., Olesen, A. V., Keller, K., Mller, M., Gidskehaug, A., and Solheim, D. Airborne gravity and geoid surveys in the Arctic and Baltic Seas. 2001b In Proceedings of international symposium on kinematic systems in geodesy, geomatics and navigation (KIS-2001), Banff (pp. 586-593).

Forste C, Bruinsma S, Abrykosov O, Lemoine J-M, The latest combined global gravity field model including GOCE data up to degree and order 2190 of GFZ Potsdam and GRGS Toulouse. 2015. GFZ data services http://dx.doi.org/10.5880/icgem.2015.1

Forste Ch, Bruinsma S.L, Abrikosov O, Lemoine J-M, Schaller T, Gtze H-J, Ebbing J, Marty J-C, Flechtner F, Balmino G and Biancale R, EIGEN-6C4 The latest combined global gravity field model including GOCE data up to degree and order 2190 of GFZ Potsdam and GRGS Toulouse, 2014, http://icgem.gfz-potsdam.de/ICGEM/documents/Foerste-et-alEIGEN-6C4.pdf (last accessed 2015).

GrafNav / GrafNet User Guide 2013, Publication Number: OM-20000147, Revision Level: 1.

Glennie, C. L., Schwarz, K. P., Bruton, A. M., Forsberg, R., Olesen, A. V., and Keller, K. (2000)A comparison of stable platform and strapdown airborne gravity 2000. Journal of Geodesy, 74(5), 383-389.

Gruber, T. Evaluation of the EGM2008 Gravity Field by Means of GPS/leveling and Sea Surface Topography Solutions. 2009. Newtons Bull 4:317. Bureau Gravimtrique International (BGI)/International Geoid Service (IGeS). ISSN:18108555

Hammer, S. Terrain corrections for gravimeter stations 1939. Geophysics, Vol. 4, No. 3, pp. 184-194.

Hannah, J. A long-term sea level change and its implications for geodetic networks 1989, Mar. Geod., 13(2), 91100, doi:10.1080/15210608909379613.

Hannah, J. Analysis of mean sea level data from New Zealand for the period 189919881990 , J. Geophys. Res., 95(B8), 12,39912,405, doi:10.1029/JB095iB08p12399. 
Hannah, J. An updated analysis of long-term sea level change in New Zealand 2004, Geophys. Res. Lett., 31, L03307, doi:10.1029/2003GL019166.

Hannah, J., P. H. Denys, and J. Beavan The determination of absolute sea level rise in New Zealand 2010, abstract G53A0708 presented at 2010 Fall Meeting, AGU, San Francisco, Calif., 1317 Dec.

Hannah, J. Bell, G. Regional sea level trends in New Zealand 2012, JOURNAL OF GEOPHYSICAL RESEARCH, VOL. 117, C01004, doi:10.1029/2011JC007591.

Harlan, R.B. Eotvos Corrections for Airborne Gravimetry 1968. J. Geophysics Res.. 14,17.

Heck, B. and Grninger, W. Modification of Stokess integral formula by combining two classical approaches 1987. Proceedings of the XIX General Assembly of the International Union of Geodesy and Geophysics, Vol. 2, Vancouver, Canada,

Helmert, F.R. Die Schwerkraft im Hochgebirge insbesondere in den Tyroler Alpen in geodtischer und geologischer Beziehung 1890. Verffentlichung Knigliche Preussiche Geodtischen Institutes und Centralbureaus der Internationalen Erdmessung., No. 1, Berlin, Germany, 52 pp.

Heiskanen, W.A. and Moritz, H. Physical Geodesy 1967. W.H. Freeman and Company, San Francisco, USA, 364 pp.

Hirt, C. Assessment of EGM2008 over Germany using accurate quasigeoid heights from vertical deflections, 1 GCG05 and GPS/leveling 2012. Zeitschrift fur Geodasie, Geoinformation und Landmanagement zfv) 136(3): 138-149.

Huang, J. Vronneau, M. Assessments of Recent GRACE and GOCE Release 5 Global Geopotential Models in Canada 2015. Newton's Bulletin, Issue n. 5, June 2015.

Hwang C, Hsiao Y.S, Shih H.C, Yang M, Chen K.H, Forsberg R, Olesen A.V. Geodetic and geophysical results from a Taiwan airborne gravity survey: Data reduction and accuracy assessment 2007. JOURNAL OF GEOPHYSICAL RESEARCH, VOL. 112.

Hwang, C.W Hsu, H.Y. Shallow-water gravity anomalies from satellite altimetry: Case studies in the east China and Taiwan Strait 2008. Journal of the Chinese Institute of Engineers, Vol. 31, No. 5, pp. 841-851.

Hwang, C., Hsiao, Y. S., Shih, H. C., Yang, M., Chen, K. H., Forsberg, R., Olesen, A. V. Geodetic and geophysical results from a Taiwan airborne gravity survey: Data reduction and accuracy assessment 2007. Journal of Geophysical Research: Solid Earth, 112(B4).

Hwang, C., Hsiao, Y. S., and Shih, H. C.Data reduction in scalar airborne gravimetry: Theory, software and case study in Taiwan 2006. Computers and geosciences, 32(10), 1573-1584.

Huang, Y., Olesen, A. V., Wu, M., and Zhang, K. SGA-WZ: A new strapdown airborne gravimeter 2012. Sensors, 12(7), 9336-9348.

Jeffreys, H An alternative to the rejection of observations 1932, Proc Soc. London, Series A,137, $77-87$

Jeffreys, H Approximate methods and simplifications 1961, Theory of probability. 3rd ed. Oxford: Claredon Press. 
Jekeli, C, Yang, H.J, Kwon, J.H Geoid Determination in South Korea from a combination of Terrestrial and Airborne Gravity Anomaly Data 2013. Journal of the Korean Society of Surveying, Geodesy, Photogrammetry and Cartography, Vol. 31, No. 6-2, pp. 567-576.

Kasper, J. F. A second-order Markov gravity anomaly model. 1971. Journal of Geophysical Research 76(32), 78447849.

Kearsley, A. H. W. Non-stationary estimation in gravity prediction problems. 1977. Report 256, Department of Geodetic Science, The Ohio State University, Columbus, USA.

Kirby, J.F. and Featherstone, W.E. High resolution grids of gravimetric terrain corrections and complete Bouguer gravity reductions over Australia 2002. Exploration Geophysics, Vol. 33, No. 3-4, pp. 161-165.

Klingele, E., Cocard, M., Halliday, M., and Kahle, H.G. The airborne gravimetric survey of Switzerland 1996. Swiss Geophys. Commission.

Kwon, J. H., and Jekeli, C. A new approach for airborne vector gravimetry using GPS/INS. 2001, Journal of Geodesy, 74(10), 690-700.

LaCoste, L. J. Measurement of gravity at sea and in the air 1967. Reviews of Geophysics, 5(4), 477-526.

Lowrie, W. Fundamentals of geophysics 2007. Cambridge University Press.

Mader, K. Die orthometrische Schwerekorrektion des Przisions- Nivellements in den Hohen Tauren (The orthometric weight correction of precision leveling in high terrain) 1954. sterreichische Zeitschrift fr Vermessungswesen, Sonderheft 15, Vienna.

Meissl, P. Preparations for the numerical evaluation of second-order Molodensky-type formulas 1971. Report 163, Department of Geodetic Science and Surveying, Ohio State University, Columbus, USA.

Mertikas, S. P.Gravity, Geoid and Earth Observation 2010. IAG Commission 2: Gravity Field, Chania, Crete, Greece, 23-27 June 2008 (Vol. 135). Springer Science and Business Media.

Model 'S' Air Sea Dynamic Gravity Meter System II, Instruction Manual March 2006, Micro-g LaCoste 1401 Horizon Avenue, Lafayette, Colorado 80026 USA.

Moritz, H. Advanced least squares method 1972. Report No. 75, Dept. of Geodetic Science,OSU

Moritz, H. Geodetic Reference System 1980 1980. Bulletin Godsique, Vol. 54, No. 3, pp. 395405, doi:10.1007/BF02521480.

Murray, A.S., and Ray M. T. "Best Practice in Gravity Surveying." 2001, Australian Geological Survey.

Nagy, D., The gravitational attraction of a right angular prism. 1966. Geophysics, Vol. 31, No. 2, pp. 362-371.

Neda, D. Modification of the least-squares collocation method for non-stationary gravity field modelling. 2009. Ph.D. Curtin University of Technology, Department of Spatial Sciences

Niethammer, T. leveling and weight as means for the computation of true sea level heights 1932. Schweizersche Geodtische Kommission, Berne, 76 pp. 
Nowell, D.A.G. Gravity terrain corrections an overview. 1999. Journal of Applied Geophysics, Vol. 42, No. 2, pp. 117-134.

Olesen A.V Improved airborne scalar gravimetry for regional gravity field mapping and geoid determination. 2002. PhD Thesis, University of Copenhagen.

Olesen, A. V., Forsberg, R., Keller, K., Kearsley, A. H. W. . Error sources in airborne gravimetry employing a spring-type gravimeter.2002, In Vistas for Geodesy in the New Millennium (pp. 205-210). Springer Berlin Heidelberg.

Pavlis, N.K., S.A. Holmes, S.C. Kenyon, and J.K. Factor An Earth Gravitational Model to Degree 2160: EGM2008 2008. 2008 General Assembly of the European Geosciences Union, Vienna, Austria.

Peters, M.F., Brozena, J.M.,Methods to improve existing shipboard gravimeters for airborne gravimetry, K.-P. Schwartz, J.M. Brozena, G.W. Hein, (Eds), IAG Symposium on Airborne Gravity Field Determination, IUGG XXI General Assembly, Calgary, Boulder, CO, pp.39-45. (1995)

Rapp, R.H. Use of potential coefficient models for geoid undulation determinations using a spherical harmonic representation of the height anomaly/geoid undulation difference 1997, Journal of Geodesy, Vol. 71, No. 5, pp. 282-289, doi: 10.1007/s001900050096.

Reilly, W.I. Mapping the local geometry of the Earth's gravity field 1979. Report No.143, Geophysics dvision, Department of Scientific and Industrial Research New Zeland.

Sjberg, L.E. On the quasigeoid to geoid separation 1995, manuscripta geodaetica, Vol. 20, No. 3, pp. 182-192.

Stagpoole, V. Description of the data in the GNS Science science gravity database May 2012 GNS Science science internal report.

Sandwell, D. T., R. D. Mller, W. H. F. Smith, E. Garcia, R. Francis. New global marine gravity model from CryoSat-2 and Jason-1 reveals buried tectonic structure 2014, Science, Vol. 346, no. 6205, pp. 65-67, doi: 10.1126/science.1258213, 2014.

Schwarz K P, et al. The use of FFT techniques in physical geodesy 1990. Geophys. J. International, 1990, 100: 485-514

Shaw, L., I. Paul, and P. Henrikson. Statistical models for the vertical deflection from gravityanomaly models1969. Geophysical Journal International 74(17), 42594265.

Shi P, Sun Z M. The Solution to the problem of the spherical interior Dirichlet and its application 1999. Acta Geodaetica et Cartographica Sinica, 1999, 28(3):

Shih, H. C., Hwang, C., Barriot, J. P., Mouyen, M., Corria, P., Lequeux, D., and Sichoix, L. High-resolution gravity and geoid models in Tahiti obtained from new airborne and land gravity observations: data fusion by spectral combination. 2015. Earth, Planets and Space, 67(1), 1.

Telford, W. M., Geldart, L. P., and Sheriff, R. E. Applied geophysics 1990. (Vol. 1). Cambridge university press.

Tikhonov A N, et al.Solution of Ill-Posed Problem 1977. New York: John Wiley and Arsenin 
Tth, G. New combined geoid solution HGTUB2007 for Hungary 2009. In Observing our Changing Earth (pp. 405-412). Springer Berlin Heidelberg.

Vanicek, P. and Kleusberg, A. The Canadian geoid Stokesian approach 1987. manuscripta geodaetica, Vol. 12, No. 2

Wang Xing-Tao,XIA,Zhe-Ren,SHI Pan and SUN Zhong-Miao. Methods for airborne gravity data 2004. CHINESE JOURNAL OF GEOPHYSICS Vol.47, No.6, 2004, pp: 11431149.

Wessel, P. and Watts, A.B. On the accuracy of marine gravity measurements, 1998. Journal of Geophysical Research, Vol. 94, No. B4, pp. 7685-7729.

Wichiencharoen, C. The indirect effects on the computation of geoid undulations 1982. Report 336, Department of Geodetic Science and Surveying, Ohio State University, Columbus, USA.

Wong, L. and Gore, R. Accuracy of geoid heights from modified Stokes kernels 1969. Geophysical Journal of the Royal Astronomical Society, Vol. 18, pp. 81-91.

Woodward, D. J. and Carman, A. F. Computer program to reduce precise gravity observations. 1984. Geophysics Division Technical Note No 93, DSIR Wellington

Yang, H.J. Geoid Determination Based on a Combination of Terrestrial and Airborne Gravity Data in South Korea. 2013, Report No. 507, Geodetic Science, The Ohio State University, Columbus, Ohio 43210.

Zwillinger, D, Kokoska, S CRC Standard Probability and Statistics Tables and Formulae 2010. CRC Press. p. 49. ISBN 978-1-58488-059-2. 


\section{Appendix A}

\section{Calculating the Lacoste and Romberg model 'S80' gravimeter accelerometer MV to mGal scale factor}

\section{A.1 Introduction}

The Lacoste and Romberg model 'S80' has two accelerometers (one pointing across the meter, $X A C C$, and one along the meter, $L A C C$ perpendicular to the horizontal) which are used in conjunction with the gyros and torque motors to level the platform and calculate the cross coupling correction.

The readings from the accelerometers are included in the gravimeter output file but are given in millivolts. The millivolt (MV) readings can be transformed into mGal using a scale factor which must be calculated. Here, the methodology and the results of an experiment to compute this scale factor are given.

\section{A.2 Methodology}

The methodological step to calculate the scale factor for a single accelerometer.

1. Record the absolute gravity at the location of the gravimeter, g.

2. Record the spring tension when the gravimeter is level (i.e. the accelerometer reading should be approximately zero), $S_{0}$.

3. Tilt the gravimeter through one axis holding the other fixed and record the new spring tension value, $S_{n}$ and accelerometer reading for the axis that is being titled. e.g. $X A C C_{n}$ for the cross accelerometer.

4. Repeat step 3 for various tilt angles, at each stage recording the spring tension and accelerometer readings.

5. Calculate $\Delta g_{n}=0.9899 *\left(S_{n}-S_{0}\right)$ for each measurement (where 0.9899 is the counter units to $\mathrm{mGal}$ scale factor). 
6. Calculate the tilt angle $\theta_{n}=\cos ^{-} 1\left(1-\frac{\Delta g_{n}}{g}\right)$ for each measurement.

7. Calculate $A_{n}=g \sin \left(\theta_{n}\right)$. This is an approximation of the accelerometer reading in $\mathrm{mGal}$ derived solely from the vertical gravity measurement.

8. The mean scale factor $k$ such that $k \times X A C C=A$ is then given by,

$$
\frac{\sum_{n=1}^{N}\left|X A C C_{n}\right| / A_{n}}{N}
$$

where $\mathrm{N}$ is the number of measurements made.

Figure A.1 gives a graphical representation of the process described above.

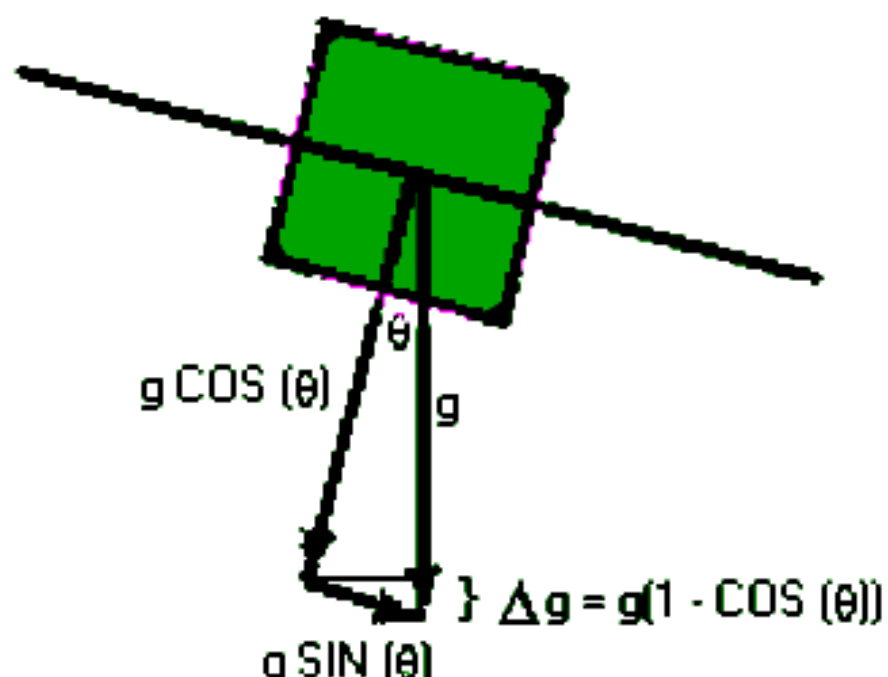

$$
\begin{aligned}
& \theta=\operatorname{COS}^{-1}(1-\Delta g / g) \\
& A=g \operatorname{SIN}(\theta)
\end{aligned}
$$

Figure A.1: Graphical representation of the change in the strength of gravity due to a tilt. Adapted from Model 'S' Air Sea Dynamic Gravity Meter System II, Instruction Manual (2006) 


\section{A.3 Results}

The gravimeter was tilted to various angles whilst retaining one of the axes as close to perpendicular to the vertical gravity gradient as possible. Tables A.1 and A.2 demonstrate the recorded measurements for each of the accelerometers for various tilt angles in MV, the recorded spring tension, derived tilt angle $\theta_{n}$ and calculated values $A_{n}$, in accordance with the computational steps described in the previous section.

Table A.1: Results of tilting the gravimeter through an axis parallel to the XACC accelerometer to record changes in the LACC accelerometer.

\begin{tabular}{|l|l|l|l|l|}
\hline XACC & LACC & Spring tension & $\theta$ & A \\
\hline 10.4 & 8.2 & 10611.6 & 0 & 0 \\
\hline 19.1 & 291.2 & 10588.1 & 0.00688 & 6753.27 \\
\hline 14.1 & 976.6 & 10289.5 & 0.02550 & 25000.17 \\
\hline 10.1 & 1802.3 & 9493.2 & 0.04753 & 46575.65 \\
\hline 11.1 & 659.8 & 10479.3 & 0.01634 & 16023.17 \\
\hline 17.3 & -304.8 & 10576.2 & 0.00845 & 8288.59 \\
\hline 19.7 & -622.2 & 10406.7 & 0.02034 & 19940.29 \\
\hline 7.2 & -1031.2 & 10205.5 & 0.02863 & 28070.81 \\
\hline
\end{tabular}

Table A.2: Results of tilting the gravimeter through an axis parallel to the LACC accelerometer to record changes in the XACC accelerometer.

\begin{tabular}{|l|l|l|l|l|}
\hline XACC & LACC & Spring tension & $\theta$ & A \\
\hline 15.6 & 13.9 & 10611.6 & 0 & 0 \\
\hline 110.0 & 13.5 & 10609.0 & 0.00229 & 2246.30 \\
\hline 268.5 & 13.7 & 10589.5 & 0.00668 & 6549.02 \\
\hline 533.3 & 13.8 & 10517.4 & 0.01379 & 13520.66 \\
\hline 909.3 & 13.8 & 10331.5 & 0.02378 & 23313.57 \\
\hline-108.3 & 13.8 & 10607.0 & 0.00304 & 2987.86 \\
\hline-257.7 & 13.6 & 10585.2 & 0.00730 & 7157.84 \\
\hline-548.6 & 13.7 & 10497.6 & 0.01517 & 14873.83 \\
\hline-843.3 & 13.7 & 10448.1 & 0.01817 & 17812.46 \\
\hline-1299.6 & 13.5 & 9996.2 & 0.03525 & 34553.67 \\
\hline
\end{tabular}

From the data recorded in these tables, a least squares solution to the conversion factor $k$ has been determined for each of the accelerometers. For the LACC accelerometer the estimated conversion factor is 26.4830 and for the XACC accelerometer the estimated conversion factor is 25.1101. Figures A.2 and A.3 show the absolute value of the accelerometer value and derived value $A$ for both the LACC and XACC accelerometer and the lines of best fit which have a gradient of $k$. 


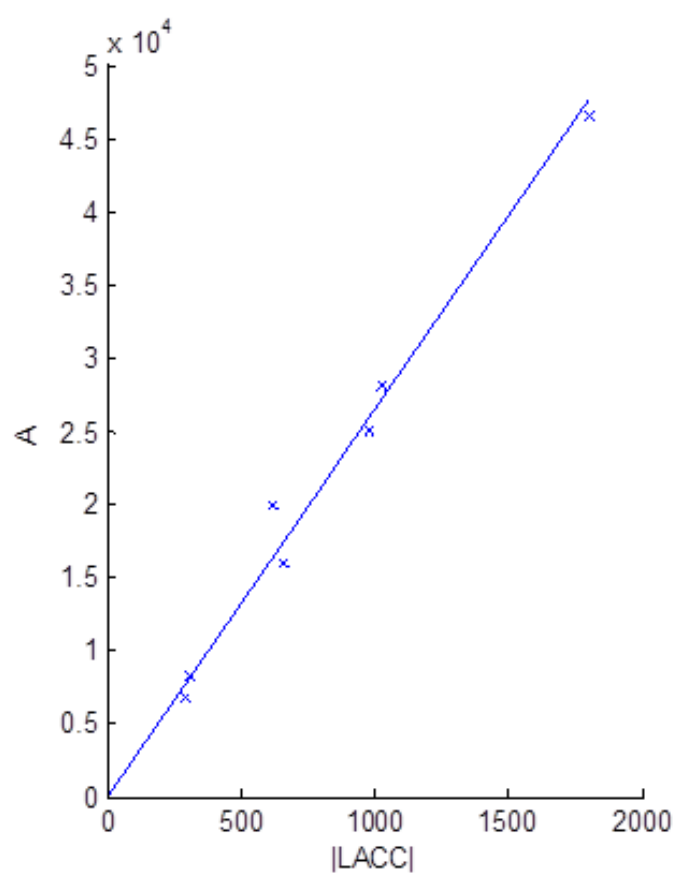

Figure A.2: The absolute value of the LACC accelerometer recording and derived value $A$ for various tilt angles a line of best fit with gradient $k=26.4830$.

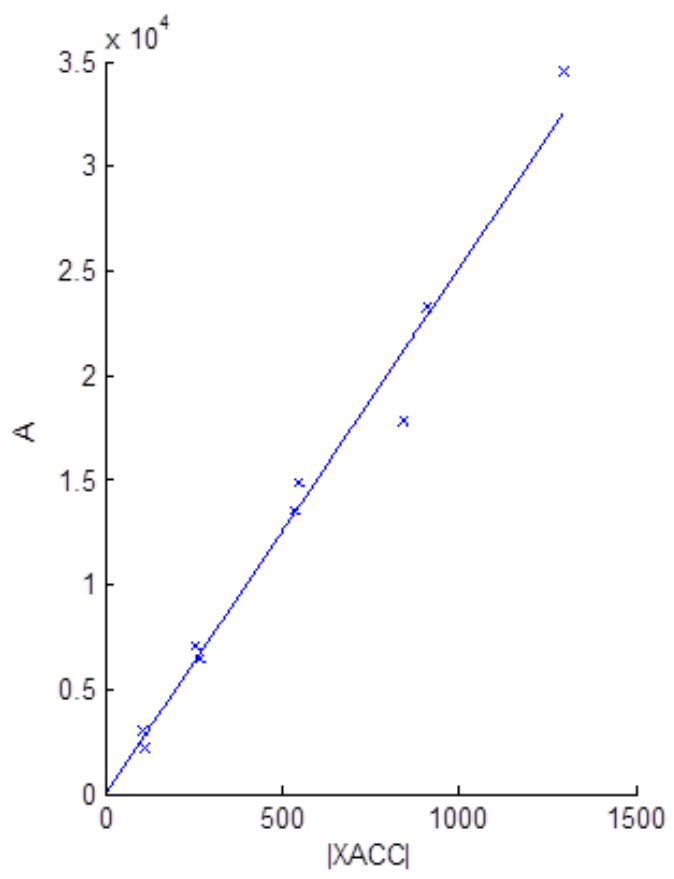

Figure A.3: The absolute value of the XACC accelerometer recording and derived value $A$ for various tilt angles a line of best fit with gradient $k=25.1101$. 


\section{Appendix B}

\section{Fitting Gaussian distributions to leptokurtotic data sets}

Throughout this research project it has been necessary to measure the "goodness of fit" of mathematical models to real life data. The differences between models and data, here denoted $x$, have been assumed to follow a Gaussian distribution which is parameterized by a mean, $\mu$ and a standard deviation, $\sigma$.

The assumption that the differences, $x$, are Gaussian is fair for a large central portion of the empirical distributions but in many instances there are large outliers on the tails. These outliers result in unrealistically large estimated standard deviations, $\sigma$, for the empirical distributions when the standard deviation is calculated by the standard formula, given by equation B.1. These large standard deviation estimates do not represent the majority of the residuals particularly well.

$$
\sigma=\sqrt{\frac{\sum_{i=1}^{i=N}\left(x_{i}-\mu\right)^{2}}{N}}
$$

A method here is presented which accurately estimates the standard deviation of an underlying dataset in the presence of outliers. Most importantly it is robust since it does not underestimate the standard deviation if the assumption that outliers are present is false.

\section{B.1 Standard methodology}

Consider $X$ to be a set of random values drawn from a Gaussian distribution with a zero mean and standard deviation of 5 (e.g. this could be the set of cross over discrepancies discussed in section 6 of the difference between the quasigeoid and the leveling/GPS data described in section 3 ). Suppose as well there is a set of $Y$ values which are also drawn from a Gaussian distribution with a zero mean, but this time a standard deviation of 20 (e.g. In the context of section 6 these could be larger cross over discrepancies where one of the flight lines has been affected by turbulence). In reality often datasets which are assumed to be Gaussian are actually a mix of data which consists mostly of values drawn from the $X$ distribution but in addition a handful of values also drawn from the $Y$ distribution (e.g. with the cross over discrepancies most were around $5 \mathrm{mGal}$, but on occasion several intersection points were not reliable due to extreme factors).

The standard deviation as estimated by equation B.1 will actually be mixture of the stan- 
dard deviation of $X$ and the standard deviation of $Y$ depending on how many values from each distribution you have. i.e if you have 90 data points from the $X$ distribution and 10 data points from the $Y$ distribution, an empirical standard deviation using equation B.1 will give a $\sigma$ value close to $\sqrt{5^{2} * 90 / 100+20^{2} * 10 / 100} \approx 7.9$. The standard deviation of the mixed distribution is much larger than data drawn from the distribution $X$ and so the true distribution of most of the data is heavily masked by the influence of a few bad points.

A standard approach to estimating standard deviations in the presence of outliers is by excluding all values from the distribution larger in magnitude than some fixed value, for example using a confidence interval - where only an inner percentage of the data is considered. This approach has an inherent danger since it will always result in an under estimation of the true standard deviation if the assumption that there are outliers is false. The under estimation worsens as the size of the confidence interval shrinks. The following figure depicts this, a set of 10,000 values has been chosen at random from a Gaussian distribution with a zero mean and standard deviation of 5 , data from an inner percentage (varied from 0 to $100 \%$ ) is excluded and the standard deviation of the selected portion of the data is computed by equation B.1.

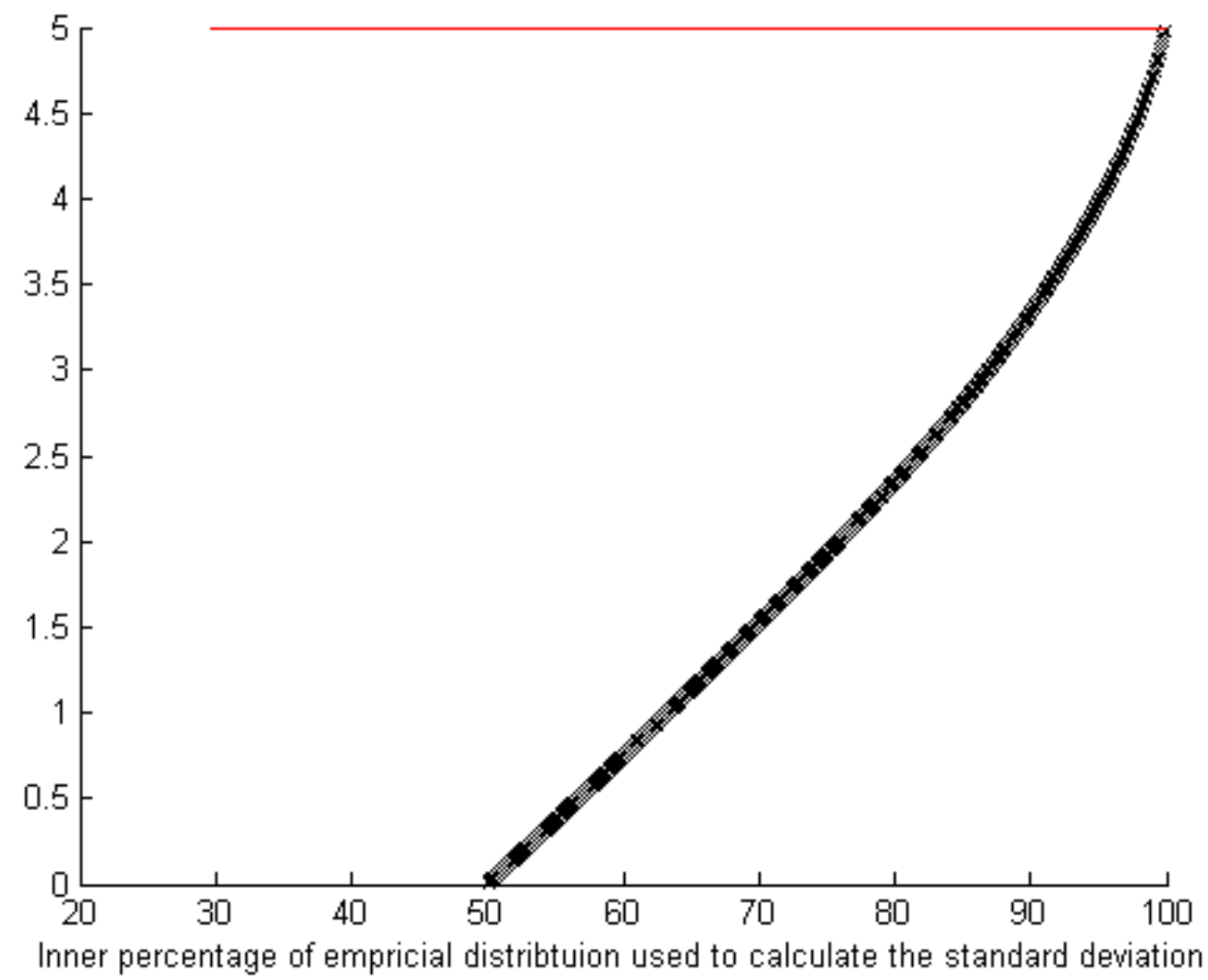

Figure B.1: Standard deviation (y-axis) calculated from inner percentage (x-axis) of Gaussian distribution with a standard deviation of 5 . 


\section{B.2 Derivation of an alternative method to derive the standard deviation of an empirical distribu- tion in the presence of outliers.}

Building on Jeffereys (1932 and 1961), a Gaussian density function, $F(\hat{x})=P(x<\hat{x})$, gives the probability of drawing some value, $x$, is less than $\hat{x}$, where (for $x$ with a zero mean),

$$
F(\hat{x})=\int_{-\infty}^{\hat{x}} \frac{1}{\sqrt{2 \pi} \sigma} e^{-x^{2} / 2 \sigma^{2}} d x
$$

and the variance, $\sigma^{2}$ is given by,

$$
\sigma^{2}=\int_{-\infty}^{\infty} x^{2} \frac{1}{\sqrt{2 \pi} \sigma} e^{-x^{2} / 2 \sigma^{2}} d x
$$

The probability that a value, $x$, drawn from a Gaussian distribution is between two values $a$ and $b$ (i.e. $P(a<x<b))$ is given by,

$$
\int_{a}^{b} \frac{1}{\sqrt{2 \pi} \sigma} e^{-x^{2} / 2 \sigma^{2}} d x=F(b)-F(a)
$$

A variance, $\hat{\sigma^{2}}$, can be estimated from some central portion of the set of some empirical values $x_{i}$, between portions $a$ and $b$ of the distribution, similarly to equation B.1, by the following,

$$
\hat{\sigma^{2}}=\left.\sqrt{\frac{\sum_{i}\left(x_{i}\right)^{2}}{N}}\right|_{a} ^{b}
$$

This formula gives the estimated variance from the central portion of some empirical data. Similarly, the theoretical variance of a Gaussian distribution determined between a restricted integral range $[a, b]$ is given by the following,

$$
\hat{\sigma^{2}}=\frac{1}{F(b)-F(a)} \int_{a}^{b} x^{2} \frac{1}{\sqrt{2 \pi} \sigma} e^{-x^{2} / 2 \sigma^{2}} d x
$$

letting $u=x \Longrightarrow d u=d x$ and letting $v=-\sigma^{2} e^{-u^{2} / 2 \sigma^{2}} \Longrightarrow d v=u e^{-u^{2} / 2 \sigma^{2}} d x$ then equation B. 6 can be solved using integration by parts so that,

$$
\hat{\sigma^{2}}=\frac{1}{F(b)-F(a)}\left[\left.u \frac{1}{\sqrt{2 \pi} \sigma} \sigma^{2} e^{-u^{2} / 2 \sigma^{2}}\right|_{a} ^{b}\right]-\frac{1}{F(b)-F(a)} \int_{a}^{b} \sigma^{2} \frac{1}{\sqrt{2 \pi} \sigma} e^{-u^{2} / 2 \sigma^{2}} d u .
$$

This simplifies down to,

$$
\hat{\sigma^{2}}=\sigma^{2}\left(\frac{1}{F(b)-F(a)}\left[\left.u \frac{1}{\sqrt{2 \pi} \sigma} e^{-u^{2} / 2 \sigma^{2}}\right|_{a} ^{b}\right]+1\right) .
$$

A quantity $q$ is chosen such that for some inner central percentage of the distribution $p$

$$
F(q \sigma)=\int_{\infty}^{p} \frac{1}{\sqrt{2 \pi} \sigma} e^{-x^{2} / 2 \sigma^{2}} d u
$$

and $a$ and $b$ are set to $a=q \sigma$ and $b=q \sigma$. Then equation B. 8 becomes,

$$
\hat{\sigma^{2}}=\sigma^{2}\left(\frac{-2 q}{\tilde{F}(q)-\tilde{F}(-q)} \frac{1}{\sqrt{2 \pi}} e^{-q^{2} / 2}+1\right)
$$


where $\tilde{F}(x)=P(x<\hat{x})$, for a Gaussian probability density function with a variance equal to 1. Setting B.5 equal to B.10 and solving for $\sigma^{2}$, the variance of the distribution estimated from the inner percentage of the distribution $p$ is then given by,

$$
\sigma=\sqrt{1 /\left.\left[\left(\frac{-2 q}{\tilde{F}(q)-\tilde{F}(-q)} \frac{1}{\sqrt{2 \pi}} e^{-q^{2} / 2}+1\right)\right] \frac{\sum_{i}\left(x_{i}\right)^{2}}{N}\right|_{-p} ^{p}} .
$$

i.e. The variance of the distribution is estimated from the variance of the inner central portion of the data given by $\hat{\sigma}^{2}$ is multiplied by the scaling factor $1 /\left[\left(\frac{-2 q}{\tilde{F}(q)-\tilde{F}(-q)} \frac{1}{\sqrt{2 \pi}} e^{-q^{2} / 2}+1\right)\right]$.

\section{B.2.1 Advantages of this method}

There are two key advantages of this method when dealing with real life data.

- If the assumption that there are outliers is false, this method (unlike that of just removing larger values) will not underestimate the standard deviation when a sensible proportion (/inner percentage q) of the data is used to estimate it. This is demonstrated in the figure below, the inner percentage used to estimate the standard deviation of 10,000 randomly selected values from a Gaussian distribution with a zero mean and standard deviation of 5. It can be seen that for inner percentages greater than $20 \%$ the estimated standard deviation is almost exactly 5 .

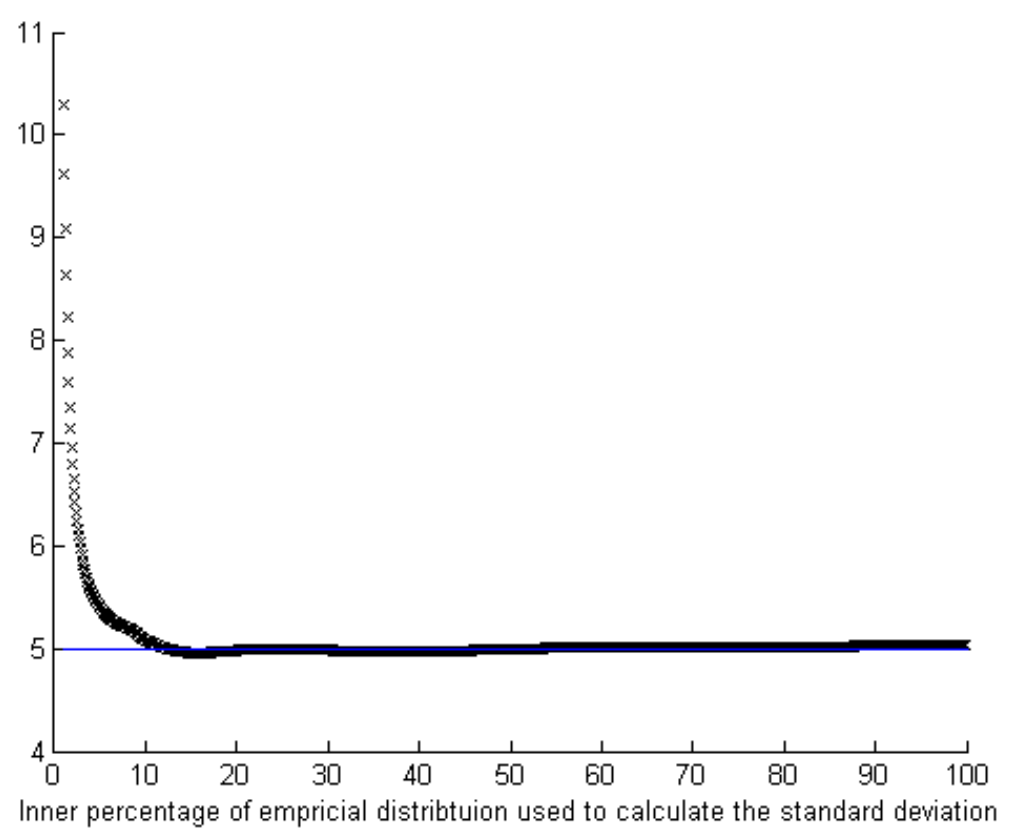

Figure B.2: Standard deviation (y-axis) estimated from inner percentage (x-axis) of Gaussian distribution with a standard deviation of 5 .

- In the presence of outliers, this method provides the best estimate of the majority distribution. For example, consider a dataset which should contain values drawn from a distribution with a standard deviation $\sigma_{x} 5$, consider also that some percentage $p$ of this data set has been contaminated with other data which are drawn from a distribution 
with a larger standard deviation $\sigma_{y}$ e.g. of 20. As the percentage increases, the standard deviation estimated by the standard formula, $\sigma$ of the whole data set increases such that $\sigma=\sqrt{\sigma_{x}^{2} * p+\sigma_{y}^{2}(1-p)}$.

If we need to estimate the standard deviation of the uncontaminated data, estimating the standard deviation using equation B.11 from the inner percentage $q$ will be more representative than considering the whole data set. This is demonstrated in the figure B.3 the standard deviation estimated from the inner $68 \%$ using equation B.11 (black crosses) and the standard deviation of the whole data set as estimated by equation B.1 (blue line) of a mixture of two distributions $X$ and $Y$ where the percentage of value drawn from $Y$ is the x-axis. Where the standard deviation of $X$ is 5 and the standard deviation of $Y$ is 20 . It can be seen the standard deviation estimated by equation B.11 is more representative of the uncontaminated data than the standard method.

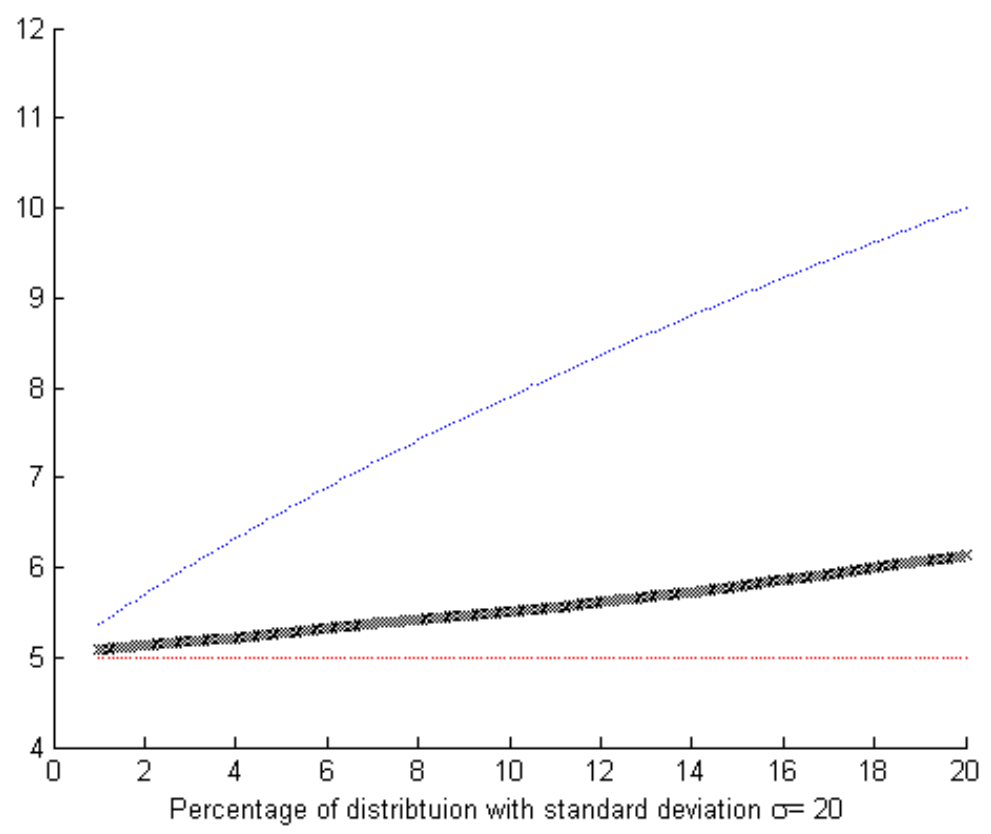

Figure B.3: Standard deviation estimated from inner 68\% (black x's) and the standard deviation of the whole data set (blue points) for a mixture of two Gaussian distributions with standard deviations of 5 and 20 with the percentage of the whole dataset made up of the larger standard deviation distribution shown on the $\mathrm{x}$-axis.

Throughout this research project, when estimating standard deviations, the inner portion of the distribution has been taken as $68 \%$ (which is approximately 1 standard deviation either side of the mean) so that around two thirds of the empirical distribution values $x$ are well modelled by the fitted Gaussian distribution and the estimated standard deviation is not influenced by outliers. 


\section{B.3 A worked example using data from section 4}

Consider the difference between the recorded heights of the terrestrial gravity locations in the GNS Science terrestrial gravity database and those estimated from the $8 \mathrm{~m}$ digital elevation model (discussed in detail in chapter 4), for those with height code 0 . The empirical distribution of the difference is shown in figure B.4, plotted in blue and a Gaussian cumulative distribution function with mean zero and standard deviation, $\sigma$, estimated by equation B.1 is plotted in red.

Empirical cdf plot of height code 0 heights minus DEM heights (blue)

Estimated distribution in red

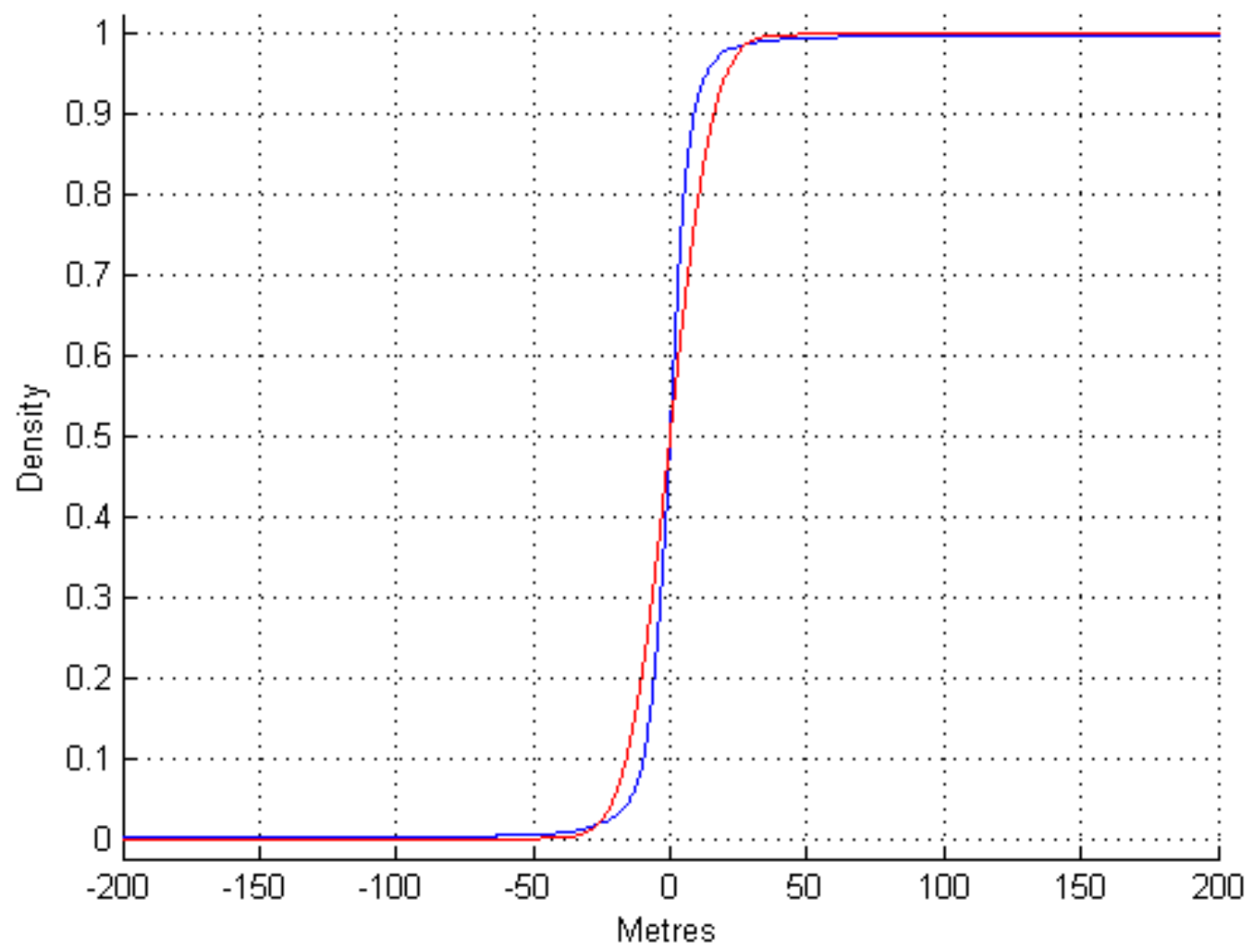

Figure B.4: Empirical cdf plot of height code 0 heights minus DEM heights (blue), Gaussian cumulative distribution function with $\sigma$ estimated by equation B.1 in red.

The standard deviation calculated by equation B.1 here is $12.44 \mathrm{~m}$. This value is clearly far larger than it should be, since the plotted Gaussian distribution does not fit the empirical distribution very well, particularly through the central portion of the distribution. This can be resolved by fitting a Gaussian distribution to just the central portion of the data by equation B.11 .

Figure B.5 shows the empirical distribution of the differences plotted in blue and a Gaussian cumulative distribution function with mean zero and standard deviation estimated from the inner $68 \%$ of the distribution (by the methodology described here) plotted in red.

Here the standard deviation estimated from the inner $68 \%$ of the distribution is $7.58 \mathrm{~m}$. Clearly the fit of the Gaussian distribution to the empirical distribution is better, particularly through 


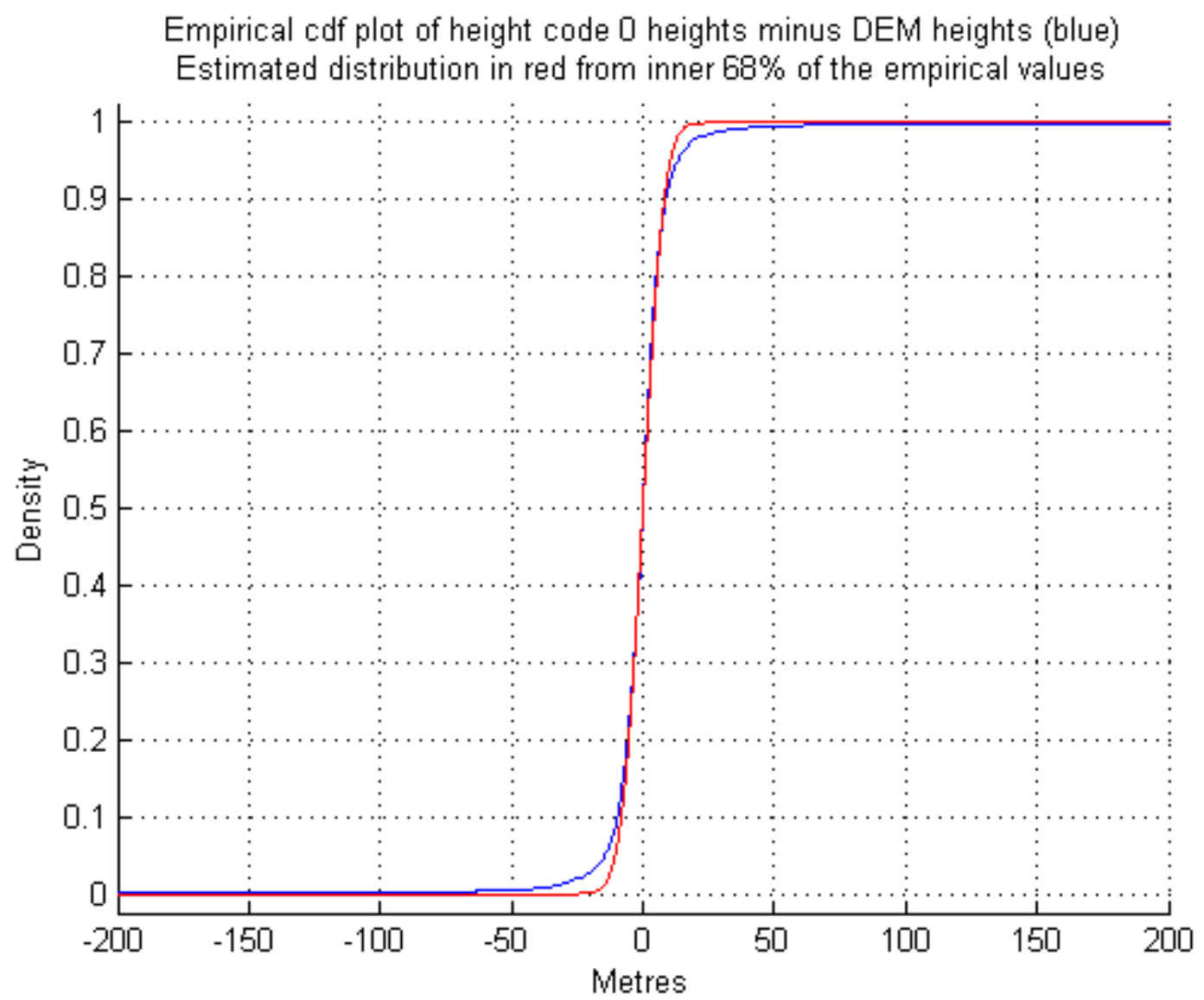

Figure B.5: Empirical cdf plot of height code 0 heights minus DEM heights (blue), Gaussian cumulative distribution function with $\sigma$ estimated from the inner $68 \%$ of the empirical distribution in red.

the central portion, as it is not influenced by outliers. The newly estimated standard deviation is then more representative of the majority of the residuals $x$ than the previous estimate of $12.44 \mathrm{~m}$. 


\section{Appendix C}

\section{Electronic Appendices}

The data and processing scripts used throughout this thesis can be found in the attached electronic appendix. The file tree structure and details of the files inside each directory are listed below.

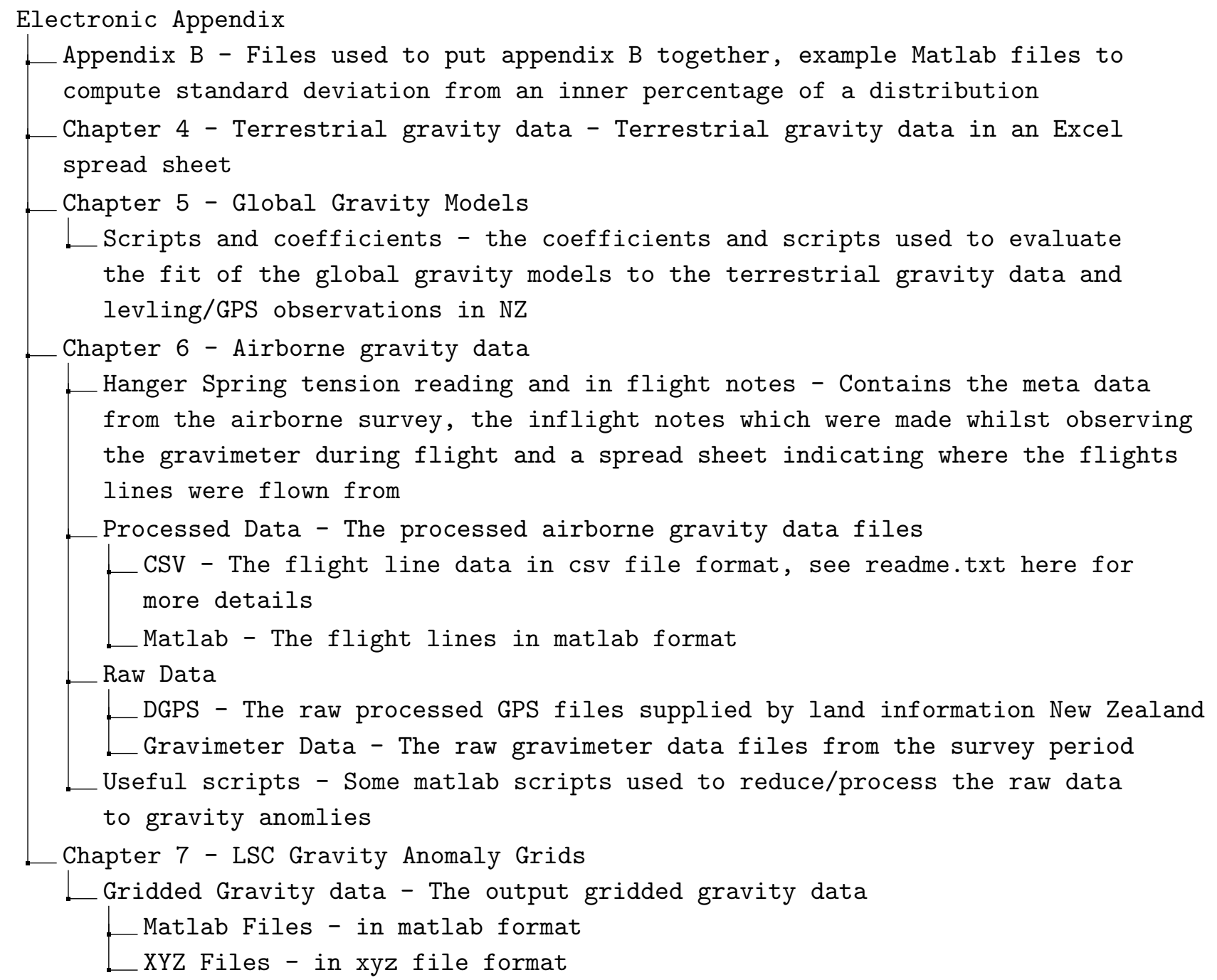


LSC Package - The set of matlab scripts used to perfomr the LSC gridding/data combining

Satellite altimetry - Raw Data - The Sandwell and Smith gravity data

Shipborne gravity data- Raw Data

Chapter 8 - New Quasigeoid

Ligen 6C4 residual gravity anomalies

L Matlab Files - matlab files used to obtain the resdidual gravity grid and comparison to that used for NZGeoid09

LXYZ Files - XYZ files of the residual gravity grid

Quasigeoid

L Matlab - matlab files used to compare the quasigeoid to the leveling/GPS geoid undulations

XYZ - The final quasigeoid grid

Residual Geoid Undulation and Optimal Parameter determination

LFortran scripts and outputs - Fortran files used to obtain the residual geoid undulations for various parameter variations

- Matlab files - Matlab files used to analyse the output residual geoid undulation grids to determine the optimal L and psi parameter combination 


\section{List of Figures}

1.1 An ellipsoid with its two principal parameters - the semi-major (a) and semiminor $(b)$ axes. . . . . . . . . . . . . . . . . . 16

1.2 The separation between points $Q_{0}$ (which is on the reference ellipsoid) and $P$ (on the surface of the Earth). . . . . . . . . . . . . . . . . . 18

1.3 The separation between points $P_{0}$ (which is on the geoid) and $P$ defined as the distance along the plumbline. - from Featherstone and Kuhn 2006 . . . . . . . 20

1.4 The concept of normal heights $H^{N}$ and normal-orthometric heights $H^{N-O}$ - from Featherstone and Kuhn (2006). Plumblines shown here are separated horizontally for illustrative purposes only. . . . . . . . . . . . . . . . . . 22

1.5 Ellipsoidal, orthometric and normal-orthometric heights and the geoid undulation $N$ and height anomaly $\xi$ - adapted from Amos (2007). Again plumblines are separated horizontally for clarity. . . . . . . . . . . . . . . . . 23

2.1 Bouguer slab and residual terrain. . . . . . . . . . . . . . 28

2.2 Left shows the hammer zones radii in $\mathrm{m}$ and number of compartments and Right shows the geometry of the zones. Figure has been adapted from Amos (2007). . . 29

2.3 Terrain correction prism - adapted from Nagy (1966). . . . . . . . . . . . . 30

2.4 An illustration of the geometry for a segment of Hammer's terrain correction for

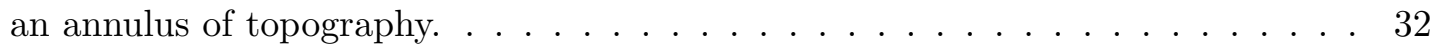

2.5 A comparison of the terrain effect of a $10000 \mathrm{~m}^{2}$ area piece of topography, computed by Nagy prisms (blue) and Hammer's method (red) and difference (green) against the distance from the observation point for 3 elevations 10, 100 and $1000 \mathrm{~m}$ (A,B, and $\mathrm{C}$ respectively) on a $\log _{10}$ scale . . . . . . . . . . . 33

2.6 A map illustrating the specified locations of the 1000 marks chosen at random from the GNS terrestrial gravity database. . . . . . . . . . . . . . . . . 34

2.7 The gravity anomaly caused by a buried sphere with a density anomaly of $0.4 \mathrm{Mg} / \mathrm{m}^{3}$, radius of $200 \mathrm{~m}$ and depths varying from 200 to $500 \mathrm{~m}$. . . . . . . 35

3.1 The 13 local vertical datums which are used in New Zealand. The coloured points indicate the extent of each network. . . . . . . . . . . . . . . 50

3.2 Annual mean sea level estimates from tide gauge data at Auckland, Wellington, Lyttelton and Dunedin. From Hannah and Bell (2012). . . . . . . . . . . . . . . 51

3.3 NZGeoid2009, the quasigeoid used as the reference surface for New Zealand Vertical Datum 2009. . . . . . . . . . . . . . . . . . . . 53

3.4 The processing steps taken in the computation of NZGeoid2009. - adapted from Classens et al. (2009) . . . . . . . . . . . . . . . . . . 55

3.5 Height anomalies derived from the primary precise leveling network and GPS observations. The colour scale is in metres. . . . . . . . . . . . . . 58 
3.6 Figure (a) shows the difference between leveling derived height anomalies and NZGeoid2009 before the bias adjustment. Figure (b) shows the difference between leveling derived height anomalies and NZGeoid2009 after the LVD bias adjustment. Both colour scales are in metres. . . . . . . . . . . . . . . . . 59

3.7 CDF plot of the difference between leveling derived height anomalies and NZGeoid2009 height anomalies before (blue) and after LVD bias adjustment (red), best fitting Gaussian cdf is in black. The LVD bias adjustment improves the standard deviation of the inner $68 \%$ of the distribution by around $8 \mathrm{~cm}$ and also reduces the overall bias of the distribution by $32 \mathrm{~cm}$ in absolute value. . . . . . . 61

4.1 Terrestrial gravity data coverage over the North Island of New Zealand. The density of measurements can be seen to vary accross the island. In areas of scientific interest, such as the Taupo Volcanic Zone, the density increases whereas in rougher terrain, such as in the Opotiki region (in the East) the density reduces. 63

4.2 Terrestrial gravity data coverage over the South Island and Stewart Island of New Zealand. Where the topography is rough the data density reduces, for example through the ranges down the centre of the country or in Fiordland (in the SouthWest) where the data density drops to almost one measurement every $30 \mathrm{~km}$. In flatter regions, the data density is much higher, for example in Canterbury (centre-East). . . . . . . . . . . . . . . . . . .

4.3 Recomputed terrestrial free air anomaly data over the North, South and Stewart Island, gridded at 1 arc minute for illustrative purposes using a linear interpolation algorithm. The scale is in $\mathrm{mGal}\left(10^{-5} \mathrm{~m} / \mathrm{s}^{2}\right)$.

4.4 Terrestrial free air anomaly data over Chatham (top) and Pitt (bottom) Islands, gridded at 1 arc minute for illustrative purposes using a linear interpolation algorithm. The scale is in $\mathrm{mGal}\left(10^{-5} \mathrm{~m} / \mathrm{s}^{2}\right) \ldots \ldots \ldots \ldots$

4.5 Field observation terrain corrections plotted against the inner zone terrain corrections computed by the Hammer prism method. Reference lines are shown at $0.1 \mathrm{mGal}$ and Hammer=Field obs. in blue and $10 \mathrm{mGal}$ in red. The scale is in

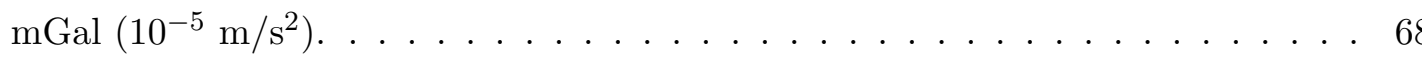

4.6 Terrestrial refined Bouguer anomaly data over the North, South and Stewart Island, gridded at 1 arc minute for illustrative purposes using a linear interpolation

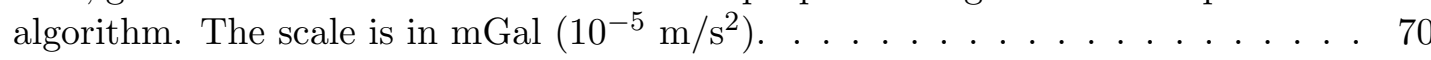

4.7 Terrestrial refined Bouguer anomaly data over Chatham (top) and Pitt (bottom) Islands, gridded at 1 arc minute for illustrative purposes using a linear interpolation algorithm. The scale is in $\mathrm{mGal}\left(10^{-5} \mathrm{~m} / \mathrm{s}^{2}\right) . \ldots \ldots \ldots$. . . . . .

4.8 Empirical CDF of the heights with code 0 minus heights derived from the $8 \mathrm{~m}$ DEM (in blue) and distribution fitted to the inner $68 \%$ (in red). . . . . . . . . 72

4.9 Empirical CDF of the heights with code 1 minus heights derived from the $8 \mathrm{~m}$ DEM (in blue) and distribution fitted to the inner $68 \%$ (in red). . . . . . . . 73

4.10 Empirical CDF of the heights with code 2 minus heights derived from the $8 \mathrm{~m}$ DEM (in blue) and distribution fitted to the inner $68 \%$ (in red). . . . . . . . . . 74

4.11 Derived height standard deviations (in metres) for each gravity observation in the GNS Science terrestrial gravity database. . . . . . . . . . . . . 75

4.12 Linearly gridded estimated error of the terrestrial gravity observations in mGal. . 76

5.1 Error degree variance plots for all satellite only models with GO_CONS_GCF_2_DIR_R5 in red. . . . . . . . . . . . . . . . . . . . . 84 
5.2 Combination scheme with respect to model degree of data sets used to compute EIGEN-6C4. Adapted from slide 4 of Forste et al (2014) . . . . . . . . . . . . 86

6.1 The surveyed flight lines (in white) and calibration lines (in red). The four hangers used during the survey are marked with stars. . . . . . . . . . . . . . . . 91

6.2 Piper Chieftain ZK-RDT . . . . . . . . . . . . . . . . . . 93

6.3 Piper Chieftain ZK-RDT schematic diagram . . . . . . . . . . . . . . . . 93

6.4 Upgraded ZLS LaCoste and Romberg S80 gravimeter used for the New Zealand national airborne gravity survey. . . . . . . . . . . . . . . . . . 95

6.5 Schematic design of the Lacoste and Romber model S gravimeter adapted from Dehlinger (1978) . . . . . . . . . . . . . . . . . . . . 96

6.6 The relative gravity from the gravimeter computed from the raw gravimeter components. The amplitude of the signal is $10^{4} \mathrm{mGal}$, this is significantly larger than what is expected from the gravity field. Corrections must be made to obtain a meaningful gravity signal. . . . . . . . . . . . . . . . . . 98

6.7 The derived absolute vertical accelerations along the flight line measured by the gravimeter. . . . . . . . . . . . . . . . . . . 99

6.8 The GPS derived vertical accelerations along the example flight line. Similar to the relative gravimeter data the amplitude of the GPS derived vertical accelerations is around $10^{4}$ indicating that the aircraft's motion is the likely source of high amplitude signal in the gravimeter measurements. For this reason the GPS derived vertical accelerations must be subtracted from the gravimeter signal. . . 100

6.9 A zoomed in plot of the GPS derived vertical accelerations (red) and de-meaned gravimeter absolute gravity along the example flight line (blue). The two signals are remarkably similar indicating most of what the gravimeter is measuring is the vertical accelerations of the plane. . . . . . . . . . . . . . . . . 101

6.10 The calculated tilt correction along the example flight line. . . . . . . . . . . . 102

6.11 This figure shows the Eotvos correction along the example flight line. The correction is positive since the flight line was flown from South-West to North-East. 103

6.12 The ellipsoidal gravity along the example flight line. There is a negative gradient in the correction since ellipsoidal gravity is strongest at the poles and the flight line was flown from south-west to north-east. . . . . . . . . . . . . . . . . . 104

6.13 The free air correction along the example flight line. . . . . . . . . . . . . . 104

6.14 The noisy free air gravity signal along the example flight line. Note that the signal amplitude is significantly higher than the expected $\pm 200 \mathrm{mGal}$ range of the free air gravity anomaly. The signal is completely masked in high amplitude, high frequency noise. . . . . . . . . . . . . . . . . . 105

6.15 This figure shows the filtered free air gravity signal (in blue) along the example flight line and the EGM2008 gravity anomaly along the same line (red) for comparison. . . . . . . . . . . . . . . . . . . 106

6.16 An illustration of the geometry for a segment of Hammer's terrain correction for an annulus of topography $e-h$ below the observation location. . . . . . . . 107

6.17 This figure demonstrates where zero weights were manually assigned to the erroneous sections of track at the beginning and end of the example flight line. The top plot shows the filtered signal (black), clipped filtered signal (blue) and gravity anomaly from EGM2008 (red) and the bottom plot shows the weights which have been manually assigned to the along track data. . . . . . . . . . . . 109 
6.18 This figure illustrates where zero weights were manually assigned to the data. The gridded data are shown in subplot (a) with the flight line in black. These plots were used to examine the cross track agreement of the data. Subplot (b) shows the raw unfiltered free air gravity signal. Here there is some impulse noise characteristic of the beam reaching the threshold, around $1800 \mathrm{~s}$ and $4700 \mathrm{~s}$. Subplot (c) shows the filter signal (blue) and gravity anomaly from EGM2008 and subplot (d) shows the weights which have been assigned to the data.

6.19 (a) shows all of the 120 second Gaussian filtered free air anomaly data. (b) shows the 120 second Gaussian filtered free air anomaly data with the erroneous data not plotted.

6.20 Along track free air profile showing a bias present in the airborne gravity data along flight line 23. Airborne data is plotted in blue, EGM2008 is plotted in red and the bias adjusted line by the methodology described in the following section is plotted in green. There is a clear dc offset between the red and blue lines, estimated by a mean difference to be $15.7 \mathrm{mGal}$. This bias is not present visually between the red and green lines. . . . . . . . . . . . . . . . . . . 112

6.21 The airborne Bouguer anomaly data for each flight line. Only the data which have not been flagged as erroneous (white gaps) are shown here. . . . . . . . . 116

6.22 (a) shows the $9 \mathrm{~km}$ filtered topography. (b) shows the terrain correction at 5000 m above the reference ellipsoid. . . . . . . . . . . . . . . . . . . . 118

6.23 (a) shows EGM2008 gravity anomaly at $5000 \mathrm{~m}$ above the reference ellipsoid. (b) shows the approximated EGM2008 Bouguer anomaly used for the least squares collocation remove compute restore procedure. . . . . . . . . . . . . . . . . 119

6.24 (a) shows the terrain corrected EGM2008 gravity anomaly along flight lines. (b) Residual gravity anomaly $\Delta \bar{g}_{1}$ along flight lines.

6.25 Empirical covariance is shown in blue and the fitted logarithmic covariance function is shown in green. . . . . . . . . . . . . . . . . . . 121

6.26 Figure (a) shows the estimated biases. The colourbar is in mGal Figure (b) shows the empirical cdf plot of the estimated biases. They have a standard deviation of around 5 mGal. . . . . . . . . . . . . . . . .

6.27 Figure (a) shows the residual gravity signal before the bias adjustment. The colourbar is in mGal. Figure (b) shows the residual gravity signal after the bias

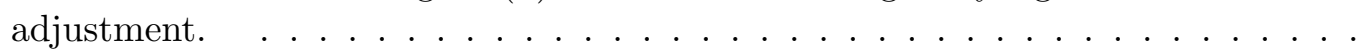

6.28 Figure (a) shows the Bouguer gravity signal before the bias adjustment. The colourbar is in mGal. Figure (b) shows the Bouguer gravity signal after the bias adjustment. . . . . . . . . . . . . . . . . . 126

6.29 Flight lines and tie line intersection points. . . . . . . . . . . . . . 128

6.30 Empirical CDF plot of free air cross-over differences (in blue), Gaussian distribution fitted to the inner $68 \%$ has a standard deviation of $9.3 \mathrm{mGal}$ before bias adjustment and 7.3 mGal after. . . . . . . . . . . . . . . . . . . . 129

6.31 Flight lines 25 (North-East, South-West) and 47 (South-East, North-West) on the South Island and free air anomaly profiles along each line and intersection point marked by a black 'o' . . . . . . . . . . . . . . . . . . . 130

6.32 Flight lines 25 (North-East, South-West) and 47 (South-East, North-West) value at intersection point (top) and the difference (bottom). . . . . . . . . . . 131

6.33 Empirical CDF of EGM2008 cross-over differences (in blue), fitted distribution (in red) with standard deviation of $4.94 \mathrm{mGal}$, with Gaussian filter parameter of 120 seconds. . . . . . . . . . . . . . . . . . . . . 132 
6.34 Left: Flight lines (blue), tie line (red) and intersection points (blue o's), Right: Tie line free air gravity profile in seconds along the line (red) and line values at intersection points (blue o's). . . . . . . . . . . . . . . . . . 133

6.35 Left: Flight lines (blue), tie line (red) and intersection points (blue o's), Right: Tie line Bouguer gravity profile in seconds along the line (red) and line values at intersection points (blue o's). . . . . . . . . . . . . . . . . . 133

6.36 Empirical CDF plot of EGM2008 cross-over differences (in blue), Gaussian distribution fitted to the inner $68 \%$ has a standard deviation of 3.24 mGal. . . . . . 134

6.37 Empirical CDF plot of Bouguer cross-over differences (in blue), Gaussian distribution fitted to the inner $68 \%$ has a standard deviation of $8.4 \mathrm{mGal}$ before bias adjustment and $6.3 \mathrm{mGal}$ after. . . . . . . . . . . . . . . . 136

6.38 Standard deviation of the EGM2008, airborne free air anomaly and bias corrected airborne free air anomaly for varying filter parameters. . . . . . . . . . . . 137

6.39 Standard deviation of the EGM2008, airborne Bouguer anomaly and bias corrected airborne Bouguer anomaly for varying filter parameters. . . . . . . . . . 137

6.40 Standard deviation of the estimated noise level and cross-over difference standard deviation for EGM2008. The two lines intersect at around 120 seconds, this indicates that more gravity signal is being filtered than noise beyond 120 seconds. 138

6.41 Empirical CDF plot of cross-over differences of least squares collocation downward continued airborne Bouguer gravity anomaly to the cross tie measurement locations. Before bias adjustment is plotted in blue and after the bias adjustment in red. Gaussian distributions are fitted to the inner $68 \%$ of each empirical distribution and are plotted in black. The standard deviation of the (inner $68 \%$ of) cross-over differences before the bias adjustment is $7.9 \mathrm{mGal}$ and after the bias adjustment is 5.9 mGal. . . . . . . . . . . . . . . . . . . . . . . . 140

6.42 Figure (a) shows the gravity anomaly at the height of the topography in mGal. Figure (b) shows terrain corrected gravity anomaly in mGal. This signal was subtracted from the airborne Bouguer gravity anomalies to obtain a zero mean residual gravity anomaly. . . . . . . . . . . . . . . . . . . . . 142

6.43 Figure (a) shows the Terrestrial minus the airborne Bouguer gravity anomalies before the bias adjustment. The colourbar is in mGal Figure (b) shows Terrestrial minus the airborne Bouguer gravity anomalies. The large difference to the East in the Central south Island can be attributed to airborne data loss in this region and can be seen in figure 6.29 , and is resolved when combining the data sets in section 7.1

6.44 Empirical cdf plots of the terrestrial Bouguer anomaly data minus the downward continued airborne data before (black) and after the bias correction (red). Gaussian distributions fitted to the inner $68 \%$ of the distributions are seen as dashes. . . . . . . . . . . . . . . . . . . . . . . . . . 144

6.45 The free air anomaly of 5 separate flights, corrected for flight line biases, along the North Island calibration line and an average in green. The standard deviation from the average is $2.21 \mathrm{mGal}$.

6.46 The free air anomaly of 5 separate flights along the South Island calibration line and an average in green. The standard deviation from the average is $2.53 \mathrm{mGal}$.

6.47 The airborne Bouguer anomaly downward continued to the topographic surface along the North Island calibration line in red and terrestrial Bouguer anomaly in blue. . . . . . . . . . . . . . . . . . . . . . . . . 147 
6.48 The airborne Bouguer anomaly downward continued to the topographic surface along the South Island calibration line in red and terrestrial Bouguer anomaly in blue. . . . . . . . . . . . . . . . . . . . . . . . . 148

7.1 1 arc minute grid of topographic heights from block averaged $8 \mathrm{~m}$ DEM heights. This figure shows the full computation area although in other figures throughout this section the area has been restricted for illustrative purposes. . . . . . . . . 155

7.2 Least squares collocation gridded terrestrial gravity observations in mGal. . . . . 156

7.3 Least squares collocation gridded propagated terrestrial gravity observations error in mGal. . . . . . . . . . . . . . . . . . . . . . . . . . . 157

7.4 Least squares collocation gridded terrestrial gravity observations in mGal in the Taupo volcanic zone (a) and Otago region (b). There are undesirable "bulls eye" artifacts in the gridded data. The black marks indicate the measurement locations.158

7.5 Least squares collocation gridded terrestrial gravity observations error in mGal in the Taupo volcanic zone (a) and Otago region (b) . . . . . . . . . . . 158

7.6 (a) Least squares collocation gridded terrestrial gravity observations in mGal. Minimum noise increased to $1 \mathrm{mGal}$. (b) Least squares collocation gridded propagated terrestrial gravity observations error in mGal. Minimum noise increased

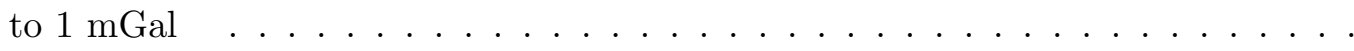

7.7 Least squares collocation gridded terrestrial gravity observations in $\mathrm{mGal}$ in the Taupo volcanic zone (a) and Otago region (b). The undesirable "bulls eye" artifacts in the gridded data have been removed by increasing the minimum noise level to 1 mGal. . . . . . . . . . . . . . . . . . . . 160

7.8 Gravity anomaly difference maps for the two regions. (New minus old) . . . . . 160

7.9 (a) Difference maps between least squares collocation gridded terrestrial gravity observations with a minimum noise increased to $1 \mathrm{mGal}$ and the original noise values. (b) Difference maps between least squares collocation gridded terrestrial gravity observations propagated error with a minimum noise increased to $1 \mathrm{mGal}$ and the original noise values . . . . . . . . . . . . . . . 162

7.10 Covariance of Gaussian filtered noise with $\sigma$ parameter 120 with time lag t. . . . 164

7.11 (a) shows the least squares collocation gridded airborne gravity observations in mGal with just diagonal noise elements, (b) shows least squares collocation gridded airborne gravity observations propagated error in $\mathrm{mGal}$ with just diagonal noise elements. . . . . . . . . . . . . . . . . . . . 165

7.12 (a) shows the least squares collocation gridded airborne gravity observations in mGal with off diagonal noise matrix elements, (b) shows the least squares collocation gridded airborne gravity observations propagated error in mGal with off diagonal noise matrix elements. . . . . . . . . . . . . . . . . 166

7.13 (a) shows the least squares collocation gridded airborne gravity observations in $\mathrm{mGal}$ with just diagonal noise elements minus the grid with off diagonal noise elements, (b) shows the least squares collocation gridded airborne gravity observations propagated error in $\mathrm{mGal}$ with off diagonal noise elements minus the grid with off diagonal noise elements. . . . . . . . . . . . . . . .

7.14 Least squares collocation gridded airborne gravity observations in mGal around the Taranaki Bight using just diagonal noise matrix elements (a) and off diagonal noise matrix elements (b) . . . . . . . . . . . . . . . . . . . 168

7.15 (a) shows the least squares collocation combined airborne and terrestrial gravity observations in mGal, (b) shows the propagated error in mGal. . . . . . . . . . 169 
7.16 Ship track data coverage in the region 160 (E) to $190(\mathrm{E})$ and $25(\mathrm{~S})$ to $60(\mathrm{~S})$. Adapted from Amos (2007) appendix C. . . . . . . . . . . . . . 171

7.171 arc minute gridded Sandwell et al (2014) gravity anomaly in mGal. . . . . . . . 173

7.181 arc minute gridded Sandwell et al (2014) gravity anomaly error in mGal. . . . 174

7.191 arc minute gridded Sandwell et al (2014) gravity anomaly error in mGal masked onshore and near offshore. . . . . . . . . . . . . . . . . . . . . 175

7.201 arc minute gridded Sandwell et al (2014) gravity anomaly masked with indicative marks (in black) where the other data are. There is good data coverage, from the terrestrial, airborne and shipborne data, where the satellite data have been removed and in transitional areas. . . . . . . . . . . . . . . . . 176

7.21 Topography corrected gravity anomaly map in the region 160 (E) to 190 (E) and $25(\mathrm{~S})$ to $60(\mathrm{~S})$ gridded using least squares collocation using the airborne, terrestrial, shipborne and satellite gravity data. The scale is in mGal. . . . . . . 177

7.22 Gravity anomaly propagated error map in the region 160 (E) to 190 (E) and 25 (S) to 60(S) gridded using least squares collocation using the airborne, terrestrial, shipborne and satellite gravity data. The scale is in mGal. . . . . . . . . . . . . . 178

8.1 Figure (a) shows the new gridded Faye anomaly using the terrestrial, airborne, satellite altimetry and shipborne gravity data and 1 arc minute topographic heights. Figure (b) shows the Faye anomaly map used to calculate NZGeoid2009. Both colour scales are in mGal. . . . . . . . . . . . . . . . . . . . . . . . 182

8.2 Figure (a) shows the new gridded Faye anomaly using the terrestrial, airborne, satellite altimetry and shipborne gravity data and 1 arc minute topographic heights minus the Faye anomaly map used to calculate NZGeoid2009. Mostly the features are high frequency. Figure b) shows the same difference map zoomed in and demonstrates the large differences around coastal areas. Both colour scales are in mGal. . . . . . . . . . . . . . . . . . . . . . . . . . . . . . 183

8.3 Figure (a) shows the Eigen-6C4 gravity anomaly gridded at the locations of the gridded Faye anomaly. Figure (b) shows the gridded Faye anomaly minus the Eigen-6C4 gravity anomaly. The scales are in mGal. . . . . . . . . . . . . . . . 186

8.4 Figure (a) shows the Eigen-6C4 quasigeoid height anomalies gridded at the locations of the gridded Faye anomaly. Figure (b) shows the primary indirect topographical effect. The scales are in metres. . . . . . . . . . . . . . . 187

8.5 Standard deviation estimated from the inner $68 \%$ of the leveling height anomalies minus the quasigeoid computed with parameters $\psi_{0}$ and $L$. . . . . . . . . . . 189

8.6 Root mean squared of the leveling height anomalies minus the quasigeoid computed with parameters $\psi_{0}$ and $L \ldots \ldots$. . . . . . . . . . . . . . 189

8.7 Figure (a) shows the residual quasigeoid for parameter choices $\psi_{0}=1.5$ and $L=280$. Figure (b) shows the quasigeoid after restoring the global gravity model and topographic effect. The scales are in cm. . . . . . . . . . . . . . . 191

8.8 Figure (a) shows the quasigeoid heights computed with parameters $\psi_{0}=1.5$ and $L=280$ minus the leveling derived height anomalies. Figure (b) shows the NZGeoid2009 quasigeoid heights minus the leveling derived height anomalies . The scales are in cm. . . . . . . . . . . . . . . . . . . . . . . . 192

8.9 Standard deviation of the inner $68 \%$ of the leveling height anomalies minus the quasigeoid computed with parameters $\psi_{0}$ and $L$. Scale is in $\mathrm{cm}$. . . . . . . . 195

8.10 Root mean squared of the leveling height anomalies minus the quasigeoid computed with parameters $\psi_{0}$ and $L$. Scale is in $\mathrm{cm} \ldots \ldots$. . . . . . . . 195 
8.11 Figure (a) shows the quasigeoid heights computed with parameters $\psi_{0}=1.5$ and $L=280$ minus all the leveling derived height anomalies. Figure (b) shows the NZGeoid2009 quasigeoid heights minus all the leveling derived height anomalies . The scales are in metres. . . . . . . . . . . . . . . . . . . . . . 196

A.1 Graphical representation of the change in the strength of gravity due to a tilt. Adapted from Model 'S' Air Sea Dynamic Gravity Meter System II, Instruction Manual (2006) . . . . . . . . . . . . . . . . . . . . . . . . . . 214

A.2 The absolute value of the LACC accelerometer recording and derived value $A$ for various tilt angles a line of best fit with gradient $k=26.4830 \ldots . . . . . .216$

A.3 The absolute value of the XACC accelerometer recording and derived value $A$ for various tilt angles a line of best fit with gradient $k=25.1101 \ldots$. . . . . . 216

B.1 Standard deviation (y-axis) calculated from inner percentage (x-axis) of Gaussian

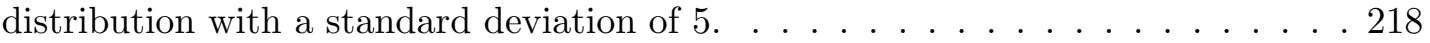

B.2 Standard deviation (y-axis) estimated from inner percentage (x-axis) of Gaussian distribution with a standard deviation of $5 . \ldots 220$

B.3 Standard deviation estimated from inner $68 \%$ (black x's) and the standard deviation of the whole data set (blue points) for a mixture of two Gaussian distributions with standard deviations of 5 and 20 with the percentage of the whole dataset made up of the larger standard deviation distribution shown on the x-axis. . . . 221

B.4 Empirical cdf plot of height code 0 heights minus DEM heights (blue), Gaussian cumulative distribution function with $\sigma$ estimated by equation B.1 in red. . . . . 222

B.5 Empirical cdf plot of height code 0 heights minus DEM heights (blue), Gaussian cumulative distribution function with $\sigma$ estimated from the inner $68 \%$ of the empirical distribution in red. 


\section{List of Tables}

5.1 Satellite only global gravity models compared. . . . . . . . . . . . . . . 80

5.2 Combined global gravity models compared . . . . . . . . . . . . . . . 81

5.3 Table of statistics of the terrestrial gravity free air anomalies at 40667 locations minus $\Delta g$ computed from the 37 satellite only global gravity models in mGals. $\quad 82$

5.4 Table of statistics of the leveling derived height anomalies at 1442 locations minus $\xi$ computed from the 37 satellite only global gravity models. The residual for each of the 13 local vertical datums has been demeaned for each model as discussed in section 3.4 i.e. to remove any local vertical datum offsets. Values are in metres. 83

5.5 Table of statistics of the terrestrial gravity free air anomalies at 40667 locations minus $\Delta g$ computed from the 9 combined global gravity models in mGals. . . . . 85

5.6 Table of statistics of the leveling derived height anomalies at 1442 locations minus $\xi$ computed from the 9 combined global gravity models. The residual for each of the 13 local vertical datums has been demeaned for each model as discussed in section 3.4 i.e. to remove any local vertical datum offsets. Values are in metres. . 85

6.1 The filter sigma parameter in seconds and free air cross-over difference standard deviation (of the inner 68\% following appendix B) before and after bias correction.129

6.2 Table of filter parameters and EGM2008 synthetic flight line cross-over difference standard deviations in mGal. . . . . . . . . . . . . . . . . . . . 132

6.3 Table of filter parameters and terrain corrected EGM2008 synthetic flight line cross-over difference standard deviations in mGal. . . . . . . . . . . . . . . . . . 134

6.4 The filter sigma parameter in seconds and Bouguer cross-over difference standard deviation (estimated from the inner 68\%) before and after bias correction. . . . . 135

6.5 Statistics of the Terrestrial Bouguer anomaly minus the downward continued airborne gravity data before and after the bias correction. The airborne data after the bias adjustment fit the terrestrial gravity data better. . . . . . . . . . . 144

6.6 Statistics of the terrestrial Bouguer gravity anomalies minus the downward continued Bouguer gravity anomalies for the North and South Island calibration lines. . . . . . . . . . . . . . . . . . . . . . . . . . 148

7.1 Commonly used covariance functions for geophysical mapping - adapted from Neda (2009). . . . . . . . . . . . . . . . . . . . . . . 153

7.2 The cross-over statistics for the shipborne gravity data prior to the cross-over adjustment - adapted from Amos (2007) appendix C. . . . . . . . . . . . 170

7.3 The cross-over statistics for the shipborne gravity data after the cross-over adjustment - adapted from Amos (2007) appendix C . . . . . . . . . . . . 170

8.1 Standard deviation estimated from the inner $68 \%$ of the distribution of leveling derived height anomalies minus the quasigeoid height anomalies derived with parameter combinations $L$ and $\psi_{0}$. The units are centimetres. . . . . . . . . . 188 
8.2 Root mean squared of the distribution of leveling derived height anomalies minus the quasigeoid height anomalies derived with parameter combinations $L$ and $\psi_{0}$. The units are centimetres. . . . . . . . . . . . . . . . . . . . 188

8.3 Statistics of the difference between leveling derived height anomalies, and those of the $\psi_{0}=1.5$ and $L=280$ quasigeoid, NZGeoid2009 and Eigen-6C4. Values are in cm. . . . . . . . . . . . . . . . . . . . . . . 190

8.4 Standard deviation of the inner $68 \%$ of the differences between the leveling derived height anomalies and the $\left(\psi_{0}=1.5, L=280\right)$ quasigeoid, and NZGeoid2009 193

8.5 Root mean squared of the differences between the leveling derived height anomalies and the $\left(\psi_{0}=1.5, L=280\right)$ quasigeoid, and NZGeoid2009 . . . . . . . 193

8.6 Local vertical datum offsets given for NZGeoid2009 and computed for the original leveling data. Values are given in cm. . . . . . . . . . . . . . . . . . 194

8.7 Std. dev. of inner $68 \%$ of 2857 leveling height anomalies minus quasigeoid for $\psi_{0}$

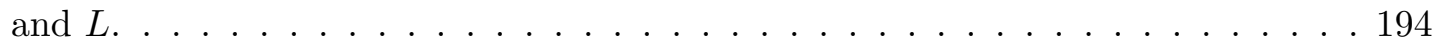

8.8 RMS of 2857 leveling height anomalies minus quasigeoid for $\psi_{0}$ and $L \ldots \ldots$. . . 194

A.1 Results of tilting the gravimeter through an axis parallel to the XACC accelerometer to record changes in the LACC accelerometer. . . . . . . . . . . . . . 215

A.2 Results of tilting the gravimeter through an axis parallel to the LACC accelerometer to record changes in the XACC accelerometer. . . . . . . . . . . . . 215 